\title{
Contaminant trends in reservoir sediment cores as records of influent \\ stream quality
}

Peter C. Van Metre* and Barbara J. Mahler

No. ES $049859 x$

No. of figures: 5

No. of pages of text: 5

No. of tables: 14

\section{Contents}

\section{Description of age dating of cores}

\section{Data Tables}

Table A1. Radionuclide concentrations and assigned deposition dates for sediment cores.

Table A2. Suspended sediment concentrations and selected grain size distributions in suspended sediment. Table A3. Selected major and trace element concentrations in bottom and suspended sediments.

Table A4. Selected chlorinated hydrocarbon compound concentrations in bottom and suspended sediments. Table A5. Selected polycyclic aromatic hydrocarbon compound concentrations in bottom and suspended sediment samples.

\section{Quality Assurance Data Tables}

Table B1. Organochlorine compound quality control samples.

Table B2. Chlorinated hydrocarbon duplicate samples.

Table B3. Polycyclic aromatic hydrocarbon quality control samples.

Table B4. Polycyclic aromatic hydrocarbon duplicate samples.

Table B5. Sample groups for organics analyses and duplicate sample relative percent differences.

Table B6. Major and trace element quality control samples.

Table B7. Sample groups for major and trace elements and forms of carbon analyses and duplicate and triplicate sample relative percent differences.

Table B8. Major and trace element duplicate and triplicate samples.

Table B9. Quality control sample results included with samples analyzed for ${ }^{137} \mathrm{Cs}$. 


\section{Description of age dating of cores}

By Peter C. Van Metre and Barbara J. Mahler

Details of age dating of the cores are presented below for each of the five lakes. The presentations follow a consistent format where the basis and corroboration are presented. Basis is the date markers, approach, and other information in the core that were used to calculate age dates. Corroboration is any date markers and other information in the core or watershed history that support the age assignments made.

\section{Lake Como, Tex.}

Lake Como is a small reservoir constructed in 1889 (Vicki Stokes, City of Fort Worth, oral communication) in west-central Fort Worth, Tex. The reservoir is in a low-income residential area and near interstate highway 30. Three locations were sampled in March 2001 in the lower (core CMO.1), middle (core CMO.3), and upper (core CMO.5) parts of the reservoir. Age dating for CMO.1 is presented here; dates were estimated for the other two cores by Van Metre and others (2003).

\section{Lower lake}

CMO.1 Gravity core $96 \mathrm{~cm}$ long analyzed for all constituents.

Basis The bottom of CMO.1 penetrated a stiff, tan clay at $95 \mathrm{~cm}$, although no root hairs or sand were found, common indicators of pre-reservoir soils. The ${ }^{137} \mathrm{Cs}$ profile has a peak at 80 to $85 \mathrm{~cm}$ (Figure 1). The peak is sharp and activities decrease smoothly to the top of the core. The peak to top of core ratio of 10 , even without decay correcting the older peak sample, indicates the lack of post-depositional mixing in the core. The presence of ${ }^{137} \mathrm{Cs}$ at the bottom of the core and the location of the peak activity indicate that this core only penetrated to the late-1950s. A mass accumulation rate (MAR) of $1.54 \mathrm{~g} / \mathrm{cm}^{2} \mathrm{yr}$ was calculated using the ${ }^{137} \mathrm{Cs}$ peak and the sampling date at the top of the core. Age dates were assigned using this MAR. Corroboration Age assignments are generally corroborated by a very large peak in lead that was dated as 1969.1 (Figure 1) and maximum PCB and total DDT concentrations in the bottom sample analyzed, dated as 1958.6.
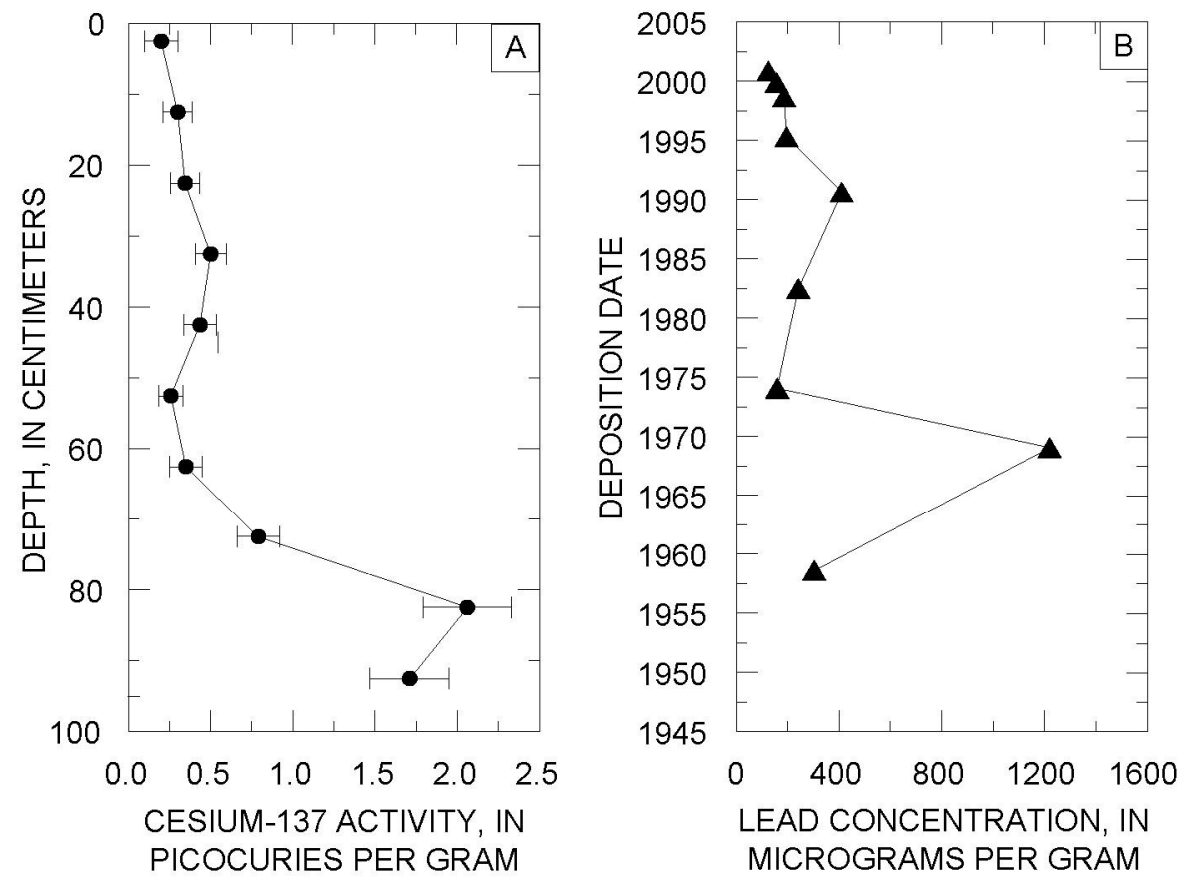

Figure 1. Chemical constituent profiles used to estimate age dates for Lake Como, Tex.

Echo Lake, Tex. 
Echo Lake is a small reservoir constructed in 1919 (Vicki Stokes, City of Fort Worth, oral communication) in south-central Fort Worth, Tex. The reservoir is in a low-income residential and industrial area and near interstate highway 35 west. Three locations were sampled in March 2001 in the lower (core ECO.1), middle (core ECO.4), and upper (core ECO.3) parts of this small reservoir. Only age dating for ECO.1 is presented here; however, dates were assigned to the other two cores by Van Metre and others (2003).

\section{Lower lake}

ECO.1 Gravity core $97 \mathrm{~cm}$ long analyzed for all constituents.

Basis None of the cores penetrated to the pre-reservoir land surface. Core ECO.1 was $97 \mathrm{~cm} \mathrm{long,}$ compared to $80 \mathrm{~cm}$ at the mid-lake site (core ECO.4) and $55 \mathrm{~cm}$ at the upper lake site (core ECO.3). The ${ }^{137} \mathrm{Cs}$ activity profile for ECO.1 contains a pronounced peak at 70 to $75 \mathrm{~cm}$ (Figure 2). The peak is sharp and decreases smoothly to the top of the core, indicating continuous sediment deposition at the site. The peak to top of core ratio of about 20 indicates no significant post-depositional mixing in this core. The presence of ${ }^{137} \mathrm{Cs}$ at the bottom of the core and the location of the peak activity indicate that this core only penetrated to the early-1950s. An MAR of $0.91 \mathrm{~g} / \mathrm{cm}^{2} \mathrm{yr}$ was calculated using the ${ }^{137} \mathrm{Cs}$ peak at $72.5 \mathrm{~cm}$ and the sampling date at the top of the core. Age dates were assigned using this MAR.

Corroboration Age assignments are corroborated by peaks in lead and DDE that are dated as 1972.5 (fig. 2). The deepest sample received an estimated deposition date of 1951.4.

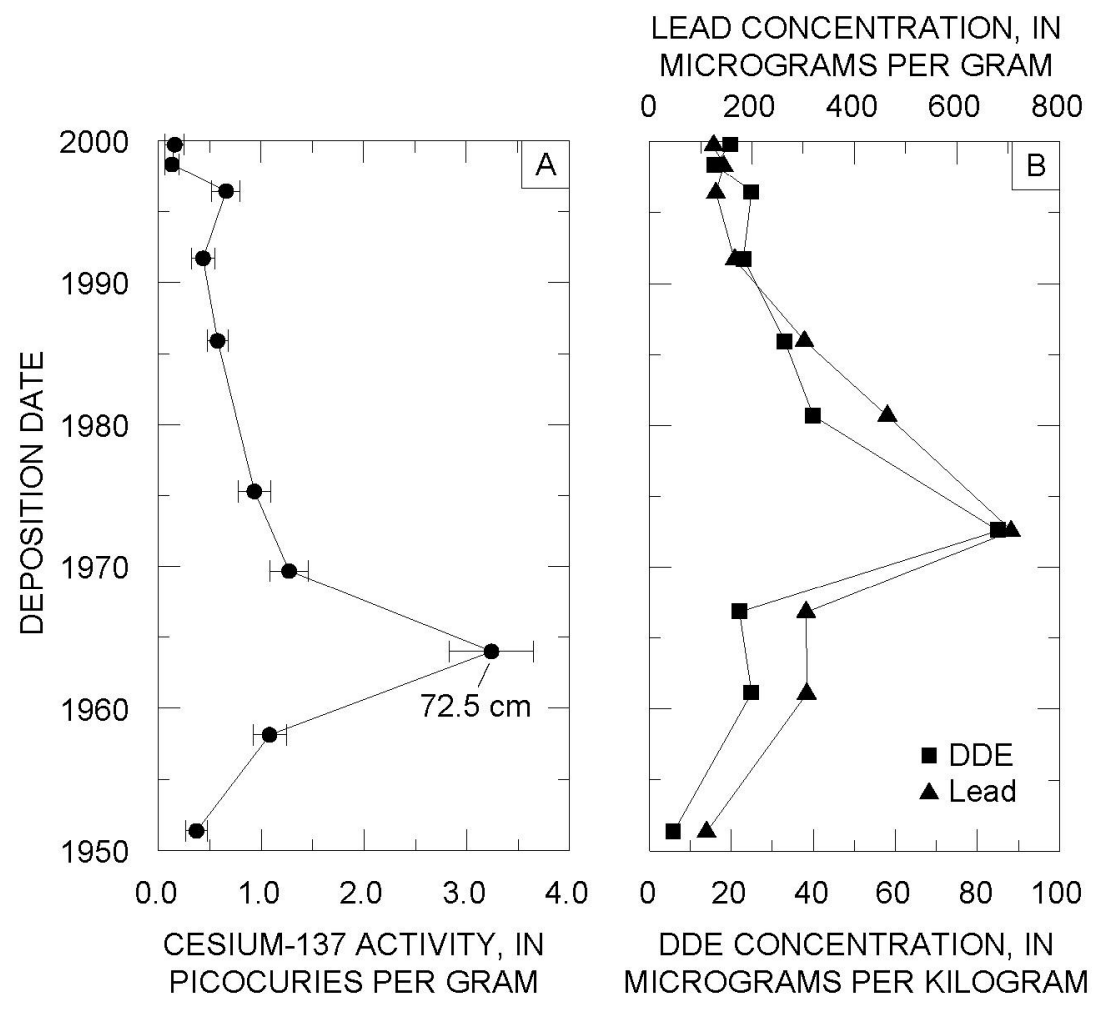

Figure 2. Chemical constituent profiles used to estimate age dates for Echo Lake, Tex.

\section{Fosdic Lake, Tex.}

Fosdic Lake is a small reservoir constructed between 1909 and 1912 (Vicki Stokes, City of Fort Worth, oral communication) in east-central Fort Worth, Tex. The reservoir is in a middle-class residential area near interstate highway 30. Three locations were sampled in March 2001 in the lower (core FOS.2), middle 
(core FOS.4), and upper (core FOS.5) parts of the reservoir. Only age dating for FOS.4 is presented here; however, dates were assigned to the other two cores by Van Metre and others (2003).

Mid-lake

FOS.4 Gravity core $108 \mathrm{~cm}$ long analyzed for all constituents.

Basis The core penetrated the pre-reservoir land surface at $105 \mathrm{~cm}$. A construction date of 1910 was used in age dating the core. The ${ }^{137} \mathrm{Cs}$ activity profile has a pronounced peak at 50 to $55 \mathrm{~cm}$ and supports the assumption that the sediments represent the whole period from 1910 to 2001 (Figure 3 ). Using the ${ }^{137} \mathrm{Cs}$ activity peak as a date marker of 1964.0, the pre-reservoir land surface as 1910, and the sampling date at the top of the core, MARs of 0.69 and $0.57 \mathrm{~g} / \mathrm{cm}^{2}$ yr were calculated for 1910 to 1964 and 1964 to 2001 , respectively. Age dates were assigned to the core using these MARs.

Corroboration Age assignments are generally corroborated by a large peak in lead that was dated as 1968.9 (Figure 3), maximum PCB and total-DDT concentrations in 1968.9, and the first occurrence of organochlorine compounds in the mid-1940s.
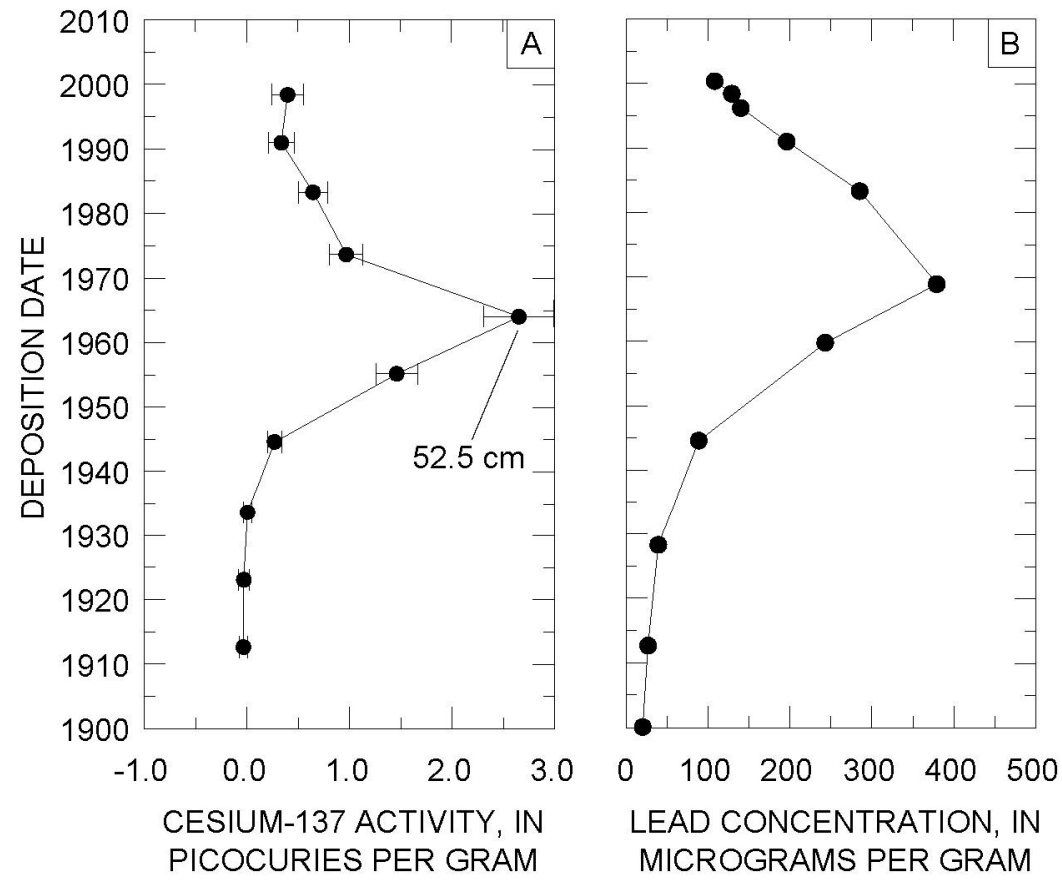

Figure 3. Chemical constituent profiles used to estimate age dates for Fosdic Lake, Tex.

\section{Harris Pond, Mass.}

Harris Pond is a small reservoir located in southeastern Massachusetts north of the city of Woonsocket, R.I. Harris Pond is a supplemental water source for the city of Woonsocket, R.I. The rservoir was originally dammed in the 1880 s, destroyed by a hurricane in 1954, and reconstructed in 1968 (personal communication, Woonsocket Water Department). Harris Pond probably did not drain completely when the original dam was destroyed because many areas of the reservoir are poorly connected and flow ponded behind a railroad causway construced across the middle of the lake (personal communication, Ann Chalmers, U.S. Geological Survey). Three locations were sampled for chemical analysis in July 2000 in the lower (core HSP.1), middle (core HSP.B2), and upper (core HSP.B3) parts of the reservoir.

Location HSP.1

HSP.1 One push core $42 \mathrm{~cm}$ long analyzed for all constituents. 
Basis The peak ${ }^{137} \mathrm{Cs}$ activity from 21 to $24 \mathrm{~cm}$ was assigned a date of 1964.0 (Figure 4A). The average MAR for the 1964 to 2000 interval of the core of $0.18 \mathrm{~g} / \mathrm{cm}^{2} \mathrm{yr}$ was used to assign dates to the core.

Corroboration The lead profile has a sharp peak that was dated as 1968.8 (Figure 4B). Sample intervals are relatively wide in this core, with this sample covering estimated deposition from about 1966 to 1972.

${ }^{137} \mathrm{Cs}$ was detected in the deepest lacustrine sample at 24 to $27 \mathrm{~cm}$, dated as 1955.6 and at the pre-reservoir surface $(25 \mathrm{~cm})$.

\section{Location HSP.B2}

HSP.B2 One box core $29 \mathrm{~cm}$ deep analyzed for all constituents.

Basis The peak ${ }^{137} \mathrm{Cs}$ activity from 23 to $24 \mathrm{~cm}$ was assigned a date of 1964.0 (Figure 4A). The average MAR for the 1964 to 2000 interval of the core of $0.17 \mathrm{~g} / \mathrm{cm}^{2} \mathrm{yr}$ was used to assign dates to the core.

Corroboration The lead profile has a broad high extending from the late-1950s to about 1972 (Figure 4B). The decrease in the early 1970 s is consistent with lead trends elsewhere. ${ }^{137} \mathrm{Cs}$ was detected to the deepest lacustrine sample at 27 to $28 \mathrm{~cm}$ and was assigned a reasonable date of 1953.4.

\section{Location HSP.B3}

HSP.B3 One box core $30 \mathrm{~cm}$ deep analyzed for all constituents.

Basis The ${ }^{137} \mathrm{Cs}$ activity profile, with no peak, indicates that this core does not date back to before 1964 . Lack of a clear date marker led to a decision not to assign dates to this core.
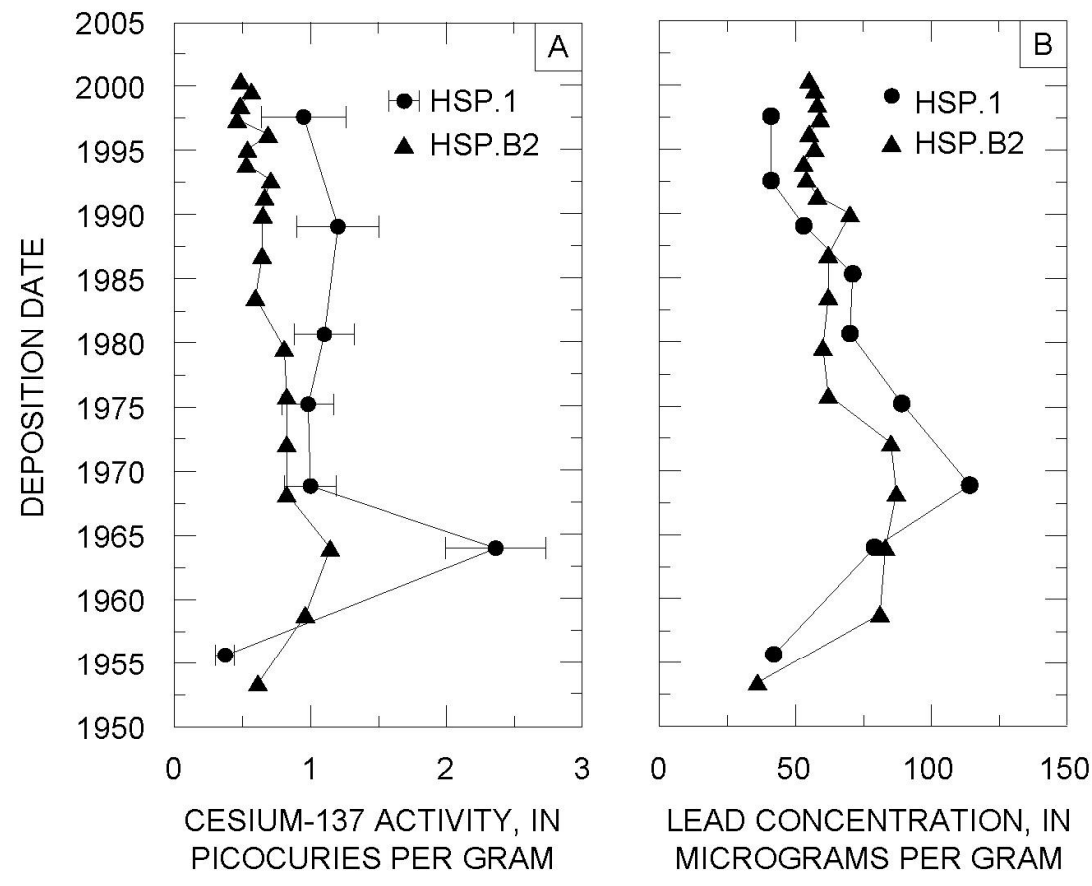

Figure 4. Chemical constituent profiles used to estimate age dates for Harris Pond, Mass.

\section{Upper Mystic Lake, Mass.}

Upper Mystic Lake is located northwest of Boston, Mass., in the town of Winchester. The Aberjona River flows into Upper Mystic Lake and the Mystic River flows out of Lower Mystic Lake, which is immediately downsteam of Upper Mystic Lake. Two locations in the northernmost lobe of the lake were sampled in August 2000 - a box core was collected near the center and a gravity core was collected in the southern end of the lobe.

\section{Location MYS.B2}

MYS.B2 One box core 45-cm deep analyzed for all constituents. 
Basis Two dating approaches were used for this core resulting in reasonable dates for the upper part of the core but conflicting results and uncertainty for the deeper part of the core. Using the ${ }^{137} \mathrm{Cs}$ peak at 26 to 28 $\mathrm{cm}$ as a marker of 1964.0 results in an MAR of $0.16 \mathrm{~g} / \mathrm{cm}^{2} \mathrm{yr}$ (Figure 5A). This MAR yields dates that are reasonably corroborated by the peak in total lead (1970-75), peaks in total DDT and PCBs (1967) and the first occurrence of chlordane, DDTs and PCBs (1950). However, these dates are not in agreement with dates estimated using ${ }^{210} \mathrm{~Pb}$, especially in the lower part of the core (below about $30 \mathrm{~cm}$ ). Several approaches were tried using ${ }^{210} \mathrm{~Pb}$. The unsupported ${ }^{210} \mathrm{~Pb}$ profile has three breaks in slope, one at about $8 \mathrm{~cm}$ indicating a mixing zone to that depth, one at about $22 \mathrm{~cm}$, and a third below $36 \mathrm{~cm}$, defined by only two samples, suggesting a much lower MAR at the bottom of the core $(36$ to $40 \mathrm{~cm}$ ) (Figure 5A). The CRS model (Appleby and Oldfield, 1992) and applied assuming the whole unsupported ${ }^{210} \mathrm{~Pb}$ inventory was captured by the core resulted in a date of 1930 for the ${ }^{137} \mathrm{Cs}$ peak, an indication that the ${ }^{210} \mathrm{~Pb}$ inventory is not complete. Somewhat better results were achieved using the CF:CS model, assuming the top $8 \mathrm{~cm}$ of the core was instantaneously mixed, and fitting two regression lines ( 8 to $22 \mathrm{~cm}, \mathrm{r}^{2}=0.974$ and 20 to $36 \mathrm{~cm}, \mathrm{r}^{2}$ $=0.987$ ) to straight-line (on logarithmic scale) portions of the unsupported ${ }^{210} \mathrm{~Pb}$ profile. The regressions yielded MARs of 0.152 and $0.089 \mathrm{~g} / \mathrm{cm}^{2} \mathrm{yr}$ for the upper and lower portions of the core, respectively. These MARs, applied beginning at $8 \mathrm{~cm}$ (assuming an instantaneous mixing zone), yield reasonable dates for the lead peak (1973-79), DDT and PCB peaks (1968), and ${ }^{137}$ Cs peak (1962); however, the first (deepest) occurrence of chlordane, DDT, and PCBs was dated as 1938, several years before the synthesis of chlordane and prior to commercial use of DDT. Using the ${ }^{137} \mathrm{Cs}$ peak and assuming the same $8-\mathrm{cm}$ mixing zone yields an MAR of $0.125 \mathrm{~g} / \mathrm{cm}^{2} \mathrm{yr}$ and a closer match between ${ }^{137} \mathrm{Cs}$ and ${ }^{210} \mathrm{~Pb}$ dates (Figure $5 \mathrm{~B}$ ). Based on these interpretations, we concluded that the $\mathrm{CF}: \mathrm{CS}{ }^{210} \mathrm{~Pb}$ model was the best choice for the upper part of the core and that dates estimated for the lower part of the core were less certain.

Corroboration Both ${ }^{137} \mathrm{Cs}$ and ${ }^{210} \mathrm{~Pb}$ models yield reasonable, corroborated results back into the early 1960s; however, apparent disagreement between the pre- $1960{ }^{210} \mathrm{~Pb}$ dates and occurrence of organochlorine compounds causes more uncertainty deeper in the core. Spliethoff and Hemond (1996) had somewhat similar results in dating a core from the main body of Upper Mystic Lake, south of the upper embayment of the lake where our cores were collected. Their core had a log-linear profile of ${ }^{210} \mathrm{~Pb}$ to about $45 \mathrm{~cm}$, then a sharp drop and increase in the variability in unsupported ${ }^{210} \mathrm{~Pb}$ below that depth. They suggested that land disturbance in the watershed in the early 1900 s could be the cause of the unusual ${ }^{210} \mathrm{~Pb}$ profile deeper in the core. They matched trace-element contaminant peaks in the lower part of the core to historical industrial activity in the watershed in 1910-20 and assigned dates that implied a much smaller sedimentation rate prior to about 1940 . While we agree that a smaller sedimentation rate is indicated by ${ }^{210} \mathrm{~Pb}$ prior to about the 1950 s, we feel there is considerable uncertainty in the estimated dates for this part of the core.

\section{Location MYS.2}

MYS.2 One gravity core $49 \mathrm{~cm}$ long analyzed for organic compounds.

Basis Only three samples were analyzed. No dates were assigned. 

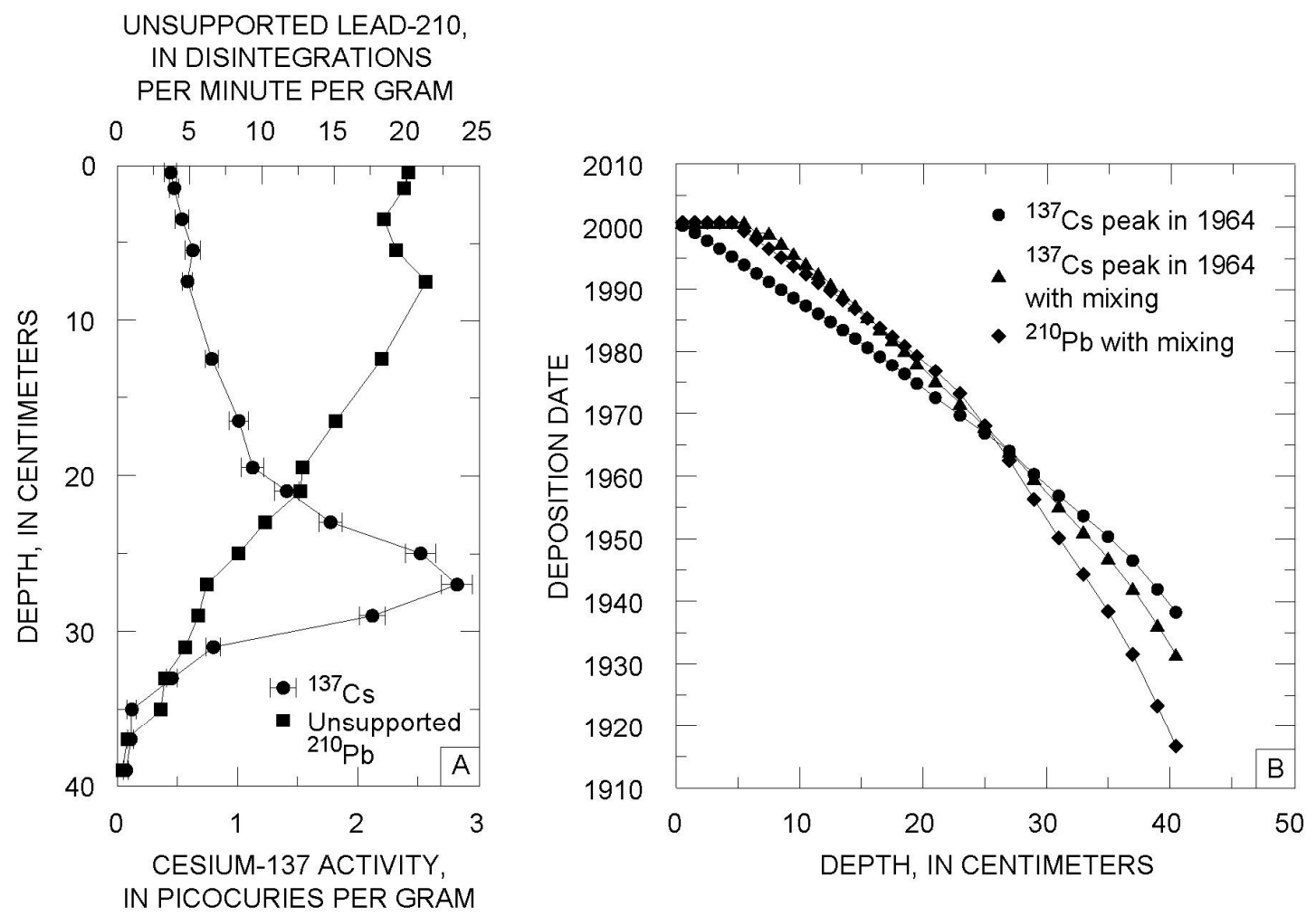

Figure 5. A) Chemical constituent profiles used to estimate age dates for Upper Mystic Lake, Mass. B) Comparison of age dates computed using three different methods for Upper Mystic Lake, Mass.

\section{References}

Appleby, P.G. and Oldfield, F., 1992, Application of lead-210 to sedimentation studies, In: (eds. M. Ivanovich and S. Harmon) Uranium-series Disequilibrium: Applications to Earth, Marine, and Environmental Sciences, 2nd edition: Clarendon Press, Oxford, 910 p.

Spliethoff, H.M. and Hemond, H.F., 1996, History of toxic metal discharge to surface waters of the Aberjona watershed: Environmental Science and Technology, v. 30, p. 121-128.

Van Metre, P.C., Wilson, J.T., Harwell, G.R., Gary, M.O., Heitmuller, F.T., and Mahler, B.J., 2003, Occurrence, trends, and sources in particle-associated contaminants in selected streams and lakes in Fort Worth, Texas: U.S. Geological Survey Water-Resources Investigations Report 03-4169, 154 p. 
Table A1. Radionuclide concentrations and assigned deposition dates for sediment cores.

\begin{tabular}{|c|c|c|c|c|c|c|}
\hline Core ID & $\begin{array}{c}\text { Depth Min. } \\
\text { (cm) }\end{array}$ & $\begin{array}{l}\text { Depth Max. } \\
(\mathbf{c m})\end{array}$ & $\begin{array}{l}\text { Depth Mid. } \\
(\mathbf{c m})\end{array}$ & $\begin{array}{l}\text { Cs-137 } \\
\text { (pCi/g) }\end{array}$ & $\begin{array}{c}\text { 1-sig } \\
\text { (pCi/g) }\end{array}$ & $\begin{array}{c}\text { Deposition } \\
\text { Date }\end{array}$ \\
\hline \multicolumn{7}{|c|}{ Como Lake } \\
\hline \multicolumn{7}{|c|}{ Upper Reservoir Site: } \\
\hline CMO.5 & 0 & 10 & 5.0 & 0.13 & 0.025 & 2000.0 \\
\hline CMO.5 & 30 & 40 & 35.0 & 0.21 & 0.030 & 1990.0 \\
\hline CMO.5 & 40 & 50 & 45.0 & 0.09 & 0.017 & 1965.0 \\
\hline \multicolumn{7}{|c|}{ Middle Reservoir Site: } \\
\hline CMO.3 & 0 & 10 & 5.0 & 0.15 & 0.031 & 1999.0 \\
\hline CMO.3 & 40 & 50 & 45.0 & 0.69 & 0.070 & 1970.0 \\
\hline CMO.3 & 90 & 100 & 95.0 & 0.03 & 0.018 & 1950.0 \\
\hline \multicolumn{7}{|c|}{ Primary (Lower) Reservoir Site: } \\
\hline CMO.1 & 0 & 5 & 2.5 & 0.20 & 0.050 & 2000.8 \\
\hline CMO.1 & 10 & 15 & 12.5 & 0.30 & 0.044 & 1998.7 \\
\hline CMO.1 & 20 & 25 & 22.5 & 0.35 & 0.045 & 1995.3 \\
\hline CMO.1 & 30 & 35 & 32.5 & 0.50 & 0.047 & 1990.6 \\
\hline CMO.1 & 40 & 45 & 42.5 & 0.44 & 0.050 & 1985.3 \\
\hline CMO.1 & 50 & 55 & 52.5 & 0.26 & 0.037 & 1979.6 \\
\hline CMO.1 & 60 & 65 & 62.5 & 0.35 & 0.049 & 1974.1 \\
\hline CMO.1 dup & 60 & 65 & 62.5 & 0.41 & 0.049 & 1974.1 \\
\hline CMO.1 & 70 & 75 & 72.5 & 0.79 & 0.065 & 1969.1 \\
\hline CMO.1 & 80 & 85 & 82.5 & 2.06 & 0.135 & 1964.0 \\
\hline CMO.1 & 90 & 95 & 92.5 & 1.71 & 0.120 & 1958.6 \\
\hline \multicolumn{7}{|c|}{ Echo Lake } \\
\hline \multicolumn{7}{|c|}{ Upper Reservoir Site: } \\
\hline ECO.3 & 0 & 5 & 2.5 & 0.11 & 0.042 & 1999 \\
\hline ECO.3 & 25 & 30 & 27.5 & 0.16 & 0.039 & 1988 \\
\hline ECO.3 & 50 & 55 & 52.5 & 0.60 & 0.056 & 1972 \\
\hline \multicolumn{7}{|c|}{ Middle Reservoir Site: } \\
\hline ECO.4 & 0 & 8 & 4.0 & 0.13 & 0.028 & 1999 \\
\hline ECO.4 & 40 & 48 & 44.0 & 0.70 & 0.060 & 1972 \\
\hline ECO.4 & 72 & 80 & 76.0 & 0.03 & 0.030 & 1952 \\
\hline
\end{tabular}

Primary (Lower) Reservoir Site: 


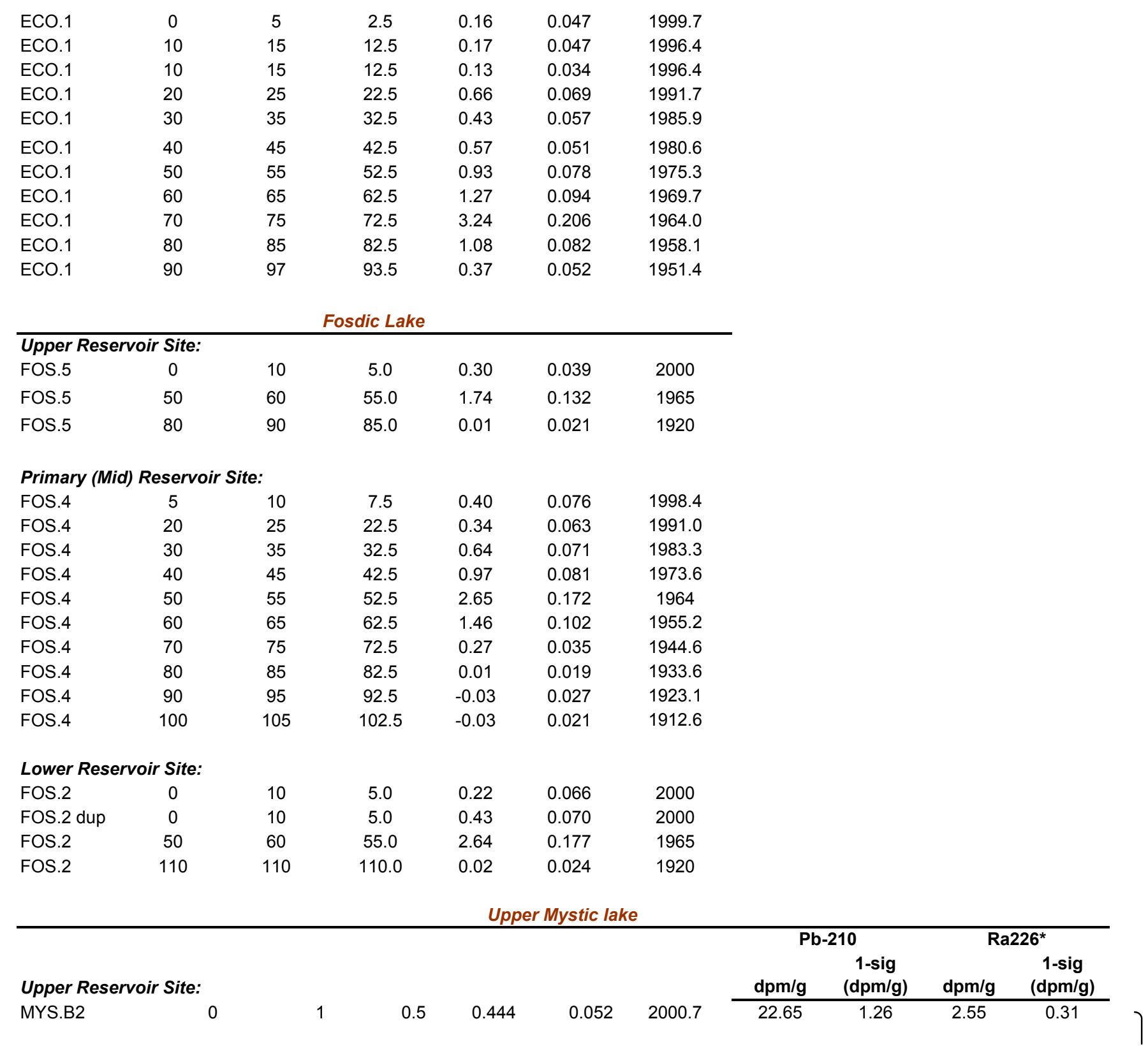




\begin{tabular}{|c|c|c|c|c|c|c|c|c|c|c|c|}
\hline MYS.B2 & 1 & 2 & 1.5 & 0.474 & 0.040 & 2000.7 & 22.64 & 1.12 & 2.84 & 0.24 & Mixed \\
\hline MYS.B2 & 3 & 4 & 3.5 & 0.538 & 0.058 & 2000.7 & 22.53 & 1.29 & 4.10 & 0.37 & Zone \\
\hline MYS.B2 & 7 & 8 & 7.5 & 0.584 & 0.042 & 1999.0 & 23.93 & 1.35 & 2.59 & 0.30 & \\
\hline MYS.B2 & 16 & 17 & 16.5 & 1.011 & 0.082 & 1983.6 & 18.62 & 1.30 & 3.52 & 0.38 & \\
\hline MYS.B2 & 19 & 20 & 19.5 & 1.126 & 0.092 & 1978.1 & 14.81 & 1.19 & 1.99 & 0.46 & \\
\hline MYS.B2 & 20 & 22 & 21 & 1.410 & 0.100 & 1975.2 & 15.24 & 1.15 & 2.59 & 0.36 & \\
\hline MYS.B2 & 26 & 28 & 27 & 2.827 & 0.127 & 1964.0 & 8.43 & 0.59 & 2.24 & 0.19 & \\
\hline MYS.B2 & 28 & 30 & 29 & 2.123 & 0.108 & 1958 & 7.83 & 0.66 & 2.23 & 0.23 & \\
\hline MYS.B2 & 30 & 32 & 31 & 0.799 & 0.059 & 1953 & 6.76 & 0.64 & 2.06 & 0.25 & \\
\hline MYS.B2 & 32 & 34 & 33 & 0.455 & 0.043 & 1948 & 5.94 & 0.55 & 2.65 & 0.24 & \\
\hline MYS.B2 & 34 & 36 & 35 & 0.121 & 0.039 & 1943 & 5.05 & 0.60 & 2.05 & 0.26 & \\
\hline MYS.B2 & 36 & 38 & 37 & 0.112 & 0.024 & 1937 & 2.83 & 0.35 & 2.13 & 0.17 & \\
\hline HSP.B3 & 0 & 3 & 1.5 & 0.55 & 0.065 & 1998.1 & & & & & \\
\hline HSP.B3 & 3 & 6 & 4.5 & 0.64 & 0.073 & 1994.2 & & & & & \\
\hline HSP.B3 & 6 & 9 & 7.5 & 0.64 & 0.067 & 1991.5 & & & & & \\
\hline HSP.B3 & 9 & 12 & 10.5 & 0.69 & 0.084 & 1988.7 & & & & & \\
\hline HSP.B3 & 12 & 15 & 13.5 & 0.70 & 0.084 & 1985.5 & & & & & \\
\hline HSP.B3 & 15 & 18 & 16.5 & 0.61 & 0.069 & 1982.1 & & & & & \\
\hline HSP.B3 & 18 & 21 & 19.5 & 0.70 & 0.071 & 1978.5 & & & & & \\
\hline HSP.B3 & 21 & 24 & 22.5 & 0.60 & 0.062 & 1974.6 & & & & & \\
\hline HSP.B3 & 24 & 27 & 25.5 & 0.65 & 0.074 & 1970.4 & & & & & \\
\hline HSP.B3 & 27 & 30 & 28.5 & 0.84 & 0.068 & 1966.0 & & & & & \\
\hline \multicolumn{12}{|c|}{ Primary (Mid) Reservoir Site: } \\
\hline HSP.B2 & 7 & 8 & 7.5 & 0.71 & & 1992.7 & & & & & \\
\hline HSP.B2 & 8 & 9 & 8.5 & 0.66 & & 1991.4 & & & & & \\
\hline HSP.B2 & 9 & 10 & 9.5 & 0.65 & & 1990.0 & & & & & \\
\hline
\end{tabular}




$\begin{array}{lrrrrrr}\text { HSP.B2 } & 11 & 12 & 11.5 & 0.64 & & 1986.8 \\ \text { HSP.B2 } & 13 & 14 & 13.5 & 0.59 & & 1983.6 \\ \text { HSP.B2 } & 15 & 16 & 15.5 & 0.81 & & 1979.6 \\ \text { HSP.B2 } & 17 & 18 & 17.5 & 0.82 & & 1975.8 \\ \text { HSP.B2 } & 19 & 20 & 19.5 & 0.82 & & 1972.1 \\ \text { HSP.B2 } & 21 & 22 & 21.5 & 0.82 & & 1968.2 \\ \text { HSP.B2 } & 23 & 24 & 23.5 & 1.14 & & 1964.0 \\ \text { HSP.B2 } & 25 & 26 & 25.5 & 0.96 & & 1958.8 \\ \text { HSP.B2 } & 27 & 28 & 27.5 & 0.61 & & 1953.4 \\ & & & & & & \\ \text { Lower Reservoir Site: } & & & & & & \\ \text { HSP.1 } & 0 & 3 & 1.5 & 0.95 & 0.155 & 1997.6 \\ \text { HSP.1 } & 6 & 9 & 7.5 & 1.20 & 0.150 & 1989.1 \\ \text { HSP.1 } & 12 & 15 & 13.5 & 1.10 & 0.110 & 1980.7 \\ \text { HSP.1 } & 15 & 18 & 16.5 & 0.98 & 0.095 & 1975.2 \\ \text { HSP.1 } & 18 & 21 & 19.5 & 1.00 & 0.095 & 1968.8 \\ \text { HSP.1 } & 21 & 24 & 22.5 & 2.36 & 0.185 & 1964.0 \\ \text { HSP.1 } & 24 & 27 & 25.5 & 0.37 & 0.035 & 1955.6 \\ \text { HSP.1 Dup } & 24 & 27 & 25.5 & 0.40 & 0.035 & 1955.6\end{array}$

${ }^{*}$ Ra-226 quantified using average of $\mathrm{Pb}-214$ and $\mathrm{Bi}-214$ daughters. 
Table A2. Suspended sediment concentrations and selected grain size distributions in suspended sediment. Multiple samples are collected during sample processing for a single event composite sample.

\begin{tabular}{|c|c|c|c|c|}
\hline Date & $\begin{array}{c}\text { Suspended } \\
\text { sediment } \\
\text { concentration } \\
\text { (mg/L) }\end{array}$ & $\begin{array}{l}\text { Event Mean } \\
\text { SSC (mg/L) }\end{array}$ & Sand (\%) & $\begin{array}{l}\text { Silt and } \\
\text { clay }(\%)\end{array}$ \\
\hline \multicolumn{5}{|c|}{ Lake Como Inflow } \\
\hline 08/30/01 & 58 & 55 & 15.4 & 84.6 \\
\hline 08/30/01 & 52 & & 12.4 & 87.6 \\
\hline 09/20/01 & 90 & 91.5 & 5.0 & 95.0 \\
\hline 09/20/01 & 93 & & 5.4 & 94.6 \\
\hline $11 / 09 / 01$ & 126 & 133 & 6.9 & 93.1 \\
\hline $11 / 09 / 01$ & 140 & & 7.7 & 92.3 \\
\hline 01/23/02 & 203 & 212 & 4.0 & 96.0 \\
\hline 01/23/02 & 221 & & 3.5 & 96.5 \\
\hline \multicolumn{5}{|c|}{ Echo Lake Inflow } \\
\hline 05/28/01 & 2075 & 2198 & 27.2 & 72.8 \\
\hline 05/28/01 & 2321 & & 37.5 & 62.5 \\
\hline 08/17/01 & 274 & 388.5 & 4.6 & 95.4 \\
\hline 08/17/01 & 503 & & 21.6 & 78.4 \\
\hline 09/20/01 & 197 & 200 & 10.1 & 89.9 \\
\hline 09/20/01 & 203 & & 10.8 & 89.2 \\
\hline 10/11/01 & 465 & 500.5 & 25.2 & 74.8 \\
\hline $10 / 11 / 01$ & 536 & & 27.3 & 72.7 \\
\hline \multicolumn{5}{|c|}{ Fosdic Lake Inflow } \\
\hline 08/11/01 & 188 & 188 & 0.6 & 99.4 \\
\hline 08/11/01 & 188 & & 1.7 & 98.3 \\
\hline 09/18/01 & 816 & 843.5 & 27.2 & 72.8 \\
\hline 09/18/01 & 871 & & 35.7 & 64.3 \\
\hline $10 / 11 / 01$ & 327 & 323 & 4.6 & 95.4 \\
\hline $10 / 11 / 01$ & 319 & & 4.5 & 95.5 \\
\hline $12 / 06 / 01$ & 124 & 125 & 2.3 & 97.7 \\
\hline $12 / 06 / 01$ & 126 & & 3.3 & 96.7 \\
\hline \multicolumn{5}{|c|}{ Aberjona River (Mystic Lake Inflow) } \\
\hline $3 / 21$ to $3 / 22 / 01$ & 41 & 53 & 8 & 92 \\
\hline $3 / 21$ to $3 / 22 / 01$ & 100 & & 8 & 92 \\
\hline $3 / 21$ to $3 / 22 / 01$ & 80 & & 5 & 95 \\
\hline $3 / 22$ to $3 / 23 / 01$ & 31 & & 11 & 89 \\
\hline $3 / 22$ to $3 / 23 / 01$ & 23 & & 5 & 95 \\
\hline $3 / 22$ to $3 / 23 / 01$ & 45 & & 12 & 88 \\
\hline
\end{tabular}




$\begin{array}{lcccc}5 / 22 \text { to } 5 / 24 / 01 & 9 & 9 & 0 & 100 \\ 5 / 22 \text { to } 5 / 24 / 01 & 9 & & 0 & 100 \\ 5 / 22 \text { to } 5 / 24 / 01 & 9 & 18 & 0 & 100 \\ 8 / 29 \text { to } 8 / 30 / 02 & 11 & & 0 & 100 \\ 8 / 29 \text { to } 8 / 30 / 02 & 11 & 6 & 94 \\ 8 / 29 \text { to } 8 / 30 / 02 & 31 & 11 & 33 & 67 \\ & \text { Mill } \text { River (Harris Pond Inflow) } & & \\ 3 / 21 \text { to } 3 / 27 / 01 & 14 & & 22 & 78 \\ 3 / 21 \text { to } 3 / 27 / 01 & 7 & & 38 & 62 \\ 3 / 21 \text { to } 3 / 27 / 01 & 12 & 6 & 4 & 96 \\ 5 / 22 \text { to } 5 / 24 / 01 & 5 & & 29 & 71 \\ 5 / 22 \text { to } 5 / 24 / 01 & 5 & & 3 & 97 \\ 5 / 22 \text { to } 5 / 24 / 01 & 8 & & \end{array}$




\begin{tabular}{|c|c|c|c|c|c|c|c|c|c|c|c|c|c|c|c|c|}
\hline Core ID or Stream & $\begin{array}{l}\text { Depth Min } \\
\text { (cm) }\end{array}$ & $\begin{array}{l}\text { Depth Max } \\
\text { (cm) }\end{array}$ & $\begin{array}{l}\text { Depth Mid } \\
\quad \text { (cm) }\end{array}$ & $\begin{array}{l}\text { Deposition Date } \\
\text { (cores) or } \\
\text { Sample Date } \\
\text { (streams) }\end{array}$ & O.C., \% & Al & $\mathrm{Ca}$ & $\mathrm{Fe}$ & $\mathbf{K}$ & $\mathbf{M g}$ & $\mathrm{Na}$ & $\mathbf{P}$ & $\mathrm{Ti}$ & As & $\mathrm{Ba}$ & $\mathrm{Be}$ \\
\hline \multicolumn{17}{|l|}{ Como Lake } \\
\hline \multicolumn{17}{|l|}{ Upper Reservoir Site: } \\
\hline CMO.5 & 0 & 10 & 5 & 2000 & 5.58 & 31,700 & 156,000 & 19,000 & 6,750 & 4,050 & 1,180 & 946 & 2,030 & 7.6 & 253 & 1.1 \\
\hline CMO.5 & 20 & 30 & 25 & 1990 & 5.31 & 21,700 & 155,000 & 14,000 & 4,960 & 3,020 & 960 & 815 & 1,260 & 7.3 & 205 & 0.75 \\
\hline CMO.5 & 40 & 50 & 45 & 1965 & 0.98 & 8,470 & 273,000 & 11,900 & 1,900 & 2,440 & 823 & 436 & 472 & 5.8 & 98 & 0.40 \\
\hline CMO.5 repl. & 40 & 50 & 45 & 1965 & na & 8,420 & 268,000 & 11,800 & 1,790 & 2,390 & 807 & 437 & 484 & 6.6 & 100 & 0.46 \\
\hline CMO.5 repl. & 40 & 50 & 45 & 1965 & na & 8,430 & 263,000 & 11,500 & 1,830 & 2,360 & 806 & 434 & 460 & 6.5 & 102 & 0.39 \\
\hline \multicolumn{17}{|l|}{ Mid Reservoir Site: } \\
\hline CMO.3 & 0 & 10 & 5 & 1999 & 4.84 & 41,700 & 166,000 & 24,300 & 8,150 & 4,970 & 1,080 & 1,150 & 2,210 & 11 & 297 & 1.3 \\
\hline CMO.3 & 40 & 50 & 45 & 1970 & 4.76 & 42,600 & 184,000 & 25,500 & 8,120 & 5,010 & 1,070 & 1,370 & 2,390 & 12 & 304 & 1.3 \\
\hline CMO. 3 & 90 & 100 & 95 & 1950 & 2.61 & 65,500 & 142,000 & 42,000 & 10,600 & 6,860 & 801 & 967 & 2,720 & 20 & 362 & 2.0 \\
\hline \multicolumn{17}{|c|}{ Primary (Lower Reservoir) Site: } \\
\hline СMO.1 & 0 & 5 & 2.5 & 2000.8 & 5.69 & 49,000 & 168,000 & 28,100 & 8,900 & 5,630 & 1,010 & 1,440 & 2,400 & 14 & 407 & 1.5 \\
\hline CMO.1 & 5 & 10 & 7.5 & 1999.9 & 5.53 & 48,300 & 164,000 & 28,700 & 8,740 & 5,560 & 939 & 1,470 & 2,570 & 15 & 386 & 1.6 \\
\hline CMO.1 & 10 & 15 & 12.5 & 1998.7 & 5.41 & 47,200 & 204,000 & 29,000 & 8,530 & 5,580 & 1,030 & 2,080 & 2,460 & 15 & 396 & 1.6 \\
\hline CMO.1 & 20 & 25 & 22.5 & 1995.3 & 3.62 & 48,800 & 191,000 & 27,400 & 8,920 & 5,640 & 913 & 1,430 & 2,240 & 16 & 318 & 1.7 \\
\hline CMO.1 & 30 & 35 & 32.5 & 1990.6 & 3.42 & 39,700 & 203,000 & 22,200 & 7,960 & 4,710 & 1,100 & 1,070 & 2,240 & 12 & 272 & 1.3 \\
\hline CMO.1 & 45 & 50 & 47.5 & 1982.5 & 3.33 & 35,100 & 204,000 & 20,200 & 7,290 & 4,350 & 1,070 & 968 & 2,250 & 9.5 & 247 & 1.3 \\
\hline CMO.1 & 60 & 65 & 62.5 & 1974.1 & 2.50 & 53,000 & 205,000 & 27,800 & 10,700 & 5,990 & 750 & 1,130 & 2,740 & 13 & 304 & 1.7 \\
\hline CMO.1 & 75 & 80 & 77.5 & 1969.1 & 5.21 & 47,500 & 178,000 & 29,200 & 8,320 & 5,500 & 796 & 1,810 & 2,280 & 17 & 364 & 1.6 \\
\hline CMO.1 & 90 & 95 & 92.5 & 1958.6 & 2.79 & 55,800 & 179,000 & 31,000 & 9,060 & 6,170 & 734 & 1,170 & 2,530 & 18 & 338 & 1.8 \\
\hline \multicolumn{17}{|l|}{ Como Lake Inflow: } \\
\hline Lake Como Inflow & & & & $08 / 30 / 01$ & na & 41,800 & 126,000 & 25,100 & 8,400 & 6,110 & 1,550 & 2,440 & 3,040 & 9.8 & 368 & 1.5 \\
\hline Lake Como Inflow & & & & $09 / 20 / 01$ & na & 38,000 & 147,000 & 24,200 & 8,150 & 5,120 & 1,590 & 2,310 & 1,900 & 12 & 335 & 1.3 \\
\hline Lake Como Inflow & & & & $11 / 09 / 01$ & na & 40,900 & 124,000 & 24,800 & 8,570 & 5,430 & 1,340 & 2,000 & 2,650 & 11 & 344 & 1.5 \\
\hline Lake Como Inflow & & & & $01 / 23 / 02$ & na & 39,800 & 185,000 & 24,000 & 7,660 & 5,950 & 1,100 & 1,350 & 1,920 & 10 & 300 & 1.5 \\
\hline Lake Como Inflow, dup. & & & & $01 / 23 / 02$ & na & 39,200 & 183,000 & 23,700 & 7,560 & 5,890 & 1,080 & 1,370 & 1,960 & 11 & 296 & 1.5 \\
\hline \multicolumn{17}{|l|}{ Echo Lake } \\
\hline \multicolumn{17}{|l|}{ Upper Reservoir Site: } \\
\hline ECO.3 0-5 & 0 & 5 & 2.5 & 1999 & 3.60 & 34,000 & 90,300 & 18,900 & 5,820 & 6,590 & 1,760 & 649 & 2,570 & 10 & 290 & 1.7 \\
\hline ECO.3 25-30 & 25 & 30 & 27.5 & 1988 & 3.54 & 34,400 & 91,600 & 18,200 & 6,180 & 6,350 & 1,860 & 573 & 2,400 & 11 & 283 & 1.7 \\
\hline ECO.3 50-55 & 50 & 55 & 57.5 & 1972 & 3.56 & 30,400 & 91,100 & 16,900 & 5,590 & 5,750 & 2,020 & 667 & 2,240 & 13 & 302 & 1.8 \\
\hline
\end{tabular}


ECO.4 0-8

ECO. 4 40-48

ECO. 4 72-80

ECO.1 0-5

ECO. $10-5$ repl

ECO. $10-5$ repl

ECO.1 5-10

ECO. 1 10-15

ECO. 1 20-25

ECO. $130-35$

ECO. $140-45$

ECO. 1 55-60

ECO. $165-70$

ECO.175-80

ECO. 1 90-97

\section{Echo Lake Inflow:}

\section{Echo Lake Inflow}

Echo Lake Inflow

Echo Lake Inflow

Echlo Lake Inflow

\section{Fosdic Lake}

Upper Reservoir Site:

FOS.5 0-10

FOS.5 50-60

FOS.5 80-90

50

80

Primary (Mid) Reservoir Site:

FOS.4 0-5

FOS.4 0-5 repl

FOS.4 0-5 repl

FOS.4 5-10

FOS. 4 10-15

FOS. 4 20-25

FOS.4 30-35

FOS. 4 45-50

FOS. 4 55-60

FOS.4 55-60 repl

FOS. 4 55-60 repl

FOS.4 70-75

FOS. 4 85-90
8

5
10

25

35

60

96
1999

1972

1952

1999.7

1999.7

1999.7

1998.3

1996.4

1991.7

1985.9

1980.6

1972.5

1966.8

1961.1

1951.4

05/28/0

$08 / 17 / 01$

09/20/01

$10 / 11 / 01$ $\begin{array}{llllllllllll}3.87 & 45,900 & 107,000 & 26,500 & 7,390 & 7,410 & 1,470 & 847 & 2,980 & 13 & 348 & 2.0\end{array}$

$\begin{array}{llllllllllll}4.13 & 46,600 & 109,000 & 25,800 & 7,570 & 7,630 & 1,490 & 828 & 2,780 & 17 & 336 & 2.3\end{array}$

$\begin{array}{llllllllllll}2.76 & 58,000 & 72,400 & 33,200 & 9,300 & 8,510 & 1,500 & 630 & 3,210 & 14 & 376 & 2.7\end{array}$

$\begin{array}{llllllllllll}3.50 & 57,800 & 103,000 & 31,900 & 8,910 & 7,590 & 1,070 & 1,010 & 3,220 & 16 & 413 & 2.4\end{array}$

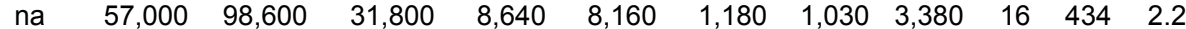

$\begin{array}{llllllllllll}\text { na } & 55,600 & 96,800 & 31,100 & 8,440 & 7,970 & 1,160 & 1,010 & 3,220 & 16 & 422 & 2.2\end{array}$

$\begin{array}{llllllllllll}3.61 & 58,200 & 109,000 & 32,500 & 8,830 & 7,420 & 1,070 & 1,040 & 3,170 & 16 & 400 & 2.3\end{array}$

$\begin{array}{llllllllllll}3.63 & 56,500 & 110,000 & 32,000 & 9,070 & 6,920 & 987 & 982 & 2,900 & 16 & 386 & 2.2\end{array}$

$\begin{array}{llllllllllll}3.74 & 61,400 & 111,000 & 31,000 & 9,680 & 8,150 & 1,130 & 832 & 2,880 & 16 & 366 & 2.4\end{array}$

$\begin{array}{llllllllllll}3.93 & 58,800 & 114,000 & 31,100 & 8,940 & 7,810 & 1,010 & 915 & 2,720 & 18 & 375 & 2.3\end{array}$

$\begin{array}{llllllllllll}3.87 & 58,200 & 113,000 & 31,800 & 9,120 & 8,010 & 1,110 & 964 & 2,680 & 18 & 385 & 2.4\end{array}$

$\begin{array}{llllllllllll}3.92 & 56,500 & 113,000 & 31,400 & 8,970 & 8,170 & 1,070 & 1,160 & 2,730 & 21 & 373 & 2.4\end{array}$

$\begin{array}{llllllllllll}3.41 & 63,100 & 96,700 & 32,800 & 10,400 & 8,950 & 1,200 & 1,570 & 3,130 & 20 & 399 & 2.7\end{array}$

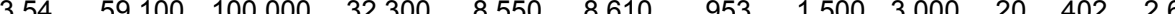

$\begin{array}{llllllllllll}2.91 & 64,700 & 76,600 & 34,300 & 9,780 & 9,710 & 1,240 & 750 & 3,440 & 18 & 412 & 3.0\end{array}$

$\begin{array}{llllllllllll}2.98 & 37,700 & 118,000 & 21,800 & 6,710 & 4,660 & 1,220 & 538 & 2,060 & 7.6 & 303 & 1.3\end{array}$

$\begin{array}{llllllllllll}4.11 & 54,700 & 132,000 & 29,000 & 10,200 & 6,530 & 1,120 & 840 & 2,710 & 12 & 345 & 1.9\end{array}$

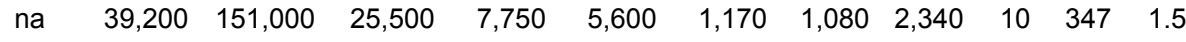

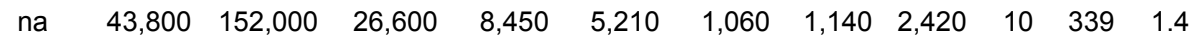

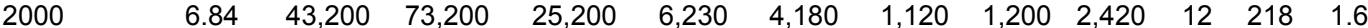

1920

2000.4

2000.4

2000.4

1998.4

1996.2

1991.0

1983.3

1968.9

1959.8

1959.8

1959.8

1944.6

1928.3

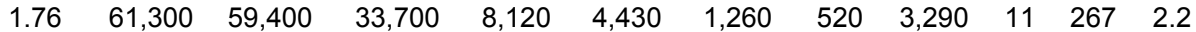

$\begin{array}{llllllllllll}2.67 & 51,000 & 94,600 & 29,900 & 7,670 & 4,430 & 1,050 & 1,620 & 2,990 & 13 & 260 & 1.5\end{array}$

$\begin{array}{llllllllllll}\text { na } & 48,900 & 86,300 & 27,900 & 6,660 & 4,570 & 1,060 & 1,480 & 2,500 & 12 & 248 & 1.8\end{array}$

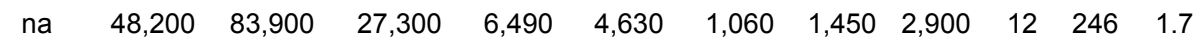

$\begin{array}{llllllllllll}2.62 & 47,600 & 84,900 & 26,900 & 6,880 & 4,540 & 1,080 & 1,500 & 2,270 & 12 & 245 & 1.6\end{array}$

$\begin{array}{llllllllllll}2.49 & 42,500 & 83,400 & 19,200 & 6,700 & 3,950 & 1,000 & 1,300 & 2,310 & 11 & 233 & 1.4\end{array}$

$\begin{array}{llllllllllll}2.21 & 46,900 & 72,600 & 25,500 & 7,120 & 4,410 & 1,110 & 1,310 & 2,330 & 12 & 243 & 1.8\end{array}$

$\begin{array}{llllllllllll}2.66 & 52,300 & 84,900 & 27,200 & 7,780 & 4,720 & 1,040 & 1,140 & 2,500 & 12 & 253 & 1.8\end{array}$

$\begin{array}{llllllllllll}2.59 & 60,700 & 85,000 & 31,600 & 9,390 & 5,100 & 1,160 & 1,260 & 2,950 & 15 & 277 & 1.9\end{array}$

$\begin{array}{llllllllllll}2.97 & 60,200 & 93,400 & 31,000 & 8,150 & 5,140 & 1,070 & 781 & 3,060 & 220 & 253 & 2.2\end{array}$

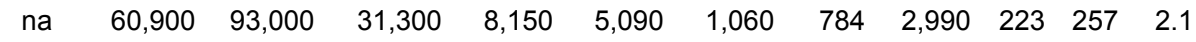

$\begin{array}{llllllllllll}\text { na } & 60,500 & 92,900 & 31,000 & 8,140 & 5,080 & 1,060 & 775 & 3,010 & 218 & 255 & 2.1\end{array}$

$\begin{array}{llllllllllll}1.91 & 69,400 & 67,900 & 36,900 & 8,960 & 5,160 & 1,130 & 666 & 3,560 & 32 & 286 & 2.4\end{array}$

$\begin{array}{llllllllllll}2.34 & 72,000 & 67,000 & 36,500 & 9,640 & 5,350 & 1,140 & 633 & 3,940 & 28 & 288 & 2.3\end{array}$ $\begin{array}{llllllllllll}3.86 & 47,600 & 78,400 & 25,700 & 7,170 & 4,170 & 1,260 & 923 & 2,760 & 14 & 235 & 1.8\end{array}$ 


\begin{tabular}{|c|c|c|c|c|c|c|c|c|c|c|c|c|c|c|c|c|}
\hline FOS.4 100-105 & 100 & 105 & 102.5 & 1912.6 & 2.04 & 66,600 & 42,100 & 30,800 & 8,570 & 5,430 & 1,320 & 547 & 4,050 & 21 & 866 & 2.0 \\
\hline FOS.4 105-112 & 105 & 112 & 108.5 & 1900.0 & 2.20 & 52,200 & 41,200 & 27,100 & 8,240 & 3,920 & 1,120 & 475 & 3,070 & 12 & 276 & 2.0 \\
\hline \multicolumn{17}{|c|}{ Lower Reservoir Site: } \\
\hline FOS.2 0-10 & 0 & 10 & 5 & 2000 & 8.27 & 51,100 & 88,900 & 29,500 & 7,000 & 4,750 & 915 & 1,650 & 2,590 & 15 & 272 & 1.7 \\
\hline FOS.2 50-60 & 50 & 60 & 55 & 1965 & 3.08 & 67,100 & 80,400 & 34,200 & 9,520 & 5,770 & 1,030 & 998 & 3,470 & 18 & 285 & 2.2 \\
\hline FOS.2 100-110 & 100 & 110 & 105 & 1920 & 2.25 & 80,000 & 50,000 & 39,100 & 9,660 & 5,670 & 1,030 & 680 & 4,070 & 18 & 302 & 2.8 \\
\hline \multicolumn{17}{|l|}{ Fosdic Lake Inflow } \\
\hline Fosdic Lake Inflow & & & & 08/11/01 & na & 68,800 & 33,800 & 31,500 & 12,800 & 6,810 & 2,570 & 5,530 & 3,440 & 13 & 278 & 2.8 \\
\hline Fosdic Lake Inflow & & & & 09/18/01 & 4.81 & 78,000 & 50,800 & 37,600 & 8,980 & 5,110 & 1,360 & 1,120 & 4,080 & 13 & 288 & 2.7 \\
\hline Fosdic Lake Inflow & & & & $10 / 10 / 01$ & 8.25 & 68,400 & 101,000 & 33,800 & 8,920 & 4,760 & 905 & 1,290 & 3,380 & 12 & 280 & 2.0 \\
\hline Fosdic Lake Inflow & & & & $12 / 16 / 01$ & na & 91,800 & 56,500 & 42,800 & 10,200 & 6,640 & 1,810 & 1,780 & 4,600 & 13 & 286 & 2.6 \\
\hline \multicolumn{17}{|c|}{ Upper Mystic Lake } \\
\hline \multicolumn{17}{|c|}{ Upper Reservoir Site: } \\
\hline MYS.B2 0-1 & 0.0 & 1.0 & 0.5 & 2000.7 & na & 74,500 & 14,000 & 49,800 & 15,800 & 11,300 & 10,400 & 2,200 & 4,470 & 80 & 500 & na \\
\hline MYS.B2 1-2 & 1.0 & 2.0 & 1.5 & 2000.7 & na & 75,700 & 13,800 & 49,200 & 15,700 & 11,300 & 10,500 & 2,200 & 4,590 & 95 & 524 & na \\
\hline MYS.B2 2-3 & 2.0 & 3.0 & 2.5 & 2000.7 & na & 77,800 & 13,800 & 49,200 & 16,000 & 11,600 & 10,500 & 2,200 & 4,590 & 97 & 544 & na \\
\hline MYS.B2 3-4 & 3.0 & 4.0 & 3.5 & 2000.7 & na & 76,000 & 14,000 & 47,900 & 15,800 & 11,600 & 10,400 & 2,300 & 4,590 & 103 & 544 & na \\
\hline MYS.B2 3-4 Dup & 3.0 & 4.0 & 3.5 & 2000.7 & na & 75,500 & 13,900 & 47,800 & 15,800 & 11,100 & 10,200 & 2,300 & 4,470 & 102 & 528 & na \\
\hline MYS.B2 4-5 & 4.0 & 5.0 & 4.5 & 2000.7 & na & 74,800 & 13,700 & 48,400 & 15,300 & 11,600 & 10,500 & 2,400 & 4,590 & 106 & 518 & na \\
\hline MYS.B2 5-6 & 5.0 & 6.0 & 5.5 & 2000.7 & na & 77,800 & 13,900 & 50,400 & 17,500 & 12,900 & 10,500 & 2,300 & 4,590 & 107 & 606 & na \\
\hline MYS.B2 6-7 & 6.0 & 7.0 & 6.5 & 1998.9 & na & 77,300 & 13,900 & 49,700 & 16,700 & 12,400 & 10,600 & 2,600 & 4,590 & 108 & 562 & na \\
\hline MYS.B2 7-8 & 7.0 & 8.0 & 7.5 & 1999.0 & na & 70,500 & 14,800 & 50,200 & 12,500 & 9,600 & 10,200 & 2,400 & 4,350 & 113 & 412 & na \\
\hline MYS.B2 8-9 & 8.0 & 9.0 & 8.5 & 1997.3 & na & 68,700 & 13,900 & 49,600 & 11,000 & 9,000 & 9,780 & 2,500 & 4,470 & 122 & 365 & na \\
\hline MYS.B2 9-10 & 9.0 & 10.0 & 9.5 & 1995.7 & na & 69,200 & 14,100 & 49,000 & 11,500 & 9,000 & 9,670 & 2,600 & 4,470 & 125 & 389 & na \\
\hline MYS.B2 10-11 & 10.0 & 11.0 & 10.5 & 1994.1 & na & 71,800 & 14,000 & 48,700 & 11,400 & 9,200 & 9,780 & 2,200 & 4,470 & 121 & 386 & na \\
\hline MYS.B2 11-12 & 11.0 & 12.0 & 11.5 & 1992.5 & na & 73,300 & 13,800 & 50,300 & 12,000 & 9,800 & 9,890 & 2,400 & 4,470 & 128 & 389 & na \\
\hline MYS.B2 12-13 & 12.0 & 13.0 & 12.5 & 1990.8 & na & 76,900 & 13,600 & 50,100 & 12,100 & 9,800 & 9,780 & 2,300 & 4,470 & 140 & 383 & na \\
\hline MYS.B2 12-13 Dup & 12.0 & 13.0 & 12.5 & 1989.1 & na & 76,200 & 13,600 & 50,800 & 12,800 & 10,000 & 10,000 & 2,200 & 4,470 & 146 & 402 & na \\
\hline MYS.B2 13-14 & 13.0 & 14.0 & 13.5 & 1989.1 & na & 79,500 & 13,500 & 51,600 & 13,100 & 10,200 & 9,780 & 2,200 & 4,470 & 148 & 408 & na \\
\hline MYS.B2 14-15 & 14.0 & 15.0 & 14.5 & 1987.4 & na & 77,200 & 13,500 & 49,200 & 12,100 & 10,200 & 9,780 & 2,100 & 4,470 & 125 & 378 & na \\
\hline MYS.B2 15-16 & 15.0 & 16.0 & 15.5 & 1985.5 & na & 74,300 & 14,000 & 48,000 & 11,500 & 9,600 & 9,670 & 2,200 & 4,590 & 127 & 354 & na \\
\hline MYS.B2 16-17 & 16.0 & 17.0 & 16.5 & 1983.6 & na & 71,300 & 12,100 & 47,700 & 12,200 & 12,300 & 9,780 & 2,100 & 4,330 & 116 & 338 & na \\
\hline MYS.B2 17-18 & 17.0 & 18.0 & 17.5 & 1981.9 & na & 75,300 & 13,900 & 47,400 & 11,000 & 12,000 & 10,000 & 2,300 & 4,440 & 125 & 308 & na \\
\hline MYS.B2 18-19 & 18.0 & 19.0 & 18.5 & 1980.1 & na & 77,600 & 13,800 & 49,100 & 11,600 & 13,000 & 10,000 & 2,400 & 4,330 & 128 & 326 & na \\
\hline MYS.B2 19-20 & 19.0 & 20.0 & 19.5 & 1978.1 & na & 75,600 & 13,400 & 48,300 & 11,500 & 11,700 & 9,780 & 2,400 & 4,220 & 130 & 320 & na \\
\hline MYS.B2 20-22 & 20.0 & 22.0 & 21.0 & 1975.2 & na & 76,200 & 13,900 & 49,600 & 11,400 & 11,000 & 10,000 & 2,300 & 4,440 & 124 & 315 & na \\
\hline MYS.B2 22-24 & 22.0 & 24.0 & 23.0 & 1971.6 & na & 73,800 & 13,400 & 51,300 & 10,000 & 11,000 & 9,560 & 2,500 & 4,330 & 130 & 282 & na \\
\hline MYS.B2 24-26 & 24.0 & 26.0 & 25.0 & 1967.9 & na & 82,500 & 13,400 & 53,600 & 12,200 & 11,700 & 9,670 & 2,500 & 4,440 & 149 & 397 & na \\
\hline MYS.B2 26-28 & 26.0 & 28.0 & 27.0 & 1964.0 & na & 84,700 & 13,000 & 48,900 & 13,600 & 12,300 & 10,400 & 2,200 & 4,560 & 138 & 402 & na \\
\hline MYS.B2 28-30 & 28.0 & 30.0 & 29.0 & 1958 & na & 85,100 & 14,000 & 45,100 & 12,100 & 13,700 & 10,400 & 2,100 & 4,780 & 119 & 365 & na \\
\hline
\end{tabular}




\begin{tabular}{|c|c|c|c|c|c|c|c|c|c|c|c|c|c|c|c|c|}
\hline MYS.B2 28-30 Dup & 28.0 & 30.0 & 29.0 & 1953 & na & 83,600 & 13,400 & 45,100 & 11,900 & 11,700 & 10,100 & 2,200 & 4,670 & 119 & 356 & na \\
\hline MYS.B2 30-32 & 30.0 & 32.0 & 31.0 & 1953 & na & 83,800 & 14,200 & 44,100 & 11,400 & 13,200 & 10,400 & 2,200 & 4,940 & 117 & 333 & na \\
\hline MYS.B2 32-34 & 32.0 & 34.0 & 33.0 & 1948 & na & 82,200 & 13,800 & 41,600 & 10,100 & 13,200 & 9,880 & 2,000 & 4,470 & 115 & 311 & na \\
\hline MYS.B2 34-36 & 34.0 & 36.0 & 35.0 & 1943 & na & 84,900 & 13,400 & 40,100 & 10,000 & 12,000 & 9,290 & 1,600 & 4,000 & 130 & 278 & na \\
\hline MYS.B2 36-38 & 36.0 & 38.0 & 37.0 & 1937 & na & 81,100 & 11,600 & 29,600 & 10,000 & 12,000 & 8,470 & 1,200 & 3,530 & 127 & 307 & na \\
\hline MYS.B2 38-40 & 38.0 & 40.0 & 39.0 & 1930 & na & 80,200 & 11,400 & 28,400 & 13,000 & 15,200 & 10,200 & 1,000 & 4,120 & 97 & 349 & na \\
\hline MYS.B2 40-41 & 40 & 41 & 40.5 & 1924 & na & 71,800 & 12,000 & 20,700 & 12,700 & 12,800 & 11,500 & 740 & 3,180 & 31 & 338 & na \\
\hline \multicolumn{17}{|c|}{ Aberjona River (Upper Mystic Lake inflow) } \\
\hline Aberjona River & & & & $3 / 21$ to $3 / 22 / 01$ & na & 66,300 & 13,800 & 52,200 & 16,100 & 13,200 & 13,000 & 2,470 & 5,520 & 80 & 455 & 3.42 \\
\hline Aberjona River & & & & $3 / 22$ to $3 / 23 / 01$ & na & 71,200 & 13,100 & 51,200 & 17,400 & 12,300 & 13,300 & 2,900 & 5,240 & 97 & 488 & 3.66 \\
\hline Aberjona River & & & & $5 / 22$ to $5 / 24 / 01$ & na & 41,200 & 12,000 & 115,000 & 9,790 & 8,660 & 6,370 & 4,210 & 3,330 & 296 & 441 & 2.30 \\
\hline Aberjona River & & & & $8 / 29$ to $8 / 30 / 02$ & na & 48,500 & 15,200 & 80,200 & 11,800 & 9,860 & 12,100 & 4,160 & 3,780 & 270 & 449 & 2.74 \\
\hline Aberjona River Dup & & & & 8/29-30 Dup & na & 48,800 & 15,200 & 80,400 & 11,700 & 9,800 & 11,900 & 4,240 & 3,790 & 271 & 447 & 2.64 \\
\hline \multicolumn{17}{|l|}{ Harris Pond } \\
\hline \multicolumn{17}{|c|}{ Upper Reservoir Site: } \\
\hline HSP.B3 & 0.0 & 3.0 & 1.5 & 1998.1 & 10.3 & 81,400 & 19,400 & 26,400 & 13,600 & 10,000 & 17,100 & 1,500 & 3,880 & 4.9 & 569 & na \\
\hline HSP.B3 & 3.0 & 6.0 & 4.5 & 1994.2 & 9.63 & 83,100 & 17,300 & 24,800 & 14,000 & 7,330 & 16,500 & 1,400 & 3,880 & 4.5 & 543 & na \\
\hline HSP.B3 & 6.0 & 9.0 & 7.5 & 1991.5 & 9.25 & 81,800 & 17,600 & 23,900 & 14,100 & 9,000 & 16,800 & 1,500 & 4,000 & 7.2 & 556 & na \\
\hline HSP.B3 Dup & 6.0 & 9.0 & 7.5 & 1991.5 & 9.12 & 80,500 & 17,400 & 23,800 & 14,000 & 8,330 & 16,800 & 1,300 & 3,880 & 6.8 & 544 & na \\
\hline HSP.B3 & 9.0 & 12.0 & 10.5 & 1988.7 & 9.27 & 89,100 & 19,300 & 24,800 & 14,700 & 10,000 & 17,000 & 1,400 & 4,000 & 5.3 & 568 & na \\
\hline HSP.B3 & 12.0 & 15.0 & 13.5 & 1985.5 & 8.85 & 89,800 & 19,600 & 25,400 & 14,100 & 10,300 & 17,600 & 1,500 & 4,000 & 4.4 & 575 & na \\
\hline HSP.B3 & 15.0 & 18.0 & 16.5 & 1982.1 & 8.25 & 78,000 & 14,900 & 24,800 & 14,500 & 7,670 & 16,900 & 1,500 & 4,120 & 5.6 & 529 & na \\
\hline HSP.B3 & 18.0 & 21.0 & 19.5 & 1978.5 & 8.04 & 82,700 & 20,500 & 25,200 & 13,900 & 9,330 & 18,200 & 1,400 & 4,240 & 3.8 & 541 & na \\
\hline HSP.B3 & 21.0 & 24.0 & 22.5 & 1974.6 & 7.41 & 73,600 & 18,900 & 24,200 & 14,600 & 9,000 & 17,900 & 1,200 & 4,120 & 3.5 & 584 & na \\
\hline HSP.B3 Dup & 21.0 & 24.0 & 22.5 & 1974.6 & 7.40 & 81,600 & 19,400 & 24,600 & 15,000 & 9,670 & 18,500 & 1,300 & 4,240 & 3.2 & 599 & na \\
\hline HSP.B3 & 24.0 & 27.0 & 25.5 & 1970.4 & 8.08 & 78,700 & 20,400 & 22,800 & 13,900 & 8,670 & 18,500 & 1,400 & 4,240 & 4.4 & 536 & na \\
\hline HSP.B3 & 27.0 & 30.0 & 28.5 & 1966.0 & 7.33 & 76,700 & 18,200 & 20,400 & 13,800 & 8,000 & 18,800 & 1,200 & 4,120 & 3.6 & 532 & na \\
\hline \multicolumn{17}{|c|}{ Middle Reservoir Site: } \\
\hline HSP.B2 & 0 & 1 & 0.5 & 2000.4 & 10.8 & 83,800 & 16,700 & 36,500 & 12,200 & 9,330 & 14,000 & 2,300 & 4,000 & 6.0 & 565 & na \\
\hline HSP.B2 & 1 & 2 & 1.5 & 1999.6 & 10.4 & 82,500 & 15,300 & 34,000 & 12,800 & 11,000 & 14,700 & 2,000 & 4,120 & 5.1 & 576 & na \\
\hline HSP.B2 & 2 & 3 & 2.5 & 1998.5 & 10.4 & 70,500 & 12,300 & 31,500 & 12,400 & 9,000 & 13,600 & 2,000 & 4,000 & 5.4 & 513 & na \\
\hline HSP.B2 & 3 & 4 & 3.5 & 1997.4 & 10.4 & 82,800 & 15,000 & 32,200 & 12,500 & 11,000 & 14,300 & 2,000 & 4,000 & 4.0 & 569 & na \\
\hline HSP.B2 & 4 & 5 & 4.5 & 1996.2 & 10.2 & 81,800 & 14,800 & 33,300 & 12,600 & 10,700 & 14,500 & 2,000 & 4,120 & 4.8 & 562 & na \\
\hline HSP.B2 Dup & 4 & 5 & 4.5 & 1996.2 & 10.6 & 79,200 & 12,800 & 31,400 & 12,200 & 10,000 & 13,700 & 1,900 & 3,880 & 4.0 & 542 & na \\
\hline HSP.B2 & 5 & 6 & 5.5 & 1995.1 & 10.0 & 76,500 & 14,700 & 32,600 & 12,600 & 8,670 & 14,900 & 2,200 & 4,120 & 7.9 & 553 & na \\
\hline HSP.B2 & 6 & 7 & 6.5 & 1993.9 & 9.84 & 80,200 & 14,700 & 32,800 & 12,700 & 10,300 & 14,900 & 2,300 & 4,120 & 8.8 & 555 & na \\
\hline HSP.B2 & 7 & 8 & 7.5 & 1992.7 & 9.65 & 82,800 & 14,700 & 33,000 & 13,000 & 10,300 & 14,900 & 2,100 & 4,250 & 8.5 & 575 & na \\
\hline HSP.B2 & 8 & 9 & 8.5 & 1991.4 & 9.29 & 84,000 & 13,700 & 33,100 & 13,600 & 10,500 & 14,700 & 2,200 & 4,500 & 8.3 & 523 & na \\
\hline HSP.B2 Dup & 8 & 9 & 8.5 & 1991.4 & 9.16 & 77,000 & 11,800 & 31,000 & 12,900 & 9,000 & 14,000 & 1,800 & 4,000 & 9.0 & 516 & na \\
\hline HSP.B2 & 9 & 10 & 9.5 & 1990.0 & 9.05 & 95,300 & 14,400 & 32,500 & 13,400 & 9,330 & 14,100 & 1,900 & 4,000 & 9.2 & 548 & na \\
\hline HSP.B2 & 11 & 12 & 11.5 & 1986.8 & 8.20 & 85,000 & 14,300 & 32,000 & 13,000 & 10,700 & 14,900 & 2,000 & 4,000 & 6.8 & 551 & na \\
\hline
\end{tabular}




$\begin{array}{lr}\text { HSP.B2 } & 13 \\ \text { HSP.B2 } & 15 \\ \text { HSP.B2 } & 17 \\ \text { HSP.B2 } & 19 \\ \text { HSP.B2 } & 21 \\ \text { HSP.B2 } & 23 \\ \text { HSP.B2 } & 25 \\ \text { HSP.B2 } & 27 \\ \text { Lower Reservoir Site: } & \\ \text { HSP.1 } & 0.0 \\ \text { HSP.1 } & 3.0 \\ \text { HSP.1 } & 6.0 \\ \text { HSP.1 } & 9.0 \\ \text { HSP.1 } & 12.0 \\ \text { HSP.1 } & 15.0 \\ \text { HSP.1 } & 18.0 \\ \text { HSP.1 Dup } & 18.0 \\ \text { HSP.1 } & 21.0 \\ \text { HSP.1 } & 24.0 \\ \text { HSP.1 } & 27.0 \\ \text { HSP.1 } & 30.0 \\ \text { HSP.1 Dup } & 30.0 \\ \text { HSP.1 } & 33.0 \\ \text { Mill Creek (Harris Pond inflow): } & \end{array}$

Mill Creek (Harris Pond inflow):

Mill Creek

Mill Creek

\begin{tabular}{|c|c|c|c|c|c|c|c|c|c|c|c|c|}
\hline 1983.6 & 8.13 & 87,800 & 14,600 & 32,100 & 13,900 & 10,000 & 15,600 & 2,000 & 4,120 & 7.0 & 567 & na \\
\hline 1979.6 & 8.77 & 89,200 & 14,000 & 31,300 & 13,600 & 9,330 & 15,300 & 2,100 & 4,250 & 4.4 & 543 & na \\
\hline 1975.8 & 9.22 & 87,200 & 13,800 & 31,200 & 13,000 & 9,000 & 14,300 & 2,100 & 4,120 & 4.9 & 535 & na \\
\hline 1972.1 & 9.10 & 84,500 & 13,400 & 28,800 & 12,400 & 8,330 & 14,300 & 2,200 & 4,120 & 4.3 & 523 & 1a \\
\hline 1968.2 & 8.76 & 85,300 & 14,600 & 28,200 & 12,200 & 9,330 & 15,500 & 2,200 & 4,120 & 3.3 & 537 & ha \\
\hline 1964.0 & 8.52 & 69,500 & 12,300 & 25,500 & 12,100 & 8,000 & 15,100 & 2,100 & 4,120 & 4.2 & 513 & la \\
\hline 1958.8 & 8.26 & 81,200 & 12,100 & 24,500 & 13,400 & 7,000 & 15,900 & 1,800 & 4,000 & 3.9 & 524 & 1a \\
\hline 1953.4 & 4.80 & 57,100 & 10,400 & 13,900 & 15,200 & 5,330 & 16,100 & 850 & 2,120 & 2.9 & 501 & na \\
\hline 1997.6 & 9.85 & 93,600 & 18,800 & 30,400 & 12,700 & 10,800 & 14,000 & 2,200 & 3,650 & 8.0 & 678 & าa \\
\hline 1992.6 & 9.74 & 89,400 & 17,700 & 30,100 & 12,400 & 9,430 & 13,400 & 2,200 & 3,530 & 8.1 & 651 & na \\
\hline 1989.1 & 8.97 & 90,900 & 18,800 & 28,700 & 12,500 & 10,300 & 14,800 & 1,900 & 3,760 & 9.3 & 642 & na \\
\hline 1985.3 & 8.59 & 97,100 & 21,300 & 27,900 & 12,400 & 10,000 & 15,400 & 1,900 & 4,000 & 16 & 602 & na \\
\hline 1980.7 & 8.81 & 88,700 & 19,200 & 25,400 & 11,200 & 7,430 & 15,900 & 2,000 & 3,880 & 15 & 568 & na \\
\hline 1975.2 & 8.01 & 71,300 & 14,800 & 24,300 & 12,000 & 6,000 & 16,300 & 1,700 & 3,800 & 15 & 543 & a \\
\hline 1968.8 & 8.65 & 82,200 & 16,500 & 21,800 & 11,300 & 6,570 & 14,800 & 1,500 & 3,650 & 13 & 543 & na \\
\hline 1968.8 & 8.83 & 97,400 & 17,200 & 21,300 & 11,200 & 7,100 & 15,800 & 1,400 & 3,650 & 14 & 548 & na \\
\hline 1964.0 & na & 80,400 & 15,700 & 23,100 & 9,530 & 7,710 & 12,000 & 1,600 & 3,060 & 16 & 528 & na \\
\hline 1955.6 & 2.79 & 74,400 & 13,200 & 13,200 & 12,200 & 4,290 & 16,200 & 370 & 2,350 & 3.7 & 506 & na \\
\hline$t$ assigned & 3.36 & 72,700 & 13,500 & 13,600 & 13,400 & 4,290 & 16,800 & 230 & 2,590 & 3.5 & 506 & na \\
\hline$t$ assigned & 2.66 & 72,900 & 10,300 & 16,300 & 13,900 & 4,100 & 16,400 & 280 & 3,060 & 0.90 & 569 & na \\
\hline assigned & 1.67 & 70,200 & 10,500 & 14,900 & 13,400 & 3,430 & 15,900 & 280 & 2,940 & 0.80 & 549 & na \\
\hline t assigned & 3.96 & 90,500 & 14,000 & 17,300 & 17,000 & 4,290 & 17,100 & 320 & 3,060 & 0.80 & 678 & na \\
\hline & na & 55,800 & 14,200 & 23,800 & 13,200 & 5,650 & 15,800 & 1,690 & 3,970 & 6.1 & 503 & 2.51 \\
\hline to $5 / 24 / 01$ & na & 41,200 & 11,900 & 50,600 & 8,390 & 4,150 & 7,160 & 3,270 & 2,920 & 15 & 494 & 2.42 \\
\hline
\end{tabular}




\begin{tabular}{|c|c|c|c|c|c|c|c|c|c|c|c|c|c|c|c|}
\hline Core ID or Stream & Date & Cd & Co & $\mathrm{Cr}$ & $\mathrm{Cu}$ & $\mathrm{Hg}$ & $\mathbf{L i}$ & Mn & $\mathbf{N i}$ & $\mathrm{Pb}$ & Sc & $\mathrm{Sr}$ & $\mathbf{V}$ & $\mathrm{Zn}$ & Remarks \\
\hline \multicolumn{16}{|l|}{ Como Lake } \\
\hline \multicolumn{16}{|l|}{ Upper Reservoir Site: } \\
\hline CMO.5 & 2000 & 0.97 & 6.8 & 51 & 36 & 0.09 & 21 & 447 & 22 & 131 & 5.7 & 280 & 55 & 229 & \\
\hline CMO.5 & 1990 & 1.1 & 5.9 & 41 & 32 & 0.10 & 13 & 297 & 18 & 144 & 3.9 & 244 & 38 & 193 & \\
\hline СMO.5 & 1965 & 0.46 & 3.8 & 29 & 9.3 & 0.05 & 5.7 & 367 & 18 & 112 & 2.1 & 379 & 28 & 52 & \\
\hline CMO.5 repl. & 1965 & 0.47 & 3.7 & 27 & 9.1 & na & 5.5 & 362 & 18 & 108 & 2.1 & 386 & 28 & 53 & \\
\hline CMO.5 repl. & 1965 & 0.47 & 3.6 & 28 & 9.0 & na & 5.4 & 354 & 18 & 111 & 2.1 & 379 & 27 & 51 & \\
\hline \multicolumn{16}{|l|}{ Mid Reservoir Site: } \\
\hline CMO. 3 & 1999 & 1.0 & 8.3 & 62 & 39 & 0.10 & 27 & 568 & 26 & 127 & 7.4 & 280 & 73 & 247 & \\
\hline CMO.3 & 1970 & 2.0 & 8.6 & 96 & 52 & 0.22 & 25 & 587 & 29 & 620 & 7.5 & 278 & 71 & 291 & \\
\hline CMO. 3 & 1950 & 0.63 & 12 & 70 & 23 & 0.09 & 38 & 1,100 & 31 & 77 & 11 & 169 & 112 & 132 & \\
\hline \multicolumn{16}{|c|}{ Primary (Lower Reservoir) Site: } \\
\hline СMO.1 & 2000.8 & 1.2 & 8.8 & 68 & 39 & 0.12 & 32 & 718 & 29 & 124 & 8.3 & 305 & 84 & 262 & \\
\hline CMO.1 & 1999.9 & 1.5 & 9.4 & 72 & 46 & 0.12 & 30 & 685 & 31 & 156 & 8.3 & 292 & 85 & 291 & \\
\hline CMO.1 & 1998.7 & 1.7 & 9.2 & 77 & 54 & 0.13 & 29 & 769 & 30 & 186 & 8.2 & 341 & 80 & 293 & \\
\hline CMO.1 & 1995.3 & 1.3 & 9.3 & 74 & 34 & 0.14 & 31 & 696 & 29 & 195 & 8.3 & 261 & 84 & 217 & \\
\hline CMO.1 & 1990.6 & 1.3 & 8.3 & 91 & 34 & 0.14 & 23 & 565 & 26 & 410 & 7.2 & 264 & 68 & 199 & \\
\hline CMO.1 & 1982.5 & 1.0 & 7.8 & 70 & 32 & 0.15 & 21 & 504 & 25 & 241 & 6.3 & 282 & 60 & 171 & \\
\hline CMO.1 & 1974.1 & 0.90 & 9.7 & 77 & 30 & 0.10 & 31 & 682 & 30 & 159 & 9.2 & 276 & 91 & 164 & \\
\hline CMO.1 & 1969.1 & 2.3 & 9.4 & 118 & 46 & 0.17 & 29 & 745 & 30 & 1,220 & 8.1 & 273 & 82 & 319 & \\
\hline CMO.1 & 1958.6 & 1.2 & 11 & 120 & 24 & 0.09 & 33 & 828 & 31 & 303 & 9.5 & 211 & 97 & 168 & \\
\hline \multicolumn{16}{|l|}{ Como Lake Inflow: } \\
\hline Lake Como Inflow & 08/30/01 & 2.7 & 9.9 & 74 & 104 & na & 26 & 1,110 & 30 & 147 & 7.7 & 284 & 73 & 637 & \\
\hline Lake Como Inflow & 09/20/01 & 1.9 & 9.1 & 64 & 86 & na & 22 & 1,230 & 28 & 133 & 6.9 & 290 & 67 & 657 & \\
\hline Lake Como Inflow & $11 / 09 / 01$ & 1.9 & 9.2 & 65 & 95 & na & 24 & 559 & 28 & 172 & 7.4 & 316 & 71 & 590 & \\
\hline Lake Como Inflow & $01 / 23 / 02$ & 1.5 & 9.0 & 57 & 64 & 0.13 & 23 & 820 & 29 & 121 & 6.7 & 327 & 68 & 421 & \\
\hline Lake Como Inflow, c & $01 / 23 / 02$ & 1.4 & 8.9 & 56 & 64 & na & 23 & 816 & 29 & 121 & 6.6 & 326 & 68 & 412 & \\
\hline \multicolumn{16}{|l|}{ Echo Lake } \\
\hline \multicolumn{16}{|l|}{ Upper Reservoir Site: } \\
\hline ECO.3 0-5 & 1999 & 2.8 & 7.7 & 54 & 44 & 0.09 & 28 & 446 & 26 & 137 & 6.3 & 232 & 55 & 292 & \\
\hline ECO.3 25-30 & 1988 & 4.5 & 8.0 & 56 & 45 & 0.14 & 28 & 336 & 22 & 185 & 6.2 & 226 & 56 & 265 & \\
\hline ECO.3 50-55 & 1972 & 12 & 7.0 & 93 & 40 & 0.12 & 25 & 335 & 21 & 721 & 5.7 & 191 & 49 & 241 & \\
\hline
\end{tabular}




\begin{tabular}{|c|c|c|c|c|c|c|c|c|c|c|c|c|c|c|}
\hline ECO.4 0-8 & 1999 & 3.2 & 9.0 & 63 & 57 & 0.10 & 35 & 636 & 29 & 134 & 8.0 & 248 & 74 & 307 \\
\hline ECO.4 40-48 & 1972 & 19 & 8.9 & 86 & 47 & 0.15 & 37 & 528 & 26 & 742 & 8.0 & 221 & 72 & 336 \\
\hline ECO.4 72-80 & 1952 & 0.98 & 9.4 & 63 & 23 & 0.07 & 47 & 958 & 24 & 78 & 10 & 160 & 83 & 108 \\
\hline \multicolumn{15}{|c|}{ Primary (Lower) Reservoir Site: } \\
\hline ECO. $10-5$ & 1999.7 & 3.2 & 11 & 72 & 40 & 0.11 & 42 & 731 & 31 & 124 & 9.9 & 238 & 88 & 297 \\
\hline ECO.1 0-5 repl & 1999.7 & 3.3 & 10 & 72 & 40 & na & 43 & 734 & 30 & 126 & 9.7 & 237 & 90 & 292 \\
\hline ECO.1 0-5 repl & 1999.7 & 3.3 & 10 & 70 & 39 & na & 42 & 720 & 29 & 124 & 9.5 & 231 & 88 & 287 \\
\hline ECO.15-10 & 1998.3 & 4.8 & 11 & 75 & 46 & 0.14 & 42 & 685 & 32 & 144 & 9.8 & 241 & 88 & 352 \\
\hline ECO.1 10-15 & 1996.4 & 4.2 & 10 & 73 & 39 & 0.13 & 40 & 633 & 30 & 129 & 10 & 230 & 85 & 305 \\
\hline ECO.1 20-25 & 1991.7 & 6.6 & 11 & 74 & 39 & 0.15 & 46 & 634 & 31 & 165 & 10 & 232 & 89 & 276 \\
\hline ECO.130-35 & 1985.9 & 55 & 10 & 101 & 44 & 0.14 & 42 & 668 & 29 & 303 & 9.6 & 233 & 86 & 519 \\
\hline ECO.1 40-45 & 1980.6 & 16 & 10 & 84 & 43 & 0.14 & 44 & 673 & 30 & 464 & 9.8 & 223 & 86 & 322 \\
\hline ECO.1 55-60 & 1972.5 & 33 & 10 & 136 & 43 & 0.15 & 44 & 729 & 32 & 706 & 9.6 & 232 & 84 & 315 \\
\hline ECO.1 65-70 & 1966.8 & 182 & 10 & 240 & 54 & 0.14 & 51 & 728 & 38 & 305 & 10 & 204 & 88 & 351 \\
\hline ECO.1 75-80 & 1961.1 & 81 & 9.9 & 114 & 45 & 0.12 & 45 & 771 & 29 & 307 & 9.8 & 199 & 84 & 215 \\
\hline ECO.1 90-97 & 1951.4 & 2.0 & 10 & 70 & 25 & 0.09 & 52 & 791 & 27 & 111 & 11 & 172 & 90 & 129 \\
\hline \multicolumn{15}{|l|}{ Echo Lake Inflow: } \\
\hline Echo Lake Inflow & $05 / 28 / 01$ & 1.3 & 7.4 & 55 & 41 & 0.07 & 28 & 401 & 24 & 80 & 7.0 & 432 & 54 & 269 \\
\hline Echo Lake Inflow & 08/17/01 & 2.4 & 11 & 68 & 43 & 0.11 & 40 & 627 & 36 & 146 & 10.0 & 264 & 76 & 375 \\
\hline Echo Lake Inflow & 09/20/01 & 2.7 & 10 & 60 & 62 & na & 27 & 858 & 35 & 166 & 7.4 & 322 & 62 & 607 \\
\hline Echlo Lake Inflow & $10 / 11 / 01$ & 2.4 & 10 & 69 & 60 & na & 30 & 733 & 34 & 212 & 8.0 & 334 & 68 & 554 \\
\hline \multicolumn{15}{|c|}{ Fosdic Lake } \\
\hline \multicolumn{15}{|c|}{ Upper Reservoir Site: } \\
\hline FOS.5 0-10 & 2000 & 1.6 & 9.4 & 64 & 35 & 0.23 & 34 & 648 & 23 & 123 & 7.6 & 187 & 66 & 218 \\
\hline FOS.5 50-60 & 1965 & 2.9 & 9.0 & 74 & 33 & 0.40 & 40 & 448 & 23 & 410 & 8.5 & 148 & 69 & 177 \\
\hline FOS.5 $80-90$ & 1920 & 0.29 & 11 & 61 & 18 & 0.06 & 47 & 989 & 24 & 52 & 11 & 121 & 89 & 79 \\
\hline \multicolumn{15}{|c|}{ Primary (Mid) Reservoir Site: } \\
\hline FOS.4 0-5 & 2000.4 & 1.7 & 10 & 87 & 38 & 0.24 & 37 & 869 & 26 & 108 & 9.2 & 222 & 78 & 223 \\
\hline FOS.4 0-5 repl & 2000.4 & 1.7 & 9.6 & 70 & 36 & na & 37 & 823 & 24 & 108 & 8.9 & 208 & 74 & 208 \\
\hline FOS.4 0-5 repl & 2000.4 & 1.7 & 9.3 & 71 & 35 & na & 37 & 799 & 23 & 111 & 8.7 & 204 & 74 & 203 \\
\hline FOS.4 5-10 & 1998.4 & 2.1 & 9.3 & 74 & 48 & 0.28 & 36 & 774 & 24 & 129 & 8.4 & 202 & 69 & 247 \\
\hline FOS.4 10-15 & 1996.2 & 2.3 & 8.7 & 71 & 42 & 0.31 & 32 & 570 & 23 & 140 & 7.5 & 192 & 62 & 244 \\
\hline FOS.4 20-25 & 1991.0 & 2.8 & 9.0 & 81 & 44 & 0.37 & 37 & 517 & 24 & 196 & 8.5 & 161 & 69 & 248 \\
\hline FOS.4 30-35 & 1983.3 & 3.7 & 9.9 & 86 & 45 & 0.41 & 42 & 494 & 27 & 285 & 9.4 & 159 & 76 & 218 \\
\hline FOS.4 45-50 & 1968.9 & 4.1 & 11 & 96 & 40 & 0.55 & 49 & 592 & 29 & 379 & 11 & 160 & 85 & 206 \\
\hline FOS.4 55-60 & 1959.8 & 0.90 & 11 & 66 & 22 & 0.16 & 48 & 572 & 26 & 243 & 10 & 154 & 90 & 130 \\
\hline FOS.4 55-60 repl & 1959.8 & 0.91 & 11 & 65 & 21 & na & 49 & 576 & 26 & 244 & 10 & 156 & 89 & 130 \\
\hline FOS.4 55-60 repl & 1959.8 & 0.93 & 11 & 65 & 21 & na & 48 & 571 & 25 & 240 & 11 & 156 & 90 & 130 \\
\hline FOS.4 70-75 & 1944.6 & 0.47 & 12 & 71 & 20 & 0.09 & 51 & 834 & 28 & 89 & 12 & 136 & 102 & 100 \\
\hline FOS.4 85-90 & 1928.3 & 0.24 & 12 & 73 & 16 & 0.06 & 55 & 566 & 28 & 39 & 13 & 133 & 104 & 87 \\
\hline
\end{tabular}




\begin{tabular}{|c|c|c|c|c|c|c|c|c|c|c|c|c|c|c|c|}
\hline FOS.4 100-105 & 1912.6 & 0.21 & 11 & 64 & 16 & 0.04 & 52 & 520 & 25 & 27 & 11 & 110 & 89 & 70 & \\
\hline FOS.4 105-112 & 1900.0 & 0.14 & 9.9 & 55 & 14 & 0.04 & 41 & 446 & 22 & 20 & 9.5 & 96 & 76 & 53 & \\
\hline \multicolumn{16}{|c|}{ Lower Reservoir Site: } \\
\hline FOS.2 0-10 & 2000 & 2.0 & 9.1 & 79 & 39 & 0.24 & 39 & 767 & 26 & 105 & 8.8 & 210 & 78 & 225 & \\
\hline FOS.2 50-60 & 1965 & 1.8 & 12 & 80 & 27 & 0.21 & 57 & 594 & 28 & 290 & 12 & 151 & 97 & 149 & \\
\hline FOS.2 100-110 & 1920 & 0.24 & 12 & 79 & 17 & 0.05 & 62 & 533 & 29 & 39 & 14 & 120 & 112 & 92 & \\
\hline \multicolumn{16}{|l|}{ Fosdic Lake Inflow } \\
\hline Fosdic Lake Inflow & $08 / 11 / 01$ & 2.2 & 10 & 89 & 197 & na & 48 & 394 & 24 & 127 & 12 & 153 & 126 & 596 & \\
\hline Fosdic Lake Inflow & 09/18/01 & 0.64 & 13 & 81 & 27 & 0.11 & 59 & 808 & 30 & 72 & 14 & 143 & 106 & 184 & \\
\hline Fosdic Lake Inflow & $10 / 10 / 01$ & 1.1 & 13 & 78 & 40 & 0.13 & 48 & 637 & 35 & 80 & 12 & 278 & 107 & 374 & \\
\hline Fosdic Lake Inflow & $12 / 16 / 01$ & 1.3 & 14 & 93 & 51 & na & 61 & 1,130 & 34 & 84 & 15 & 202 & 116 & 340 & \\
\hline \multicolumn{16}{|l|}{ Upper Mystic Lake } \\
\hline \multicolumn{16}{|c|}{ Upper Reservoir Site: } \\
\hline MYS.B2 0-1 & 2000.7 & 8.2 & 26 & 379 & 390 & 1.8 & 41 & 852 & 48 & 563 & 18 & 149 & 102 & 1,930 & $0-6 \mathrm{~cm}$ mixing zone in core (based on \\
\hline MYS.B2 1-2 & 2000.7 & 8.2 & 23 & 391 & 408 & 1.6 & 41 & 890 & 51 & 690 & 18 & 153 & 111 & 2,030 & $210 \mathrm{~Pb}$ ) assigned sampling date assuming \\
\hline MYS.B2 2-3 & 2000.7 & 8.1 & 27 & 386 & 405 & 1.7 & 42 & 900 & 46 & 666 & 18 & 154 & 108 & 2,010 & instantaneous mixing. \\
\hline MYS.B2 3-4 & 2000.7 & 7.9 & 30 & 372 & 404 & 1.8 & 43 & 918 & 49 & 617 & 17 & 156 & 107 & 1,960 & \\
\hline MYS.B2 3-4 Dup & 2000.7 & 7.8 & 28 & 376 & 398 & 1.8 & 43 & 921 & 50 & 622 & 19 & 146 & 106 & 1,960 & \\
\hline MYS.B2 4-5 & 2000.7 & 8.1 & 23 & 382 & 413 & 1.7 & 41 & 956 & 51 & 635 & 19 & 149 & 115 & 2,000 & \\
\hline MYS.B2 5-6 & 2000.7 & 8.2 & 28 & 395 & 419 & 1.8 & 41 & 969 & 43 & 629 & 18 & 167 & 110 & 2,050 & \\
\hline MYS.B2 6-7 & 1998.9 & 8.2 & 22 & 382 & 411 & 1.8 & 40 & 989 & 48 & 676 & 20 & 156 & 112 & 1,990 & \\
\hline MYS.B2 7-8 & 1999.0 & 7.1 & 29 & 414 & 404 & 1.8 & 43 & 1,000 & 39 & 637 & 20 & 140 & 114 & 2,150 & \\
\hline MYS.B2 8-9 & 1997.3 & 8.3 & 22 & 411 & 402 & 1.7 & 41 & 1,010 & 45 & 623 & 20 & 124 & 110 & 2,180 & \\
\hline MYS.B2 9-10 & 1995.7 & 7.9 & 23 & 464 & 404 & 1.8 & 41 & 1,010 & 66 & 653 & 19 & 128 & 112 & 2,190 & \\
\hline MYS.B2 10-11 & 1994.1 & 7.8 & 36 & 426 & 407 & 1.7 & 42 & 971 & 48 & 688 & 19 & 132 & 109 & 2,230 & \\
\hline MYS.B2 11-12 & 1992.5 & 7.9 & 26 & 459 & 424 & 1.8 & 43 & 951 & 54 & 726 & 20 & 135 & 114 & 2,300 & \\
\hline MYS.B2 12-13 & 1990.8 & 8.3 & 29 & 496 & 440 & 1.7 & 42 & 863 & 51 & 850 & 20 & 134 & 115 & 2,410 & \\
\hline MYS.B2 12-13 Dup & 1989.1 & 8.3 & 30 & 504 & 444 & 1.8 & 43 & 850 & 53 & 848 & 20 & 145 & 116 & 2,430 & \\
\hline MYS.B2 13-14 & 1989.1 & 8.3 & 31 & 544 & 440 & 1.8 & 43 & 804 & 53 & 971 & 19 & 151 & 123 & 2,490 & \\
\hline MYS.B2 14-15 & 1987.4 & 8.2 & 31 & 543 & 433 & 1.8 & 43 & 765 & 53 & 1,000 & 20 & 141 & 119 & 2,500 & \\
\hline MYS.B2 15-16 & 1985.5 & 8.2 & 26 & 564 & 445 & 1.7 & 42 & 730 & 53 & 990 & 20 & 133 & 124 & 2,570 & \\
\hline MYS.B2 16-17 & 1983.6 & 8.5 & 21 & 530 & 461 & 1.7 & 44 & 749 & 52 & 1,110 & 17 & 124 & 124 & 2,660 & \\
\hline MYS.B2 17-18 & 1981.9 & 9.4 & 26 & 564 & 469 & 1.8 & 44 & 749 & 53 & 1,170 & 20 & 120 & 132 & 2,700 & \\
\hline MYS.B2 18-19 & 1980.1 & 9.8 & 33 & 603 & 483 & 1.9 & 46 & 713 & 53 & 1,260 & 20 & 128 & 134 & 2,750 & \\
\hline MYS.B2 19-20 & 1978.1 & 9.5 & 32 & 660 & 496 & 1.8 & 44 & 692 & 56 & 1,460 & 18 & 124 & 134 & 2,890 & \\
\hline MYS.B2 20-22 & 1975.2 & 11 & 32 & 746 & 526 & 1.7 & 43 & 668 & 58 & 1,450 & 16 & 130 & 142 & 2,940 & \\
\hline MYS.B2 22-24 & 1971.6 & 13 & 33 & 958 & 554 & 1.9 & 43 & 637 & 51 & 1,540 & 18 & 120 & 161 & 3,120 & \\
\hline MYS.B2 24-26 & 1967.9 & 12 & 30 & 1,270 & 509 & 1.9 & 43 & 623 & 54 & 1,350 & 13 & 155 & 175 & 3,280 & \\
\hline MYS.B2 26-28 & 1964.0 & 8.8 & 34 & 1,200 & 427 & 1.9 & 46 & 605 & 58 & 1,090 & 13 & 163 & 164 & 3,180 & \\
\hline MYS.B2 28-30 & 1958 & 8.9 & 35 & 952 & 396 & 2.2 & 45 & 605 & 47 & 987 & 19 & 159 & 154 & 3,190 & \\
\hline
\end{tabular}




$\begin{array}{llllllllllllllll}\text { MYS.B2 28-30 Dup } & 1953 & 9.1 & 35 & 950 & 391 & 2.1 & 44 & 600 & 46 & 997 & 17 & 148 & 151 & 3,170 \\ \text { MYS.B2 30-32 } & 1953 & 9.4 & 42 & 552 & 437 & 2.5 & 44 & 593 & 52 & 792 & 18 & 154 & 142 & 3,370 \\ \text { MYS.B2 32-34 } & 1948 & 13 & 34 & 468 & 511 & 2.6 & 40 & 564 & 44 & 718 & 18 & 148 & 120 & 3,670 \\ \text { MYS.B2 34-36 } & 1943 & 14 & 41 & 429 & 727 & 2.6 & 33 & 523 & 35 & 556 & 15 & 138 & 85 & 5,270 \\ \text { MYS.B2 36-38 } & 1937 & 12 & 24 & 281 & 642 & 2.5 & 29 & 451 & 26 & 296 & 15 & 140 & 61 & 5,330 \\ \text { MYS.B2 38-40 } & 1930 & 10 & 31 & 118 & 500 & 0.51 & 28 & 651 & 29 & 164 & 16 & 149 & 60 & 7,090 \\ \text { MYS.B2 40-41 } & 1924 & 6.1 & 24 & 148 & 202 & 0.40 & 18 & 507 & 36 & 50 & 12 & 157 & 51 & 3,210 \\ \text { Aberjona River (Upper Mystic Lake inflow) } & & & & & & & & & & & \\ \text { Aberjona River } & 3 / 21 \text { to 3/22/0.0 } & 4.9 & 22 & 301 & 222 & 1.3 & 48 & 1,580 & 42 & 344 & 12 & 157 & 126 & 857 & \text { Mean of these two samples over 3-day } \\ \text { Aberjona River } & 3 / 22 \text { to 3/23/0 } & 4.4 & 21 & 249 & 190 & \text { na } & 50 & 1,680 & 41 & 312 & 12 & 152 & 120 & 739 \text { event used to represent event. } \\ \text { Aberjona River } & 5 / 22 \text { to 5/24/0.0 } & 6.8 & 45 & 368 & 331 & \text { na } & 33 & 17,000 & 41 & 515 & 8.0 & 128 & 127 & 1,610\end{array}$

Harris Pond

Upper Reservoir Site:

$\begin{array}{lllllllllllllll}\text { HSP.B3 } & 1998.1 & 0.45 & 16 & 37 & 43 & 0.14 & 15 & 631 & 17 & 59 & 11 & 156 & 56 & 203\end{array}$

$\begin{array}{lllllllllllllll}\text { HSP.B3 } & 1994.2 & 0.36 & 14 & 34 & 45 & 0.14 & 14 & 579 & 16 & 63 & 10 & 145 & 60 & 199\end{array}$

$\begin{array}{lllllllllllllll}\text { HSP.B3 } & 1991.5 & 0.39 & 11 & 34 & 47 & 0.16 & 16 & 549 & 14 & 62 & 11 & 150 & 63 & 191\end{array}$

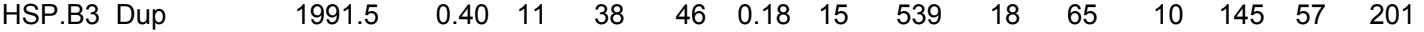

$\begin{array}{lllllllllllllll}\text { HSP.B3 } & 1988.7 & 0.41 & 12 & 44 & 51 & 0.17 & 18 & 559 & 18 & 66 & 11 & 160 & 67 & 194\end{array}$

$\begin{array}{lllllllllllllll}\text { HSP.B3 } & 1985.5 & 0.38 & 14 & 44 & 55 & 0.20 & 15 & 546 & 22 & 69 & 11 & 164 & 63 & 203\end{array}$

$\begin{array}{lllllllllllllll}\text { HSP.B3 } & 1982.1 & 0.38 & 14 & 41 & 53 & 0.16 & 15 & 487 & 14 & 71 & 8.6 & 135 & 63 & 193\end{array}$

$\begin{array}{lllllllllllllll}\text { HSP.B3 } & 1978.5 & 0.35 & 9.4 & 38 & 51 & 0.12 & 17 & 560 & 12 & 70 & 10 & 162 & 68 & 197\end{array}$

$\begin{array}{llllllllllllllll}\text { HSP.B3 } & 1974.6 & 0.32 & 9.3 & 41 & 52 & 0.17 & 17 & 499 & 15 & 62 & 8.0 & 164 & 63 & 167\end{array}$

$\begin{array}{lllllllllllllll}\text { HSP.B3 Dup } & 1974.6 & 0.33 & 10 & 44 & 54 & 0.17 & 16 & 516 & 15 & 61 & 9.8 & 172 & 65 & 176\end{array}$

$\begin{array}{lllllllllllllll}\text { HSP.B3 } & 1970.4 & 0.37 & 11 & 52 & 61 & 0.18 & 16 & 514 & 15 & 46 & 10 & 164 & 63 & 174\end{array}$

$\begin{array}{lllllllllllllll}\text { HSP.B3 } & 1966.0 & 0.42 & 11 & 43 & 72 & 0.17 & 14 & 460 & 14 & 46 & 9.6 & 165 & 65 & 186\end{array}$

Middle Reservoir Site:

$\begin{array}{llllllllllllll}2000.4 & 0.83 & 16 & 35 & 60 & 0.14 & 20 & 952 & 23 & 55 & 17 & 167 & 60 & 213\end{array}$

HSP.B2

1999.6

$\begin{array}{lll}0.88 & 11 & 42\end{array}$

$\begin{array}{llllllllll}58 & 0.20 & 19 & 816 & 20 & 57 & 19 & 161 & 59 & 216\end{array}$

HSP.B2

HSP.B2

HSP.B2

HSP.B2 Dup

HSP.B2

HSP.B2

HSP.B2

HSP.B2

HSP.B2 Dup

HSP.B2

1998.5
1997.4

$\begin{array}{lll}0.53 & 14 & 39\end{array}$

$1996.2 \quad 0.52 \quad 14$

$1996.2 \quad 0.45 \quad 13 \quad 40$

$1995.1 \quad 0.41 \quad 12 \quad 42$

$1993.9 \quad 0.40 \quad 10 \quad 44$

$\begin{array}{llll}1992.7 & 0.57 & 13 & 43\end{array}$

$1991.4 \quad 0.18 \quad 14 \quad 50$

$1991.4 \quad 0.16 \quad 13 \quad 51$

$\begin{array}{llll}1990.0 & 0.15 & 14 & 52\end{array}$

$\begin{array}{llllllllll}54 & 0.21 & 18 & 708 & 26 & 58 & 18 & 135 & 52 & 199\end{array}$

$\begin{array}{llllllllll}55 & 0.22 & 17 & 776 & 15 & 59 & 21 & 160 & 59 & 211\end{array}$

$\begin{array}{llllllllll}55 & 0.21 & 21 & 739 & 12 & 55 & 19 & 159 & 60 & 201\end{array}$

$\begin{array}{llllllllll}54 & 0.20 & 22 & 711 & 11 & 55 & 19 & 151 & 56 & 201\end{array}$

$\begin{array}{llllllllll}60 & 0.20 & 21 & 715 & 10 & 57 & 16 & 160 & 61 & 212\end{array}$

$\begin{array}{llllllllll}60 & 0.21 & 20 & 700 & 12 & 53 & 19 & 156 & 62 & 209\end{array}$

$\begin{array}{llllllllll}60 & 0.23 & 22 & 692 & 14 & 54 & 18 & 160 & 60 & 205\end{array}$

$\begin{array}{llllllllll}62 & 0.22 & 19 & 692 & 22 & 58 & 17 & 153 & 68 & 218\end{array}$

HSP.B2

1986.8

$\begin{array}{llllllllll}61 & 0.21 & 18 & 686 & 20 & 57 & 18 & 148 & 60 & 199\end{array}$

$\begin{array}{llllllllll}61 & 0.22 & 17 & 695 & 21 & 70 & 23 & 157 & 68 & 204\end{array}$

$\begin{array}{llllllllll}63 & 0.25 & 23 & 655 & 20 & 62 & 18 & 155 & 66 & 211\end{array}$ 


\begin{tabular}{|c|c|c|c|c|c|c|c|c|c|c|c|c|c|c|}
\hline HSP.B2 & 1983.6 & 0.29 & 16 & 54 & 64 & 0.21 & 21 & 668 & 24 & 62 & 19 & 161 & 70 & 200 \\
\hline HSP.B2 & 1979.6 & 0.14 & 16 & 50 & 72 & 0.21 & 16 & 639 & 25 & 60 & 20 & 153 & 66 & 195 \\
\hline HSP.B2 & 1975.8 & 0.18 & 12 & 51 & 87 & 0.24 & 16 & 619 & 21 & 62 & 20 & 155 & 61 & 200 \\
\hline HSP.B2 & 1972.1 & 0.24 & 13 & 58 & 102 & 0.23 & 16 & 615 & 25 & 85 & 19 & 153 & 62 & 215 \\
\hline HSP.B2 & 1968.2 & 0.27 & 12 & 53 & 101 & 0.25 & 15 & 595 & 21 & 87 & 20 & 165 & 59 & 207 \\
\hline HSP.B2 & 1964.0 & 0.31 & 10 & 53 & 94 & 0.23 & 16 & 504 & 25 & 83 & 15 & 149 & 58 & 200 \\
\hline HSP.B2 & 1958.8 & 0.31 & 8.8 & 45 & 96 & 0.23 & 15 & 493 & 22 & 81 & 16 & 149 & 59 & 197 \\
\hline HSP.B2 & 1953.4 & 0.16 & 2.0 & 26 & 48 & 0.11 & 8.5 & 332 & 18 & 36 & 11 & 144 & 30 & 101 \\
\hline \multicolumn{15}{|c|}{ Lower Reservoir Site: } \\
\hline HSP.1 & 1997.6 & 1.3 & 17 & 37 & 52 & 0.23 & 16 & 692 & 13 & 41 & 11 & 168 & 60 & 155 \\
\hline HSP.1 & 1992.6 & 1.2 & 20 & 38 & 55 & 0.23 & 14 & 662 & 12 & 41 & 13 & 155 & 59 & 155 \\
\hline HSP.1 & 1989.1 & 1.2 & 16 & 40 & 57 & 0.20 & 19 & 636 & 11 & 53 & 12 & 168 & 57 & 153 \\
\hline HSP.1 & 1985.3 & 1.4 & 16 & 43 & 79 & 0.27 & 17 & 634 & 7.9 & 71 & 12 & 178 & 64 & 153 \\
\hline HSP.1 & 1980.7 & 1.7 & 16 & 50 & 108 & 0.30 & 14 & 544 & 14 & 70 & 11 & 171 & 68 & 159 \\
\hline HSP.1 & 1975.2 & 1.7 & 11 & 72 & 80 & 0.42 & 12 & 480 & 12 & 89 & 15 & 155 & 67 & 124 \\
\hline HSP.1 & 1968.8 & 1.8 & 6.1 & 72 & 83 & 0.40 & 11 & 486 & 11 & 114 & 12 & 161 & 59 & 132 \\
\hline HSP.1 Dup & 1968.8 & 1.9 & 6.5 & 74 & 83 & 0.42 & 12 & 484 & 12 & 114 & 12 & 166 & 63 & 128 \\
\hline HSP.1 & 1964.0 & 2.2 & 10 & 70 & 95 & 0.33 & 10 & 506 & 13 & 79 & 11 & 144 & 78 & 135 \\
\hline HSP.1 & 1955.6 & 0.15 & 5.1 & 11 & 11 & 0.17 & 1.0 & 371 & 6.0 & 42 & 5.6 & 135 & 36 & 38 \\
\hline HSP.1 & not assigned & 0.10 & 6.8 & 16 & 9.7 & 0.09 & 2.1 & 386 & 11 & 48 & 5.2 & 139 & 31 & 25 \\
\hline HSP.1 & not assigned & 0.14 & 9.8 & 17 & 8.1 & 0.12 & 2.1 & 384 & 5.8 & 32 & 6.4 & 125 & 34 & 30 \\
\hline HSP.1 Dup & not assigned & 0.12 & 8.6 & 14 & 8.6 & 0.11 & 3.3 & 396 & 5.3 & 37 & 5.6 & 129 & 34 & 29 \\
\hline HSP.1 & not assigned & 0.14 & 5.8 & 16 & 3.8 & 0.04 & 5.3 & 422 & 3.8 & 23 & 7.6 & 146 & 42 & 26 \\
\hline \multicolumn{15}{|c|}{ Mill Creek (Harris Pond inflow): } \\
\hline Mill Creek & $3 / 21$ to $3 / 27 / 0$ & 0.85 & 14 & 46 & 38 & na & 16 & 1,310 & 17 & 76 & 10 & 146 & 59 & 161 \\
\hline Mill Creek & $5 / 22$ to $5 / 24 / 0$ & 2.0 & 60 & 51 & 51 & na & 16 & 9,740 & 22 & 103 & 7.7 & 121 & 62 & 507 \\
\hline
\end{tabular}


Table A4. Selected chlorinated hydrocarbon compound concentrations in bottom and suspended sediments. All concentrations in ug/kg. Remark: <, non-detection at indicated value.

Deposition Date

Depth Min Depth Max Depth Mid (cores) or Sample

(cm)
Date (streams)

Technical

Core ID or Stream (cm) (cm)

(cm)

(streams)

Primary (Lower) Reservoir Site:

$\begin{array}{cc}\text { CMO.1 } & 0 \\ \text { CMO.1 } & 5 \\ \text { CMO.1 } & 10 \\ \text { CMO.1 } & 20 \\ \text { CMO.1 } & 30 \\ \text { CMO.1dup } & 30 \\ \text { CMO.1 } & 45 \\ \text { CMO.1dup } & 45 \\ \text { CMO.1 } & 60 \\ \text { CMO.1 } & 75 \\ \text { CMO.1 } & 90\end{array}$

Mid Reservoir Site: CMO.3

CMO.3

CMO.3

$5 \quad 2.5$

2000.8
1999.9
1998.7
1995.3
1990.6
1990.6
1982.5
1982.5
1974.1
1969.1
1958.6

Chlordane

Dieldrin

DDE

DDD

DDT

РСB 1242

Upper Reservoir Site:

$\begin{array}{cccc}\text { CMO.5 } & 0 & 10 & 5 \\ \text { CMO.5 } & 20 & 30 & 25 \\ \text { CMO.5 } & 40 & 50 & 45\end{array}$

Como Lake Inflow:

Lake Como Inflow

Lake Como Inflow

Lake Como Inflow

Lake Como Inflow

2.5
7.5
12.5
22.5
32.5
32.5
47.5
47.5
62.5
77.5
92.5

5
45
95

5
25
45

1958.6

1999

1970

1950

2000

1990

1965

150

150
130

130
130

160

160
330

330
290
185

290
185

185
180

180
120

600

150

12
2.7

2.7

1.4
1.6

$<\quad 0.8$

$<\quad 0.7$

1.8

22

2.2
1.4

$<\quad 0.8$

$<\quad 0.6$

$140 \quad 6.2$

$250<1.0$

$250<1.0$
$<\quad 5.0<0.5$

$6.3 \quad 2.6$

$2.6<1.5<15$

$7.3 \quad 6.0<1.5<15$

8.8

13

19

19
17

17

16
7.6

7.6
30

30
22

9.1

$0.6-6.6$

$<\quad 0.5$

$\begin{array}{lll}0.5 & 0.8 & 33 \\ <\quad 0.5 & 15 & 34\end{array}$

$0.5<0.5$
$6.0<0.9$

$6.0<0.5$
$65<1.1<57$

$\begin{array}{lll}83 & 1.2 & 45\end{array}$

Echo Lake

Upper Reservoir Site: 


\begin{tabular}{|c|c|c|c|c|c|c|c|c|c|c|c|c|c|c|c|c|}
\hline ECO.3 & 0 & 5 & 2.5 & 1999 & & 130 & & 5.1 & & 24 & & 9.8 & & 12 & $<$ & 10 \\
\hline ECO.3 & 25 & 30 & 27.5 & 1988 & & 410 & $<$ & 0.5 & & 39 & & 12 & & 3.4 & & 24 \\
\hline ECO. 3 & 50 & 55 & 52.5 & 1972 & & 220 & $<$ & 0.5 & & 68 & & 25 & & 1.4 & & 110 \\
\hline \multicolumn{17}{|c|}{ Middle Reservoir Site: } \\
\hline ECO. 4 & 0 & 8 & 4 & 1999 & & 99 & & 1.7 & & 20 & & 5.4 & & 2.3 & $<$ & 5.0 \\
\hline ECO.4 & 40 & 48 & 44 & 1972 & & 120 & $<$ & 0.5 & & 77 & & 18 & & 2.3 & & 64 \\
\hline ECO. 4 & 72 & 80 & 76 & 1952 & $<$ & 5.0 & $<$ & 0.5 & & 4.1 & & 13 & & 0.6 & $<$ & 5.0 \\
\hline \multicolumn{17}{|c|}{ Primary (Lower) Reservoir Site: } \\
\hline ECO. 1 & 0 & 5 & 2.5 & 1999.7 & & 130 & & 3.3 & & 20 & & 6.3 & & 3.2 & $<$ & 15 \\
\hline ECO.1 & 5 & 10 & 7.5 & 1998.3 & & 85 & & 0.7 & & 16 & & 4.7 & & 1.4 & $<$ & 10 \\
\hline ECO.1 & 10 & 15 & 12.5 & 1996.4 & & 85 & & 0.6 & & 25 & & 6.1 & & 1.2 & & 11 \\
\hline ECO.1 & 20 & 25 & 22.5 & 1991.7 & & 66 & $<$ & 0.5 & & 23 & & 7.8 & & 1.5 & & 11 \\
\hline ECO.1 & 30 & 35 & 32.5 & 1985.9 & & 100 & $<$ & 0.5 & & 33 & & 7.9 & & 1.0 & & 40 \\
\hline ECO.1 & 40 & 45 & 42.5 & 1980.6 & & 230 & $<$ & 0.5 & & 40 & & 20 & $<$ & 0.5 & & 29 \\
\hline ECO.1 & 55 & 60 & 57.5 & 1972.5 & & 33 & $<$ & 0.5 & & 85 & & 130 & & 2.9 & & 110 \\
\hline ECO.1 & 65 & 70 & 67.5 & 1966.8 & $<$ & 9.3 & $<$ & 0.5 & & 22 & & 30 & & 0.9 & & 48 \\
\hline ECO.1 & 75 & 80 & 77.5 & 1961.1 & $<$ & 5.0 & $<$ & 0.5 & & 25 & & 80 & & 1.2 & & 22 \\
\hline ECO. 1 & 90 & 97 & 93.5 & 1951.4 & $<$ & 5.0 & $<$ & 0.5 & & 6.0 & & 8.4 & & 0.6 & $<$ & 5.0 \\
\hline \multicolumn{17}{|l|}{ Echo Lake Inflow: } \\
\hline Echo Lake Inflow & & & & $05 / 28 / 01$ & & 79 & & 6.1 & & 6.8 & $<$ & 2.0 & & 10 & & 15 \\
\hline Echo Lake Inflow & & & & 08/17/01 & & 220 & & 10 & & 33 & & 5.2 & & 130 & & 30 \\
\hline Echo Lake Inflow & & & & 09/20/01 & & 260 & & 28 & & 19 & $<$ & 16 & & 42 & $<$ & 100 \\
\hline Echo Lake Inflow & & & & $10 / 11 / 01$ & & 220 & & 23 & & 30 & $<$ & 18 & & 78 & & 19 \\
\hline \multicolumn{17}{|c|}{ Fosdic Lake } \\
\hline \multicolumn{17}{|c|}{ Upper Reservoir Site: } \\
\hline FOS. 5 & 0 & 10 & 5 & 2000 & & 140 & & 5.1 & & 19 & $<$ & 6.1 & & 3.0 & $<$ & 10 \\
\hline FOS.5 & 40 & 50 & 45 & 1970 & & 120 & & 1.9 & & 55 & $<$ & 15 & & 1.3 & $<$ & 12 \\
\hline \multicolumn{17}{|c|}{ Primary (Mid) Reservoir Site: } \\
\hline FOS. 4 & 0 & 5 & 2.5 & 2000.4 & & 140 & & 9.0 & & 19 & $<$ & 8.3 & & 3.0 & $<$ & 15 \\
\hline FOS.4 & 5 & 10 & 7.5 & 1998.4 & & 150 & & 4.7 & & 25 & $<$ & 6.1 & & 3.4 & $<$ & 15 \\
\hline FOS.4 & 10 & 15 & 12.5 & 1996.2 & & 120 & & 3.3 & & 24 & $<$ & 7.4 & $<$ & 1.5 & $<$ & 10 \\
\hline FOS.4 & 20 & 25 & 22.5 & 1991.0 & & 98 & & 1.7 & & 21 & $<$ & 3.3 & $<$ & 1.0 & $<$ & 15 \\
\hline FOS. 4 & 30 & 35 & 32.5 & 1983.3 & & 68 & & 1.8 & & 38 & $<$ & 7.6 & & 1.5 & $<$ & 10 \\
\hline FOS.4 & 45 & 50 & 47.5 & 1968.9 & & 47 & & 1.6 & & 67 & & 8.8 & & 1.4 & $<$ & 30 \\
\hline FOS.4 & 55 & 60 & 57.5 & 1959.8 & & 19 & & 0.5 & & 48 & & 23 & & 0.6 & $<$ & 14 \\
\hline FOS.4 & 70 & 75 & 72.5 & 1944.6 & $<$ & 5.0 & $<$ & 0.5 & & 13 & & 17 & $<$ & 0.5 & $<$ & 9.3 \\
\hline FOS.4 & 85 & 90 & 87.5 & 1928.3 & $<$ & 5.0 & $<$ & 0.5 & & 0.4 & & 0.5 & $<$ & 0.5 & $<$ & 5.0 \\
\hline FOS. 4 & 100 & 105 & 102.5 & 1912.6 & $<$ & 5.0 & $<$ & 0.5 & $<$ & 0.5 & $<$ & 0.5 & $<$ & 0.5 & $<$ & 5.0 \\
\hline \multicolumn{17}{|c|}{ Lower Reservoir Site: } \\
\hline FOS. 2 & 0 & 10 & 5 & 2000 & & 170 & & 6.5 & & 21 & $<$ & 6.1 & $<$ & 0.5 & $<$ & 15 \\
\hline FOS.2 & 50 & 60 & 55 & 1965 & & 31 & & 1.0 & & 56 & & 33 & & 1.7 & $<$ & 21 \\
\hline
\end{tabular}


FOS.2

Fosdic Lake Inflow

Fosdic Lake Inflow

Fosdic Lake Inflow

Fosdic Lake Inflow

Fosdic Lake Inflow

Upper Mystic Lake

Upper Lake Site:

MYS.B2

MYS.B2

MYS.B2

MYS.B2

MYS.B2

MYS.B2

MYS.B2

MYS.B2

MYS.B2

MYS.B2

MYS.B2

MYS.B2

Lower Lake Site:

MYS.2

MYS.2

MYS.2

Aberjona River (Upper Mystic Lake inflow)

Aberjona River

Aberjona River

Aberjona River

Harris Pond

Upper Reservoir Site:

HSP.B3 0-6

HSP.B3 24-30

Middle Reservoir Site:

HSP.B2 0-2

HSP.B2 2-4

HSP.B2 4-6

HSP B2 6-8

HSP.B2 8-10

HSP.B2 10-12

HSP.B2 12-14 $\begin{array}{lll}100 & 110 & 105\end{array}$

$08 / 11 / 01$

09/18/01

$10 / 10 / 01$

$12 / 06 / 01$

2000.7

2000.7

2000.7

1999.0

1996.5

1989.1

1982.7

1975.2

1967.9

1958

1958

1943

4

16.5

27.5

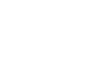

$3 / 21$ to $3 / 22 / 01$

$3 / 22$ to $3 / 23 / 01$

$8 / 29$ to $8 / 30 / 02$

1998
1968
2000
1997.9
1995.7
1993.3
1990.7
1987.6
1984.4

1100
60
120
660

$\begin{array}{ccccc}420 & < & 6.8 & 40 & 4 \\ 360 & < & 4.8 & 37 & 37 \\ 320 & < & 5.6 & 31 & 3 \\ 340 & < & 3.2 & 36 & 37 \\ 320 & < & 2.3 & 29 & 33 \\ 660 & < & 2.5 & 77 & 63 \\ 710 & < & 2.5 & 81 & 66 \\ 790 & < & 3.0 & 140 & 140 \\ 510 & < & 3.0 & 300 & 280 \\ 280 & < & 2.5 & 270 & 2 \\ 270 & < & 2.5 & 250 & 2 \\ 62 & < & 2.0 & 56 & 34 \\ & & & & \\ 450 & < & 2.0 & 47 & 52 \\ 420 & < & 1.5 & 83 & 49 \\ 550 & < & 2.0 & 180 & 1 \\ & & & & 160 \\ 390 & & 5.4 & 28 & 33 \\ 410 & & 7.7 & 56 & 35 \\ 490 & < & 20 & 39 & \end{array}$

\begin{tabular}{|c|c|c|}
\hline 44 & 24 & \\
\hline 37 & 19 & \\
\hline 34 & 17 & \\
\hline 37 & 19 & \\
\hline 33 & 14 & \\
\hline 63 & 32 & \\
\hline 66 & 34 & \\
\hline 140 & 88 & \\
\hline 280 & 100 & \\
\hline 240 & 92 & \\
\hline 200 & 90 & \\
\hline 34 & 16 & \\
\hline 52 & 33 & \\
\hline 49 & 26 & \\
\hline 140 & 35 & \\
\hline 16 & 53 & \\
\hline 33 & 77 & \\
\hline 35 & 59 & $<$ \\
\hline
\end{tabular}

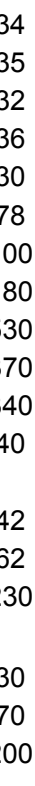

$\begin{array}{lll}27 & < & 2.5 \\ 32 & < & 1.5 \\ 22 & < & 4 \\ 26 & < & 2 \\ 25 & < & 2.5 \\ 26 & < & 2.0 \\ 32 & < & 2.5 \\ 23 & < & 2.0 \\ 33 & < & 2.0\end{array}$

\begin{tabular}{|c|c|c|c|c|c|}
\hline 12 & $<$ & 2.5 & $<$ & 2.5 & \\
\hline 15 & $<$ & 1.5 & $<$ & 1.5 & \\
\hline 20 & & 14 & $<$ & 4 & $<$ \\
\hline 18 & & 13 & & 5.0 & $<$ \\
\hline 13 & $<$ & 2.5 & $<$ & 2.5 & \\
\hline 13 & $<$ & 2.0 & $<$ & 2.0 & \\
\hline 16 & $<$ & 2.5 & $<$ & 2.5 & \\
\hline 24 & & 16 & & 5.3 & $<$ \\
\hline 18 & $<$ & 2.0 & & 2 & \\
\hline
\end{tabular}




\begin{tabular}{|c|c|c|c|c|c|c|c|c|c|c|c|c|c|c|c|}
\hline HSP.B2 14-16 & 14.0 & 16.0 & 15.0 & 1980.6 & & 36 & $<$ & 2.5 & 17 & $<$ & 2.5 & $<$ & 2.5 & & 8 \\
\hline HSP.B2 14-16 dup & 14.0 & 16.0 & 15.0 & 1980.6 & & 32 & $<$ & 2.5 & 18 & $<$ & 2.5 & $<$ & 2.5 & & 9 \\
\hline HSP.B2 16-18 & 16.0 & 18.0 & 17.0 & 1976.7 & & 40 & $<$ & 3.0 & 24 & $<$ & 3.0 & $<$ & 3.0 & & 12 \\
\hline HSP.B2 18-20 & 18.0 & 20.0 & 19.0 & 1973.1 & & 36 & $<$ & 2.5 & 20 & $<$ & 2.5 & $<$ & 2.5 & & 10 \\
\hline HSP.B2 20-22 & 20.0 & 22.0 & 21.0 & 1969.2 & & 26 & $<$ & 3 & 22 & & 19 & $<$ & 4 & $<$ & 30 \\
\hline HSP.B2 22-24 & 22.0 & 24.0 & 23.0 & 1965.1 & & 41 & $<$ & 2.0 & 25 & $<$ & 2.0 & & 3 & & 13 \\
\hline \multicolumn{16}{|c|}{ Lower Reservoir Site: } \\
\hline HSP.1 0-6 & 0 & 6 & 3.0 & 1997 & & 47 & & 5 & 18 & $<$ & 3.5 & $<$ & 3.5 & & 9 \\
\hline HSP.1 18-24 & 18 & 24 & 21.0 & 1966 & & 57 & $<$ & 2.5 & 44 & $<$ & 2.5 & $<$ & 2.5 & & 42 \\
\hline \multicolumn{16}{|c|}{ Mill Creek (Harris Pond inflow): } \\
\hline Mill Creek & & & & $3 / 21$ to $3 / 27 / 01$ & $<$ & 610 & $<$ & 61 & 35 & $<$ & 61 & $<$ & 61 & $<$ & 610 \\
\hline Mill Creek & & & & $5 / 22$ to $5 / 24 / 01$ & $<$ & 110 & $<$ & 11 & 30 & & 12 & & 23 & $<$ & 110 \\
\hline
\end{tabular}


Calculated Values

(nondetections treated as

$$
\text { zero) }
$$

\begin{tabular}{|c|c|c|c|c|c|c|c|c|c|}
\hline Core ID or Stream & Date & & РСВ 1254 & & РCB 1260 & & $\begin{array}{c}\text { TOTAL } \\
\text { PCB } \\
\end{array}$ & $\begin{array}{c}\text { TOTAL } \\
\text { DDT }\end{array}$ & Remarks \\
\hline \multicolumn{10}{|c|}{ Como Lake } \\
\hline \multicolumn{10}{|c|}{ Primary (Lower) Reservoir Site: } \\
\hline CMO.1 & 2000.8 & & 18 & & 19 & & 37 & 8.9 & \\
\hline CMO.1 & 1999.9 & & 21 & & 20 & & 41 & 13 & \\
\hline CMO.1 & 1998.7 & & 25 & & 27 & & 59 & 18 & \\
\hline CMO.1 & 1995.3 & & 46 & & 43 & & 100 & 27 & \\
\hline СMO.1 & 1990.6 & & 77 & & 63 & & 170 & 55 & \\
\hline CMO.1dup & 1990.6 & & 74 & & 58 & & 150 & 56 & \\
\hline СMO.1 & 1982.5 & & 57 & & 26 & & 120 & 18 & \\
\hline CMO.1dup & 1982.5 & & 54 & & 23 & & 110 & 18 & \\
\hline CMO.1 & 1974.1 & & 26 & & 18 & & 50 & 14 & \\
\hline CMO.1 & 1969.1 & & 120 & & 68 & & 240 & 95 & \\
\hline CMO.1 & 1958.6 & & 110 & & 300 & & 460 & 106 & \\
\hline \multicolumn{10}{|l|}{ Mid Reservoir Site: } \\
\hline CMO.3 & 1999 & & 23 & & 23 & & 46 & 12 & \\
\hline CMO.3 & 1970 & & 78 & & 64 & & 160 & 36 & \\
\hline CMO.3 & 1950 & $<$ & 5.0 & $<$ & 5.0 & $<$ & 0 & 0.6 & \\
\hline \multicolumn{10}{|c|}{ Upper Reservoir Site: } \\
\hline CMO.5 & 2000 & & 25 & & 27 & & 60 & 15 & \\
\hline CMO.5 & 1990 & & 26 & & 27 & & 62 & 38 & \\
\hline CMO.5 & 1965 & & 530 & & 250 & & 780 & 96 & \\
\hline \multicolumn{10}{|l|}{ Como Lake Inflow: } \\
\hline Lake Como Inflow & 08/30/01 & & 75 & & 70 & & 230 & 18 & \\
\hline Lake Como Inflow & 09/20/01 & & 79 & & 73 & & 290 & 16 & \\
\hline Lake Como Inflow & $11 / 09 / 01$ & $<$ & 670 & $<$ & 670 & $<$ & 0 & 22 & \\
\hline Lake Como Inflow & $01 / 23-24 / 02$ & & 15 & & 16 & & 50 & 4.3 & \\
\hline
\end{tabular}




\begin{tabular}{|c|c|c|c|c|c|c|c|c|c|}
\hline ECO.3 & 1999 & & 71 & & 43 & & 110 & & 46 \\
\hline ECO.3 & 1988 & & 60 & & 62 & & 150 & & 54 \\
\hline ECO.3 & 1972 & & 290 & & 300 & & 700 & & 94 \\
\hline \multicolumn{10}{|c|}{ Middle Reservoir Site: } \\
\hline ECO. 4 & 1999 & & 92 & & 44 & & 140 & & 28 \\
\hline ECO.4 & 1972 & & 170 & & 130 & & 360 & & 97 \\
\hline ECO.4 & 1952 & & 15 & & 8.0 & & 23 & & 18 \\
\hline \multicolumn{10}{|c|}{ Primary (Lower) Reservoir Site: } \\
\hline ECO.1 & 1999.7 & & 110 & & 46 & & 160 & & 30 \\
\hline ECO.1 & 1998.3 & & 150 & & 49 & & 200 & & 22 \\
\hline ECO.1 & 1996.4 & & 470 & & 88 & & 570 & & 32 \\
\hline ECO.1 & 1991.7 & & 33 & & 35 & & 79 & & 32 \\
\hline ECO.1 & 1985.9 & & 58 & & 45 & & 140 & & 42 \\
\hline ECO.1 & 1980.6 & & 75 & & 65 & & 170 & & 60 \\
\hline ECO.1 & 1972.5 & & 210 & & 170 & & 490 & & 218 \\
\hline ECO.1 & 1966.8 & & 91 & & 102 & & 240 & & 53 \\
\hline ECO.1 & 1961.1 & & 110 & & 170 & & 300 & & 106 \\
\hline ECO.1 & 1951.4 & & 14 & & 24 & & 38 & & 15 \\
\hline \multicolumn{10}{|l|}{ Echo Lake Inflow: } \\
\hline Echo Lake Inflow & 05/28/01 & & 25 & & 20 & & 60 & & 17 \\
\hline Echo Lake Inflow & 08/17/01 & & 110 & & 59 & & 200 & & 168 \\
\hline Echo Lake Inflow & 09/20/01 & & 54 & & 60 & & 110 & & 61 \\
\hline Echo Lake Inflow & 10/11/01 & & 66 & & 57 & & 140 & & 108 \\
\hline \multicolumn{10}{|c|}{ Fosdic Lake } \\
\hline \multicolumn{10}{|c|}{ Upper Reservoir Site: } \\
\hline FOS. 5 & 2000 & & 37 & & 52 & & 89 & & 22 \\
\hline FOS.5 & 1970 & & 130 & & 120 & & 250 & & 56 \\
\hline \multicolumn{10}{|c|}{ Primary (Mid) Reservoir Site: } \\
\hline FOS. 4 & 2000.4 & & 40 & & 59 & & 99 & & 22 \\
\hline FOS.4 & 1998.4 & & 51 & & 70 & & 120 & & 28 \\
\hline FOS.4 & 1996.2 & & 48 & & 72 & & 120 & & 24 \\
\hline FOS.4 & 1991.0 & & 38 & & 54 & & 92 & & 21 \\
\hline FOS.4 & 1983.3 & & 100 & & 110 & & 210 & & 40 \\
\hline FOS.4 & 1968.9 & & 170 & & 160 & & 330 & & 77 \\
\hline FOS.4 & 1959.8 & & 56 & & 87 & & 140 & & 72 \\
\hline FOS.4 & 1944.6 & & 13.4 & & 17 & & 30 & & 30 \\
\hline FOS.4 & 1928.3 & $<$ & 5.0 & $<$ & 5.0 & $<$ & 15 & & 0.9 \\
\hline FOS.4 & 1912.6 & $<$ & 5.0 & $<$ & 5.0 & $<$ & 15 & $<$ & 1.5 \\
\hline \multicolumn{10}{|c|}{ Lower Reservoir Site: } \\
\hline FOS. 2 & 2000 & & 48 & & 66 & & 110 & & 21 \\
\hline FOS.2 & 1965 & & 92 & & 94 & & 190 & & 91 \\
\hline
\end{tabular}




\begin{tabular}{|c|c|c|c|c|c|c|c|c|c|c|}
\hline FOS. 2 & 1920 & $<$ & 5.0 & $<$ & 5.0 & $<$ & 15 & $<$ & 1.5 & \\
\hline \multicolumn{11}{|l|}{ Fosdic Lake Inflow } \\
\hline Fosdic Lake Inflow & 08/11/01 & $<$ & 1100 & $<$ & 1100 & $<$ & 3300 & $<$ & 330 & \\
\hline Fosdic Lake Inflow & 09/18/01 & $<$ & 60 & $<$ & 60 & $<$ & 180 & & 30 & \\
\hline Fosdic Lake Inflow & $10 / 10 / 01$ & $<$ & 120 & $<$ & 120 & $<$ & 360 & & 14080 & \\
\hline Fosdic Lake Inflow & $12 / 06 / 01$ & $<$ & 660 & $<$ & 660 & $<$ & 2000 & & 20 & \\
\hline \multicolumn{11}{|l|}{ Upper Mystic Lake } \\
\hline \multicolumn{11}{|l|}{ Upper Lake Site: } \\
\hline MYS.B2 & 2000.7 & & 110 & & 91 & & 240 & & 108 & \multirow{16}{*}{$\begin{array}{l}0-6 \mathrm{~cm} \text { mixing zone in core (based on } 210 \mathrm{~Pb} \text { ) } \\
\text { assigned sampling date assuming } \\
\text { instantaneous mixing. }\end{array}$} \\
\hline MYS.B2 & 2000.7 & & 100 & & 85 & & 220 & & 93 & \\
\hline MYS.B2 & 2000.7 & & 87 & & 71 & & 190 & & 82 & \\
\hline MYS.B2 & 1999.0 & & 100 & & 80 & & 220 & & 92 & \\
\hline MYS.B2 & 1996.5 & & 89 & & 69 & & 190 & & 76 & \\
\hline MYS.B2 & 1989.1 & & 370 & & 230 & & 680 & & 172 & \\
\hline MYS.B2 & 1982.7 & & 420 & & 250 & & 770 & & 181 & \\
\hline MYS.B2 & 1975.2 & & 550 & & 320 & & 1000 & & 368 & \\
\hline MYS.B2 & 1967.9 & & 950 & & 490 & & 2000 & & 680 & \\
\hline MYS.B2 & 1958 & & 830 & & 490 & & 1700 & & 602 & \\
\hline MYS.B2 & 1958 & & 780 & & 450 & & 1600 & & 540 & \\
\hline MYS.B2 & 1943 & & 110 & & 180 & & 330 & & 106 & \\
\hline \multicolumn{10}{|l|}{ Lower Lake Site: } & \\
\hline MYS.2 & & & 150 & & 120 & & 310 & & 132 & \\
\hline MYS.2 & & & 250 & & 200 & & 510 & & 158 & \\
\hline MYS.2 & & & 800 & & 370 & & 1400 & & 355 & \\
\hline \multicolumn{11}{|c|}{ Aberjona River (Upper Mystic Lake inflow) } \\
\hline Aberjona River & $3 / 21$ to $3 / 22 / 01$ & & 87 & & 60 & & 180 & & 97 & \multirow{3}{*}{$\begin{array}{l}\text { Mean of these two samples over 3-day event } \\
\text { used to represent event. }\end{array}$} \\
\hline Aberjona River & $3 / 22$ to $3 / 23 / 01$ & & 180 & & 140 & & 390 & & 166 & \\
\hline Aberjona River & $8 / 29$ to $8 / 30 / 02$ & & 150 & & 170 & & 320 & & 133 & \\
\hline \multicolumn{11}{|c|}{ Harris Pond } \\
\hline \multicolumn{11}{|c|}{ Upper Reservoir Site: } \\
\hline HSP.B3 0-6 & 1998 & & 36 & & 62 & & 100 & & 12 & \\
\hline HSP.B3 24-30 & 1968 & & 52 & & 87 & & 150 & & 15 & \\
\hline \multicolumn{11}{|c|}{ Middle Reservoir Site: } \\
\hline HSP.B2 0-2 & 2000 & & 57 & & 111 & & 170 & & 34 & \\
\hline HSP.B2 2-4 & 1997.9 & & 58 & & 100 & & 160 & & 36 & \\
\hline HSP.B2 4-6 & 1995.7 & & 39 & & 70 & & 120 & & 13 & \\
\hline HSP.B2 6-8 & 1993.3 & & 39 & & 68 & & 110 & & 13 & \\
\hline HSP.B2 8-10 & 1990.7 & & 48 & & 83 & & 140 & & 16 & \\
\hline HSP.B2 10-12 & 1987.6 & & 82 & & 160 & & 240 & & 45 & \\
\hline HSP.B2 12-14 & 1984.4 & & 50 & & 88 & & 150 & & 20 & \\
\hline
\end{tabular}




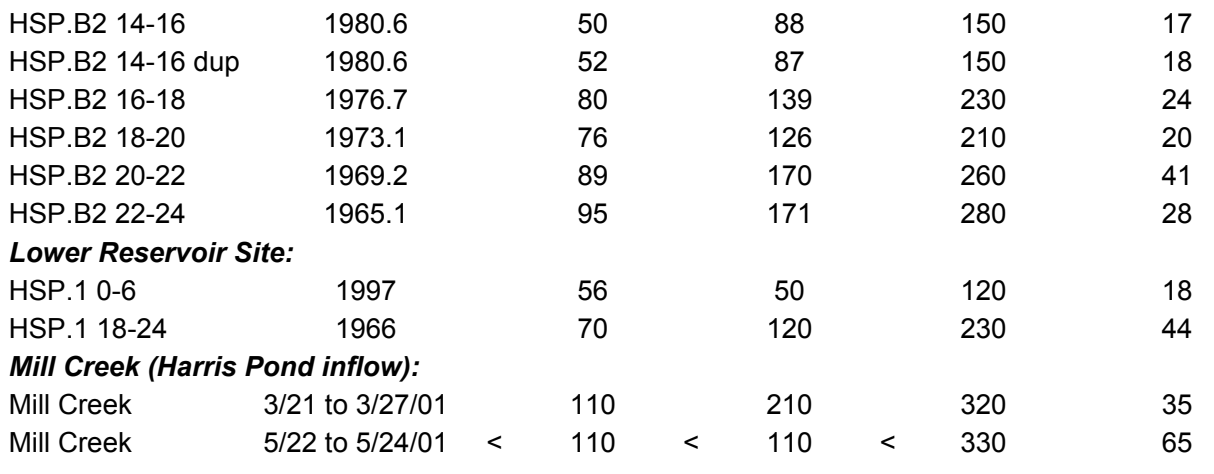


Table A5. Selected polycyclic aromatic hydrocarbon compound concentrations in bottom and suspended sediment samples. All concentrations in ug/kg.

Deposition

Date (cores) or

\begin{tabular}{|c|c|c|c|c|c|c|c|c|c|c|c|c|c|}
\hline $\begin{array}{c}\text { Core ID or } \\
\text { Stream }\end{array}$ & $\begin{array}{c}\text { Depth Min } \\
(\mathbf{c m})\end{array}$ & $\begin{array}{l}\text { Depth Max } \\
\text { (cm) }\end{array}$ & $\begin{array}{l}\text { Depth Mid } \\
\text { (cm) }\end{array}$ & $\begin{array}{l}\text { Date (cores) or } \\
\text { Sample Date } \\
\text { (streams) }\end{array}$ & $\begin{array}{l}\text { Naphtha- } \\
\text { lene }\end{array}$ & $\begin{array}{c}9 \mathrm{H}- \\
\text { Fluorene }\end{array}$ & $\begin{array}{c}\text { Phenan- } \\
\text { threne }\end{array}$ & $\begin{array}{c}\text { Anthrac- } \\
\text { ene }\end{array}$ & $\begin{array}{c}\text { Fluoran- } \\
\text { thene }\end{array}$ & Pyrene & $\begin{array}{c}\text { Benz (a) } \\
\text { Anthra- } \\
\text { cene }\end{array}$ & Chrysene & $\begin{array}{c}\text { Benzo (a) } \\
\text { pyrene }\end{array}$ \\
\hline \multicolumn{14}{|l|}{ Como Lake } \\
\hline \multicolumn{14}{|c|}{ Upper Reservoir Site: } \\
\hline CMO.5 & 0 & 10 & 5 & 2000 & 33 & 93 & 1,360 & 196 & 4,460 & 2,530 & 1,710 & 2,560 & 2,020 \\
\hline CMO.5 & 20 & 30 & 25 & 1990 & 19 & 85 & 1,240 & 191 & 4,260 & 3,360 & 1,740 & 2,360 & 1,990 \\
\hline CMO.5 & 40 & 50 & 45 & 1965 & 19 & 44 & 372 & 83 & 640 & 474 & 288 & 282 & 267 \\
\hline \multicolumn{14}{|c|}{ Mid Reservoir Site: } \\
\hline CMO. 3 & 0 & 10 & 5 & 1999 & 27 & 78 & 798 & 140 & 2,000 & 1,420 & 948 & 1,510 & 1,140 \\
\hline CMO.3 & 40 & 50 & 45 & 1970 & 14 & 59 & 402 & 106 & 710 & 561 & 333 & 502 & 371 \\
\hline CMO.3 & 90 & 100 & 95 & 1950 & 4 & 15 & 38 & 20 & 61 & 59 & 44 & 76 & 43 \\
\hline \multicolumn{14}{|c|}{ Primary (Lower Reservoir) Site: } \\
\hline CMO.1 & 0 & 5 & 2.5 & 2000.8 & 19 & 37 & 417 & 77 & 1,080 & 814 & 426 & 884 & 564 \\
\hline CMO.1 & 5 & 10 & 7.5 & 1999.9 & 27 & 96 & 1,080 & 239 & 2,660 & 1,920 & 1,300 & 2,030 & 1,460 \\
\hline CMO.1 & 10 & 15 & 12.5 & 1998.7 & 22 & 52 & 611 & 120 & 1,680 & 1,260 & 977 & 1,500 & 1,030 \\
\hline CMO.1 & 20 & 25 & 22.5 & 1995.3 & 10 & 31 & 290 & 81 & 751 & 576 & 350 & 610 & 439 \\
\hline СMO.1 & 30 & 35 & 32.5 & 1990.6 & 18 & 58 & 537 & 131 & 1,180 & 938 & 754 & 889 & 629 \\
\hline CMO.1 dup & 30 & 35 & 32.5 & 1990.6 & 17 & 63 & 484 & 134 & 1,020 & 802 & 495 & 731 & 550 \\
\hline СMO.1 & 45 & 50 & 47.5 & 1982.5 & 21 & 71 & 745 & 188 & 2,020 & 1,540 & 730 & 1,120 & 800 \\
\hline CMO.1 dup & 45 & 50 & 47.5 & 1982.5 & 22 & 70 & 715 & 183 & 1,900 & 1,430 & 701 & 1,070 & 798 \\
\hline СMO.1 & 60 & 65 & 62.5 & 1974.1 & 7 & 45 & 402 & 115 & 873 & 619 & 513 & 659 & 466 \\
\hline CMO.1 & 75 & 80 & 77.5 & 1969.1 & 12 & 102 & 949 & 265 & 1,240 & 965 & 647 & 808 & 577 \\
\hline СMO.1 & 90 & 95 & 92.5 & 1958.6 & 11 & 27 & 124 & 31 & 192 & 179 & 94 & 193 & 105 \\
\hline \multicolumn{14}{|c|}{ Como Lake Inflow: } \\
\hline \multicolumn{3}{|c|}{ Lake Como Inflow } & & 08/30/01 & 169 & 156 & 2,970 & 541 & 10,400 & 8,250 & 3,640 & 6,730 & 5,120 \\
\hline \multicolumn{3}{|c|}{ Lake Como Inflow } & & 09/20/01 & 213 & 179 & 2,740 & 488 & 9,190 & 7,360 & 2,560 & 6,210 & 4,010 \\
\hline \multicolumn{3}{|c|}{ Lake Como Inflow } & & 11/09/01 & 189 & 163 & 2,340 & 387 & 7,660 & 6,060 & 2,340 & 4,930 & 3,510 \\
\hline \multicolumn{3}{|c|}{ Lake Como Inflow } & & $01 / 23-24 / 02$ & 78 & 82 & 1,800 & 238 & 5,630 & 4,310 & 1,760 & 3,340 & 2,620 \\
\hline
\end{tabular}

Echo Lake 
Upper Reservoir Site:

$\begin{array}{cc}\text { ECO.3 } & \\ \text { ECO.3 } & 25 \\ \text { ECO.3 } & 50 \\ \text { ECO.3 Dup } & 50\end{array}$

0
25
50
50

Middle Reservoir Site:

$\begin{array}{cc}\text { ECO. } 4 & 0 \\ \text { ECO. } 4 & 40 \\ \text { ECO } 4 & 72 \\ \text { ECO } 4 \text { dup } & 72\end{array}$

ECO.4 dup 72

Primary (Lower) Reservoir Site:

$\begin{array}{lc}\text { ECO.1 } & 0 \\ \text { ECO. } 1 & 5 \\ \text { ECO. } 1 & 10 \\ \text { ECO. } 1 & 20 \\ \text { ECO.1 } & 30 \\ \text { ECO. } 1 & 40 \\ \text { ECO. } 1 & 55 \\ \text { ECO.1 } & 65 \\ \text { ECO. } 1 & 75 \\ \text { ECO.1 } & 90\end{array}$

ECO 1

2.5

27.5

1999

1988

1972

1972

51
64
69
51

1,020
965

230

3,280

52.5

51

752

145

2,440

1,120

2,490
1,930

$2,420 \quad 1,520$

132

1,190

904

984

1,500

1,060

1999

1972

1952

1952

28
25
10
9

$\begin{array}{ll}59 & 487 \\ 32 & 220\end{array}$

145

1,520

$961 \quad 571$

79

534
564

220

465

1,200

433
262

673

252
165

$\begin{array}{cc}1,280 & 794 \\ 390 & 276\end{array}$

48
48

290

257

165

185

162

$\begin{array}{llll}1999.7 & 25 & 39 & 308\end{array}$

1998.3

1996.4

1991.7

1985.9

1980.6

1972.5

1966.8

1961.1

1951.4

19
16
13
17
15
18
11
18
8

150
94
90
71
128
107
75
44
38
36

1,030

793

832

610

435

742
483

719

352

355

236

198

240

560
384

238

$581 \quad 401$

$334 \quad 197$

343

229
205

208

120
98

213

05/28/01

Echo Lake Inflow

Echo Lake Inflow

Echo Lake Inflow

Echo Lake Inflow

8
68
150
118

$\begin{array}{cc}22 & 63 \\ 111 & 1,820 \\ 105 & 1,880 \\ 128 & 2,020\end{array}$

52
387
342
407

801

6,310

92

09/20/01

1,880

8,130

4,650
4,570

4,570

$<440$

2,300
1,830

2,880

$\begin{array}{ll}972 & 535 \\ 695 & 385 \\ 642 & 357 \\ 387 & 257 \\ 539 & 394 \\ 312 & 212 \\ 350 & 242 \\ 160 & 121 \\ 177 & 102 \\ 159 & 150\end{array}$

Fosdic Lake

Upper Reservoir Site:

FOS 5$$
\text { FOS.5 }
$$

10

5

5
45

2000

1965

22
15

21
22
24
20
13
9

65
39

34
44
66
57
48
26

1,090
289
434
675
1,0
4
34

1,090
289


434
675
1,010
464
349
156

212
78


120
162
196
106
84
42

1,510
2,430
3,200
1,710
1,050
410

1,190
1,920
2,500
1,240
791
336

$\begin{array}{ccc}487 & 851 & 799 \\ 766 & 1,280 & 1,280 \\ 936 & 1,510 & 1,530 \\ 601 & 797 & 670 \\ 314 & 436 & 396 \\ 126 & 192 & 180\end{array}$




\begin{tabular}{|c|c|c|c|c|c|c|c|c|c|c|c|c|c|}
\hline FOS.4.dup & 45 & 50 & 47.5 & 1968.9 & 8 & 28 & 148 & 39 & 408 & 352 & 123 & 196 & 169 \\
\hline FOS.4 & 55 & 60 & 57.5 & 1959.8 & 6 & 17 & 88 & 31 & 257 & 240 & 85 & 145 & 121 \\
\hline FOS.4 & 70 & 75 & 72.5 & 1944.6 & 3 & 8 & 31 & 11 & 86 & 77 & 40 & 45 & 38 \\
\hline FOS.4 & 85 & 90 & 87.5 & 1928.3 & 4 & 14 & 15 & 8 & 41 & 40 & 16 & 21 & 19 \\
\hline FOS.4 & 100 & 105 & 102.5 & 1912.6 & 5 & 10 & 17 & 6 & 40 & 31 & 12 & 15 & 15 \\
\hline \multicolumn{14}{|c|}{ Lower Reservoir Site: } \\
\hline FOS. 2 & 0 & 10 & 5 & 2000 & 37 & 82 & 882 & 208 & 2,850 & 2,180 & 806 & 1,510 & 1,290 \\
\hline FOS.2 & 50 & 60 & 55 & 1965 & 6 & 17 & 73 & 27 & 315 & 295 & 69 & 128 & 110 \\
\hline FOS.2 & 100 & 110 & 105 & 1920 & 4 & 11 & 14 & 9 & 36 & 39 & 13 & 23 & 16 \\
\hline FOS.2 dup & 100 & 110 & 105 & 1920 & 5 & 12 & 15 & 10 & 39 & 43 & 14 & 25 & 19 \\
\hline \multicolumn{14}{|c|}{ Fosdic Lake Inflow } \\
\hline Fosdic Lake In & & & & 08/11/01 & 68 & 139 & 478 & 256 & 1,080 & 954 & 389 & 614 & 722 \\
\hline Fosdic Lake In & & & & 09/18/01 & 12 & 33 & 394 & 88 & 1,130 & 933 & 416 & 761 & 600 \\
\hline Fosdic Lake In & & & & $10 / 10 / 01$ & 48 & 52 & 609 & 134 & 1,730 & 1,300 & 496 & 1,100 & 775 \\
\hline Fosdic Lake In & & & & $12 / 06 / 01$ & 45 & 159 & 955 & 274 & 3,010 & 2,350 & 1,100 & 1,880 & 1,710 \\
\hline \multicolumn{14}{|c|}{ Upper Mystic Lake } \\
\hline \multicolumn{14}{|c|}{ Upper Lake Site: } \\
\hline MYS.B2 & 0 & 2 & 1 & 2000.7 & 286 & 334 & 5,290 & 1,050 & 15,400 & 12,200 & 5,730 & 9,200 & 7,660 \\
\hline MYS.B2 & 2 & 4 & 3 & 2000.7 & 147 & 206 & 3,060 & 567 & 8,590 & 6,970 & 3,210 & 5,140 & 4,240 \\
\hline MYS.B2 & 4 & 6 & 5 & 2000.7 & 292 & 382 & 5,070 & 898 & 13,800 & 11,000 & 4,710 & 7,660 & 6,240 \\
\hline MYS.B2 & 6 & 8 & 7 & 1999.0 & 342 & 427 & 5,610 & 1,000 & 15,200 & 12,200 & 5,060 & 8,030 & 6,350 \\
\hline MYS.B2 & 8 & 10 & 9 & 1996.5 & 274 & 369 & 4,300 & 842 & 12,200 & 10,000 & 4,200 & 6,870 & 5,350 \\
\hline MYS.B2 & 12 & 14 & 13 & 1989.1 & 224 & 380 & 3,710 & 1,000 & 13,500 & 11,300 & 5,660 & 8,010 & 7,390 \\
\hline MYS.B2 & 16 & 18 & 17 & 1982.7 & 249 & 301 & 3,110 & 1,040 & 10,700 & 9,450 & 4,760 & 6,450 & 6,420 \\
\hline MYS.B2 & 20 & 22 & 21 & 1975.2 & 395 & 338 & 3,220 & 1,740 & 11,100 & 10,500 & 4,560 & 6,800 & 7,280 \\
\hline MYS.B2 & 24 & 26 & 25 & 1967.9 & 531 & 384 & 3,410 & 2,160 & 11,500 & 10,900 & 4,820 & 6,900 & 7,180 \\
\hline MYS.B2 & 28 & 30 & 29 & 1958 & 530 & 452 & 3,800 & 2,130 & 10,800 & 10,100 & 4,630 & 6,460 & 6,770 \\
\hline MYS.B2 & 28 & 30 & 29 & 1958 & 509 & 366 & 3,640 & 2,070 & 9,840 & 9,460 & 4,370 & 5,910 & 6,350 \\
\hline MYS.B2 & 34 & 36 & 35 & 1943 & 399 & 501 & 4,200 & 1,390 & 12,200 & 10,800 & 5,550 & 7,150 & 6,560 \\
\hline \multicolumn{14}{|c|}{ Lower Lake Site: } \\
\hline MYS.2 & 0 & 8 & 4 & & 330 & 427 & 5,070 & 1,120 & 16,000 & 13,100 & 6,910 & 10,500 & 8,580 \\
\hline MYS.2 & 14 & 19 & 16.5 & & 335 & 387 & 3,540 & 1,050 & 12,200 & 10,800 & 5,500 & 7,950 & 6,420 \\
\hline MYS.2 & 25 & 30 & 27.5 & & 451 & 454 & 3,550 & 1,170 & 10,700 & 10,100 & 5,210 & 6,930 & 5,920 \\
\hline \multicolumn{14}{|c|}{ Aberjona River (Upper Mystic Lake inflow) } \\
\hline Aberjona River & & & & $3 / 21$ to $3 / 22 / 01$ & 143 & 411 & 7,980 & 1,410 & 17,600 & 12,400 & 6,410 & 8,240 & 8,010 \\
\hline Aberjona River & & & & $3 / 22$ to $3 / 23 / 01$ & 213 & 415 & 7,210 & 1,780 & 18,300 & 13,200 & 7,200 & 8,990 & 8,850 \\
\hline Aberjona River & & & & $8 / 29$ to $8 / 30 / 02$ & 299 & 340 & 5,440 & 1,810 & 16,700 & 12,600 & 6,780 & 10,100 & 8,940 \\
\hline
\end{tabular}

Harris Pond 
Upper Reservoir Site:

HSP.B3 24-30

0
24

$\begin{array}{rcc}6 & 3.0 & 1998 \\ 30 & 27.0 & 1968 \\ & & \\ 2.0 & 1.0 & 2000 \\ 4.0 & 3 & 1997.9 \\ 6.0 & 5.0 & 1995.7 \\ 8.0 & 7.0 & 1993.3 \\ 10.0 & 9.0 & 1990.7 \\ 12.0 & 11 & 1987.6 \\ 14.0 & 13.0 & 1984.4 \\ 16.0 & 15.0 & 1980.6 \\ 16.0 & 15.0 & 1980.6 \\ 18.0 & 17.0 & 1976.7 \\ 20.0 & 19.0 & 1973.1 \\ 22.0 & 21.0 & 1969.2 \\ 24.0 & 23.0 & 1965.1 \\ & & \end{array}$

40
50
53
36
30
37
49
41
47
50
47
69
61
68
79
55
104

2

$\begin{array}{ll}49 & 494 \\ 48 & 315\end{array}$

224

1,220

980

422

$720 \quad 710$

Middle Reservoir Site:

HSP.B2 0-2

HSP.B2 2-4

2.0

HSP.B2 6-8

HSP.B2 8-10

HSP.B2 10-12

HSP.B2 12-14

HSP.B2 14-16

HSP.B2 16-18

HSP.B2 18-20

HSP.B2 20-22

Lower Reservoir Site:

HSP. 1 0-6

$24 \quad 21.0$

Mill Creek (Harris Pond inflow):

Mill Creek

$3 / 21$ to $3 / 27 / 01$

122
31

55
40

187

756

665

287

454

462

Mill Creek

$5 / 22$ to $5 / 24 / 01$

585
504
454
512
528
474
458
412
658
416
517
531

566
437
1,660
1,310

100
195
221
238
110
246
241
222
294
245
224
278

262
261

1,370
1,220
1,100
1,220
1,200
1,070
1,000
919
1,380
899
1,040
1,080
1,420
842
4,290
3,890

1,370
1,170
997
906
1,040
1,050
912
880
821
1,230
811
948
991
1,210
832
3,450
2,900

577
518

$969 \quad 946$

$\begin{array}{lll}5182 & 824 & 678 \\ 9 & 406 & 726\end{array}$

$\begin{array}{lll}389 & 655 & 673\end{array}$

$\begin{array}{llll}1,040 & 452 & 737 & 759\end{array}$

$\begin{array}{lllll}1,200 & 1,050 & 488 & 744 & 601\end{array}$

$\begin{array}{llll}912 & 410 & 635 & 654\end{array}$

$\begin{array}{llll}880 & 388 & 621 & 660\end{array}$

$\begin{array}{lllll}1,380 & 1,230 & 519 & 848 & 406\end{array}$

$\begin{array}{lllll}899 & 811 & 342 & 562 & 566\end{array}$

$\begin{array}{lllll}1,040 & 948 & 399 & 641 & 234\end{array}$

$\begin{array}{lllll}1,080 & 991 & 430 & 678 & 697\end{array}$

1,580
2,070

$2,070 \quad 3,190$

2,340 
Calculated Values (Nondetections

treated as zero)

\begin{tabular}{|c|c|c|c|c|c|c|c|}
\hline $\begin{array}{l}\text { Core ID or } \\
\text { Stream }\end{array}$ & Date & $\begin{array}{c}\text { Dibenzo } \\
(\mathrm{a}, \mathrm{h}) \text { anthra } \\
\text { cene }\end{array}$ & $\begin{array}{l}\text { Coro- } \\
\text { nene }\end{array}$ & Total PAH & $\begin{array}{l}\text { Combus- } \\
\text { tion PAH }\end{array}$ & $\begin{array}{c}(2+3) / \\
\text { Combust- } \\
\text { ion PAH }\end{array}$ & Remarks \\
\hline \multicolumn{8}{|c|}{ Como Lake } \\
\hline \multicolumn{8}{|c|}{ Upper Reservoir Site: } \\
\hline CMO.5 & 2000 & 377 & 329 & 30,200 & 22,800 & 0.19 & \\
\hline CMO.5 & 1990 & 345 & 265 & 30,400 & 23,600 & 0.16 & \\
\hline CMO.5 & 1965 & 38 & 10 & 4,320 & 2,980 & 0.33 & \\
\hline \multicolumn{8}{|c|}{ Mid Reservoir Site: } \\
\hline CMO.3 & 1999 & 222 & 68 & 17,600 & 12,800 & 0.27 & \\
\hline CMO.3 & 1970 & 67 & 25 & 12,100 & 4,160 & 1.76 & \\
\hline CMO.3 & 1950 & 8 & 4 & 1,300 & 507 & 1.14 & \\
\hline \multicolumn{8}{|c|}{ Primary (Lower Reservoir) Site: } \\
\hline CMO.1 & 2000.8 & 165 & 79 & 10,800 & 6,850 & 0.42 & \\
\hline CMO.1 & 1999.9 & 299 & 77 & 24,600 & 17,000 & 0.33 & \\
\hline СMO.1 & 1998.7 & 316 & 82 & 20,400 & 12,000 & 0.32 & \\
\hline СMO.1 & 1995.3 & 100 & 72 & 9,080 & 4,920 & 0.64 & \\
\hline CMO.1 & 1990.6 & 132 & 53 & 16,100 & 7,220 & 0.75 & \\
\hline CMO.1 dup & 1990.6 & 82 & 32 & 15,200 & 6,000 & 1.03 & \\
\hline СMO.1 & 1982.5 & 201 & 119 & 17,300 & 10,100 & 0.56 & \\
\hline CMO.1 dup & 1982.5 & 167 & 74 & 16,800 & 9,640 & 0.58 & \\
\hline CMO.1 & 1974.1 & 86 & 20 & 9,280 & 5,260 & 0.63 & \\
\hline СMO.1 & 1969.1 & 100 & 112 & 19,400 & 6,600 & 1.71 & \\
\hline CMO.1 & 1958.6 & 24 & 22 & 5,720 & 1,300 & 2.80 & \\
\hline \multicolumn{8}{|c|}{ Como Lake Inflow: } \\
\hline Lake Como Inflon & 08/30/01 & 1,110 & 1,510 & 94,800 & 63,600 & 0.14 & \\
\hline Lake Como Inflon & 09/20/01 & 983 & 1,240 & 87,500 & 55,000 & 0.20 & \\
\hline Lake Como Inflon & $11 / 09 / 01$ & 779 & 1,640 & 72,700 & 45,900 & 0.22 & \\
\hline Lake Como Inflon & $01 / 23-24 / 02$ & 607 & 510 & 45,800 & 32,100 & 0.16 & \\
\hline
\end{tabular}

Echo Lake 
Upper Reservoir Site:

$\begin{array}{cc}\text { ECO.3 } & 1999 \\ \text { ECO.3 } & 1988 \\ \text { ECO.3 } & 1972 \\ \text { ECO.3 Dup } & 1972\end{array}$

$\begin{array}{ccccc}283 & 231 & 33,200 & 19,500 & 0.33 \\ 180 & 208 & 25,600 & 12,700 & 0.50 \\ 75 & 28 & 19,200 & 6,130 & 1.39 \\ 116 & 30 & 22,600 & 6,480 & 1.63\end{array}$

Middle Reservoir Site:

$\begin{array}{ll}\text { ECO.4 } & 1999 \\ \text { ECO.4 } & 197 \\ \text { ECO.4 } & 1952\end{array}$

ECO 4

ECO.4 dup

1952

$149 \quad 139$

17,700

9,960

$13,600 \quad 3,170 \quad 1,40$

Primary (Lower) Reservoir Site:

$\begin{array}{ll}\text { ECO.1 } & 1999.7 \\ \text { ECO.1 } & 1998.3 \\ \text { ECO.1 } & 1996.4 \\ \text { ECO.1 } & 1991.7 \\ \text { ECO.1 } & 1985.9 \\ \text { ECO.1 } & 1980.6 \\ \text { ECO.1 } & 1972.5 \\ \text { ECO.1 } & 1966.8 \\ \text { ECO.1 } & 1961.1 \\ \text { ECO.1 } & 1951.4\end{array}$

$\begin{array}{lll}4,520 & 1,790 & 0.41\end{array}$

cho Lake Inflow:

Echo Lake Inflow $\quad$ 05/28/01

Echo Lake Inflow $\quad 08 / 17 / 01$

Echo Lake Inflow 09/20/01

$09 / 20 / 01$
$10 / 11 / 01$

$<210$

642

495

Echo Lake Inflow

$10 / 11 / 01 \quad 662$

833
531

17,800

7,090

$8,970 \quad 5,080$

$8,230 \quad 4,330$

$6,740 \quad 3,100$

$13,600 \quad 4,560$

$17,900 \quad 2,530$

$10,400 \quad 2,710$

$5,360 \quad 1,470$

$4,260 \quad 1,320$

$3,560 \quad 1,580$

0.64

0.45

0.50

0.67

1.00
2.96

1.16

1.55

$\begin{array}{lllll}16 & 13 & 4,260 & 1,320 & 0.99\end{array}$

Fosdic Lake

Upper Reservoir Site:

2000

477

$23,500 \quad 4,590$

$54,400 \quad 35,300$

$51,000 \quad 33,000$

0.47

FOS.5 1965

Primary (Mid) Reservoir Site:

$\begin{array}{lcccccc}\text { FOS.4 } & 2000.4 & 217 & 75 & 16,000 & 9,080 & 0.35 \\ \text { FOS.4 } & 1998.4 & 300 & 198 & 22,700 & 13,800 & 0.28 \\ \text { FOS.4 } & 1996.2 & 328 & 439 & 25,900 & 16,500 & 0.24 \\ \text { FOS.4 } & 1991.0 & 138 & 80 & 13,700 & 8,450 & 0.28 \\ \text { FOS.4 } & 1983.3 & 75 & 47 & 9,370 & 4,760 & 0.47 \\ \text { FOS.4 } & 1968.9 & 37 & 30 & 5,330 & 2,030 & 0.82\end{array}$




$\begin{array}{cc}\text { FOS.4.dup } & 1968.9 \\ \text { FOS.4 } & 1959.8 \\ \text { FOS.4 } & 1944.6 \\ \text { FOS.4 } & 1928.3 \\ \text { FOS.4 } & 1912.6\end{array}$

\section{Lower Reservoir Site:}

$\begin{array}{ll}\text { FOS.2 } & 2000 \\ \text { FOS.2 } & 1965 \\ \text { FOS.2 } & 1920\end{array}$

FOS.2 dup

1920

1920

\section{Fosdic Lake Inflow}

Fosdic Lake Inflo। $\quad 08 / 11 / 01$

Fosdic Lake Infloı 09/18/01

Fosdic Lake Inflo। 10/10/01

Fosdic Lake Inflo $\quad 12 / 06 / 01$

30
22
10
5
4

23
16
11
4
4

4,880
4,700
1,470
882
571

$\begin{array}{cc}1,960 & 0.81 \\ 1,390 & 1.28 \\ 498 & 0.97 \\ 226 & 1.57 \\ 186 & 1.14\end{array}$

\section{Upper Mystic Lake}

Upper Lake Site:

UYS Lake Site:

MYS.B2

MYS.B2

MYS.B2

MYS.B2

MYS.B2

MYS.B2

MYS.B2

MYS.B2

MYS.B2

MYS.B2

MYS.B2

MYS.B2

Lower Lake Site:

MYS.2

MYS.2

MYS.2

2000.7
2000.7
2000.7
1999.0
1996.5
1989.1
1982.7
1975.2
1967.9
1958
1958
1943

283

205

142

170
529

Aberjona River (Upper Mystic Lake inflow)

Aberjona River $\quad 3 / 21$ to $3 / 22 / 01 \quad 1,620$

Aberjona River $3 / 22$ to $3 / 23 / 01 \quad 1,060$

Aberjona River $8 / 29$ to $8 / 30 / 02 \quad 1,720$

Harris Pond

105

13

27,200

4,170

15,600

1,420

0.36

7

1,010

1,120

216
242

345

345
103
201

11,800

11,900

17,600

29,600

6,550

7,170

9,990

19,400

0.27

0.30

0.21

\section{8}

1.57
1.14

2.09
2.02

125,000

$\begin{array}{lll}84,900 & 0.19 & 0-6 \mathrm{~cm} \text { mixing zone in core (based on 210Pb) } \\ 47,200 & 0.11 & \text { assigned sampling date assuming }\end{array}$

$56,100 \quad 47,200 \quad 0.11 \quad$ assigned sampling date assuming

$\begin{array}{llll}104,000 & 70,900 & 0.21 & \text { instantaneous mixing. }\end{array}$

$\begin{array}{lll}111,000 & 75,200 & 0.21\end{array}$

$92,200 \quad 61,900 \quad 0.21$

$120,000 \quad 79,800 \quad 0.18$

$108,000 \quad 67,000 \quad 0.24$

$149,000 \quad 66,900 \quad 0.51$

$166,000 \quad 69,200 \quad 0.65$

$154,000 \quad 63,400 \quad 0.62$

$145,000 \quad 60,100 \quad 0.61$

$122,000 \quad 69,500 \quad 0.33$

$\begin{array}{lll}140,000 & 93,600 \quad 0.19\end{array}$

$114,000 \quad 71,900 \quad 0.25$

$140,000 \quad 64,200 \quad 0.71$

$\begin{array}{cccc}455 & 135,000 & 91,300 & 0.22 \\ 1,600 & 147,000 & 98,000 & 0.22\end{array}$

$\begin{array}{llll}1,570 & 175,000 & 102,000 & 0.22\end{array}$

Mean of these two samples over 3-day event used to represent event. 
Upper Reservoir Site:

\begin{tabular}{|c|c|c|c|c|c|c|}
\hline \\
\hline HSP.B3 0-6 & 1998 & 159 & 55 & 14,900 & 6,750 & 0.50 \\
\hline HSP.B3 24-30 & 1968 & 100 & 40 & 10,700 & 4,270 & 0.66 \\
\hline \multicolumn{7}{|c|}{ Middle Reservoir Site: } \\
\hline HSP.B2 0-2 & 2000 & 179 & 62 & 22,100 & 9,160 & 0.49 \\
\hline HSP.B2 2-4 & 1997.9 & 68 & 7 & 12,500 & 7,230 & 0.37 \\
\hline HSP.B2 4-6 & 1995.7 & 148 & 67 & 14,200 & 6,870 & 0.44 \\
\hline HSP.B2 6-8 & 1993.3 & 138 & 59 & 13,900 & 6,070 & 0.53 \\
\hline HSP.B2 8-10 & 1990.7 & 160 & 71 & 15,800 & 6,960 & 0.54 \\
\hline HSP.B2 10-12 & 1987.6 & 76 & 9 & 11,700 & 6,460 & 0.40 \\
\hline HSP.B2 12-14 & 1984.4 & 128 & 39 & 14,100 & 6,000 & 0.57 \\
\hline HSP.B2 14-16 & 1980.6 & 111 & 32 & 12,700 & 5,850 & 0.58 \\
\hline HSP.B2 14-16 du & 1980.6 & 102 & 31 & 12,200 & 5,390 & 0.55 \\
\hline HSP.B2 16-18 & 1976.7 & 132 & 45 & 17,300 & 7,740 & 0.57 \\
\hline HSP.B2 18-20 & 1973.1 & 130 & 41 & 13,500 & 5,330 & 0.69 \\
\hline HSP.B2 20-22 & 1969.2 & 136 & 36 & 15,200 & 5,590 & 0.58 \\
\hline HSP.B2 22-24 & 1965.1 & 85 & 42 & 15,800 & 6,490 & 0.67 \\
\hline \multicolumn{7}{|c|}{ Lower Reservoir Site: } \\
\hline HSP.1 0-6 & 1997 & 172 & 52 & 17,200 & 7,920 & 0.50 \\
\hline HSP.1 18-24 & 1966 & 134 & 46 & 15,500 & 5,450 & 0.85 \\
\hline \multicolumn{7}{|c|}{ Mill Creek (Harris Pond inflow): } \\
\hline Mill Creek & $3 / 21$ to $3 / 27 / 01$ & 368 & 280 & 44,000 & 23,600 & 0.42 \\
\hline Mill Creek & $5 / 22$ to $5 / 24 / 01$ & 467 & 441 & 40,000 & 27,400 & 0.13 \\
\hline
\end{tabular}


Table B1. Organochlorine compound quality control samples.

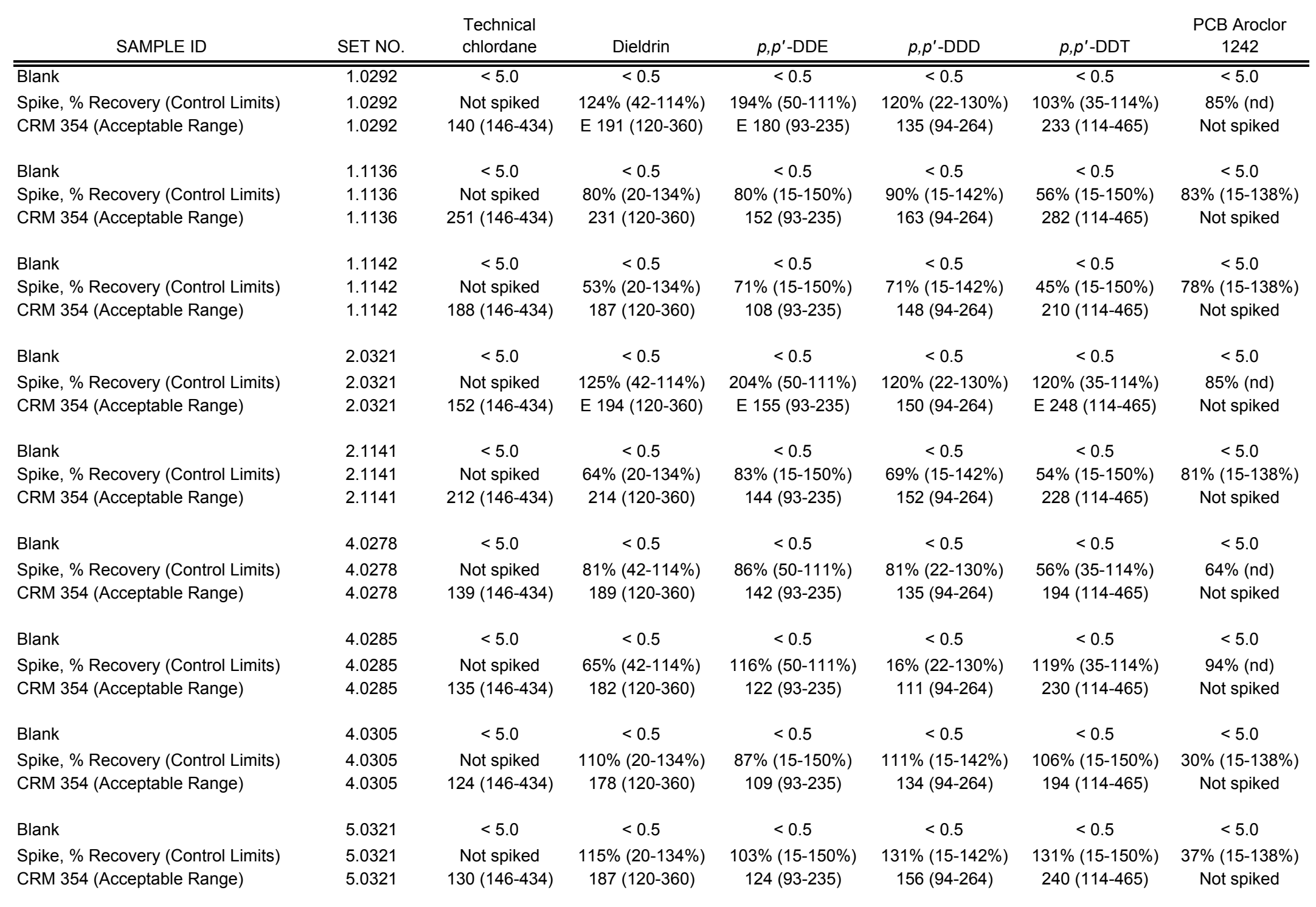




\begin{tabular}{|c|c|c|c|c|c|c|c|}
\hline SAMPLE ID & SET NO. & $\begin{array}{l}\text { Technical } \\
\text { chlordane }\end{array}$ & Dieldrin & $p, p^{\prime}-\mathrm{DDE}$ & $p, p^{\prime}$-DDD & $p, p^{\prime}$-DDT & $\begin{array}{c}\text { PCB Aroclor } \\
1242\end{array}$ \\
\hline Blank & 5.1137 & $<5.0$ & $<0.5$ & $<0.5$ & $<0.5$ & $<0.5$ & $<5.0$ \\
\hline Spike, \% Recovery (Control Limits) & 5.1137 & Not spiked & $77 \%(20-134 \%)$ & $91 \%(15-150 \%)$ & $89 \%(15-142 \%)$ & $61 \%(15-150 \%)$ & $83 \%(15-138 \%)$ \\
\hline CRM 354 (Acceptable Range) & 5.1137 & $225(146-434)$ & $216(120-360)$ & $158(93-235)$ & $162(94-264)$ & $241(114-465)$ & Not spiked \\
\hline Blank & 6.0319 & $<5.0$ & $<0.5$ & $<0.5$ & $<0.5$ & $<0.5$ & $<5.0$ \\
\hline Spike, \% Recovery (Control Limits) & 6.0319 & Not spiked & $116 \%(20-134 \%)$ & $179 \%(15-150 \%)$ & $121 \%(15-142 \%)$ & $131 \%(15-150 \%)$ & $93 \%(15-138 \%)$ \\
\hline CRM 354 (Acceptable Range) & 6.0319 & $151(146-434)$ & $196(120-360)$ & E $158(93-235)$ & $162(94-264)$ & $256(114-465)$ & Not spiked \\
\hline Blank & 7.1120 & $<5.0$ & $<0.5$ & $<0.5$ & $<0.5$ & $<0.5$ & $<5.0$ \\
\hline Spike, \% Recovery (Control Limits) & 7.1120 & Not spiked & $72 \%(20-134 \%)$ & $100 \%(15-150 \%)$ & $80 \%(15-142 \%)$ & $63 \%(15-150 \%)$ & $89 \%(15-138 \%)$ \\
\hline CRM 354 (Acceptable Range) & 7.1120 & $178(146-434)$ & $212(120-360)$ & $139(93-235)$ & $162(94-264)$ & $279(114-465)$ & Not spiked \\
\hline Blank & 200120107 & $<5.0$ & $<0.5$ & $<0.5$ & $<0.5$ & $<0.5$ & $<5.0$ \\
\hline Spike, \% Recovery (Control Limits) & 200120107 & Not spiked & $72 \%(20-134 \%)$ & $90 \%(15-150 \%)$ & $88 \%(15-142 \%)$ & $59 \%(15-150 \%)$ & $80 \%(15-138 \%)$ \\
\hline CRM 354 (Acceptable Range) & 200120107 & Not spiked & $242(120-360)$ & $152(93-235)$ & $174(94-264)$ & $262(114-465)$ & Not spiked \\
\hline CRM 354 (Acceptable Range) & 200120409 & Not spiked & $195(120-360)$ & $130(93-235)$ & $148(94-264)$ & $249(114-465)$ & Not spiked \\
\hline Blank & 200121505 & $<5.0$ & $<0.5$ & $<0.5$ & $<0.5$ & $<0.5$ & $<5.0$ \\
\hline Spike, \% Recovery (Control Limits) & 200121505 & Not spiked & $66 \%(20-134 \%)$ & $113 \%(15-150 \%)$ & $83 \%(15-142 \%)$ & $76 \%(15-150 \%)$ & $106 \%(15-138 \%)$ \\
\hline CRM 354 (Acceptable Range) & 200121505 & Not spiked & $185(120-360)$ & $166(93-235)$ & $150(94-264)$ & $223(114-465)$ & Not spiked \\
\hline Blank & 200122506 & $<5.0$ & $<0.5$ & $<0.5$ & $<0.5$ & $<0.5$ & $<5.0$ \\
\hline Spike, \% Recovery (Control Limits) & 200122506 & Not spiked & $56 \%(20-134 \%)$ & $83 \%(15-150 \%)$ & $67 \%(15-142 \%)$ & $62 \%(15-150 \%)$ & $73 \%(15-138 \%)$ \\
\hline CRM 354 (Acceptable Range) & 200122506 & Not spiked & $190(120-360)$ & $163(93-235)$ & $173(94-264)$ & $283(114-465)$ & Not spiked \\
\hline Blank & 200128405 & $<5.0$ & $<0.5$ & $<0.5$ & $<0.5$ & $<0.5$ & $<5.0$ \\
\hline Spike, \% Recovery (Control Limits) & 200128405 & Not spiked & $67 \%(20-134 \%)$ & $126 \%(15-150 \%)$ & $66 \%(15-142 \%)$ & $65 \%(15-150 \%)$ & $112 \%(15-138 \%)$ \\
\hline CRM 354 (Acceptable Range) & 200128405 & Ruined in prepar & tion & & & & \\
\hline Blank & 200205006 & $<5.0$ & $<0.5$ & $<0.5$ & $<0.5$ & $<0.5$ & $<5.0$ \\
\hline Spike, \% Recovery (Control Limits) & 200205006 & Not spiked & $76 \%(15-150 \%)$ & $77 \%(34-140 \%)$ & $72 \%(22-158 \%)$ & $63 \%(15-150 \%)$ & $73 \%(17-145 \%)$ \\
\hline CRM 362 (Acceptable Range) & 200205006 & Not spiked & $142(93-256)$ & $101(74-194)$ & $199(120-401)$ & $128(94-261)$ & Not spiked \\
\hline Spike, \% Recovery (Control Limits) & 200207106 & Not spiked & $94 \%(15-150 \%)$ & $89 \%(34-140 \%)$ & $66 \%(22-158 \%)$ & $72 \%(15-150 \%)$ & $77 \%(17-145 \%)$ \\
\hline
\end{tabular}




\begin{tabular}{|c|c|c|c|c|c|c|c|}
\hline SAMPLE ID & SET NO. & $\begin{array}{l}\text { Technical } \\
\text { chlordane }\end{array}$ & Dieldrin & $p, p^{\prime}-\mathrm{DDE}$ & $p, p^{\prime}-\mathrm{DDD}$ & $p, p^{\prime}$-DDT & $\begin{array}{c}\text { PCB Aroclor } \\
1242\end{array}$ \\
\hline CRM 362 (Acceptable Range) & 200207106 & Not spiked & $165(93-256)$ & $115(74-194)$ & $182(120-401)$ & $155(94-261)$ & Not spiked \\
\hline Blank & 200207706 & $<5.0$ & $<0.5$ & $<0.5$ & $<0.5$ & $<0.5$ & $<5.0$ \\
\hline Spike, \% Recovery (Control Limits) & 200207706 & Not spiked & $68 \%(15-150 \%)$ & $97 \%(34-140 \%)$ & $61 \%(22-158 \%)$ & $65 \%(15-150 \%)$ & $88 \%(17-145 \%)$ \\
\hline CRM 362 (Acceptable Range) & 200207706 & Not spiked & $129(93-256)$ & $104(74-194)$ & $161(120-401)$ & $131(94-261)$ & Not spiked \\
\hline Blank & 200208407 & $<5.0$ & $<0.5$ & $<0.5$ & $<0.5$ & $<0.5$ & $<5.0$ \\
\hline Spike, \% Recovery (Control Limits) & 200208407 & Not spiked & $76 \%(15-150 \%)$ & $94 \%(34-140 \%)$ & $52 \%(22-158 \%)$ & $69 \%(15-150 \%)$ & $78 \%(17-145 \%)$ \\
\hline CRM 362 (Acceptable Range) & 200208407 & Not spiked & $144(93-256)$ & $109(74-194)$ & $141(120-401)$ & $138(94-261)$ & Not spiked \\
\hline
\end{tabular}


SPIKE

RECOVERY

SAMPLE ID SET NO. PCB Aroclor 1254 PCB Aroclor 1260 (\%) FOR SET

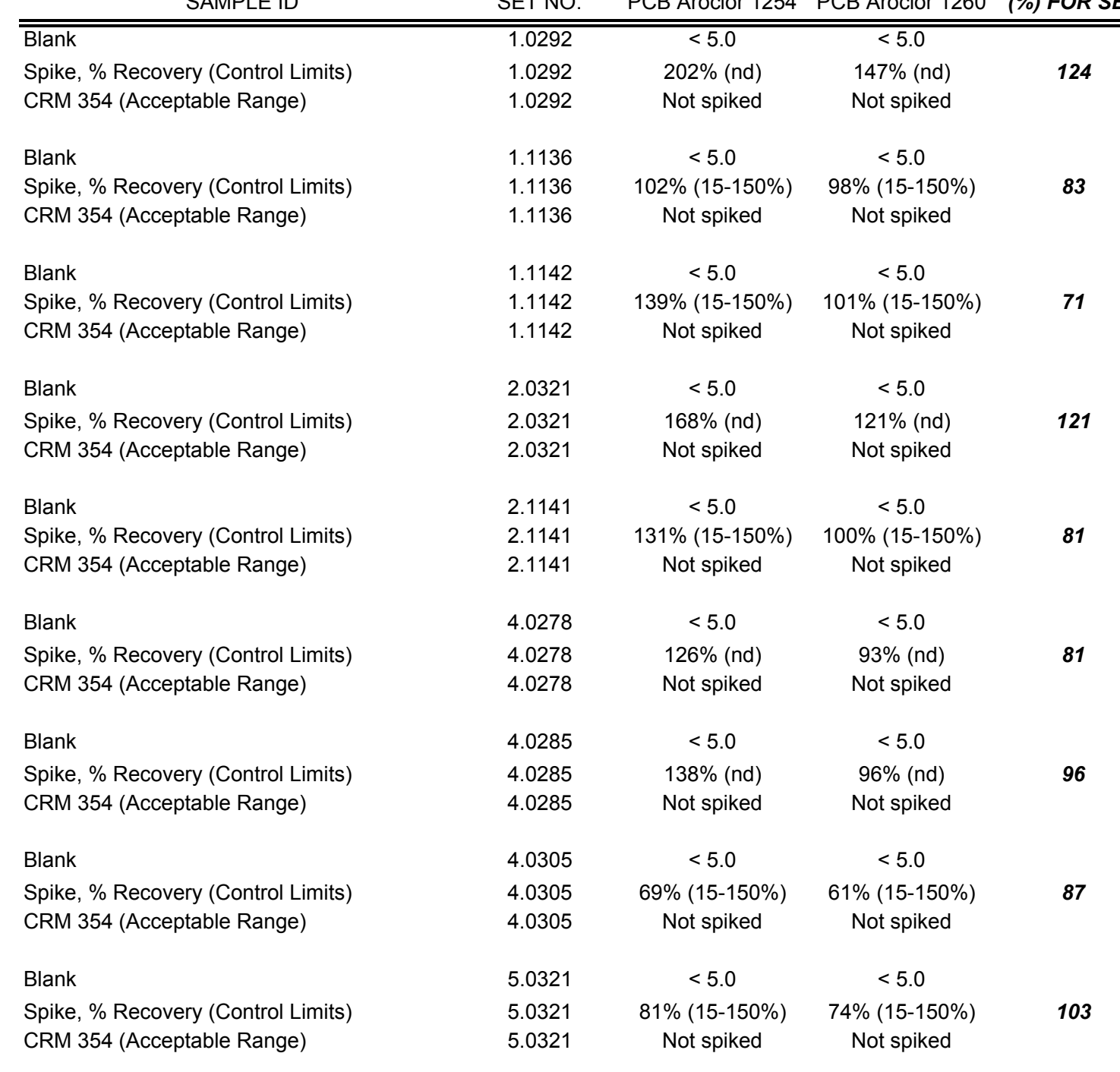


MEDIAN SPIKE

RECOVERY

\begin{tabular}{|c|c|c|c|c|}
\hline SAMPLE ID & SET NO. & PCB Aroclor 1254 & PCB Aroclor 1260 & $\begin{array}{l}\text { RECOVEF } \\
\text { (\%) FOR S }\end{array}$ \\
\hline Blank & 5.1137 & $<5.0$ & $<<5.0$ & \\
\hline Spike, \% Recovery (Control Limits) & 5.1137 & $118 \%(15-150 \%)$ & $98 \%(15-150 \%)$ & 89 \\
\hline CRM 354 (Acceptable Range) & 5.1137 & Not spiked & Not spiked & \\
\hline Blank & 6.0319 & $<5.0$ & $<5.0$ & \\
\hline Spike, \% Recovery (Control Limits) & 6.0319 & $185 \%(15-150 \%)$ & $135 \%(15-150 \%)$ & 131 \\
\hline CRM 354 (Acceptable Range) & 6.0319 & Not spiked & Not spiked & \\
\hline Blank & 7.1120 & $<5.0$ & $<5.0$ & \\
\hline Spike, \% Recovery (Control Limits) & 7.1120 & $133 \%(15-150 \%)$ & $123 \%(15-150 \%)$ & 89 \\
\hline CRM 354 (Acceptable Range) & 7.1120 & Not spiked & Not spiked & \\
\hline Blank & 200120107 & $<5.0$ & $<5.0$ & \\
\hline Spike, \% Recovery (Control Limits) & 200120107 & $145 \%(15-150 \%)$ & $102 \%(15-150 \%)$ & 88 \\
\hline CRM 354 (Acceptable Range) & 200120107 & Not spiked & Not spiked & \\
\hline Blank & 200120409 & $<5.0$ & $<5.0$ & \\
\hline Spike, \% Recovery (Control Limits) & 200120409 & $143 \%(15-150 \%)$ & $100 \%(15-150 \%)$ & 83 \\
\hline CRM 354 (Acceptable Range) & 200120409 & Not spiked & Not spiked & \\
\hline Blank & 200121505 & $<5.0$ & $<5.0$ & \\
\hline Spike, \% Recovery (Control Limits) & 200121505 & $138 \%(15-150 \%)$ & $98 \%(15-150 \%)$ & 98 \\
\hline CRM 354 (Acceptable Range) & 200121505 & Not spiked & Not spiked & \\
\hline Blank & 200122506 & $<5.0$ & $<5.0$ & \\
\hline Spike, \% Recovery (Control Limits) & 200122506 & $136 \%(15-150 \%)$ & $96 \%(15-150 \%)$ & 73 \\
\hline CRM 354 (Acceptable Range) & 200122506 & Not spiked & Not spiked & \\
\hline Blank & 200128405 & $<5.0$ & $<5.0$ & \\
\hline Spike, \% Recovery (Control Limits) & 200128405 & $244 \%(15-150 \%)$ & $145 \%(15-150 \%)$ & 112 \\
\hline CRM 354 (Acceptable Range) & 200128405 & & & \\
\hline Blank & 200205006 & $<5.0$ & $<5.0$ & \\
\hline Spike, \% Recovery (Control Limits) & 200205006 & $87 \%(53-150 \%)$ & $80 \%(33-150 \%)$ & 76 \\
\hline CRM 362 (Acceptable Range) & 200205006 & Not spiked & Not spiked & \\
\hline Blank & 200207106 & $<5.0$ & $<5.0$ & \\
\hline Spike, \% Recovery (Control Limits) & 200207106 & $88 \%(53-150 \%)$ & $90 \%(33-150 \%)$ & 88 \\
\hline
\end{tabular}


MEDIAN SPIKE

RECOVERY

SAMPLE ID

SET NO.

PCB Aroclor $1254 \quad$ PCB Aroclor $1260 \quad$ (\%) FOR SET

CRM 362 (Acceptable Range)

200207106

Not spiked

Not spiked

\section{Blank}

Spike, \% Recovery (Control Limits)

CRM 362 (Acceptable Range)

Blank

Spike, \% Recovery (Control Limits)

CRM 362 (Acceptable Range)

200207706

$<5.0$

$<5.0$

200207706

$84 \%(53-15) \%$

$94 \%(33-150 \%)$

200207706

Not spiked

Not spiked

200208407

200208407

200208407

$<5.0$

$<5.0$

$84 \%(53-150 \%) \quad 86 \%(33-150 \%)$

Not spiked

Not spiked 
Table B2. Chlorinated hydrocarbon duplicate samples.

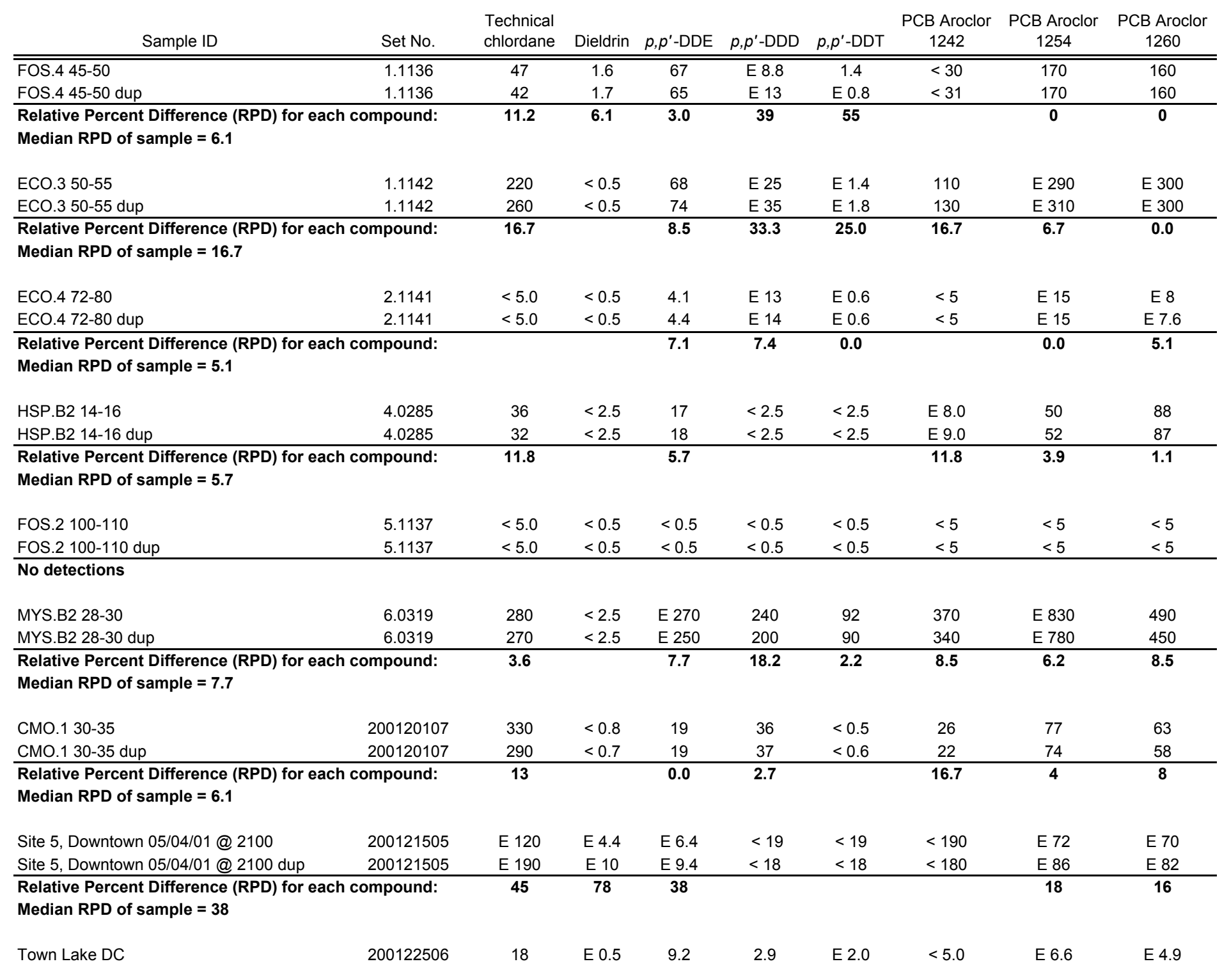




\begin{tabular}{|c|c|c|c|c|c|c|c|c|c|}
\hline Sample ID & Set No. & $\begin{array}{l}\text { Technical } \\
\text { chlordane }\end{array}$ & Dieldrin & $p, p^{\prime}-\mathrm{DDE}$ & $p, p^{\prime}-\mathrm{DDD}$ & $p, p^{\prime}-\mathrm{DDT}$ & $\begin{array}{l}\text { PCB Aroclor } \\
1242\end{array}$ & $\begin{array}{l}\text { PCB Aroclor } \\
1254\end{array}$ & $\begin{array}{l}\text { PCB Aroclor } \\
1260\end{array}$ \\
\hline Town Lake DC dup & 200122506 & 17 & E 0.5 & 9.4 & 2.5 & E 1.4 & $<5.0$ & E 5.6 & E 4.8 \\
\hline \multicolumn{10}{|c|}{ Median RPD of sample $=5.7$} \\
\hline CMO.1 45-50 & 200128405 & 185 & 1.8 & E 17 & $<0.5$ & 0.85 & E 33 & 57 & 26 \\
\hline CMO.1 45-50 dup & 200128405 & 180 & 2.2 & E 16 & $<0.5$ & 1.5 & E 34 & 54 & 23 \\
\hline $\begin{array}{l}\text { Relative Percent Difference (RPD) for } \epsilon \\
\text { Median RPD of sample }=6.1\end{array}$ & ompound: & 2.7 & 20 & 6.1 & & 55 & 3.0 & 5.4 & 12 \\
\hline Site 5, Downtown 08/16/01 @ 0846 & 200205006 & 150 & E 2.0 & E 11 & $<10$ & E 9.3 & E 82 & 310 & 350 \\
\hline Site 5, Downtown 08/16/01@ 0847 dup & 200205006 & 160 & 5.8 & E 9.4 & $<4.0$ & 13 & 64 & 470 & 230 \\
\hline \multicolumn{9}{|l|}{ Median RPD of sample $=\mathbf{3 3}$} & 41 \\
\hline LKH.5 0-5 & 200207706 & $<20$ & $<2.0$ & 4.9 & E 3.3 & E 5.4 & E 15 & $<20$ & $<20$ \\
\hline LKH.5 0-5 dup & 200207706 & $<20$ & $<2.0$ & 4.8 & E 3.4 & E 25 & E 14 & $<20$ & $<20$ \\
\hline \multicolumn{10}{|c|}{ Median RPD of sample $=4.9$} \\
\hline Onion Crk. 11/15/01 @ 1500 & 200208407 & $<10$ & $<1.0$ & E 0.43 & $<1.0$ & $<1.0$ & $<10$ & $<10$ & $<10$ \\
\hline Onion Crk.11/15/01 @ 1500 dup & 200208407 & $<10$ & $<1.0$ & E 0.45 & $<1.0$ & $<1.0$ & $<10$ & $<10$ & $<10$ \\
\hline \multicolumn{10}{|l|}{ Median RPD of sample $=4.5$} \\
\hline Barton Above & 200222605 & $<65$ & $<6.5$ & E 0.83 & $<6.5$ & $<6.5$ & $<65$ & $<65$ & $<65$ \\
\hline Barton Above dup & 200222605 & $<90$ & $<9.0$ & E 1.0 & $<9.0$ & $<9.0$ & $<90$ & $<90$ & $<90$ \\
\hline $\begin{array}{l}\text { Relative Percent Difference (RPD) for } \epsilon \\
\text { Median RPD of sample }=19\end{array}$ & ompound: & & & 18.6 & & & & & \\
\hline MIL.B3 10-15 ref1 & 200226710 & $<20$ & $<2.0$ & E 0.62 & E 1.3 & $<2.0$ & $<20$ & $<20$ & $<20$ \\
\hline MIL.B3 10-15 ref1lab dup & 200226710 & $<20$ & $<2.0$ & E 0.47 & E 0.92 & $<2.0$ & $<20$ & $<20$ & $<20$ \\
\hline $\begin{array}{l}\text { Relative Percent Difference (RPD) for } \epsilon \\
\text { Median RPD of sample }=30.9\end{array}$ & ompound: & & & 27.5 & 34.2 & & & & \\
\hline $\begin{array}{l}\text { MEDIAN RPD FOR EAC } \\
\text { MEDIAN RPD FOR ALL } \\
\text { DUPLICATES IN STUDY }=8.5\end{array}$ & MPOUND: & 11.2 & 20.0 & 6.6 & 16.5 & 34.2 & 11.8 & 5.8 & 6.7 \\
\hline
\end{tabular}




\section{Table B3. Polycyclic aromatic hydrocarbon quality control samples.}

\begin{tabular}{|c|c|c|c|c|c|c|c|c|}
\hline Sample ID & Batch & Naphthalene & $\begin{array}{c}9 \mathrm{H}- \\
\text { Fluorene }\end{array}$ & Phenanthrene & Anthracene & Fluoranthene & Pyrene & $\begin{array}{c}\text { Benz (a) } \\
\text { Anthracene }\end{array}$ \\
\hline Blank & $\mathrm{P} 8022 \mathrm{H} 00292$ & E0.11 & $<<5$ & E0.11 & $<<5$ & E0.040 & $\overline{E 0.021}$ & $<<5$ \\
\hline Spike, \% Recovery & $8022 \mathrm{H} 00292$ & 80.06 & 81.68 & 86.55 & 80.25 & 92.51 & 91.33 & 95.32 \\
\hline CRM 354, \% Recovery (Acceptable Range) & $8022 \mathrm{H} 00292$ & $41.72(15-95)$ & & $69.16(39-94)$ & $46.80(18-95)$ & $68.95(33-90)$ & $70.40(32-90)$ & \\
\hline Blank & $8022 R 01136$ & $<5$ & $<5$ & $<5$ & $<5$ & $<5$ & $<5$ & $<5$ \\
\hline Spike, \% Recovery & $8022 R 01136$ & 57.78 & 65.85 & 70.78 & 62.03 & 84.84 & 84.24 & 91.76 \\
\hline CRM 354, \% Recovery (Acceptable Range) & $8022 R 01136$ & $46.15(15-95)$ & & $82.44(39-94)$ & $50.40(18-95)$ & $87.77(33-90)$ & $84.57(32-90)$ & \\
\hline Blank & 8022R01142 & $<5$ & $<5$ & $<5$ & $<5$ & $<5$ & $<5$ & $<5$ \\
\hline Spike, \% Recovery & $8022 R 01142$ & 34.57 & 45.20 & 64.77 & 55.22 & 75.08 & 75.20 & 72.87 \\
\hline CRM 354, \% Recovery (Acceptable Range) & 8022R01142 & $34.45(15-95)$ & & $64.48(39-94)$ & $40.19(18-95)$ & $79.57(33-90)$ & $78.14(32-90)$ & \\
\hline Blank & $8022 \mathrm{H} 00322$ & E0.15 & $<5$ & $<5$ & $<5$ & $<5$ & $<5$ & $<5$ \\
\hline Spike, \% Recovery & $8022 \mathrm{H} 00322$ & 79.97 & 79.51 & 83.29 & 62.08 & 92.37 & 93.59 & 94.18 \\
\hline CRM 354, \% Recovery (Acceptable Range) & $8022 \mathrm{H} 00322$ & $63.67(15-95)$ & & $81.37(39-94)$ & $47.58(18-95)$ & $78.83(33-90)$ & $82.14(32-90)$ & \\
\hline Blank & $8022 R 01141$ & E0.28 & $<5$ & $<5$ & $<5$ & $<5$ & $<5$ & $<5$ \\
\hline Spike, \% Recovery & $8022 R 01141$ & 69.68 & 61.95 & 73.08 & 60.74 & 73.97 & 73.34 & 72.77 \\
\hline CRM 354, \% Recovery (Acceptable Range) & $8022 \mathrm{R} 01141$ & $43.33(15-95)$ & & $73.72(39-94)$ & $43.23(18-95)$ & $74.30(33-90)$ & $72.14(32-90)$ & \\
\hline Blank & 8022R00299 & E0.093 & $<5$ & $<5$ & $<5$ & $<5$ & $<5$ & $<5$ \\
\hline Spike, \% Recovery & 8022R00299 & 86.89 & 87.25 & 89.73 & 77.81 & 96.23 & 95.3 & 93.16 \\
\hline CRM 354, \% Recovery (Acceptable Range) & 8022R00299 & $34.98(15-95)$ & & $76.15(39-94)$ & $52.34(18-95)$ & $73.27(33-90)$ & $77.71(32-90)$ & \\
\hline Blank & $8022 R 00285$ & E0.35 & $<5$ & $<5$ & $<5$ & $<5$ & $<5$ & $<5$ \\
\hline Spike, \% Recovery & $8022 R 00285$ & 85.02 & 85.35 & 89.03 & 80.66 & 99.75 & 98.09 & 104.44 \\
\hline CRM 354, \% Recovery (Acceptable Range) & $8022 \mathrm{R} 00285$ & $52.20(15-95)$ & & $86.21(39-94)$ & $57.49(18-95)$ & $94.87(33-90)$ & $92.35(32-90)$ & \\
\hline Blank & $8022 \mathrm{H} 00305$ & $<5$ & $<5$ & E0.071 & $<5$ & $<5$ & $<5$ & $<5$ \\
\hline Spike, \% Recovery & $8022 \mathrm{H} 00305$ & 72.30 & 74.35 & 78.84 & 65.33 & 85.08 & 85.27 & 92.67 \\
\hline CRM 354, \% Recovery (Acceptable Range) & $8022 \mathrm{H} 00305$ & $44.57(15-95)$ & & $83.26(39-94)$ & $57.29(18-95)$ & $79.49(33-90)$ & $81.64(32-90)$ & \\
\hline Blank & $8022 \mathrm{H} 00321$ & E0.10 & $<5$ & $<5$ & $<5$ & $<5$ & $<5$ & $<5$ \\
\hline Spike, \% Recovery & $8022 \mathrm{H} 00321$ & 54.49 & 77.80 & 83.77 & 70.94 & 95.77 & 95.74 & 104.72 \\
\hline CRM 354, \% Recovery (Acceptable Range) & $8022 \mathrm{H} 00321$ & $44.85(15-95)$ & & $66.91(39-94)$ & $44.93(18-95)$ & $67.42(33-90)$ & $69.55(32-90)$ & \\
\hline
\end{tabular}




\begin{tabular}{|c|c|c|c|c|c|c|c|c|}
\hline Sample ID & Batch & Naphthalene & $\begin{array}{c}9 \mathrm{H}- \\
\text { Fluorene }\end{array}$ & Phenanthrene & Anthracene & Fluoranthene & Pyrene & $\begin{array}{c}\text { Benz (a) } \\
\text { Anthracene }\end{array}$ \\
\hline Blank & 8022R01137 & $<<5$ & $<<5$ & $<<5$ & $<<5$ & $2<5$ & $<<5$ & $<<5$ \\
\hline Spike, \% Recovery & $8022 \mathrm{R} 01137$ & 62.17 & 69.54 & 71.17 & 54.21 & 81.42 & 80.50 & 65.68 \\
\hline CRM 354, \% Recovery (Acceptable Range) & $8022 R 01137$ & $54.53(15-95)$ & & $75.76(39-94)$ & $46.21(18-95)$ & $85.52(33-90)$ & $83.98(32-90)$ & \\
\hline Blank & $8022 R 01002$ & E0.29 & $<5$ & $<5$ & $<5$ & $<5$ & $<5$ & $<5$ \\
\hline Spike, \% Recovery & 8022R01002 & 87.14 & 81.17 & 93.35 & 83.71 & 93.16 & 92.04 & 93 \\
\hline CRM 354, \% Recovery (Acceptable Range) & $8022 R 01002$ & $78.77(15-95)$ & & $95.78(39-94)$ & $62.14(18-95)$ & $96.29(33-90)$ & $92.10(32-90)$ & \\
\hline Blank & $8022 R 01112$ & E0.29 & $<5$ & $<5$ & $<5$ & $<5$ & $<5$ & $<5$ \\
\hline Spike, \% Recovery & $8022 R 01112$ & 62.77 & 57.51 & 65.05 & 60.11 & 71.72 & 73.41 & 64.32 \\
\hline CRM 354, \% Recovery (Acceptable Range) & $8022 R 01112$ & $55.14(15-95)$ & & $77.74(39-94)$ & $50.18(18-95)$ & $87.23(33-90)$ & $84.57(32-90)$ & \\
\hline Blank & $8022 R 01206$ & $<5$ & $<5$ & $<5$ & $<5$ & $<5$ & $<5$ & $<5$ \\
\hline Spike, \% Recovery & $8022 R 01206$ & 57.44 & 62.25 & 66.70 & 53.67 & 66.29 & 65.60 & 73.57 \\
\hline CRM 354, \% Recovery (Acceptable Range) & $8022 R 01206$ & $38.75(15-95)$ & & $60.11(39-94)$ & $37.40(18-95)$ & $66.83(33-90)$ & $64.73(32-90)$ & \\
\hline Blank & $8022 R 01205$ & E0.17 & $<5$ & $<5$ & $<5$ & $<5$ & $<5$ & $<5$ \\
\hline Spike, \% Recovery & $8022 R 01205$ & 51.65 & 62.72 & 64.42 & 50.02 & 67.27 & 67.92 & 87.29 \\
\hline CRM 354, \% Recovery (Acceptable Range) & $8022 R 01205$ & $35.37(15-95)$ & & $75.59(39-94)$ & $42.99(18-95)$ & $76.59(33-90)$ & $72.48(32-90)$ & \\
\hline Blank & $8022 \mathrm{R} 01215$ & E0.020 & $<5$ & E0.16 & $<5$ & $<5$ & $<5$ & $<5$ \\
\hline Spike, \% Recovery & $8022 R 01215$ & 60.22 & 60.00 & 63.88 & 53.26 & 68.50 & 68.91 & 72.53 \\
\hline CRM 354, \% Recovery (Acceptable Range) & $8022 R 01215$ & $37.18(15-95)$ & & $62.45(39-94)$ & $37.24(18-95)$ & $66.69(33-90)$ & $65.93(32-90)$ & \\
\hline Blank & $8022 R 01164$ & E0.085 & $<5$ & $<5$ & $<5$ & $<5$ & $<5$ & $<5$ \\
\hline Spike, \% Recovery & $8022 R 01164$ & 52.95 & 63.50 & 67.84 & 41.83 & 68.06 & 65.90 & 64.97 \\
\hline CRM 354, \% Recovery (Acceptable Range) & $8022 R 01164$ & $40.99(15-95)$ & & $67.83(39-94)$ & $35.65(18-95)$ & $78.23(33-90)$ & $75.13(32-90)$ & \\
\hline Blank & $8022 R 01234$ & $<5$ & $<5$ & $<5$ & $<5$ & $<5$ & $<5$ & $<5$ \\
\hline Spike, \% Recovery & $8022 R 01234$ & 67.29 & 67.01 & 72.40 & 50.62 & E69.34 & E67.77 & 65.44 \\
\hline CRM 354, \% Recovery (Acceptable Range) & $8022 R 01234$ & & Ruined in $p$ & eparation & & & & Ruined in prep \\
\hline Blank & $8022 R 01235$ & $<5$ & $<5$ & $<5$ & $<5$ & $<5$ & $<5$ & $<5$ \\
\hline Spike, \% Recovery & $8022 R 01235$ & 67.20 & 79.37 & 75.51 & 67.44 & 78.30 & 76.56 & 96.79 \\
\hline CRM 362, \% Recovery (Acceptable Range) & $8022 R 01235$ & $52.92(0-117)$ & & $65.92(44-124)$ & $52.64(38-105)$ & $70.99(43-125)$ & $68.25(32-139)$ & \\
\hline Blank & $8022 R 01261$ & $<5$ & $<5$ & $<5$ & $<5$ & $<5$ & $<5$ & $<5$ \\
\hline Spike, \% Recovery & $8022 \mathrm{R} 01261$ & 55.23 & 64.91 & 71.44 & 67.40 & 80.86 & 81.86 & 80.39 \\
\hline
\end{tabular}




\begin{tabular}{|c|c|c|c|c|c|c|c|c|}
\hline Sample ID & Batch & Naphthalene & $\begin{array}{c}9 \mathrm{H}- \\
\text { Fluorene }\end{array}$ & Phenanthrene & Anthracene & Fluoranthene & Pyrene & $\begin{array}{c}\text { Benz (a) } \\
\text { Anthracene }\end{array}$ \\
\hline CRM 362, \% Recovery (Acceptable Range) & $8022 \mathrm{R} 01261$ & $54.65(0-117)$ & & $74.50(44-124)$ & $60.30(38-105)$ & $82.70(43-125)$ & $81.29(32-139)$ & \\
\hline Blank & 8022R01243 & $<5$ & $<5$ & $<5$ & $<5$ & $<5$ & $<5$ & $<5$ \\
\hline Spike, \% Recovery & $8022 R 01243$ & 67.69 & 62.31 & 68.05 & 60.28 & 75.75 & 76.80 & 74.30 \\
\hline CRM 362, \% Recovery (Acceptable Range) & $8022 \mathrm{R} 01243$ & $59.18(0-117)$ & & $69.14(44-124)$ & $59.24(38-105)$ & $76.88(43-125)$ & $75.44(32-139)$ & \\
\hline Blank & 8022R02192 & $<5$ & $<5$ & $<5$ & $<5$ & $<5$ & $<5$ & $<5$ \\
\hline Spike, \% Recovery & 8022R02192 & 61.82 & 68.26 & 72.47 & 62.92 & 72.81 & 72.41 & 74.85 \\
\hline CRM 362, \% Recovery (Acceptable Range) & 8022R02192 & $43.38(0-117)$ & & $63.45(44-124)$ & $50.36(38-105)$ & $66.23(43-125)$ & $65.12(32-139)$ & \\
\hline
\end{tabular}


MEDIAN SPIKE

RECOVERY (\%)

Sample ID

Batch

Chrysene Benzo (a) pyrene Coronene

FOR BATCH

\begin{tabular}{|c|c|c|c|c|c|}
\hline Blank & $8022 \mathrm{H} 00292$ & $<5$ & $<5$ & $<5$ & \\
\hline Spike, \% Recovery & $8022 \mathrm{H} 00292$ & 96.38 & 84.17 & E7.31 & 85.36 \\
\hline CRM 354, \% Recovery (Acceptable Range) & $8022 \mathrm{H} 00292$ & $78.77(41-90)$ & & & \\
\hline Blank & 8022R01136 & $<5$ & $<5$ & $<5$ & \\
\hline Spike, \% Recovery & $8022 \mathrm{R} 01136$ & 80.22 & 74.09 & E43.00 & 72.44 \\
\hline CRM 354, \% Recovery (Acceptable Range) & $8022 \mathrm{R} 01136$ & $78.98(41-90)$ & & & \\
\hline Blank & 8022R01142 & $<5$ & $<5$ & $<5$ & \\
\hline Spike, \% Recovery & $8022 \mathrm{R} 01142$ & 82.45 & 64.24 & E32.76 & 64.51 \\
\hline CRM 354, \% Recovery (Acceptable Range) & $8022 \mathrm{R} 01142$ & $79.84(41-90)$ & & & \\
\hline Blank & $8022 \mathrm{H} 00322$ & $<5$ & $<5$ & $<5$ & \\
\hline Spike, \% Recovery & $8022 \mathrm{H} 00322$ & 101.30 & 78.22 & E26.55 & 81.63 \\
\hline CRM 354, \% Recovery (Acceptable Range) & $8022 \mathrm{H} 00322$ & $100.1(41-90)$ & & & \\
\hline Blank & $8022 \mathrm{R} 01141$ & $<5$ & $<5$ & $<5$ & \\
\hline Spike, \% Recovery & $8022 \mathrm{R} 01141$ & 78.45 & 60.47 & E30.01 & 71.23 \\
\hline CRM 354, \% Recovery (Acceptable Range) & $8022 \mathrm{R} 01141$ & $75.39(41-90)$ & & & \\
\hline Blank & 8022R00299 & $<5$ & $<5$ & $<5$ & \\
\hline Spike, \% Recovery & 8022R00299 & 100.68 & 90.5 & E12.76 & 90.12 \\
\hline CRM 354, \% Recovery (Acceptable Range) & $8022 \mathrm{R} 00299$ & $86.03(41-90)$ & & & \\
\hline Blank & $8022 \mathrm{R} 00285$ & $<5$ & $<5$ & $<5$ & \\
\hline Spike, \% Recovery & $8022 \mathrm{R} 00285$ & 100.08 & 85.73 & E19.87 & 87.38 \\
\hline CRM 354, \% Recovery (Acceptable Range) & $8022 R 00285$ & $94.66(41-90)$ & & & \\
\hline Blank & $8022 \mathrm{H} 00305$ & $<5$ & $<5$ & $<5$ & \\
\hline Spike, \% Recovery & $8022 \mathrm{H} 00305$ & 93.54 & 79.15 & E25.16 & 79.00 \\
\hline CRM 354, \% Recovery (Acceptable Range) & $8022 \mathrm{H} 00305$ & $79.94(41-90)$ & & & \\
\hline Blank & $8022 \mathrm{H} 00321$ & $<5$ & $<5$ & $<5$ & \\
\hline Spike, \% Recovery & $8022 \mathrm{H} 00321$ & 106.48 & 82.76 & E23.81 & 83.27 \\
\hline CRM 354, \% Recovery (Acceptable Range) & $8022 \mathrm{H} 00321$ & $83.00(41-90)$ & & & \\
\hline
\end{tabular}


MEDIAN SPIKE

RECOVERY (\%)

\begin{tabular}{|c|c|c|c|c|c|}
\hline Sample ID & Batch & Chrysene & Benzo (a) pyrene & Coronene & FOR BATC \\
\hline Blank & $8022 \mathrm{R} 01137$ & $2<5$ & $2<5$ & $\overline{<5}$ & \\
\hline Spike, \% Recovery & $8022 \mathrm{R} 01137$ & 71.12 & 66.69 & E57.20 & 68.12 \\
\hline CRM 354, \% Recovery (Acceptable Range) & $8022 \mathrm{R} 01137$ & $72.02(41-90)$ & & & \\
\hline Blank & 8022R01002 & $<5$ & $<5$ & $<5$ & \\
\hline Spike, \% Recovery & 8022R01002 & 96.65 & 98.99 & E13.88 & 92.39 \\
\hline CRM 354, \% Recovery (Acceptable Range) & 8022R01002 & $108.14(41-90)$ & & & \\
\hline Blank & 8022R01112 & $<5$ & $<5$ & $<5$ & \\
\hline Spike, \% Recovery & 8022R01112 & 70.41 & 67.20 & E38.58 & 64.69 \\
\hline CRM 354, \% Recovery (Acceptable Range) & 8022R01112 & $75.75(41-90)$ & & & \\
\hline Blank & 8022R01206 & $<5$ & $<5$ & $<5$ & \\
\hline Spike, \% Recovery & 8022R01206 & 88.91 & 64.87 & E20.74 & 65.24 \\
\hline CRM 354, \% Recovery (Acceptable Range) & 8022R01206 & $81.60(41-90)$ & & & \\
\hline Blank & 8022R01205 & $<5$ & $<5$ & $<5$ & \\
\hline Spike, \% Recovery & 8022R01205 & 86.57 & 62.24 & E17.84 & 63.57 \\
\hline CRM 354, \% Recovery (Acceptable Range) & 8022R01205 & $89.58(41-90)$ & & & \\
\hline Blank & 8022R01215 & $<5$ & $<5$ & $<5$ & \\
\hline Spike, \% Recovery & $8022 R 01215$ & 74.48 & 66.84 & E18.37 & 65.36 \\
\hline CRM 354, \% Recovery (Acceptable Range) & $8022 R 01215$ & $67.19(41-90)$ & & & \\
\hline Blank & 8022R01164 & $<5$ & $<5$ & $<5$ & \\
\hline Spike, \% Recovery & 8022R01164 & 75.63 & 46.31 & E27.30 & 64.24 \\
\hline CRM 354, \% Recovery (Acceptable Range) & $8022 R 01164$ & $76.64(41-90)$ & & & \\
\hline Blank & 8022R01234 & $<5$ & $<5$ & $<5$ & \\
\hline Spike, \% Recovery & 8022R01234 & 78.54 & E44.38 & E43.99 & 67.15 \\
\hline CRM 354, \% Recovery (Acceptable Range) & $8022 R 01234$ & & & & \\
\hline Blank & $8022 R 01235$ & $<5$ & $<5$ & $<5$ & \\
\hline Spike, \% Recovery & $8022 R 01235$ & 84.76 & 72.56 & E60.56 & 76.04 \\
\hline CRM 362, \% Recovery (Acceptable Range) & $8022 R 01235$ & $73.43(42-126)$ & $48.11(39-104)$ & & \\
\hline Blank & $8022 \mathrm{R} 01261$ & $<5$ & $<5$ & $<5$ & \\
\hline Spike, \% Recovery & $8022 R 01261$ & 79.81 & 67.52 & E54.50 & 69.48 \\
\hline
\end{tabular}


MEDIAN SPIKE

RECOVERY (\%)

Sample ID

Batch

Chrysene Benzo (a) pyrene

Coronene

FOR BATCH

CRM 362, \% Recovery (Acceptable Range)

8022R01261

$52.82(39-104)$

Blank

Spike, \% Recovery

8022R01243

$8022 \mathrm{R} 01243$

$<5$

71.31

$<5$

$8022 \mathrm{R} 01243$

$67.79(42-126)$

59.98

$<5$

CRM 362, \% Recovery (Acceptable Range)

$8022 R 02192$

\section{Blank}

Spike, \% Recovery

$8022 R 02192$

$<5$

76.93

$52.20(39-104)$

E29.45

67.87

CRM 362, \% Recovery (Acceptable Range)

$<5$

61.30

E42.13

$42.86(39-104)$ 
Table B4. Polycyclic aromatic hydrocarbon duplicate samples.

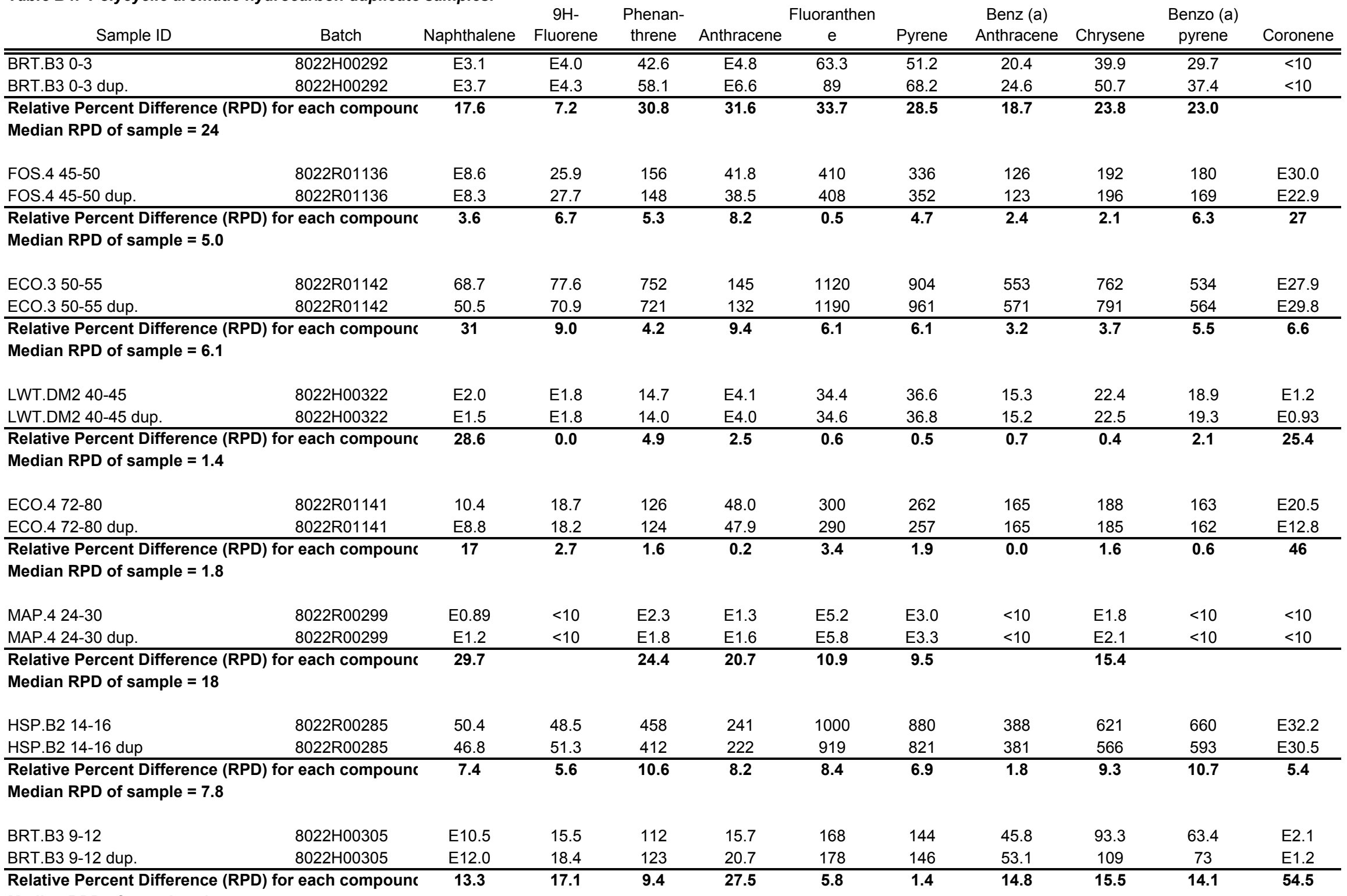

Median RPD of sample $=14$ 
9H- Phenan-

Fluoranthen

Benz (a) Benzo (a)

Batch Naphthalene Fluorene threne Anthracene

Pyrene Anthracene Chrysene pyrene

Coronene

FOS.2 100-110

$8022 \mathrm{R} 01137$

8022R01137

E4.3 11.1

FOS.2 100-110 dup.

E4.7 12.4

12.4

13.9

E9.

$\begin{array}{lll}39.6 & 39.3 & 12.9\end{array}$

\begin{tabular}{cc}
43.3 & 14.4 \\
\hline 9.7 & 11
\end{tabular}

11

\begin{tabular}{lll}
22.9 & 16.4 & E7.0 \\
25.1 & 18.6 & E8.3 \\
\hline
\end{tabular}

Median RPD of sample $=11$

MYS.B2 28-30

$8022 \mathrm{R} 01002$

\begin{tabular}{ll}
530 & 452 \\
509 & 366 \\
\hline
\end{tabular}

MYS.B2 28-30 dup $8022 R 01002$

$4.0 \quad 21.0$

\begin{tabular}{ccccccccc}
452 & 3800 & 2130 & 10800 & 10100 & 4630 & 6460 & 6770 & E1140 \\
366 & 3640 & 2070 & 9840 & 9460 & 4370 & 5910 & 6350 & E1040 \\
\hline $\mathbf{2 1 . 0}$ & $\mathbf{4 . 3}$ & $\mathbf{2 . 9}$ & $\mathbf{9 . 3}$ & $\mathbf{6 . 5}$ & $\mathbf{5 . 8}$ & $\mathbf{8 . 9}$ & $\mathbf{6 . 4}$ & $\mathbf{9 . 2}$
\end{tabular}

Relative Percent Difference (RPD) for each compoun

Median RPD of sample $=6.5$

CMO. $130-35$

8022R01206

$8022 R 01206$

$17.8 \quad 57.6$

$\begin{array}{cc}17.0 & 63.4 \\ \mathbf{4 . 6} & \mathbf{1 0}\end{array}$

\begin{tabular}{ll}
537 & 131 \\
\hline 10 & 484
\end{tabular}

CMO $130-35$ dup.

for each compounc

\begin{tabular}{ll}
484 & 134 \\
\hline 10 & 2.3
\end{tabular}

1020

802

\begin{tabular}{lcccc}
754 & 889 & 629 & E52.7 \\
\hline 6 & 495 & 731 & 550 & E31.8 \\
\hline
\end{tabular}

Median RPD of sample $=14$

Town Lake in Austin, TX

$18.2 \quad 44.2$

146

$022 \mathrm{R} 01215$

$18.3 \quad 44.9$

\begin{tabular}{rrrrrrrr}
640 & 146 & 2690 & 2120 & 1040 & 1720 & 1400 & E93.0 \\
\hline
\end{tabular}

Relative Percent Difference (RPD) for each compounc

0.5

1.6

$3.5 \quad 3.4$

2780

Median RPD of sample $=3.7$

CMO. $145-50$

CMO.1 45-50 dup.

$8022 \mathrm{R} 01234$

$21.1 \quad 71$.

$21.5 \quad 69.7$

69.7

\begin{tabular}{ll}
745 & 188 \\
715 & 183 \\
\hline
\end{tabular}

$188 \quad E 2020$

E1900 E1540

$\begin{array}{lllll}730 & 1120 & \text { E800 } & \text { E119 }\end{array}$

Relative Percent Difference (RPD) for each compounc

(ample $=4.1$

Busse Lake nr Chicago, IL

$8022 \mathrm{R} 01235$

\begin{tabular}{ccc}
67.5 & 274 & 1660 \\
70 & 287 & 1820 \\
\hline
\end{tabular}

Busse Lake nr Chicago, IL dup $8022 \mathrm{R} 01235$

$1820 \quad 487$

$7.4 \quad 4.1$

$\begin{array}{ccc}11070 & E 798 & E 74.2 \\ \mathbf{4 . 6} & \mathbf{0 . 3} & \mathbf{4 6}\end{array}$

$3.6 \quad 4$

Median RPD of sample $=4.1$

Lake in the Hills $\mathrm{nr}$ Chicago, IL

Lake in the Hills nr Chic

E11.2

63.3

Relative Percent Difference (RPD) for each compounc

E9.5

60.1

$6 \quad 9.2 \quad 10.8$

$9970 \quad 6860$

$\begin{array}{llllll}10500 & 7090 & 2820 & 4570 & 3540 & \text { E624 }\end{array}$

Median RPD of sample $=16$

Lake Austin, TX

$8022 \mathrm{R} 02192$

E1.7

E2.5

Relative Percent Difference (RPD) for each compounc

$\begin{array}{lll} & \text { E2.5 } & \text { E4 } \\ 11 & \mathbf{2 7} & \end{array}$

E7.1 E8.1

E8.1 27.1 


\begin{tabular}{|c|c|c|c|c|c|c|c|c|c|c|}
\hline MIL.B3 10-15 & E8.6 & E9.7 & 31.3 & E8.0 & 50.6 & 36.2 & 16.2 & 23.9 & 23.8 & E10.8 \\
\hline MIL.B3 10-15 dup. & E8.4 & E9.5 & 27.9 & E7.1 & 48.6 & 34.4 & 16.5 & 22.5 & 21.4 & E7.5 \\
\hline $\begin{array}{l}\text { Relative Percent Difference (RPD) for each compounc } \\
\text { Median RPD of sample }=8.6\end{array}$ & 2.4 & 2.1 & 11.5 & 11.9 & 4.0 & 5.1 & 1.8 & 6.0 & 10.6 & 36.1 \\
\hline $\begin{array}{l}\text { MEDIAN RPD FOR EACH COMPOUND: } \\
\text { MEDIAN RPD FOR ALL BATCHES AND COMPOUNDS }\end{array}$ & 8.9 & 6.2 & 9.2 & 9.4 & 6.1 & 6.5 & 4.9 & 6.0 & 8.5 & 17.3 \\
\hline
\end{tabular}


Table B5. Sample groups for organics analyses and duplicate sample
OC set number Samples grouped for analysis

Sites 2 and 6 (03/08/01); Site 4 (03/09/01); Big Fossil (05/04/01): FOS. 4 20-25, 45-50, 70-75

ECO.15-10, 10-15, 20-25, 65-70, 75-80, 90-97; ECO.3

ECO- 55 ;
ECO.4 0-8

MYS.B2 12-14, 16-18, and 34-36

ECO. 1 0-5, 30-35, 40-45, 55-60; ECO.3 0-5, 25-30; ECO. 4 40-48, $72-80$

$0-2$ and $20-22$

HSP.B2 4-6, 6-8, 8-10, 12-14, 14-16, 14-16 lab. dup., 16 18 , 18-20, and 22-24: HSP B3 0-6 and 24-30: HSP. $10-$ MYS.B2 $0-2,2-4,4-6,6-8$, and $8-10$

MYS. $20-8,14-19$, and 25-30

FOS.2 0-10, 50-60, 100-110; FOS.4 0-5, 5-10, 10-15, $30-35,55-60,85-90,100-105$; FOS $50-10,40-50$

MYS.B2 20-22, 24-26, 28-30, and 28-30 lab. dup.

Sites $1,2,3,4,5$, and 6 (10/15/00); Site 1 (03/08/01) Aberjona River Composite \#1 and \#2 (03/27/2001): Aberjona River Discreet \#1 (03/22/2001), \#2 (03/28/2001); Mill River (03/28/2001)

CMO.1 10-15, 30-35; Charles River (05/30/2001); MiI River (05/30/2001)

(10.1 0-5, 5-10, 20-25, 60-65, 75-80, 90-95: CMO $30-$ 10, 40-50, 90-100; CMO. $50-10,20-30,40-50$

Site 5 (05/04/01); Site 5 duplicate (05/04/01); Sycamore \#2 and 3 (05/04/01); Sycamore \#4\&5 (05/05/01); Sites

Sycamore \#6\&7 (05/05/01)

Big Fossil (08/11/01); CMO.1 45-50

Site 5 (08/16/01); Site 5 duplicate (08/16/01); Sycamo 2,3 , and 4 (08/16/01); Site 1 (08/17/01); Echo Lake Inflow (08/17/01): Sycamore \#4 (08/30/01)

Sycamore \#1, 2, 3, and 5 (08/30/01); Lake Como Inflow (08/30/01, 09/20/01); Big Fossi (09//8/101, 10/11/01) osdic Lake Inflow (09/18/01, Fosdic Lake Inflow (12/06/01)

Fosdic Lake Inflow (08/11/01); Lake Como Inflow $(11 / 09 / 01,01 / 23 / 02)$

Lake Como Inflow (06/28/02)

Aberjona River Composite (08/26/2002)

ALL SETS IN STUDY percent differences.

PAH batch Type of duplicate(s), $\begin{gathered}\text { Type of spike, } \\ \text { range and median }\end{gathered}$

number range and median percent recovery

$\begin{array}{lll}8022 \mathrm{H} 00292 \text { OC: No duplicate } & \text { OC: } 85-202 \%, 124 \% \\ \text { PAH: } 72-34 \%, 24 \% & \text { PAH: } 7-96 \%, 25 \%\end{array}$ РAH: $0.5-27 \%, 50 \%$ PAH: $43-92 \%, 72 \%$ $8022 \mathrm{R} 01142$ OC: $0.0-33 \%, 17 \% \quad$ OC: $45-139 \%, 71 \%$ PAH: $3.2-31 \%, 6.1 \% \quad$ PAH: $33-82 \%, 65 \%$ $8022 \mathrm{H} 00322$ OC: No duplicate $\quad$ OC: $85-204 \%, 121 \%$ PAH: $0.0-29 \%, 1.4 \%$ PAH: $27-101 \%, 82 \%$ 3022R01141 OC: $0.0-7.4 \%, 5.1 \%$ OC: $54-131 \%, 81 \%$ PAH: $0.0-46 \%, 1.8 \%$ PAH: $30-78 \%, 71 \%$ 8022R00299 OC: No duplicate $\quad$ OC: $56-126 \%, 81 \%$ 8022 R00285 OC: $1.1-12 \%, 5.7 \%$ OC: $16-138 \%, 96 \%$ PAH: $1.8-11 \%, 7.8 \%$ PAH: $20-104 \%, 87 \%$ $8022 \mathrm{H} 00305$ OC: No duplicate OC: $30-111 \%, 87 \%$ PAH: $1.4-54 \%, 14 \%$ PAH: $25-94 \%, 79 \%$ 3022H00321 OC: No duplicate OC: $37-131 \%, 103 \%$ PAH: $24-106 \%, 83 \%$ 8022 R01137 OC: No detections $\quad$ OC: $61-118 \%, 89 \%$ PAH: $7.6-17 \%, 11 \%$ PAH: $54-81 \%, 68 \%$ 8022R01002 OC: $2.2-18 \%, 7.7 \%$ OC: $93-185 \%, 131 \%$ 8022R01112 OC. N.9-2plicate PAH: No duplicate PAH: $39-73 \%, 65 \%$

200120107 8022R01206 OC: $0.0-17 \%, 6.1 \%$; OC: $59-145 \%, 88 \%$ 8022R01205 OC: No duplicate OC: $65-143 \%, 83 \%$ PAH: Ruined in PAH: $18-87 \%, 64 \%$

200121505 8022R01215 OC: $16-78 \%, 38 \% \quad$ OC: $66-138 \%, 98 \%$

200122506 PAH: $0.5-13 \%, 3.7 \%$ PAH: $18-74 \%, 65 \%$ PAH: No duplicate PAH: $27-76 \%, 64 \%$ $200128405 \quad 8022 \mathrm{R} 01234$ OC: $2.7-55 \%, 6.1 \%$; OC: $65-244 \%, 112 \%$

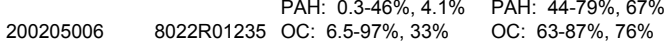
PAH: $1.4-11 \%, 4.1 \%$ PAH: $61-97 \%, 76 \%$ 200207106 8022R01261 OC: No duplicate OC: $66-94 \%, 88 \%$

$200207706 \quad$ PAH: No duplicate PAH: $55-82 \%, 69 \%$ $8022 R 01243$ OC: $2.1-129 \%, 4.9 \%$ OC: $61-97 \%, 84 \%$ $8022 \mathrm{R} 01235$ OC: $4.5 \% \quad$ OC: $52-94 \%, 78 \%$ PAH: $1.4-11 \%, 4.1 \%$ PAH: $61-97 \%, 76 \%$ $8022 \mathrm{R} 02192$ OC: $19 \% \quad$ OC: $70-118 \%, 89 \%$ 8022 R02199 PA: $28.5-50 \%, 29 \%$ PAH: $42-77 \%, 70 \%$ OC: $28-34 \%, 31 \%$ OC: $75-98 \%, 88 \%$ OC. $0.0-129 \%, 8.5 \%$ OC: $16-244 \%, 87 \%$ PAH: $0.0-55 \%, 8.2 \%$ PAH: $7-106 \%, 72 \%$ 
Table B6. Major and trace element quality control samples.

\begin{tabular}{|c|c|c|c|c|c|c|c|c|c|c|c|c|c|c|}
\hline Sample ID & Job no. or Core & Method & $\mathrm{Al}, \mathrm{ppm}$ & $\mathrm{Ca}, \mathrm{ppm}$ & $\mathrm{Fe}, \mathrm{ppm}$ & $\mathrm{K}, \mathrm{ppm}$ & $\mathrm{Mg}, \mathrm{ppm}$ & $\mathrm{Na}, \mathrm{ppm}$ & $\mathrm{P}, \mathrm{ppm}$ & Ti, ppm & As, ppm & $\mathrm{Ba}, \mathrm{ppm}$ & $\mathrm{Be}, \mathrm{ppm}$ & Cd, ppm \\
\hline MAG-1 laboratory & MYS.B2 core & ICPIAES & 83500 & 9600 & 47300 & 29800 & 17700 & 28900 & 720 & 4600 & 9.55 & 474.50 & & 0.22 \\
\hline MAG-1 literature & MYS.B2 core & ICP/AES & 86660 & 9790 & 47600 & 29500 & 18090 & 28400 & 711 & 4500 & 9.2 & 479 & & 0.20 \\
\hline RPD for each element $=$ & & & 3.7 & 2.0 & 0.6 & 1.0 & 2.2 & 1.7 & 1.3 & 2.2 & 3.7 & 0.9 & & 6.2 \\
\hline Median RPD for SRM = & 1.9 & & & & & & & & & & & & & \\
\hline NIST 2704 laboratory & MYS.B2 core & ICP/AES & 62100 & 26100 & 40433 & 19667 & 12067 & 5600 & 937 & 4667 & 22.50 & 411.00 & & 3.41 \\
\hline NIST 2704 literature & MYS.B2 core & ICP/AES & 61090 & 26000 & 41100 & 20000 & 12000 & 5470 & 1000 & 4580 & 23.4 & 414 & & 3.45 \\
\hline RPD for each element $=$ & & & 1.6 & 0.4 & 1.6 & 1.7 & 0.6 & 2.3 & 6.5 & 1.9 & 3.9 & 0.7 & & 1.1 \\
\hline Median RPD for SRM = & 1.2 & & & & & & & & & & & & & \\
\hline SCo-1 laboratory & MYS.B2 core & ICP/AES & 72250 & 18450 & 34900 & 22450 & 16250 & 6800 & 935 & 3900 & 12.20 & 576.00 & & 0.16 \\
\hline SCo-1 literature & MYS.B2 core & ICP/AES & 72370 & 18700 & 35900 & 23000 & 16400 & 6670 & 899 & 3760 & 12.4 & 570 & & 0.14 \\
\hline RPD for each element $=$ & & & 0.2 & 1.3 & 2.8 & 2.4 & 0.9 & 1.9 & 3.9 & 3.7 & 1.6 & 1.0 & & 10.2 \\
\hline Median RPD for SRM = & 1.6 & & & & & & & & & & & & & \\
\hline GSD-8 laboratory & MYS.B2 core & ICP/AES & 41650 & 1850 & 15650 & 23600 & 1400 & 3400 & 125 & 3650 & 2.70 & 485.50 & & 0.09 \\
\hline GSD-8 literature & MYS.B2 core & ICPIAES & 40800 & 1790 & 15380 & 23500 & 1510 & 3490 & 130 & 3660 & 2.4 & 480 & & 0.08 \\
\hline RPD for each element $=$ & & & 2.1 & 3.3 & 1.7 & 0.4 & 7.6 & 2.6 & 4.3 & 0.3 & 11.8 & 1.1 & & 7.1 \\
\hline Median RPD for SRM = & 2.6 & & & & & & & & & & & & & \\
\hline MESS-2 laboratory & MYS.B2 core & ICP/AES & 84850 & 14950 & 43750 & 26300 & 12650 & 14250 & 1200 & 4000 & 19.10 & 796.50 & & 0.23 \\
\hline MESS-2 literature (no symbols) & MYS.B2 core & ICP/AES & 85700 & & 43500 & & & & 1200 & & 20.7 & & & 0.24 \\
\hline MESS-2 literature & MYS.B2 core & ICP/AES & $85700 \pm 2600$ & & $43500 \pm 2200$ & & & & $1200 \pm 100$ & & $20.7 \pm 0.8$ & & & $0.24 \pm 0.01$ \\
\hline $\begin{array}{r}\text { RPD for each element }= \\
\text { Median RPD for SRM }=\end{array}$ & 1.6 & & 1.0 & & 0.6 & & & & 0.0 & & 8.0 & & & 4.3 \\
\hline MAG-1 laboratory & HSP. 1 core & ICP/AES & 83800 & 10000 & 47400 & 30500 & 17700 & 27900 & 770 & 4500 & 9.8 & 468 & & 0.19 \\
\hline MAG-1 literature & HSP.1 core & ICPIAES & 86660 & 9790 & 47600 & 29500 & 18090 & 28400 & 711 & 4500 & 9.2 & 479 & & 0.20 \\
\hline RPD for each element $=$ & & & 3.4 & 2.1 & 0.4 & 3.3 & 2.2 & 1.8 & 8.0 & 0.0 & 6.3 & 2.3 & & 6.1 \\
\hline Median RPD for SRM = & 2.0 & & & & & & & & & & & & & \\
\hline NIST 2704 laboratory & HSP.1 core & ICP/AES & 61100 & 26700 & 40800 & 19700 & 11900 & 5300 & 990 & 4700 & 23.5 & 416 & & 3.20 \\
\hline NIST 2704 literature & HSP.1 core & ICPIAES & 61090 & 26000 & 41100 & 20000 & 12000 & 5470 & 1000 & 4580 & 23.4 & 414 & & 3.45 \\
\hline RPD for each element $=$ & & & 0.0 & 2.7 & 0.7 & 1.5 & 0.8 & 3.2 & 1.0 & 2.6 & 0.4 & 0.5 & & 7.5 \\
\hline Median RPD for SRM $=$ & 2.6 & & & & & & & & & & & & & \\
\hline SCo-1 laboratory & HSP.1 core & ICP/AES & 73800 & 18900 & 35800 & 22600 & 16800 & 6700 & 860 & 3900 & 11.6 & 607 & & 0.14 \\
\hline SCo-1 literature & HSP. 1 core & ICPIAES & 72370 & 18700 & 35900 & 23000 & 16400 & 6670 & 899 & 3760 & 12.4 & 570 & & 0.14 \\
\hline $\begin{array}{r}\text { RPD for each element }= \\
\text { Median RPD for SRM }=\end{array}$ & 2.3 & & 2.0 & 1.1 & 0.3 & 1.8 & 2.4 & 0.4 & 4.4 & 3.7 & 6.7 & 6.3 & & 0.0 \\
\hline GSD-8 laboratory & HSP. 1 core & ICP/AES & 40200 & 1800 & 15400 & 24100 & 1600 & 3500 & 110 & 3600 & 2.6 & 476 & & 0.08 \\
\hline GSD-8 literature & HSP.1 core & ICP/AES & 40800 & 1790 & 15380 & 23500 & 1510 & 3490 & 130 & 3660 & 2.4 & 480 & & 0.08 \\
\hline $\begin{array}{r}\text { RPD for each element }= \\
\text { Median RPD for SRM }=\end{array}$ & 2.3 & & 1.5 & 0.6 & 0.1 & 2.5 & 5.8 & 0.3 & 16.7 & 1.7 & 8.0 & 0.8 & & 1.2 \\
\hline MESS-2 laboratory & HSP. 1 core & ICPIAES & 88700 & 14000 & 44700 & 26100 & 12300 & 14100 & 1100 & 4000 & 20.8 & 800 & & 0.23 \\
\hline MESS-2 literature (no symbols) & HSP. 1 core & ICP/AES & 85700 & & 43500 & & & & 1200 & & 20.7 & & & 0.24 \\
\hline MESS-2 literature & HSP.1 core & ICP/AES & $85700 \pm 2600$ & & $43500 \pm 2200$ & & & & $1200 \pm 100$ & & $20.7 \pm 0.8$ & & & $0.24 \pm 0.01$ \\
\hline $\begin{array}{r}\text { RPD for each element }= \\
\text { Median RPD for SRM }=\end{array}$ & 1.2 & & 3.4 & & 2.7 & & & & 8.7 & & 0.5 & & & 4.3 \\
\hline MAG-1 laboratory & HSP.B2 core & ICP/AES & 51600 & 9800 & 48500 & 29500 & 18300 & 28800 & 660 & 4600 & 9.3 & 496 & & 0.21 \\
\hline MAG-1 literature & HSP.B2 core & ICP/AES & 86660 & 9790 & 47600 & 29500 & 18090 & 28400 & 711 & 4500 & 9.2 & 479 & & 0.20 \\
\hline RPD for each element $=$ & & & 50.7 & 0.1 & 1.9 & 0.0 & 1.2 & 1.4 & 7.4 & 2.2 & 1.1 & 3.5 & & 3.9 \\
\hline
\end{tabular}

Median RPD for SRM =

1.9 


\begin{tabular}{|c|c|c|c|c|c|c|c|c|c|c|c|c|c|c|}
\hline Sample ID & Job no. or Core & Method & $\mathrm{Al}, \mathrm{ppm}$ & $\mathrm{Ca}, \mathrm{ppm}$ & $\mathrm{Fe}, \mathrm{ppm}$ & $\mathrm{K}, \mathrm{ppm}$ & $\mathrm{Mg}, \mathrm{ppm}$ & $\mathrm{Na}, \mathrm{ppm}$ & $\mathrm{P}, \mathrm{ppm}$ & $\mathrm{Ti}, \mathrm{ppm}$ & As, ppm & Ba, ppm & $\mathrm{Be}, \mathrm{ppm}$ & $\mathrm{Cd}, \mathrm{ppm}$ \\
\hline NIST 2704 laboratory & "HSP.B2 core & $\overline{~ I C P / A E S}$ & $=62200$ & 26100 & $4 \quad 42400$ & 20200 & $=11900$ & 25500 & 9950 & $=4900$ & 23.9 & 4421 & & $=3.23$ \\
\hline NIST 2704 literature & HSP.B2 core & ICP/AES & 61090 & 26000 & 41100 & 20000 & 12000 & 5470 & 1000 & 4580 & 23.4 & 414 & & 3.45 \\
\hline RPD for each element $=$ & & & 1.8 & 0.4 & 3.1 & 1.0 & 0.8 & 0.5 & 5.1 & 6.8 & 2.1 & 1.7 & & 6.6 \\
\hline Median RPD for SRM = & 1.8 & & & & & & & & & & & & & \\
\hline SCo-1 laboratory & HSP.B2 core & ICP/AES & 41600 & 18700 & 35600 & 22800 & 17000 & 6900 & 890 & 3600 & 12.2 & 577 & & 0.13 \\
\hline SCo-1 literature & HSP.B2 core & ICP/AES & 72370 & 18700 & 35900 & 23000 & 16400 & 6670 & 899 & 3760 & 12.4 & 570 & & 0.14 \\
\hline RPD for each element $=$ & & & 54.0 & 0.0 & 0.8 & 0.9 & 3.6 & 3.4 & 1.0 & 4.3 & 1.6 & 1.2 & & 7.4 \\
\hline Median RPD for SRM = & 1.5 & & & & & & & & & & & & & \\
\hline GSD-8 laboratory & HSP.B2 core & ICP/AES & 41700 & 1700 & 15300 & 23800 & 1500 & 3600 & 110 & 3900 & 3.3 & 475 & & 0.07 \\
\hline GSD-8 literature & HSP.B2 core & ICP/AES & 40800 & 1790 & 15380 & 23500 & 1510 & 3490 & 130 & 3660 & 2.4 & 480 & & 0.08 \\
\hline $\begin{array}{r}\text { RPD for each element }= \\
\text { Median RPD for SRM }=\end{array}$ & 2.4 & & 2.2 & 5.2 & 0.5 & 1.3 & 0.7 & 3.1 & 16.7 & 6.3 & 31.6 & 1.0 & & 17.4 \\
\hline MESS-2 laboratory & HSP.B2 core & ICP/AES & 83900 & 14700 & 43100 & 25900 & 13000 & 14100 & 1200 & 4000 & $\mathrm{n} / \mathrm{a}$ & 794 & & 0.23 \\
\hline MESS-2 literature (no symbols) & HSP.B2 core & ICPIAES & 85700 & & 43500 & & & & 1200 & & 20.7 & & & 0.24 \\
\hline MESS-2 literature & HSP.B2 core & ICP/AES & $85700 \pm 2600$ & & $43500 \pm 2200$ & & & & $1200 \pm 100$ & & $20.7 \pm 0.8$ & & & $0.24 \pm 0.01$ \\
\hline $\begin{array}{l}\text { RPD for each element }= \\
\text { Median RPD for SRM }=\end{array}$ & 1.4 & & 2.1 & & 0.9 & & & & 0.0 & & & & & 4.3 \\
\hline MAG-1 laboratory & HSP.B3 core & ICP/AES & 86200 & 10000 & 49500 & 30400 & 17500 & 29100 & 710 & 4500 & 9.3 & 466 & & 0.17 \\
\hline MAG-1 literature & HSP.B3 core & ICP/AES & 86660 & 9790 & 47600 & 29500 & 18090 & 28400 & 711 & 4500 & 9.2 & 479 & & 0.20 \\
\hline RPD for each element $=$ & & & 0.5 & 2.1 & 3.9 & 3.0 & 3.3 & 2.4 & 0.1 & 0.0 & 1.1 & 2.8 & & 17.2 \\
\hline Median RPD for SRM = & 2.6 & & & & & & & & & & & & & \\
\hline NIST 2704 laboratory & HSP.B3 core & ICP/AES & 62000 & 25100 & 40100 & 20400 & 11300 & 5500 & 940 & 4500 & 24 & 415 & & 3.27 \\
\hline NIST 2704 literature & HSP.B3 core & ICP/AES & 61090 & 26000 & 41100 & 20000 & 12000 & 5470 & 1000 & 4580 & 23.4 & 414 & & 3.45 \\
\hline RPD for each element $=$ & & & 1.5 & 3.5 & 2.5 & 2.0 & 6.0 & 0.5 & 6.2 & 1.8 & 2.5 & 0.2 & & 5.4 \\
\hline Median RPD for SRM $=$ & 2.5 & & & & & & & & & & & & & \\
\hline SCo-1 laboratory & HSP.B3 core & ICP/AES & 72100 & 18800 & 36400 & 22700 & 16600 & 6800 & 810 & 3700 & $\mathrm{n} / \mathrm{a}$ & 567 & & 0.10 \\
\hline SCo-1 literature & HSP.B3 core & ICP/AES & 72370 & 18700 & 35900 & 23000 & 16400 & 6670 & 899 & 3760 & 12.4 & 570 & & 0.14 \\
\hline RPD for each element $=$ & & & 0.4 & 0.5 & 1.4 & 1.3 & 1.2 & 1.9 & 10.4 & 1.6 & & 0.5 & & 33.3 \\
\hline Median RPD for SRM = & 1.7 & & & & & & & & & & & & & \\
\hline GSD-8 laboratory & HSP.B3 core & ICP/AES & 42300 & 1800 & 15800 & 23600 & 1500 & 3500 & 110 & 3700 & 2.7 & 495 & & 0.08 \\
\hline GSD-8 literature & HSP.B3 core & ICP/AES & 40800 & 1790 & 15380 & 23500 & 1510 & 3490 & 130 & 3660 & 2.4 & 480 & & 0.08 \\
\hline RPD for each element $=$ & & & 3.6 & 0.6 & 2.7 & 0.4 & 0.7 & 0.3 & 16.7 & 1.1 & 11.8 & 3.1 & & 2.5 \\
\hline Median RPD for SRM = & 2.7 & & & & & & & & & & & & & \\
\hline MESS-2 laboratory & HSP.B3 core & ICP/AES & 84200 & 14900 & 41900 & 25800 & 13200 & 13600 & 1100 & 4100 & 19.9 & 784 & & 0.23 \\
\hline MESS-2 literature (no symbols) & HSP.B3 core & ICP/AES & 85700 & & 43500 & & & & 1200 & & 20.7 & & & 0.24 \\
\hline MESS-2 literature & HSP.B3 core & ICP/AES & $85700 \pm 2600$ & & $43500 \pm 2200$ & & & & $1200 \pm 100$ & & $20.7 \pm 0.8$ & & & $0.24 \pm 0.01$ \\
\hline RPD for each element $=$ & & & 1.8 & & 3.7 & & & & 8.7 & & 3.9 & & & 4.3 \\
\hline Median RPD for SRM = & 3.3 & & & & & & & & & & & & & \\
\hline \multicolumn{15}{|c|}{ ICP/AES METHOD } \\
\hline Median RPD for MAG-1 & for each element: & & 3.5 & 2.0 & 1.3 & 2.0 & 2.2 & 1.8 & 4.3 & 1.1 & 2.4 & 2.5 & & 6.2 \\
\hline Median RPD for NIST 2704 & for each element: & & 1.6 & 1.5 & 2.0 & 1.6 & 0.8 & 1.4 & 5.7 & 2.2 & 2.3 & 0.6 & & 6.0 \\
\hline Median RPD for SCo-1 & for each element: & & 1.2 & 0.8 & 1.1 & 1.5 & 1.8 & 1.9 & 4.2 & 3.7 & 1.6 & 1.1 & & 8.8 \\
\hline Median RPD for GSD-8 & for each element: & & 2.1 & 1.9 & 1.1 & 0.8 & 3.2 & 1.4 & 16.7 & 1.4 & 11.8 & 1.1 & & 4.8 \\
\hline $\begin{array}{r}\text { Median RPD for MESS-2 } \\
\text { MEDIAN RPD FOR ALL SRMs }=1.9\end{array}$ & for each element: & & 1.9 & & 1.8 & & & & 4.3 & & 3.9 & & & 4.3 \\
\hline MAG-1 laboratory & 3186 and 3188 & ICP/MS & 80600 & 9750 & 47300 & 29200 & 18000 & 28400 & 696 & 4050 & 9.26 & 513 & 3.34 & 0.290 \\
\hline MAG-1 literature & 3186 and 3188 & ICP/MS & 86660 & 9790 & 47600 & 29500 & 18090 & 28400 & 711 & 4500 & 9.2 & 479 & 3.20 & 0.20 \\
\hline
\end{tabular}




\begin{tabular}{|c|c|c|c|c|c|c|c|c|c|c|c|c|c|c|}
\hline Sample ID & Job no. or Core & Method & $\mathrm{Al}, \mathrm{ppm}$ & $\mathrm{Ca}, \mathrm{ppm}$ & $\mathrm{Fe}, \mathrm{ppm}$ & $\mathrm{K}, \mathrm{ppm}$ & $\mathrm{Mg}, \mathrm{ppm}$ & $\mathrm{Na}, \mathrm{ppm}$ & $\mathrm{P}, \mathrm{ppm}$ & $\mathrm{Ti}, \mathrm{ppm}$ & As, ppm & $\mathrm{Ba}, \mathrm{ppm}$ & $\mathrm{Be}, \mathrm{ppm}$ & $\mathrm{Cd}, \mathrm{ppm}$ \\
\hline RPD for each element $=$ & & & 7.7 .2 & 0.4 & 0.6 & 101.0 & 0.5 & 0.0 & 2.1 & 10.5 & $\begin{array}{c}0.7 \\
0.7\end{array}$ & 6.9 & 44.3 & $\begin{array}{ll}35.8 \\
\end{array}$ \\
\hline Median RPD for SRM = & 3.4 & & & & & & & & & & & & & \\
\hline NIST 2704 laboratory & 3186 and 3188 & ICP/MS & 58400 & 25600 & 41000 & 19900 & 12000 & 5630 & 989 & 3000 & 22.3 & 428 & 1.89 & 3.50 \\
\hline NIST 2704 literature & 3186 and 3188 & ICP/MS & 61090 & 26000 & 41100 & 20000 & 12000 & 5470 & 1000 & 4580 & 23.4 & 414 & & 3.45 \\
\hline RPD for each element $=$ & & & 4.5 & 1.6 & 0.2 & 0.5 & 0.0 & 2.9 & 1.1 & 41.7 & 4.8 & 3.3 & & 1.4 \\
\hline Median RPD for SRM = & 3.0 & & & & & & & & & & & & & \\
\hline SCo-1 laboratory & 3186 and 3188 & ICP/MS & 68300 & 18100 & 35800 & 22300 & 15800 & 6540 & 900 & 3620 & 13.0 & 593 & 1.81 & 0.147 \\
\hline SCo-1 literature & 3186 and 3188 & ICP/MS & 72370 & 18700 & 35900 & 23000 & 16400 & 6670 & 899 & 3760 & 12.4 & 570 & 1.84 & 0.14 \\
\hline RPD for each element $=$ & & & 5.8 & 3.3 & 0.3 & 3.1 & 3.7 & 2.0 & 0.1 & 3.8 & 4.7 & 4.0 & 1.6 & 4.9 \\
\hline Median RPD for SRM = & 3.8 & & & & & & & & & & & & & \\
\hline GSD-8 laboratory & 3186 and 3188 & ICP/MS & 38900 & 2030 & 12600 & 24600 & 1480 & 3090 & 126 & 3940 & 2.48 & 458 & 2.07 & 0.028 \\
\hline GSD-8 literature & 3186 and 3188 & ICP/MS & 40800 & 1790 & 15380 & 23500 & 1510 & 3490 & 130 & 3660 & 2.4 & 480 & 2.00 & 0.08 \\
\hline RPD for each element $=$ & & & 4.8 & 12.6 & 19.9 & 4.6 & 2.0 & 12.2 & 3.1 & 7.4 & 3.3 & 4.7 & 3.4 & 97.2 \\
\hline Median RPD for SRM = & 5.6 & & & & & & & & & & & & & \\
\hline MAG-1 laboratory & 3201 and 3202 & ICP/MS & 85400 & 9840 & 48500 & 30600 & 18100 & 28000 & 746 & 4290 & 9.81 & 513 & 3.24 & 0.340 \\
\hline MAG-1 literature & 3201 and 3202 & ICP/MS & 86660 & 9790 & 47600 & 29500 & 18090 & 28400 & 711 & 4500 & 9.2 & 479 & 3.20 & 0.20 \\
\hline RPD for each element $=$ & & & 1.5 & 0.5 & 1.9 & 3.7 & 0.1 & 1.4 & 4.8 & 4.8 & 6.4 & 6.9 & 1.2 & 50.9 \\
\hline Median RPD for SRM $=$ & 3.7 & & & & & & & & & & & & & \\
\hline NIST 2704 laboratory & 3201 and 3202 & ICP/MS & 60200 & 24400 & 40700 & 19100 & 12600 & 5730 & 1000 & 3000 & 22.2 & 428 & 2.08 & 3.78 \\
\hline NIST 2704 literature & 3201 and 3202 & ICP/MS & 61090 & 26000 & 41100 & 20000 & 12000 & 5470 & 1000 & 4580 & 23.4 & 414 & & 3.45 \\
\hline RPD for each element $=$ & & & 1.5 & 6.3 & 1.0 & 4.6 & 4.9 & 4.6 & 0.0 & 41.7 & 5.3 & 3.3 & & 9.1 \\
\hline Median RPD for SRM = & 4.6 & & & & & & & & & & & & & \\
\hline SCo-1 laboratory & 3201 and 3202 & ICP/MS & 72700 & 17400 & 37200 & 22300 & 17400 & 6840 & 934 & 3940 & 12.6 & 614 & 1.98 & 0.173 \\
\hline SCo-1 literature & 3201 and 3202 & ICP/MS & 72370 & 18700 & 35900 & 23000 & 16400 & 6670 & 899 & 3760 & 12.4 & 570 & 1.84 & 0.14 \\
\hline RPD for each element $=$ & & & 0.5 & 7.2 & 3.6 & 3.1 & 5.9 & 2.5 & 3.8 & 4.7 & 1.6 & 7.4 & 7.3 & 21.1 \\
\hline Median RPD for SRM = & 3.8 & & & & & & & & & & & & & \\
\hline GSD-8 laboratory & 3201 and 3202 & ICP/MS & 40600 & 1460 & 14400 & 21700 & 1770 & 3590 & 138 & 3970 & 2.80 & 482 & 2.26 & 0.063 \\
\hline GSD-8 literature & 3201 and 3202 & ICP/MS & 40800 & 1790 & 15380 & 23500 & 1510 & 3490 & 130 & 3660 & 2.4 & 480 & 2.00 & 0.08 \\
\hline RPD for each element $=$ & & & 0.5 & 20.3 & 6.6 & 8.0 & 15.9 & 2.8 & 6.0 & 8.1 & 15.4 & 0.4 & 12.2 & 25.0 \\
\hline Median RPD for SRM = & 8.1 & & & & & & & & & & & & & \\
\hline MAG-1 laboratory & 3209 & ICP/MS & 84700 & 10200 & 50600 & 30900 & 18700 & 28300 & 735 & 4450 & 9.67 & 495 & 3.04 & 0.366 \\
\hline MAG-1 literature & 3209 & ICP/MS & 86660 & 9790 & 47600 & 29500 & 18090 & 28400 & 711 & 4500 & 9.2 & 479 & 3.20 & 0.20 \\
\hline RPD for each element $=$ & & & 2.3 & 4.1 & 6.1 & 4.6 & 3.3 & 0.4 & 3.3 & 1.1 & 5.0 & 3.3 & 5.1 & 57.7 \\
\hline Median RPD for SRM = & 4.1 & & & & & & & & & & & & & \\
\hline NIST 2704 laboratory & 3209 & ICP/MS & 60700 & 25800 & 41900 & 20100 & 12600 & 5640 & 992 & 3170 & 22.0 & 418 & 1.91 & 3.49 \\
\hline NIST 2704 literature & 3209 & ICP/MS & 61090 & 26000 & 41100 & 20000 & 12000 & 5470 & 1000 & 4580 & 23.4 & 414 & & 3.45 \\
\hline RPD for each element $=$ & & & 0.6 & 0.8 & 1.9 & 0.5 & 4.9 & 3.1 & 0.8 & 36.4 & 6.2 & 1.0 & & 1.2 \\
\hline Median RPD for SRM = & 3.0 & & & & & & & & & & & & & \\
\hline SCo-1 laboratory & 3209 & ICP/MS & 74000 & 19000 & 37700 & 23000 & 17100 & 6730 & 952 & 3840 & 13.0 & 598 & 1.86 & 0.177 \\
\hline SCo-1 literature & 3209 & ICP/MS & 72370 & 18700 & 35900 & 23000 & 16400 & 6670 & 899 & 3760 & 12.4 & 570 & 1.84 & 0.14 \\
\hline RPD for each element $=$ & & & 2.2 & 1.6 & 4.9 & 0.0 & 4.2 & 0.9 & 5.7 & 2.1 & 4.7 & 4.8 & 1.1 & 23.3 \\
\hline Median RPD for SRM = & 4.7 & & & & & & & & & & & & & \\
\hline GSD-8 laboratory & 3209 & ICP/MS & 41600 & 1720 & 14000 & 24000 & 1590 & 3200 & 130 & 3820 & 2.93 & 456 & 1.81 & 0.048 \\
\hline GSD-8 literature & 3209 & ICP/MS & 40800 & 1790 & 15380 & 23500 & 1510 & 3490 & 130 & 3660 & 2.4 & 480 & 2.00 & 0.08 \\
\hline RPD for each element $=$ & & & 1.9 & 4.0 & 9.4 & 2.1 & 5.2 & 8.7 & 0.0 & 4.3 & 19.9 & 5.1 & 10.0 & 51.2 \\
\hline Median RPD for SRM = & 6.9 & & & & & & & & & & & & & \\
\hline MAG-1 laboratory & 3210 & ICP/MS & 85700 & 9910 & 51000 & 30200 & 19000 & 28200 & 759 & 4520 & 9.85 & 490 & 3.29 & 0.330 \\
\hline
\end{tabular}




\begin{tabular}{|c|c|c|c|c|c|c|c|c|c|c|c|c|c|c|}
\hline Sample ID & Job no. or Core & Method & $\mathrm{Al}, \mathrm{ppm}$ & $\mathrm{Ca}, \mathrm{ppm}$ & $\mathrm{Fe}, \mathrm{ppm}$ & $\mathrm{K}, \mathrm{ppm}$ & $\mathrm{Mg}, \mathrm{ppm}$ & $\mathrm{Na}, \mathrm{ppm}$ & $\mathrm{P}, \mathrm{ppm}$ & $\mathrm{Ti}, \mathrm{ppm}$ & As, ppm & Ba, ppm & Be, ppm & Cd, ppm \\
\hline MAG-1 literature & 3210 & ICP/MS & 866660 & 99790 & 447600 & 29500 & 18090 & 28400 & 711 & 4500 & 9.2 & 479 & 3.20 & 0.20 \\
\hline RPD for each element $=$ & & & 1.1 & 1.2 & 6.9 & 2.3 & 4.9 & 0.7 & 6.5 & 0.4 & 6.8 & 2.3 & 2.8 & 48.1 \\
\hline Median RPD for SRM = & 3.0 & & & & & & & & & & & & & \\
\hline NIST 2704 laboratory & 3210 & ICP/MS & 62800 & 25500 & 43000 & 19700 & 13100 & 5800 & 1050 & 3300 & 22.7 & 424 & 2.00 & 3.57 \\
\hline NIST 2704 literature & 3210 & ICP/MS & 61090 & 26000 & 41100 & 20000 & 12000 & 5470 & 1000 & 4580 & 23.4 & 414 & & 3.45 \\
\hline RPD for each element $=$ & & & 2.8 & 1.9 & 4.5 & 1.5 & 8.8 & 5.9 & 4.9 & 32.5 & 3.0 & 2.4 & & 3.4 \\
\hline Median RPD for SRM = & 3.3 & & & & & & & & & & & & & \\
\hline SCo-1 laboratory & 3210 & ICP/MS & 75400 & 18400 & 38600 & 22500 & 18100 & 7060 & 1010 & 4010 & 13.7 & 598 & 2.03 & 0.182 \\
\hline SCo-1 literature & 3210 & ICP/MS & 72370 & 18700 & 35900 & 23000 & 16400 & 6670 & 899 & 3760 & 12.4 & 570 & 1.84 & 0.14 \\
\hline RPD for each element $=$ & & & 4.1 & 1.6 & 7.2 & 2.2 & 9.9 & 5.7 & 11.6 & 6.4 & 10.0 & 4.8 & 9.8 & 26.1 \\
\hline Median RPD for SRM = & 6.5 & & & & & & & & & & & & & \\
\hline GSD-8 laboratory & 3210 & ICP/MS & 43400 & 1630 & 14200 & 23600 & 1690 & 3500 & 147 & 4100 & 3.07 & 468 & 2.01 & 0.069 \\
\hline GSD-8 literature & 3210 & ICP/MS & 40800 & 1790 & 15380 & 23500 & 1510 & 3490 & 130 & 3660 & 2.4 & 480 & 2.00 & 0.08 \\
\hline RPD for each element $=$ & & & 6.2 & 9.4 & 8.0 & 0.4 & 11.3 & 0.3 & 12.3 & 11.3 & 24.5 & 2.5 & 0.5 & 16.0 \\
\hline Median RPD for SRM = & 8.0 & & & & & & & & & & & & & \\
\hline MAG-1 laboratory & 3342 and 3427 & ICP/MS & 91000 & 10400 & 51100 & 31900 & 19000 & 29600 & 786 & 4900 & 10.4 & 487 & 3.21 & 0.225 \\
\hline MAG-1 literature & 3342 and 3427 & ICP/MS & 86660 & 9790 & 47600 & 29500 & 18090 & 28400 & 711 & 4500 & 9.2 & 479 & 3.20 & 0.20 \\
\hline $\begin{array}{r}\text { RPD for each element }= \\
\text { Median RPD for SRM }=\end{array}$ & 7.1 & & 4.9 & 6.0 & 7.1 & 7.8 & 4.9 & 4.1 & 10.0 & 8.5 & 12.2 & 1.7 & 0.3 & 10.8 \\
\hline NIST 2704 laboratory & 3342 and 3427 & ICP/MS & 63900 & 26800 & 42200 & 20500 & 12200 & 5710 & 1060 & 3250 & 22.3 & 402 & 1.90 & 3.50 \\
\hline NIST 2704 literature & 3342 and 3427 & ICP/MS & 61090 & 26000 & 41100 & 20000 & 12000 & 5470 & 1000 & 4580 & 23.4 & 414 & & 3.45 \\
\hline RPD for each element $=$ & & & 4.5 & 3.0 & 2.6 & 2.5 & 1.7 & 4.3 & 5.8 & 34.0 & 4.8 & 2.9 & & 1.4 \\
\hline Median RPD for SRM = & 4.1 & & & & & & & & & & & & & \\
\hline ScO-1 laboratory & 3342 and 3427 & ICP/MS & 76300 & 19200 & 37800 & 23900 & 16400 & 6580 & 991 & 4060 & 13.0 & 570 & 1.79 & 0.149 \\
\hline SCO-1 literature & 3342 and 3427 & ICP/MS & 72370 & 18700 & 35900 & 23000 & 16400 & 6670 & 899 & 3760 & 12.4 & 570 & 1.84 & 0.14 \\
\hline RPD for each element $=$ & & & 5.3 & 2.6 & 5.2 & 3.8 & 0.0 & 1.4 & 9.7 & 7.7 & 4.7 & 0.0 & 2.8 & 6.2 \\
\hline Median RPD for SRM = & 4.7 & & & & & & & & & & & & & \\
\hline GSD-8 laboratory & 3342 and 3427 & ICP/MS & 43400 & 1550 & 15600 & 25000 & 1360 & 2970 & 145 & 4070 & 2.88 & 414 & 1.73 & 0.194 \\
\hline GSD-8 literature & 3342 and 3427 & ICP/MS & 40800 & 1790 & 15380 & 23500 & 1510 & 3490 & 130 & 3660 & 2.4 & 480 & 2.00 & 0.08 \\
\hline RPD for each element $=$ & & & 6.2 & 14.4 & 1.4 & 6.2 & 10.5 & 16.1 & 10.9 & 10.6 & 18.2 & 14.8 & 14.5 & 82.2 \\
\hline Median RPD for SRM = & 10.5 & & & & & & & & & & & & & \\
\hline MAG-1 laboratory & 3711 & ICP/MS & 88200 & 10000 & 49400 & 31000 & 19400 & 30000 & 766 & 4090 & 10.4 & 517 & 3.28 & 0.228 \\
\hline MAG-1 literature & 3711 & ICP/MS & 86660 & 9790 & 47600 & 29500 & 18090 & 28400 & 711 & 4500 & 9.2 & 479 & 3.20 & 0.20 \\
\hline RPD for each element $=$ & & & 1.8 & 2.1 & 3.7 & 5.0 & 7.0 & 5.5 & 7.4 & 9.5 & 12.2 & 7.6 & 2.5 & 12.1 \\
\hline Median RPD for SRM = & 6.0 & & & & & & & & & & & & & \\
\hline NIST 2704 laboratory & 3711 & ICP/MS & 61700 & 26400 & 40700 & 20000 & 12200 & 5650 & 1020 & 3230 & 22.8 & 419 & 1.90 & 3.56 \\
\hline NIST 2704 literature & 3711 & ICP/MS & 61090 & 26000 & 41100 & 20000 & 12000 & 5470 & 1000 & 4580 & 23.4 & 414 & & 3.45 \\
\hline RPD for each element $=$ & & & 1.0 & 1.5 & 1.0 & 0.0 & 1.7 & 3.2 & 2.0 & 34.6 & 2.6 & 1.2 & & 3.1 \\
\hline Median RPD for SRM = & 2.4 & & & & & & & & & & & & & \\
\hline SCo-1 laboratory & 3711 & ICP/MS & 73200 & 18400 & 36300 & 23100 & 15900 & 6380 & 926 & 3560 & 12.9 & 589 & 1.96 & 0.156 \\
\hline SCo-1 literature & 3711 & ICP/MS & 72370 & 18700 & 35900 & 23000 & 16400 & 6670 & 899 & 3760 & 12.4 & 570 & 1.84 & 0.14 \\
\hline RPD for each element $=$ & & & 1.1 & 1.6 & 1.1 & 0.4 & 3.1 & 4.4 & 3.0 & 5.5 & 4.0 & 3.3 & 6.3 & 10.8 \\
\hline Median RPD for SRM = & 4.0 & & & & & & & & & & & & & \\
\hline GSD-8 laboratory & 3711 & ICP/MS & 40200 & 1470 & 15100 & 24000 & 1470 & 3050 & 129 & 3620 & 2.89 & 447 & 2.12 & 0.043 \\
\hline GSD-8 literature & 3711 & ICP/MS & 40800 & 1790 & 15380 & 23500 & 1510 & 3490 & 130 & 3660 & 2.4 & 480 & 2.00 & 0.08 \\
\hline RPD for each element $=$ & & & 1.5 & 19.6 & 1.8 & 2.1 & 2.7 & 13.5 & 0.8 & 1.1 & 18.5 & 7.1 & 5.8 & 61.3 \\
\hline Median RPD for SRM = & 5.8 & & & & & & & & & & & & & \\
\hline
\end{tabular}




\begin{tabular}{|c|c|c|c|c|c|c|c|c|c|c|c|c|c|c|}
\hline Sample ID & Job no. or Core & Method & $\mathrm{Al}, \mathrm{ppm}$ & $\mathrm{Ca}, \mathrm{ppm}$ & $\mathrm{Fe}, \mathrm{ppm}$ & $\mathrm{K}, \mathrm{ppm}$ & $\mathrm{Mg}, \mathrm{ppm}$ & $\mathrm{Na}, \mathrm{ppm}$ & $\mathrm{P}, \mathrm{ppm}$ & Ti, ppm & As, ppm & Ba, ppm & $\mathrm{Be}, \mathrm{ppm}$ & $\mathrm{Cd}, \mathrm{ppm}$ \\
\hline MAG-1 laboratory & (3744 & $\overline{I I C P / M S}$ & 899975 & $=10350$ & 499725 & 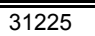 & (19825 & 30225 & $7 \overline{757}$ & $=4523$ & 9.7 & 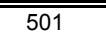 & 3.4 .4 & 0.25 \\
\hline MAG-1 literature & 3744 & ICP/MS & 86660 & 9790 & 47600 & 29500 & 18090 & 28400 & 711 & 4500 & 9.2 & 479 & 3.20 & 0.20 \\
\hline RPD for each element $=$ & & & 3.8 & 5.6 & 4.4 & 5.7 & 9.2 & 6.2 & 6.3 & 0.5 & 5.7 & 4.5 & 4.7 & 20.1 \\
\hline Median RPD for SRM = & 5.6 & & & & & & & & & & & & & \\
\hline NIST 2704 laboratory & 3744 & ICP/MS & 61950 & 26350 & 41000 & 20100 & 13300 & 6060 & 1010 & 3090 & 22.0 & 429 & 2.0 & 3.6 \\
\hline NIST 2704 literature & 3744 & ICP/MS & 61090 & 26000 & 41100 & 20000 & 12000 & 5470 & 1000 & 4580 & 23.4 & 414 & & 3.45 \\
\hline RPD for each element $=$ & & & 1.4 & 1.3 & 0.2 & 0.5 & 10.3 & 10.2 & 1.0 & 38.9 & 6.4 & 3.4 & & 4.1 \\
\hline Median RPD for SRM = & 3.1 & & & & & & & & & & & & & \\
\hline SCO-1 laboratory & 3744 & ICP/MS & 73800 & 18700 & 36750 & 23000 & 17800 & 7065 & 951 & 3790 & 12.8 & 602 & 2.1 & 0.16 \\
\hline SCO-1 literature & 3744 & ICP/MS & 72370 & 18700 & 35900 & 23000 & 16400 & 6670 & 899 & 3760 & 12.4 & 570 & 1.84 & 0.14 \\
\hline RPD for each element $=$ & & & 2.0 & 0.0 & 2.3 & 0.0 & 8.2 & 5.8 & 5.6 & 0.8 & 3.2 & 5.4 & 13.9 & 12.1 \\
\hline Median RPD for SRM = & 5.6 & & & & & & & & & & & & & \\
\hline GSD-8 laboratory & 3744 & ICP/MS & 40750 & 1545 & 15600 & 24100 & 1525 & 3320 & 129 & 4145 & 2.7 & 459 & 2.2 & 0.05 \\
\hline GSD-8 literature & 3744 & ICP/MS & 40800 & 1790 & 15380 & 23500 & 1510 & 3490 & 130 & 3660 & 2.40 & 480 & 2.00 & 0.08 \\
\hline RPD for each element $=$ & & & 0.1 & 14.7 & 1.4 & 2.5 & 1.0 & 5.0 & 0.8 & 12.4 & 12.5 & 4.5 & 9.8 & 39.1 \\
\hline Median RPD for SRM = & 8.8 & & & & & & & & & & & & & \\
\hline MAG-1 laboratory & 3784 & ICP/MS & 91400 & 10500 & 51400 & 31700 & 19700 & 29900 & 759 & 4900 & 9.59 & 504 & 3.17 & 0.275 \\
\hline MAG-1 literature & 3784 & ICP/MS & 86660 & 9790 & 47600 & 29500 & 18090 & 28400 & 711 & 4500 & 9.2 & 479 & 3.20 & 0.20 \\
\hline RPD for each element $=$ & & & 5.3 & 7.0 & 7.7 & 7.2 & 8.5 & 5.1 & 6.5 & 8.5 & 4.2 & 5.1 & 0.9 & 30.6 \\
\hline Median RPD for SRM $=$ & 6.5 & & & & & & & & & & & & & \\
\hline NIST 2704 laboratory & 3784 & ICP/MS & 63500 & 26100 & 42200 & 20000 & 12800 & 5880 & 1010 & 2880 & 21.7 & 426 & 1.94 & 3.54 \\
\hline NIST 2704 literature & 3784 & ICP/MS & 61090 & 26000 & 41100 & 20000 & 12000 & 5470 & 1000 & 4580 & 23.4 & 414 & & 3.45 \\
\hline RPD for each element $=$ & & & 3.9 & 0.4 & 2.6 & 0.0 & 6.5 & 7.2 & 1.0 & 45.6 & 7.5 & 2.9 & & 2.6 \\
\hline Median RPD for SRM = & 3.0 & & & & & & & & & & & & & \\
\hline SCo-1 laboratory & 3784 & ICP/MS & 79900 & 19300 & 39400 & 24000 & 18200 & 7080 & 978 & 3940 & 13.0 & 610 & 1.84 & 0.148 \\
\hline SCo-1 literature & 3784 & ICP/MS & 72370 & 18700 & 35900 & 23000 & 16400 & 6670 & 899 & 3760 & 12.4 & 570 & 1.84 & 0.14 \\
\hline RPD for each element $=$ & & & 9.9 & 3.2 & 9.3 & 4.3 & 10.4 & 6.0 & 8.4 & 4.7 & 4.7 & 6.8 & 0.0 & 5.6 \\
\hline Median RPD for SRM = & 6.0 & & & & & & & & & & & & & \\
\hline GSD-8 laboratory & 3784 & ICP/MS & 46500 & 1620 & 16800 & 25600 & 1620 & 3470 & 136 & 4670 & 2.66 & 470 & 1.91 & 0.047 \\
\hline GSD-8 literature & 3784 & ICP/MS & 40800 & 1790 & 15380 & 23500 & 1510 & 3490 & 130 & 3660 & 2.4 & 480 & 2.00 & 0.08 \\
\hline RPD for each element $=$ & & & 13.1 & 10.0 & 8.8 & 8.6 & 7.0 & 0.6 & 4.5 & 24.2 & 10.3 & 2.1 & 4.6 & 53.1 \\
\hline Median RPD for SRM = & 8.8 & & & & & & & & & & & & & \\
\hline MAG-1 laboratory & 3818 & ICP/MS & 90500 & 10400 & 50500 & 31400 & 18600 & 28000 & 757 & 4370 & 10.6 & 506 & 3.48 & 0.250 \\
\hline MAG-1 literature & 3818 & ICP/MS & 86660 & 9790 & 47600 & 29500 & 18090 & 28400 & 711 & 4500 & 9.2 & 479 & 3.20 & 0.20 \\
\hline RPD for each element $=$ & & & 4.3 & 6.0 & 5.9 & 6.2 & 2.8 & 1.4 & 6.3 & 2.9 & 14.1 & 5.5 & 8.4 & 21.2 \\
\hline Median RPD for SRM = & 6.0 & & & & & & & & & & & & & \\
\hline NIST 2704 laboratory & 3818 & ICP/MS & 63100 & 26900 & 41800 & 20600 & 12600 & 5510 & 1000 & 3200 & 22.5 & 421 & 2.27 & 3.71 \\
\hline NIST 2704 literature & 3818 & ICP/MS & 61090 & 26000 & 41100 & 20000 & 12000 & 5470 & 1000 & 4580 & 23.4 & 414 & & 3.45 \\
\hline RPD for each element $=$ & & & 3.2 & 3.4 & 1.7 & 3.0 & 4.9 & 0.7 & 0.0 & 35.5 & 3.9 & 1.7 & & 7.3 \\
\hline Median RPD for SRM = & 3.3 & & & & & & & & & & & & & \\
\hline SCo-1 laboratory & 3818 & ICP/MS & 75700 & 19400 & 38400 & 23600 & 17300 & 6660 & 963 & 3790 & 13.0 & 593 & 2.15 & 0.162 \\
\hline SCo-1 literature & 3818 & ICP/MS & 72370 & 18700 & 35900 & 23000 & 16400 & 6670 & 899 & 3760 & 12.4 & 570 & 1.84 & 0.14 \\
\hline RPD for each element $=$ & & & 4.5 & 3.7 & 6.7 & 2.6 & 5.3 & 0.2 & 6.9 & 0.8 & 4.7 & 4.0 & 15.5 & 14.6 \\
\hline Median RPD for SRM = & 4.7 & & & & & & & & & & & & & \\
\hline GSD-8 laboratory & 3818 & ICP/MS & 41600 & 1560 & 15800 & 24500 & 1620 & 3290 & 127 & 3830 & 2.79 & 446 & 2.36 & 0.101 \\
\hline GSD-8 literature & 3818 & ICP/MS & 40800 & 1790 & 15380 & 23500 & 1510 & 3490 & 130 & 3660 & 2.4 & 480 & 2.00 & 0.08 \\
\hline RPD for each element $=$ & & & 1.9 & 13.7 & 2.7 & 4.2 & 7.0 & 5.9 & 2.3 & 4.5 & 15.0 & 7.3 & 16.5 & 22.0 \\
\hline Median RPD for SRM = & 5.9 & & & & & & & & & & & & & \\
\hline
\end{tabular}


Sample ID

Job no. or Core Method

$\mathrm{Al}, \mathrm{ppm} \quad \mathrm{Ca}, \mathrm{ppm}$

Fe, ppm

\begin{tabular}{llll}
$\mathrm{K}, \mathrm{ppm} \quad \mathrm{Mg}, \mathrm{ppm} \quad \mathrm{Na}, \mathrm{ppm} \quad \mathrm{P}, \mathrm{ppm}$ \\
\hline
\end{tabular}

$\begin{array}{lllll}\text { Ti, ppm } & \text { As, ppm } & \text { Ba, ppm } & \text { Be, ppm } & \text { Cd, ppm }\end{array}$

MAG-1 laboratory

ICP/MS

$\begin{array}{ll}- & 10000\end{array}$

$10000 \quad 50100$

$50100 \quad 308$

$30800 \quad 18400$

$18400 \quad 28800$

28800

756

$4410 \quad 9.38$

$\mathrm{RPD}$ for each element $=$ Median RPD for SRM =

NIST 2704 laboratory
NIST 2704 literature

3.9

$2.1 \quad 5.1$

3.9
4315

47600
5.1

4.3

1.7

1.4

711
6.1

4500
2.0

$2.0 \quad 1.9$

$\begin{array}{lll}496 & 3.36 & 0.254 \\ 479 & 3.20 & 0.20\end{array}$

RPD for each element

ICP/MS

$62400 \quad 25600$

41400

$\begin{array}{llll}19800 & 12200 & 5700 & 1010\end{array}$

$\begin{array}{lllll}3110 & 21.1 & 416 & 2.02 & 3.57\end{array}$

Median RPD for SRM =

SCO-1 laboratory

SCO-1 literature
RPD for each element $=$

Median RPD for SRM =

GSD-8 laboratory

4315

21

1.6

0.7

$20000 \quad 12000$

5470

$\begin{array}{lll}1000 & 4580 & 21.1 \\ 1.0 & 38.2 & 10.3\end{array}$

414

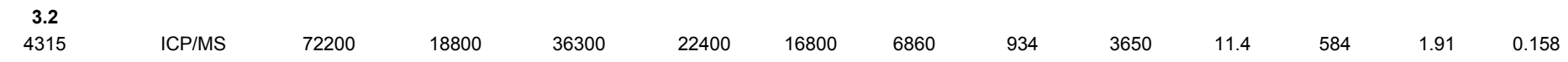

4315

ICP/MS

$\begin{array}{ccc}72370 & 18700 \quad 35900 \\ 0.2 & 0.5 & 1.1\end{array}$

$6860 \quad 934$

3650

$\begin{array}{llll}11.4 & 584 & 1.91 & 0.158 \\ 12.4 & 570 & 1.84 & 0.14\end{array}$

3.0

ICP/MS

0.5

1.1

$2.6 \quad 2$

2.8

3.8

3760
3.0

8.4

$\begin{array}{lll}\mathbf{2 . 4} & \mathbf{3 . 7} & \mathbf{1 2 . 1}\end{array}$

GSD-8 literature

$4315 \quad$ ICP/MS

$40800 \quad 1790$

15700

$23800 \quad 1470$

$\begin{array}{ll}3110 & 129 \\ 3490 & 130\end{array}$

$\begin{array}{lllll}3760 & 1.18 & 456 & 2.04 & 0.043 \\ 3660 & 2.4 & 480 & 2.00 & 0.08\end{array}$

$1.0 \quad 22.4$

2.1

1.3

$\begin{array}{cc}1510 & 3490 \\ 2.7 & 11.5\end{array}$

0.8

5.1

$2.00 \quad 0.08$

\section{ICP/MS METHOD}

Median RPD for MAG-1 for each element:

Median RPD for NIST 2704 for each element:

Median RPD for SCo-1 for each element:

Median RPD for GSD-8 for each element:

$\begin{array}{lll}3.8 & 3.1 & 5.5 \\ 2.4 & 1.6 & 1.3 \\ 3.2 & 2.1 & 4.2 \\ 1.9 & 14.1 & 4.6\end{array}$

$\begin{array}{ll}5.5 & 4.8 \\ 1.3 & 0.8 \\ 4.2 & 2.6 \\ 4.6 & 3.3\end{array}$

$\begin{array}{ll}4.8 & 4.1 \\ 0.8 & 4.9 \\ 2.6 & 4.8 \\ 3.3 & 6.1\end{array}$

$\begin{array}{ll}4.1 & 1.4 \\ 4.9 & 4.2 \\ 4.8 & 2.7 \\ 6.1 & 7.3\end{array}$

$\begin{array}{ll}1.4 & 6.3 \\ 4.2 & 1.0 \\ 2.7 & 5.6 \\ 7.3 & 2.7\end{array}$

$\begin{array}{ll}6.3 & 3.9 \\ 1.0 & 37.3 \\ 5.6 & 4.2 \\ 2.7 & 7.7\end{array}$

$\begin{array}{cc}3.9 & 6.1 \\ 37.3 & 5.0 \\ 4.2 & 4.7 \\ 7.7 & 16.8\end{array}$

$\begin{array}{cccc}6.1 & 4.8 & 3.5 & 26.7 \\ 5.0 & 2.6 & & 3.3 \\ 4.7 & 4.4 & 5.0 & 12.1 \\ 16.8 & 4.9 & 7.8 & 52.1\end{array}$




\begin{tabular}{|c|c|c|c|c|c|c|c|c|c|c|c|c|}
\hline Sample ID & Co, ppm & $\mathrm{Cr}, \mathrm{ppm}$ & $\mathrm{Cu}, \mathrm{ppm}$ & $\mathrm{Hg}, \mathrm{ppm}$ & $\mathrm{Li}, \mathrm{ppm}$ & $\mathrm{Mn}, \mathrm{ppm}$ & $\mathrm{Ni}, \mathrm{ppm}$ & $\mathrm{Pb}, \mathrm{ppm}$ & Sc, ppm & $\mathrm{Sr}, \mathrm{ppm}$ & V, ppm & $\mathrm{Zn}, \mathrm{ppm}$ \\
\hline MAG-1 laboratory & 23.05 & 999.00 & 230.85 & $\begin{array}{l}<.020 \\
\end{array}$ & 7979.65 & (769.00 & $=53.70$ & 2727.00 & 1919.40 & $1+144.00$ & 13139.00 & 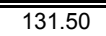 \\
\hline MAG-1 literature & 20.4 & 97 & 30.0 & $.0179 ?$ & 79.0 & 760 & 53.0 & 24.0 & 17.2 & 146 & 140 & 130 \\
\hline $\begin{array}{r}\text { RPD for each element }= \\
\text { Median RPD for SRM }=\end{array}$ & 12.2 & 2.0 & 2.8 & & 0.8 & 1.2 & 1.3 & 11.8 & 12.0 & 1.4 & 0.7 & 1.1 \\
\hline NIST 2704 laboratory & 14.23 & 133.67 & 98.63 & 1.49 & 47.77 & 561.33 & 43.53 & 163.00 & 12.03 & 131.33 & 93.33 & 440.33 \\
\hline NIST 2704 literature & 14.0 & 135 & 98.6 & 1.44 & 47.5 & 555 & 44.1 & 161.0 & 12.0 & 130 & 95.0 & 438 \\
\hline $\begin{array}{r}\text { RPD for each element }= \\
\text { Median RPD for SRM }=\end{array}$ & 1.7 & 1.0 & 0.0 & 3.2 & 0.6 & 1.1 & 1.3 & 1.2 & 0.3 & 1.0 & 1.8 & 0.5 \\
\hline SCo-1 laboratory & 11.50 & 67.00 & 29.05 & 0.06 & 45.15 & 413.00 & 27.15 & 29.50 & 11.70 & 177.00 & 128.00 & 103.00 \\
\hline SCo-1 literature & 10.5 & 68.0 & 28.7 & 0.062 & 45.0 & 410 & 27.0 & 31.0 & 10.8 & 174 & 131 & 103 \\
\hline $\begin{array}{r}\text { RPD for each element }= \\
\text { Median RPD for SRM }=\end{array}$ & 9.1 & 1.5 & 1.2 & 0.8 & 0.3 & 0.7 & 0.6 & 5.0 & 8.0 & 1.7 & 2.3 & 0.0 \\
\hline GSD-8 laboratory & 3.75 & 7.60 & 4.25 & 0.05 & 13.15 & 308.00 & 2.60 & 19.50 & 6.00 & 52.00 & 25.50 & 44.00 \\
\hline GSD-8 literature & 3.6 & 7.60 & 4.1 & 0.042 & 13.2 & 310 & 2.7 & 21.0 & 5.7 & 52.0 & 26 & 43.0 \\
\hline $\begin{array}{r}\text { RPD for each element }= \\
\text { Median RPD for SRM }=\end{array}$ & 4.1 & 0.0 & 3.6 & 6.9 & 0.4 & 0.6 & 3.8 & 7.4 & 5.1 & 0.0 & 1.9 & 2.3 \\
\hline MESS-2 laboratory & 14.20 & 103.00 & 40.15 & 0.09 & 73.65 & 370.00 & 48.85 & 22.50 & 9.75 & 127.00 & 247.00 & 172.00 \\
\hline MESS-2 literature (no symbols) & 13.8 & 106 & 39.3 & 0.092 & 73.9 & 365 & 49.3 & 21.9 & & 125 & 252 & 172 \\
\hline MESS-2 literature & $13.8 \pm 1.4$ & $106 \pm 8$ & $39.3 \pm 2.0$ & $0.092 \pm 0.009$ & $73.9 \pm 0.7$ & $365 \pm 21$ & $49.3 \pm 1.8$ & $21.9 \pm 1.2$ & & $125 \pm 10$ & $252 \pm 10$ & $172 \pm 16$ \\
\hline $\begin{array}{r}\text { RPD for each element }= \\
\text { Median RPD for SRM }=\end{array}$ & 2.9 & 2.9 & 2.1 & 1.6 & 0.3 & 1.4 & 0.9 & 2.7 & & 1.6 & 2.0 & 0.0 \\
\hline MAG-1 laboratory & 20.3 & 97 & 29.9 & $<.020$ & 79.4 & 772 & 54 & 25 & 18.7 & 142 & 140 & 132 \\
\hline MAG-1 literature & 20.4 & 97 & 30.0 & $.0179 ?$ & 79.0 & 760 & 53.0 & 24.0 & 17.2 & 146 & 140 & 130 \\
\hline $\begin{array}{r}\text { RPD for each element }= \\
\text { Median RPD for SRM }=\end{array}$ & 0.5 & 0.0 & 0.3 & & 0.5 & 1.6 & 1.9 & 4.1 & 8.4 & 2.8 & 0.0 & 1.5 \\
\hline NIST 2704 laboratory & 17.1 & 131 & 96.7 & 1.4 & 49.4 & 572 & 43.9 & 155 & 12 & 125 & 97 & 453 \\
\hline NIST 2704 literature & 14.0 & 135 & 98.6 & 1.44 & 47.5 & 555 & 44.1 & 161.0 & 12.0 & 130 & 95.0 & 438 \\
\hline $\begin{array}{r}\text { RPD for each element }= \\
\text { Median RPD for SRM }=\end{array}$ & 19.9 & 3.0 & 1.9 & 2.8 & 3.9 & 3.0 & 0.5 & 3.8 & 0.0 & 3.9 & 2.1 & 3.4 \\
\hline SCo-1 laboratory & 12.0 & 68 & 27.5 & 0.07 & 45.4 & 411 & 28 & 32 & 11 & 169 & 134 & 103 \\
\hline SCo-1 literature & 10.5 & 68.0 & 28.7 & 0.062 & 45.0 & 410 & 27.0 & 31.0 & 10.8 & 174 & 131 & 103 \\
\hline $\begin{array}{r}\text { RPD for each element }= \\
\text { Median RPD for SRM }=\end{array}$ & 13.3 & 0.0 & 4.3 & 12.1 & 0.9 & 0.2 & 3.6 & 3.2 & 1.8 & 2.9 & 2.3 & 0.0 \\
\hline GSD-8 laboratory & 3.2 & 7.8 & 4 & 0.039 & 13.0 & 313 & 2.4 & 23 & 5.8 & 51 & 25 & 44 \\
\hline GSD-8 literature & 3.6 & 7.60 & 4.1 & 0.042 & 13.2 & 310 & 2.7 & 21.0 & 5.7 & 52.0 & 26 & 43.0 \\
\hline $\begin{array}{r}\text { RPD for each element }= \\
\text { Median RPD for SRM }=\end{array}$ & 11.8 & 2.6 & 2.5 & 7.4 & 1.5 & 1.0 & 11.8 & 9.1 & 1.7 & 1.9 & 3.9 & 2.3 \\
\hline MESS-2 laboratory & 13.6 & 104 & 39.2 & 0.099 & 74.6 & 364 & 48.9 & 22 & 9.6 & 124 & 252 & 176 \\
\hline MESS-2 literature (no symbols) & 13.8 & 106 & 39.3 & 0.092 & 73.9 & 365 & 49.3 & 21.9 & & 125 & 252 & 172 \\
\hline MESS-2 literature & $13.8 \pm 1.4$ & $106 \pm 8$ & $39.3 \pm 2.0$ & $0.092 \pm 0.009$ & $73.9 \pm 0.7$ & $365 \pm 21$ & $49.3 \pm 1.8$ & $21.9 \pm 1.2$ & & $125 \pm 10$ & $252 \pm 10$ & $172 \pm 16$ \\
\hline $\begin{array}{r}\text { RPD for each element }= \\
\text { Median RPD for SRM }=\end{array}$ & 1.5 & 1.9 & 0.3 & 7.3 & 0.9 & 0.3 & 0.8 & 0.5 & & 0.8 & 0.0 & 2.3 \\
\hline MAG-1 laboratory & 20.0 & 97 & 30.0 & $<.020$ & 79.3 & 768 & 54.1 & 26 & 16.5 & 150 & 140 & 127 \\
\hline MAG-1 literature & 20.4 & 97 & 30.0 & $.0179 ?$ & 79.0 & 760 & 53.0 & 24.0 & 17.2 & 146 & 140 & 130 \\
\hline RPD for each element $=$ & 2.0 & 0.0 & 0.0 & & 0.4 & 1.0 & 2.1 & 8.0 & 4.2 & 2.7 & 0.0 & 2.3 \\
\hline
\end{tabular}

Median RPD for SRM = 


\begin{tabular}{|c|c|c|c|c|c|c|c|c|c|c|c|c|}
\hline Sample ID & Co, ppm & $\mathrm{Cr}, \mathrm{ppm}$ & $\mathrm{Cu}, \mathrm{ppm}$ & $\mathrm{Hg}, \mathrm{ppm}$ & $\mathrm{Li}, \mathrm{ppm}$ & $\mathrm{Mn}, \mathrm{ppm}$ & $\mathrm{Ni}, \mathrm{ppm}$ & $\mathrm{Pb}, \mathrm{ppm}$ & Sc, ppm & Sr, ppm & $\mathrm{V}, \mathrm{ppm}$ & $\mathrm{Zn}, \mathrm{ppm}$ \\
\hline NIST 2704 laboratory & 13.1 & 140 & 96.8 & 1.47 & 48.1 & 550 & 44.9 & 158 & 11.5 & 128 & 95 & 447 \\
\hline NIST 2704 literature & 14.0 & 135 & 98.6 & 1.44 & 47.5 & 555 & 44.1 & 161.0 & 12.0 & 130 & 95.0 & 438 \\
\hline $\begin{array}{r}\text { RPD for each element }= \\
\text { Median RPD for SRM }=\end{array}$ & 6.6 & 3.6 & 1.8 & & 1.3 & 0.9 & 1.8 & 1.9 & 4.3 & 1.6 & 0.0 & 2.0 \\
\hline SCo-1 laboratory & 9.6 & 71 & 28.4 & 0.053 & 44.9 & 411 & 27.4 & 32 & 11.3 & 176 & 131 & 107 \\
\hline SCo-1 literature & 10.5 & 68.0 & 28.7 & $.062 ?$ & 45.0 & 410 & 27.0 & 31.0 & 10.8 & 174 & 131 & 103 \\
\hline $\begin{array}{r}\text { RPD for each element }= \\
\text { Median RPD for SRM }=\end{array}$ & 9.0 & 4.3 & 1.1 & & 0.2 & 0.2 & 1.5 & 3.2 & 4.5 & 1.1 & 0.0 & 3.8 \\
\hline GSD-8 laboratory & 4 & 7.4 & 4.2 & 0.043 & 13.5 & 306 & 3.1 & 24 & 5.8 & 52 & 26 & 44 \\
\hline GSD-8 literature & 3.6 & 7.60 & 4.1 & 0.042 & 13.2 & 310 & 2.7 & 21.0 & 5.7 & 52.0 & 26 & 43.0 \\
\hline $\begin{array}{r}\text { RPD for each element }= \\
\text { Median RPD for SRM }=\end{array}$ & 10.5 & 2.7 & 2.4 & & 2.2 & 1.3 & 13.8 & 13.3 & 1.7 & 0.0 & 0.0 & 2.3 \\
\hline MESS-2 laboratory & 14.3 & 107 & 39.3 & $\mathrm{n} / \mathrm{a}$ & 73.9 & 375 & 49.7 & 23 & 9.4 & 127 & 255 & 175 \\
\hline MESS-2 literature (no symbols) & 13.8 & 106 & 39.3 & 0.092 & 73.9 & 365 & 49.3 & 21.9 & & 125 & 252 & 172 \\
\hline MESS-2 literature & $13.8 \pm 1.4$ & $106 \pm 8$ & $39.3 \pm 2.0$ & $0.092 \pm 0.009$ & $73.9 \pm 0.7$ & $365 \pm 21$ & $49.3 \pm 1.8$ & $21.9 \pm 1.2$ & & $125 \pm 10$ & $252 \pm 10$ & $172 \pm 16$ \\
\hline $\begin{array}{r}\text { RPD for each element }= \\
\text { Median RPD for SRM }=\end{array}$ & 3.6 & 0.9 & 0.0 & & 0.0 & 2.7 & 0.8 & 4.9 & & 1.6 & 1.2 & 1.7 \\
\hline MAG-1 laboratory & 21.6 & 92 & 29.9 & $<.020$ & 126.8 & 758 & 54.3 & 33 & 17.7 & 158 & 139 & 133 \\
\hline MAG-1 literature & 20.4 & 97 & 30.0 & $.0179 ?$ & 79.0 & 760 & 53.0 & 24.0 & 17.2 & 146 & 140 & 130 \\
\hline $\begin{array}{r}\text { RPD for each element }= \\
\text { Median RPD for SRM }=\end{array}$ & 5.7 & 5.3 & 0.3 & & 46.5 & 0.3 & 2.4 & 31.6 & 2.9 & 7.9 & 0.7 & 2.3 \\
\hline NIST 2704 laboratory & 14.4 & 112 & 98.8 & 1.48 & 65.4 & 560 & 43.2 & 138 & 11.4 & 129 & 96 & 433 \\
\hline NIST 2704 literature & 14.0 & 135 & 98.6 & 1.44 & 47.5 & 555 & 44.1 & 161.0 & 12.0 & 130 & 95.0 & 438 \\
\hline $\begin{array}{r}\text { RPD for each element }= \\
\text { Median RPD for SRM }=\end{array}$ & 2.8 & 18.6 & 0.2 & 2.7 & 31.7 & 0.9 & 2.1 & 15.4 & 5.1 & 0.8 & 1.0 & 1.1 \\
\hline SCo-1 laboratory & 10.9 & 65 & 28.9 & $\mathrm{n} / \mathrm{a}$ & 67.2 & 403 & 28.5 & 35 & 11.7 & 170 & 132 & 103 \\
\hline SCo-1 literature & 10.5 & 68.0 & 28.7 & $.062 ?$ & 45.0 & 410 & 27.0 & 31.0 & 10.8 & 174 & 131 & 103 \\
\hline $\begin{array}{r}\text { RPD for each element }= \\
\text { Median RPD for SRM }=\end{array}$ & 3.7 & 4.5 & 0.7 & & 39.6 & 1.7 & 5.4 & 12.1 & 8.0 & 2.3 & 0.8 & 0.0 \\
\hline GSD-8 laboratory & 4 & 8.2 & 4 & 0.043 & 19.9 & 317 & 2.6 & 23 & 6 & 53 & 25 & 43 \\
\hline GSD-8 literature & 3.6 & 7.60 & 4.1 & 0.042 & 13.2 & 310 & 2.7 & 21.0 & 5.7 & 52.0 & 26 & 43.0 \\
\hline $\begin{array}{r}\text { RPD for each element }= \\
\text { Median RPD for SRM }=\end{array}$ & 10.5 & 7.6 & 2.5 & 2.4 & 40.5 & 2.2 & 3.8 & 9.1 & 5.1 & 1.9 & 3.9 & 0.0 \\
\hline MESS-2 laboratory & 14.9 & 112 & 40 & 0.09 & 75.5 & 355 & 49.4 & 23 & 9.6 & 130 & 255 & 170 \\
\hline MESS-2 literature (no symbols) & 13.8 & 106 & 39.3 & 0.092 & 73.9 & 365 & 49.3 & 21.9 & & 125 & 252 & 172 \\
\hline MESS-2 literature & $13.8 \pm 1.4$ & $106 \pm 8$ & $39.3 \pm 2.0$ & $0.092 \pm 0.009$ & $73.9 \pm 0.7$ & $365 \pm 21$ & $49.3 \pm 1.8$ & $21.9 \pm 1.2$ & & $125 \pm 10$ & $252 \pm 10$ & $172 \pm 16$ \\
\hline $\begin{array}{r}\text { RPD for each element }= \\
\text { Median RPD for SRM }=\end{array}$ & 7.7 & 5.5 & 1.8 & 2.2 & 2.1 & 2.8 & 0.2 & 4.9 & & 3.9 & 1.2 & 1.2 \\
\hline ICPIAES $\Lambda$ & & & & & & & & & & & & \\
\hline Median RPD for MAG-1 & 3.8 & 1.0 & 0.3 & & 0.7 & 1.1 & 2.0 & 9.9 & 6.3 & 2.7 & 0.4 & 1.9 \\
\hline Median RPD for NIST 2704 & 4.7 & 3.3 & 1.0 & 2.8 & 2.6 & 1.0 & 1.5 & 2.8 & 2.3 & 1.3 & 1.4 & 1.6 \\
\hline Median RPD for SCo-1 & 9.0 & 2.9 & 1.1 & 6.5 & 0.6 & 0.5 & 2.6 & 4.1 & 6.3 & 2.0 & 1.5 & 0.0 \\
\hline Median RPD for GSD- $\varepsilon$ & 10.5 & 2.6 & 2.5 & 6.9 & 1.9 & 1.1 & 7.8 & 9.1 & 3.4 & 1.0 & 2.9 & 2.3 \\
\hline $\begin{array}{l}\text { Median RPD for MESS-i } \\
\text { MEDIAN RPD FOR ALL SRMS }=1 .\end{array}$ & 3.2 & 2.4 & 1.0 & 2.2 & 0.6 & 2.0 & 0.8 & 3.8 & & 1.6 & 1.2 & 1.4 \\
\hline MAG-1 laboratory & 21.8 & 107 & 30.7 & & 77.7 & 724 & 47.9 & 26.4 & 17.8 & 147 & 138 & 136 \\
\hline MAG-1 literature & 20.4 & 97 & 30.0 & & 79.0 & 760 & 53.0 & 24.0 & 17.2 & 146 & 140 & 130 \\
\hline
\end{tabular}




\begin{tabular}{|c|c|c|c|c|c|c|c|c|c|c|c|c|}
\hline Sample ID & Co, ppm & $\mathrm{Cr}, \mathrm{ppm}$ & $\mathrm{Cu}, \mathrm{ppm}$ & $\mathrm{Hg}, \mathrm{ppm}$ & $\mathrm{Li}, \mathrm{ppm}$ & $\mathrm{Mn}, \mathrm{ppm}$ & $\mathrm{Ni}, \mathrm{ppm}$ & $\mathrm{Pb}, \mathrm{ppm}$ & Sc, ppm & $\mathrm{Sr}, \mathrm{ppm}$ & $\mathrm{V}, \mathrm{ppm}$ & $\mathrm{Zn}, \mathrm{ppm}$ \\
\hline $\begin{array}{r}\text { RPD for each element }= \\
\text { Median RPD for SRM }=\end{array}$ & 6.6 & 9.8 & 2.3 & & 1.7 & 4.9 & 10.1 & 9.5 & 3.4 & 0.7 & 1.4 & 4.5 \\
\hline NIST 2704 laboratory & 13.6 & 143 & 95.7 & & 45.0 & 578 & 41.1 & 156 & 12.3 & 134 & 91.6 & 443 \\
\hline NIST 2704 literature & 14.0 & 135 & 98.6 & & 47.5 & 555 & 44.1 & 161.0 & 12.0 & 130 & 95.0 & 438 \\
\hline $\begin{array}{r}\text { RPD for each element }= \\
\text { Median RPD for SRM }=\end{array}$ & 2.9 & 5.8 & 3.0 & & 5.4 & 4.1 & 7.0 & 3.2 & 2.5 & 3.0 & 3.6 & 1.1 \\
\hline SCo-1 laboratory & 11.2 & 74.6 & 29.5 & & 44.7 & 347 & 25.1 & 31.2 & 12.2 & 166 & 138 & 105 \\
\hline SCo-1 literature & 10.5 & 68.0 & 28.7 & & 45.0 & 410 & 27.0 & 31.0 & 10.8 & 174 & 131 & 103 \\
\hline $\begin{array}{r}\text { RPD for each element }= \\
\text { Median RPD for SRM }=\end{array}$ & 6.5 & 9.3 & 2.7 & & 0.7 & 16.6 & 7.3 & 0.6 & 12.2 & 4.7 & 5.2 & 1.9 \\
\hline GSD-8 laboratory & 3.30 & 6.75 & 5.87 & & 13.2 & 293 & 1.53 & 21.8 & 5.28 & 49.7 & 24.6 & 45.9 \\
\hline GSD-8 literature & 3.6 & 7.60 & 4.1 & & 13.2 & 310 & 2.7 & 21.0 & 5.7 & 52.0 & 26 & 43.0 \\
\hline $\begin{array}{r}\text { RPD for each element }= \\
\text { Median RPD for SRM }=\end{array}$ & 8.7 & 11.8 & 35.5 & & 0.0 & 5.6 & 55.3 & 3.7 & 7.7 & 4.5 & 5.5 & 6.5 \\
\hline MAG-1 laboratory & 21.6 & 109 & 29.1 & & 77.5 & 737 & 46.6 & 27.7 & 17.9 & 151 & 143 & 136 \\
\hline MAG-1 literature & 20.4 & 97 & 30.0 & & 79.0 & 760 & 53.0 & 24.0 & 17.2 & 146 & 140 & 130 \\
\hline $\begin{array}{r}\text { RPD for each element }= \\
\text { Median RPD for SRM }=\end{array}$ & 5.7 & 11.7 & 3.0 & & 1.9 & 3.1 & 12.9 & 14.3 & 4.0 & 3.4 & 2.1 & 4.5 \\
\hline NIST 2704 laboratory & 13.2 & 142 & 92.6 & & 46.1 & 575 & 39.5 & 154 & 11.7 & 132 & 93.2 & 415 \\
\hline NIST 2704 literature & 14.0 & 135 & 98.6 & & 47.5 & 555 & 44.1 & 161.0 & 12.0 & 130 & 95.0 & 438 \\
\hline $\begin{array}{r}\text { RPD for each element }= \\
\text { Median RPD for SRM }=\end{array}$ & 5.9 & 5.1 & 6.3 & & 3.0 & 3.5 & 11.0 & 4.4 & 2.5 & 1.5 & 1.9 & 5.4 \\
\hline SCo-1 laboratory & 11.3 & 77.9 & 28.8 & & 45.6 & 404 & 24.6 & 31.1 & 12.3 & 172 & 141 & 106 \\
\hline SCo-1 literature & 10.5 & 68.0 & 28.7 & & 45.0 & 410 & 27.0 & 31.0 & 10.8 & 174 & 131 & 103 \\
\hline $\begin{array}{r}\text { RPD for each element }= \\
\text { Median RPD for SRM }=\end{array}$ & 7.3 & 13.6 & 0.3 & & 1.3 & 1.5 & 9.3 & 0.3 & 13.0 & 1.2 & 7.4 & 2.9 \\
\hline GSD-8 laboratory & 3.26 & 6.94 & 5.54 & & 12.9 & 336 & 1.62 & 21.8 & 4.73 & 48.5 & 24.0 & 48.1 \\
\hline GSD-8 literature & 3.6 & 7.60 & 4.1 & & 13.2 & 310 & 2.7 & 21.0 & 5.7 & 52.0 & 26 & 43.0 \\
\hline $\begin{array}{r}\text { RPD for each element }= \\
\text { Median RPD for SRM }=\end{array}$ & 9.9 & 9.1 & 29.9 & & 2.3 & 8.0 & 50.0 & 3.7 & 18.6 & 7.0 & 8.0 & 11.2 \\
\hline MAG-1 laboratory & 21.9 & 109 & 30.4 & & 77.0 & 753 & 48.9 & 28.3 & 18.9 & 146 & 146 & 135 \\
\hline MAG-1 literature & 20.4 & 97 & 30.0 & & 79.0 & 760 & 53.0 & 24.0 & 17.2 & 146 & 140 & 130 \\
\hline $\begin{array}{r}\text { RPD for each element }= \\
\text { Median RPD for SRM }=\end{array}$ & 7.1 & 11.7 & 1.3 & & 2.6 & 0.9 & 8.0 & 16.4 & 9.4 & 0.0 & 4.2 & 3.8 \\
\hline NIST 2704 laboratory & 13.5 & 137 & 101 & & 44.8 & 576 & 41.7 & 155 & 12.4 & 134 & 93.3 & 420 \\
\hline NIST 2704 literature & 14.0 & 135 & 98.6 & & 47.5 & 555 & 44.1 & 161.0 & 12.0 & 130 & 95.0 & 438 \\
\hline $\begin{array}{r}\text { RPD for each element }= \\
\text { Median RPD for SRM }=\end{array}$ & 3.6 & 1.5 & 2.4 & & 5.9 & 3.7 & 5.6 & 3.8 & 3.3 & 3.0 & 1.8 & 4.2 \\
\hline SCo-1 laboratory & 11.5 & 74.8 & 30.4 & & 44.7 & 385 & 25.6 & 32.0 & 13.1 & 171 & 139 & 108 \\
\hline SCo-1 literature & 10.5 & 68.0 & 28.7 & & 45.0 & 410 & 27.0 & 31.0 & 10.8 & 174 & 131 & 103 \\
\hline $\begin{array}{r}\text { RPD for each element }= \\
\text { Median RPD for SRM }=\end{array}$ & 9.1 & 9.5 & 5.8 & & 0.7 & 6.3 & 5.3 & 3.2 & 19.2 & 1.7 & 5.9 & 4.7 \\
\hline GSD-8 laboratory & 3.28 & 6.34 & 5.84 & & 13.1 & 324 & 1.58 & 22.5 & 5.35 & 48.5 & 24.4 & 46.7 \\
\hline GSD-8 literature & 3.6 & 7.60 & 4.1 & & 13.2 & 310 & 2.7 & 21.0 & 5.7 & 52.0 & 26 & 43.0 \\
\hline $\begin{array}{r}\text { RPD for each element }= \\
\text { Median RPD for SRM }=\end{array}$ & 9.3 & 18.1 & 35.0 & & 0.8 & 4.4 & 52.3 & 6.9 & 6.3 & 7.0 & 6.3 & 8.2 \\
\hline MAG-1 laboratory & 21.7 & 112 & 30.4 & & 77.7 & 772 & 48.1 & 27.5 & 18.4 & 142 & 151 & 134 \\
\hline
\end{tabular}




\begin{tabular}{|c|c|c|c|c|c|c|c|c|c|c|c|c|}
\hline Sample ID & Co, ppm & $\mathrm{Cr}, \mathrm{ppm}$ & $\mathrm{Cu}, \mathrm{ppm}$ & $\mathrm{Hg}, \mathrm{ppm}$ & $\mathrm{Li}, \mathrm{ppm}$ & $\mathrm{Mn}, \mathrm{ppm}$ & $\mathrm{Ni}, \mathrm{ppm}$ & $\mathrm{Pb}, \mathrm{ppm}$ & Sc, ppm & $\mathrm{Sr}, \mathrm{ppm}$ & $\mathrm{V}, \mathrm{ppm}$ & $\mathrm{Zn}, \mathrm{ppm}$ \\
\hline MAG-1 literature & 20.4 & 979 & $=30.0$ & & 7979.0 & 7670 & $=53.0$ & 24.0 & 1717.2 & 10146 & 10140 & 13130 \\
\hline $\begin{array}{l}\text { RPD for each element = } \\
\text { Median RPD for SRM = }\end{array}$ & 6.2 & 14.4 & 1.3 & & 1.7 & 1.6 & 9.7 & 13.6 & 6.7 & 2.8 & 7.6 & 3.0 \\
\hline NIST 2704 laboratory & 13.6 & 145 & 101 & & 46.1 & 608 & 40.8 & 148 & 12.2 & 130 & 99.1 & 424 \\
\hline NIST 2704 literature & 14.0 & 135 & 98.6 & & 47.5 & 555 & 44.1 & 161.0 & 12.0 & 130 & 95.0 & 438 \\
\hline $\begin{array}{r}\text { RPD for each element }= \\
\text { Median RPD for SRM = }\end{array}$ & 2.9 & 7.1 & 2.4 & & 3.0 & 9.1 & 7.8 & 8.4 & 1.7 & 0.0 & 4.2 & 3.2 \\
\hline SCo-1 laboratory & 11.2 & 80.3 & 30.9 & & 46.4 & 398 & 24.9 & 30.9 & 12.9 & 166 & 149 & 107 \\
\hline SCo-1 literature & 10.5 & 68.0 & 28.7 & & 45.0 & 410 & 27.0 & 31.0 & 10.8 & 174 & 131 & 103 \\
\hline $\begin{array}{r}\text { RPD for each element }= \\
\text { Median RPD for SRM }=\end{array}$ & 6.5 & 16.6 & 7.4 & & 3.1 & 3.0 & 8.1 & 0.3 & 17.7 & 4.7 & 12.9 & 3.8 \\
\hline GSD-8 laboratory & 3.36 & 7.16 & 6.01 & & 13.7 & 338 & 1.46 & 21.5 & 5.32 & 47.0 & 26.6 & 46.6 \\
\hline GSD-8 literature & 3.6 & 7.60 & 4.1 & & 13.2 & 310 & 2.7 & 21.0 & 5.7 & 52.0 & 26 & 43.0 \\
\hline $\begin{array}{r}\text { RPD for each element }= \\
\text { Median RPD for SRM }=\end{array}$ & 6.9 & 6.0 & 37.8 & & 3.7 & 8.6 & 59.6 & 2.4 & 6.9 & 10.1 & 2.3 & 8.0 \\
\hline MAG-1 laboratory & 22.4 & 114 & 31.0 & & 78.3 & 768 & 49.4 & 19.4 & 19.2 & 148 & 155 & 142 \\
\hline MAG-1 literature & 20.4 & 97 & 30.0 & & 79.0 & 760 & 53.0 & 24.0 & 17.2 & 146 & 140 & 130 \\
\hline $\begin{array}{r}\text { RPD for each element }= \\
\text { Median RPD for SRM = }\end{array}$ & 9.3 & 16.1 & 3.3 & & 0.9 & 1.0 & 7.0 & 21.2 & 11.0 & 1.4 & 10.2 & 8.8 \\
\hline NIST 2704 laboratory & 13.8 & 146 & 103 & & 45.2 & 581 & 42.4 & 158 & 12.8 & 133 & 99.8 & 447 \\
\hline NIST 2704 literature & 14.0 & 135 & 98.6 & & 47.5 & 555 & 44.1 & 161.0 & 12.0 & 130 & 95.0 & 438 \\
\hline $\begin{array}{l}\text { RPD for each element = } \\
\text { Median RPD for SRM = }\end{array}$ & 1.4 & 7.8 & 4.4 & & 5.0 & 4.6 & 3.9 & 1.9 & 6.5 & 2.3 & 4.9 & 2.0 \\
\hline SCO-1 laboratory & 11.5 & 77.8 & 29.8 & & 44.0 & 403 & 25.6 & 29.8 & 13.2 & 174 & 145 & 110 \\
\hline SCO-1 literature & 10.5 & 68.0 & 28.7 & & 45.0 & 410 & 27.0 & 31.0 & 10.8 & 174 & 131 & 103 \\
\hline $\begin{array}{r}\text { RPD for each element }= \\
\text { Median RPD for SRM }=\end{array}$ & 9.1 & 13.4 & 3.8 & & 2.2 & 1.7 & 5.3 & 3.9 & 20.0 & 0.0 & 10.1 & 6.6 \\
\hline GSD-8 laboratory & 3.27 & 6.38 & 5.83 & & 12.3 & 334 & $<1$ & 19.6 & 5.38 & 45.6 & 24.0 & 47.3 \\
\hline GSD-8 literature & 3.6 & 7.60 & 4.1 & & 13.2 & 310 & 2.7 & 21.0 & 5.7 & 52.0 & 26 & 43.0 \\
\hline $\begin{array}{l}\text { RPD for each element }= \\
\text { Median RPD for SRM }=\end{array}$ & 9.6 & 17.5 & 34.8 & & 7.1 & 7.5 & & 6.9 & 5.8 & 13.1 & 8.0 & 9.5 \\
\hline MAG-1 laboratory & 22.2 & 107 & 30.5 & & 78.6 & 742 & 48.4 & 28.2 & 18.7 & 150 & 145 & 138 \\
\hline MAG-1 literature & 20.4 & 97 & 30.0 & & 79.0 & 760 & 53.0 & 24.0 & 17.2 & 146 & 140 & 130 \\
\hline $\begin{array}{l}\text { RPD for each element = } \\
\text { Median RPD for SRM = }\end{array}$ & 8.5 & 9.8 & 1.7 & & 0.5 & 2.4 & 9.1 & 16.1 & 8.4 & 2.7 & 3.5 & 6.0 \\
\hline NIST 2704 laboratory & 13.5 & 138 & 95.8 & & 45.7 & 570 & 41.6 & 158 & 12.7 & 136 & 94.2 & 446 \\
\hline NIST 2704 literature & 14.0 & 135 & 98.6 & & 47.5 & 555 & 44.1 & 161.0 & 12.0 & 130 & 95.0 & 438 \\
\hline $\begin{array}{r}\text { RPD for each element }= \\
\text { Median RPD for SRM }=\end{array}$ & 3.6 & 2.2 & 2.9 & & 3.9 & 2.7 & 5.8 & 1.9 & 5.7 & 4.5 & 0.8 & 1.8 \\
\hline SCo-1 laboratory & 11.4 & 75.0 & 29.7 & & 45.1 & 390 & 25.9 & 32.0 & 13.0 & 172 & 139 & 111 \\
\hline SCo-1 literature & 10.5 & 68.0 & 28.7 & & 45.0 & 410 & 27.0 & 31.0 & 10.8 & 174 & 131 & 103 \\
\hline $\begin{array}{l}\text { RPD for each element = } \\
\text { Median RPD for SRM = }\end{array}$ & 8.2 & 9.8 & 3.4 & & 0.2 & 5.0 & 4.2 & 3.2 & 18.5 & 1.2 & 5.9 & 7.5 \\
\hline GSD-8 laboratory & 3.28 & 6.15 & 5.22 & & 12.9 & 327 & 1.22 & 21.5 & 5.44 & 47.4 & 23.9 & 45.4 \\
\hline GSD-8 literature & 3.6 & 7.60 & 4.1 & & 13.2 & 310 & 2.7 & 21.0 & 5.7 & 52.0 & 26 & 43.0 \\
\hline $\begin{array}{r}\text { RPD for each element }= \\
\text { Median RPD for SRM }=\end{array}$ & 9.3 & 21.1 & 24.0 & & 2.3 & 5.3 & 75.5 & 2.4 & 4.7 & 9.3 & 8.4 & 5.4 \\
\hline
\end{tabular}




\begin{tabular}{|c|c|c|c|c|c|c|c|c|c|c|c|c|}
\hline Sample ID & Co, ppm & $\mathrm{Cr}, \mathrm{ppm}$ & $\mathrm{Cu}, \mathrm{ppm}$ & $\mathrm{Hg}, \mathrm{ppm}$ & $\mathrm{Li}, \mathrm{ppm}$ & $\mathrm{Mn}, \mathrm{ppm}$ & $\mathrm{Ni}, \mathrm{ppm}$ & $\mathrm{Pb}, \mathrm{ppm}$ & $\mathrm{Sc}, \mathrm{ppm}$ & $\mathrm{Sr}, \mathrm{ppm}$ & V, ppm & $\mathrm{Zn}, \mathrm{ppm}$ \\
\hline MAG-1 laboratory & 222.1 & 1110 & 30.1 & & 80.5 & $=766.0$ & $=49.4$ & 224.9 & 1019.3 & $=138$ & 10 & 13138 \\
\hline MAG-1 literature & 20.4 & 97 & 30.0 & & 79.0 & 760 & 53.0 & 24.0 & 17.2 & 146 & 140 & 130 \\
\hline $\begin{array}{r}\text { RPD for each element }= \\
\text { Median RPD for SRM }=\end{array}$ & 8.0 & 12.1 & 0.4 & & 1.9 & 0.8 & 7.1 & 3.8 & 11.4 & 5.6 & 6.7 & 5.6 \\
\hline NIST 2704 laboratory & 13.8 & 139 & 95.1 & & 47.6 & 571.0 & 42.4 & 165.0 & 12.6 & 125 & 95.6 & 434 \\
\hline NIST 2704 literature & 14.0 & 135 & 98.6 & & 47.5 & 555 & 44.1 & 152 & 12.0 & 130 & 95.0 & 438 \\
\hline $\begin{array}{r}\text { RPD for each element }= \\
\text { Median RPD for SRM }=\end{array}$ & 1.4 & 2.6 & 3.7 & & 0.1 & 2.8 & 4.0 & 8.2 & 4.9 & 3.9 & 0.6 & 0.9 \\
\hline Sco-1 laboratory & 11.6 & 74.1 & 29.9 & & 46.7 & 393.0 & 25.8 & 35.6 & 12.8 & 164 & 140 & 109 \\
\hline SCO-1 literature & 10.5 & 68.0 & 28.7 & & 45.0 & 410 & 27.0 & 31.0 & 10.8 & 174 & 131 & 103 \\
\hline $\begin{array}{r}\text { RPD for each element }= \\
\text { Median RPD for SRM }=\end{array}$ & 10.0 & 8.5 & 3.9 & & 3.7 & 4.2 & 4.7 & 13.8 & 16.9 & 5.9 & 6.6 & 5.7 \\
\hline GSD-8 laboratory & 3.3 & 6.4 & 5.8 & & 13.6 & 338.50 & 1.4 & 21.6 & 5.5 & 46 & 24.6 & 47.3 \\
\hline GSD-8 literature & 3.60 & 7.60 & 4.10 & & 13.2 & 310 & 2.70 & 21.0 & 5.54 & 52.0 & 26 & 43.0 \\
\hline $\begin{array}{r}\text { RPD for each element }= \\
\text { Median RPD for SRM }=\end{array}$ & 9.2 & 17.5 & 35.0 & & 2.6 & 8.8 & 61.5 & 2.6 & 0.5 & 13.3 & 5.7 & 9.5 \\
\hline MAG-1 laboratory & 22.6 & 114 & 29.2 & & 78.8 & 798 & 50.2 & 17.6 & 19.1 & 144 & 152 & 138 \\
\hline MAG-1 literature & 20.4 & 97 & 30.0 & & 79.0 & 760 & 53.0 & 24.0 & 17.2 & 146 & 140 & 130 \\
\hline $\begin{array}{r}\text { RPD for each element }= \\
\text { Median RPD for SRM }=\end{array}$ & 10.2 & 16.1 & 2.7 & & 0.3 & 4.9 & 5.4 & 30.8 & 10.5 & 1.4 & 8.2 & 6.0 \\
\hline NIST 2704 laboratory & 13.6 & 144 & 97.5 & & 45.7 & 600 & 42.3 & 156 & 12.5 & 132 & 94.6 & 438 \\
\hline NIST 2704 literature & 14.0 & 135 & 98.6 & & 47.5 & 555 & 44.1 & 161.0 & 12.0 & 130 & 95.0 & 438 \\
\hline $\begin{array}{r}\text { RPD for each element }= \\
\text { Median RPD for SRM }=\end{array}$ & 2.9 & 6.5 & 1.1 & & 3.9 & 7.8 & 4.2 & 3.2 & 4.1 & 1.5 & 0.4 & 0.0 \\
\hline SCo-1 laboratory & 11.9 & 79.1 & 37.9 & & 47.0 & 431 & 26.5 & 31.7 & 13.0 & 176 & 145 & 111 \\
\hline SCo-1 literature & 10.5 & 68.0 & 28.7 & & 45.0 & 410 & 27.0 & 31.0 & 10.8 & 174 & 131 & 103 \\
\hline $\begin{array}{r}\text { RPD for each element }= \\
\text { Median RPD for SRM }=\end{array}$ & 12.5 & 15.1 & 27.6 & & 4.3 & 5.0 & 1.9 & 2.2 & 18.5 & 1.1 & 10.1 & 7.5 \\
\hline GSD-8 laboratory & 3.48 & 6.28 & 5.07 & & 13.5 & 367 & 1.04 & 21.1 & 5.54 & 47.1 & 25.2 & 47.8 \\
\hline GSD-8 literature & 3.6 & 7.60 & 4.1 & & 13.2 & 310 & 2.7 & 21.0 & 5.7 & 52.0 & 26 & 43.0 \\
\hline $\begin{array}{r}\text { RPD for each element }= \\
\text { Median RPD for SRM }=\end{array}$ & 3.4 & 19.0 & 21.2 & & 2.2 & 16.8 & 88.8 & 0.5 & 2.8 & 9.9 & 3.1 & 10.6 \\
\hline MAG-1 laboratory & 22.5 & 114 & 30.0 & & 81.2 & 790 & 49.0 & 20.5 & 19.0 & 146 & 150 & 138 \\
\hline MAG-1 literature & 20.4 & 97 & 30.0 & & 79.0 & 760 & 53.0 & 24.0 & 17.2 & 146 & 140 & 130 \\
\hline $\begin{array}{r}\text { RPD for each element }= \\
\text { Median RPD for SRM }=\end{array}$ & 9.8 & 16.1 & 0.0 & & 2.7 & 3.9 & 7.8 & 15.7 & 9.9 & 0.0 & 6.9 & 6.0 \\
\hline NIST 2704 laboratory & 13.9 & 145 & 95.0 & & 47.0 & 591 & 42.0 & 151 & 12.6 & 134 & 97.6 & 431 \\
\hline NIST 2704 literature & 14.0 & 135 & 98.6 & & 47.5 & 555 & 44.1 & 161.0 & 12.0 & 130 & 95.0 & 438 \\
\hline $\begin{array}{r}\text { RPD for each element }= \\
\text { Median RPD for SRM }=\end{array}$ & 0.7 & 7.1 & 3.7 & & 1.1 & 6.3 & 4.9 & 6.4 & 4.9 & 3.0 & 2.7 & 1.6 \\
\hline SCo-1 laboratory & 11.8 & 79.4 & 29.1 & & 47.2 & 420 & 25.9 & 31.4 & 13.1 & 173 & 144 & 109 \\
\hline SCo-1 literature & 10.5 & 68.0 & 28.7 & & 45.0 & 410 & 27.0 & 31.0 & 10.8 & 174 & 131 & 103 \\
\hline $\begin{array}{r}\text { RPD for each element }= \\
\text { Median RPD for SRM }=\end{array}$ & 11.7 & 15.5 & 1.4 & & 4.8 & 2.4 & 4.2 & 1.3 & 19.2 & 0.6 & 9.5 & 5.7 \\
\hline GSD-8 laboratory & 3.51 & 5.98 & 4.59 & & 13.8 & 353 & 1.41 & 21.2 & 5.49 & 49.3 & 24.8 & 49.9 \\
\hline GSD-8 literature & 3.6 & 7.60 & 4.1 & & 13.2 & 310 & 2.7 & 21.0 & 5.7 & 52.0 & 26 & 43.0 \\
\hline $\begin{array}{r}\text { RPD for each element }= \\
\text { Median RPD for SRM }=\end{array}$ & 2.5 & 23.9 & 11.3 & & 4.4 & 13.0 & 62.8 & 0.9 & 3.8 & 5.3 & 4.7 & 14.9 \\
\hline
\end{tabular}


Sample ID

$\begin{array}{llllllllllll}\mathrm{Co}, \mathrm{ppm} & \mathrm{Cr}, \mathrm{ppm} & \mathrm{Cu}, \mathrm{ppm} & \mathrm{Hg}, \mathrm{ppm} & \mathrm{Li}, \mathrm{ppm} & \mathrm{Mn}, \mathrm{ppm} & \mathrm{Ni}, \mathrm{ppm} & \mathrm{Pb}, \mathrm{ppm} & \mathrm{Sc}, \mathrm{ppm} & \mathrm{Sr}, \mathrm{ppm} & \mathrm{V}, \mathrm{ppm} & \mathrm{Zn}, \mathrm{ppm}\end{array}$

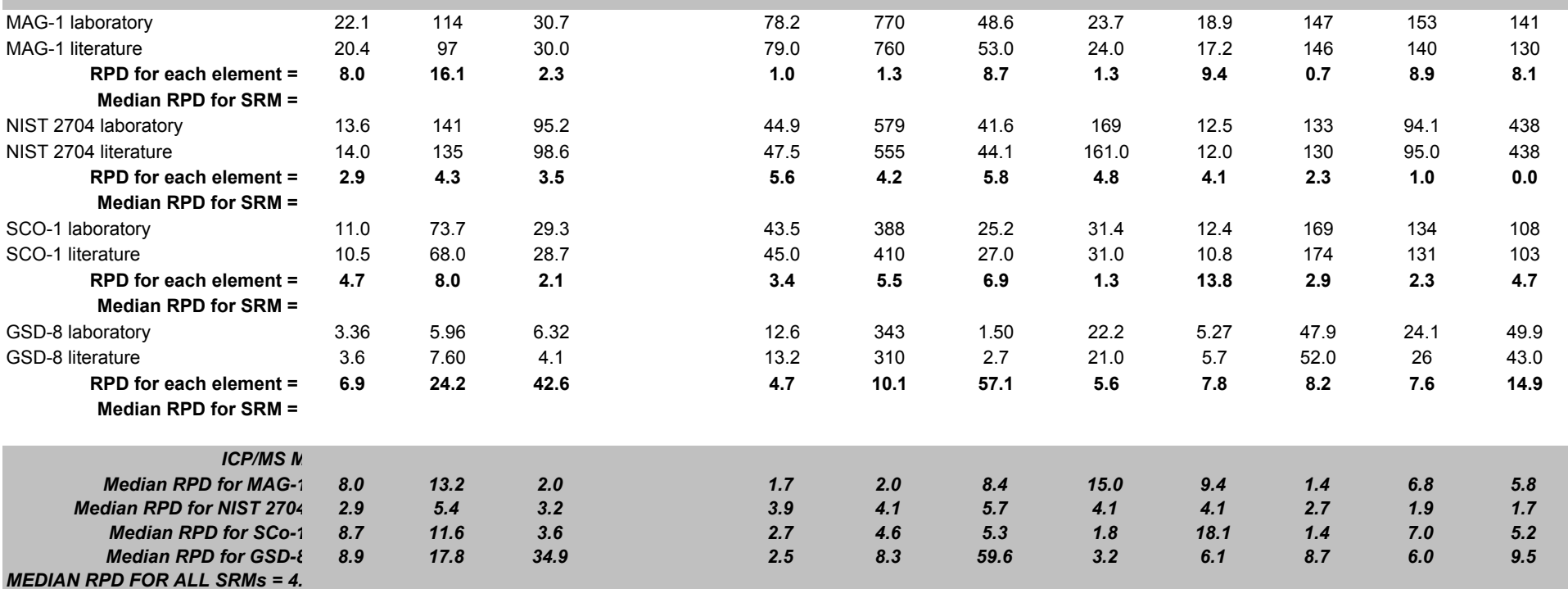


Table B7. Sample groups for major and trace elements and forms of carbon analyses and duplicate and triplicate sample relative percent differences

Environ. Duplicate

samples

\section{Samples grouped for analysis Job number Range and media \\ RPD}

All Upper Mystic Lake core MYS.B2
samples

none

All Harris Pond core HSP.1 samples none

$0.0-45.7 \%, 3.9 \%$

All Harris Pond core HSP.B2

samples

All Harris Pond core HSP.B3

samples

FOS. 4 0-5, 5-10, 10-15, 20-25, 30-

$35,45-50$

FOS. 4 55-60, 70-75, 85-90, 100-

$105,105-112$; FOS $20-10,50-60$

100-110; FOS 5 0-10, 50-60, 80-90

Sites 1 and 6 (03/08/01): Site 4

(03/09/01)

All Echo Lake samples

Round Top Brook (01/10/2001):

Charles River (03/28/2001); Mill

River (03/28/2001); Sites 1, 3, 4, 5

and $6(10 / 15 / 00)$; Sycamore \#2

(05/04/01); Sycamore \#4\&5 and

he7 (n5/n5/n1)

Composite \#1 and

2 (03/27/2001); Aberjona River

(03/22/2001), and \#3 (03/24/2001):

Site $2(10 / 15 / 01)$; $B$ (03/24/200

Site 2 (10/15/01); Big Fossi

(05/25/2001): Composite

(05/25/2001); Mill River

$0.0-9.2 \%, 2.4 \%$

$0.0-9.8 \%, 2.4 \%$

$0.0-8.4 \%, 1.3 \%$

3210

$0.0-15.4 \%, 2.1 \%$

3342

\section{SRMs}

Range and median RPD

Method

ICP/AES

NIST 2704: $0.0-1$

(C) $1: 0.0-10.2 \%, 1.0 \%$

SCo-1: $0.0-10.2 \%, 1.6 \%$

GSD-8. $0.0-11.8 \%, 2.6 \%$

MAG-1: $0.0-8.4 \%, 2.0 \%$

NIST 2704: $0.0-19.9 \%, 2.6 \%$

SCo-1: $0.0-13.3 \%, 2.3 \%$

GSD-8: $0.1-16.7 \%, 2.3 \%$

MESS-2: $\quad 0.0-8.7 \%, 1.0 \%$

MAG-1: $0.0-50.7 \%, 1.9 \%$

NIST 2704: $0.0-6.8 \%, 1.8 \%$ SCo-1: $0.0-54.0 \%, 1.5 \%$

GSD-8: $0.0-31.6 \%, 2.4 \%$

MESS-2: $0.0-4.9 \%, 0.9 \%$

NIST 2704: $0.2-31.7 \%, 2.5 \%$

NIST 2704. 0.2-31.7\%, $2.5 \%$

SCo-1: $0.0-29.6 \%, 1.7 \%$

GSD-8: $0.0-40.5 \%, 2.7 \%$

MESS-2: $0.2-8.7 \%, 2.5 \%$

NIST 2704: $0.0-41.7 \%, 3.0 \%$

SCo-1: $0.1-16.6 \%, 3.8 \%$

SCo-1: $0.1-16.6 \%, 3.8 \%$

GSD-8: $0.0-97.2 \%, 5.6 \%$

NIST 2704: $0.0-41.7 \%, 3.0 \%$

IST 2704: $0.0-41.7 \%, 3.0 \%$

SCo-1: $0.1-16.6 \%, 3.8 \%$

GSD-8: $0.0-97.2 \%, 5.6 \%$

MAG-1: $0.1-50.9 \%, 3.7 \%$

NIST 2704. 0.0-41.7\%, $4.6 \%$

SCo-1: $0.3-21.1 \%, 3.8 \%$

GSD-8: $0.4-50.0 \%, 8.1 \%$

MAG-1: $0.1-50.9 \%, 3.7 \%$

NIST 2704. 0.0-41.7\%, $4.6 \%$

SCo-1: $0.3-21.1 \%, 3.8 \%$

GSD-8: $0.4-50.0 \%, 8.1 \%$

MAG-1: $0.0-57.7 \%, 4.1 \%$

NIST 2704: $0.5-36.4 \%, 3.0 \%$

SCo-1: $0.0-23.3 \%, 4.7 \%$

GSD-8: $0.0-52.3 \%, 6.9 \%$

MAG-1: $0.4-48.1 \%, 3.0 \%$

NIST 2704: $0.0-32.5 \%, 3.3 \%$

SCo-1: $0.3-26.1 \%, 6.5 \%$

GSD-8: $0.3-59.6 \%, 8.0 \%$

$3.2-24.9 \%, 16.4 \% \quad$ MAG $1: 0.3-21.2 \%, 7.1 \%$

NIST 2704: $1.4-34.0 \%, 4.1 \%$
ICPIAES

ICP/AES

ICP/AES

ICP/MS

ICP/MS

ICP/MS

ICP/MS

ICP/MS

ICP/MS

ICP/MS 
Table B7. Sample groups for major and trace elements and forms of carbon analyses and duplicate and triplicate sample relative percent differences

Environ. Duplicate samples

Samples grouped for analysis Job number

Range and median

RPD

Range and median RPD

Method

(05/29/2001); Site 5 (05/04/01); Site

5 duplicate (05/04/01); Sycamore

$\# 1$ or \#3? (05/04/01)

All Lake Como samples

3427

$0.0-14.6 \%, 2.2 \%$

SD-8: $1.4-82.2 \%, 10.5 \%$

MAG-1: $0.3-21.2 \%, 7.1 \%$

NIST 2704: $1.4-34.0 \%, 4.1 \%$

SCo-1: $0.0-20.0 \%, 4.7 \%$

$0.0-10.5 \%, 2.7 \%$ MAG-1: $0.5-16.1 \%, 6.0 \%$

NIST 2704: $0.0-34.6 \%, 2.4 \%$

SCo-1: $0.2-18.5 \%, 4.0 \%$

GSD-8: $0.8-75.5 \%, 5.8 \%$

09/20/01); Site 5 (08/16/01); Site 5

duplicate (08/16/01); Sycamore \#

Big Fossil (08/11/01, 09/18/0

10/11/01); Fosdic Lake Inflow

(08/11/01, 09/18/01, 10/10/01)

Lake Como Inflow (08/30/01,

09/20/01, 11/09/01); Sycamore \#2

(08/16/01); Sycamore \#1, 2, 3, 4,

and 5 (08/30/01); Echo Lake Inflow

Fosdic Lake Inflow (12/16/01)

Lake Como Inflow (01/23/02)

Aberjona River Composite and lab.

dup. (09/05/2002)

ALL JOBS IN STUDY

\section{ALL JOBS IN STUDY}

3784

3818

4315

$9.0-16.2 \%, 11.8 \%$

MAG-1: $0.4-20.1 \%, 5.6 \%$

NIST 2704: $0.1-38.9 \%, 3.1 \%$

SCo-1: $0.0-16.9 \%, 5.6 \%$

GSD-8: $0.1-61.5 \%, 8.8 \%$

$0.0-6.2 \%, 1.0 \% \quad$ MAG-1: $0.3-30.8 \%, 6.5 \%$

NIST 2704: $0.0-45.6 \%, 3.0 \%$

SCo-1: $0.0-27.6 \%, 6.0 \%$

GSD-8: $0.5-88.8 \%, 8.8 \%$

$0.0-4.0 \%, 1.3 \% \quad$ MAG-1: $0.0-21.2 \%, 6.0 \%$

NIST $2704: 0.0-35.5 \%, 3.3 \%$

SCo-1: $0.2-19.2 \%, 4.7 \%$

GSD-8: $0.9-62.8 \%, 5.9 \%$

$0.0-3.7 \%, 0.5 \% \quad$ MAG-1: $0.7-22.8 \%, 3.9 \%$

NIST $2704: 0.0-38.2 \%, 3.2 \%$

SCo-1: $0.2-13.8 \%, 3.0 \%$

GSD-8: $0.8-68.2 \%, 7.6 \%$

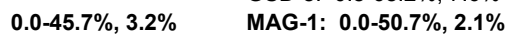

NIST 2704: $0.0-31.7 \%, 1.8 \%$

SCo-1: $0.0-54.0 \%, 1.8 \%$

GSD-8: $0.0-40.5 \%, 2.5 \%$

MESS-2: $0.0-8.7 \%, 1.6 \%$

All SRMs: $0.0-54.0 \%, 1.9 \%$

NIST 2704: $0.0-45.6 \%, 3.2 \%$

SCO-1: $0.0-27.6 \%, 4.7 \%$

GSD-8: $0.0-97.2 \%, 7.8 \%$

All SRM: $0.0-97.2 \%, 4.7 \%$
MAG-1: $0.0-57.7 \%, 5.1 \%$
ICP/MS

ICP/MS

ICP/MS

ICP/MS

ICP/MS

ICP/MS

ICPIAES

ICP/MS 
Table B8. Major and trace element duplicate and triplicate samples.

\begin{tabular}{|c|c|c|c|c|c|c|c|c|c|c|}
\hline Sample ID & Job No. & Method & O.C., \% & Al, $\mu \mathrm{g} / \mathrm{g}$ & $\mathrm{Ca}, \mu \mathrm{g} / \mathrm{g}$ & $\mathrm{Fe}, \mu \mathrm{g} / \mathrm{g}$ & $\mathrm{K}, \mu \mathrm{g} / \mathrm{g}$ & $\mathbf{M g}, \mu \mathbf{g} / \mathbf{g}$ & $\mathrm{Na}, \mu \mathbf{g} / \mathbf{g}$ & $P, \mu g / g$ \\
\hline$\overline{\text { MYS.B2 3-4 }}$ & none & ICP/AES & na & 76,000 & 14,000 & 47,889 & 15,833 & 11,556 & 10,353 & 2,300 \\
\hline MYS.B2 3-4 Dup & none & ICP/AES & na & 75,500 & 13,889 & 47,778 & 15,833 & 11,111 & 10,235 & 2,300 \\
\hline $\begin{array}{l}\text { RPD for each compound: } \\
\text { Median RPD of sample }=1.1\end{array}$ & & & & 0.7 & 0.8 & 0.2 & 0.0 & 3.9 & 1.1 & 0.0 \\
\hline MYS.B2 12-13 & none & ICP/AES & na & 76,900 & 13,636 & 50,111 & 12,100 & 9,800 & 9,778 & 2,300 \\
\hline MYS.B2 12-13 Dup & none & ICP/AES & na & 76,167 & 13,636 & 50,778 & 12,750 & 10,000 & 10,000 & 2,200 \\
\hline $\begin{array}{l}\text { RPD for each compound: } \\
\text { Median RPD of sample }=1.5\end{array}$ & & & & 1.0 & 0.0 & 1.3 & 5.2 & 2.0 & 2.2 & 4.4 \\
\hline MYS.B2 28-30 & none & ICP/AES & na & 85,100 & 14,000 & 45,111 & 12,125 & 13,667 & 10,444 & 2,100 \\
\hline MYS.B2 28-30 Dup & none & ICP/AES & na & 83,636 & 13,412 & 45,111 & 11,875 & 11,667 & 10,111 & 2,200 \\
\hline $\begin{array}{l}\text { RPD for each compound: } \\
\text { Median RPD of sample }=2.1\end{array}$ & & & & 1.7 & 4.3 & 0.0 & 2.1 & 15.8 & 3.2 & 4.7 \\
\hline HSP.1 18-21 & none & ICP/AES & 8.7 & 82,182 & 16,500 & 21,778 & 11,294 & 6,571 & 14,750 & 1,500 \\
\hline HSP.1 18-21 Dup & none & ICP/AES & 8.8 & 97,455 & 17,200 & 21,300 & 11,176 & 7,100 & 15,750 & 1,400 \\
\hline HSP.1 30-33 & none & ICP/AES & 2.7 & 72,900 & 10,300 & 16,333 & 13,882 & 4,100 & 16,375 & 280 \\
\hline HSP.1 30-33 Dup & none & ICP/AES & 1.7 & 70,182 & 10,500 & 14,900 & 13,412 & 3,429 & 15,875 & 280 \\
\hline $\begin{array}{l}\text { RPD for each compound: } \\
\text { Median RPD of sample }=7.0\end{array}$ & & & 45.7 & 3.8 & 1.9 & 9.2 & 3.4 & 17.8 & 3.1 & 0.0 \\
\hline HSP.B2 4-5 & none & ICP/AES & 10.2 & 81,833 & 14,857 & 33,294 & 12,588 & 10,667 & 14,533 & 2,000 \\
\hline HSP.B2 4-5 Dup & none & ICP/AES & 10.6 & 79,200 & 12,857 & 31,412 & 12,235 & 10,000 & 13,733 & 1,900 \\
\hline $\begin{array}{l}\text { RPD for each compound: } \\
\text { Median RPD of sample }=4.4\end{array}$ & & & 3.4 & 3.3 & 14.4 & 5.8 & 2.8 & 6.5 & 5.7 & 5.1 \\
\hline HSP.B2 8-9 & none & ICP/AES & 9.3 & 84,000 & 13,700 & 33,100 & 13,600 & 10,500 & 14,700 & 2,200 \\
\hline HSP.B2 8-9 Dup & none & ICP/AES & 9.2 & 77,000 & 11,857 & 31,059 & 12,941 & 9,000 & 14,000 & 1,800 \\
\hline $\begin{array}{l}\text { RPD for each compound: } \\
\text { Median RPD of sample }=8.1\end{array}$ & & & 1.4 & 8.7 & 14.4 & 6.4 & 5.0 & 15.4 & 4.9 & 20.0 \\
\hline HSP.B3 6-9 & none & ICP/AES & 9.3 & 81,818 & 17,636 & 23,882 & 14,118 & 9,000 & 16,750 & 1,500 \\
\hline
\end{tabular}


Sample ID

Job No.

Method

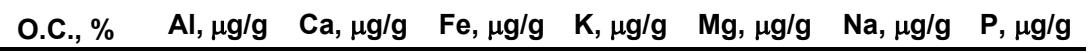

\begin{tabular}{|c|c|c|c|c|c|c|c|c|c|c|}
\hline HSP.B3 21-24 & none & ICP/AES & 7.4 & 73,636 & 18,909 & 24,235 & 14,588 & 9,000 & 17,875 & 1,200 \\
\hline HSP.B3 21-24 Dup & none & ICP/AES & 7.4 & 81,636 & 19,455 & 24,588 & 15,059 & 9,667 & 18,500 & 1,300 \\
\hline RPD for each compound: & & & 0.1 & 10.3 & 2.8 & 1.4 & 3.2 & 7.1 & 3.4 & 8.0 \\
\hline
\end{tabular}

for each compound:

Median RPD of sample $=3.4$

\begin{tabular}{|c|c|c|c|c|c|c|c|c|c|c|}
\hline \multicolumn{3}{|c|}{ ICP/AES METHOD } & \multirow[b]{2}{*}{1.7} & \multirow[b]{2}{*}{3.3} & \multirow[b]{2}{*}{2.8} & \multirow[b]{2}{*}{1.4} & \multirow[b]{2}{*}{2.8} & \multirow[b]{2}{*}{7.7} & \multirow[b]{2}{*}{3.2} & \multirow[b]{2}{*}{5.1} \\
\hline $\begin{array}{r}\text { MEDIAN RI } \\
\text { MEDIAN RPD FOR ALL JO }\end{array}$ & $\begin{array}{l}\text { MPOUI } \\
\text { JNDS = }\end{array}$ & & & & & & & & & \\
\hline FOS.4 0-5 & 3186 & ICP/MS & 2.67 & 51,000 & 94,600 & 29,900 & 7,670 & 4,430 & 1,050 & 1,620 \\
\hline FOS.4 0-5 rep. 1 & 3186 & ICP/MS & na & 48,900 & 86,300 & 27,900 & 6,660 & 4,570 & 1,060 & 1,480 \\
\hline \multicolumn{10}{|l|}{ Median RPD of sample $=6.5$} & 9.0 \\
\hline FOS.4 0-5 rep. 2 & 3186 & ICP/MS & na & 48,200 & 83,900 & 27,300 & 6,490 & 4,630 & 1,060 & 1,450 \\
\hline \multicolumn{11}{|l|}{ Median RPD of sample = 8.4} \\
\hline FOS.4 0-5 rep. 1 & 3186 & ICP/MS & na & 48,900 & 86,300 & 27,900 & 6,660 & 4,570 & 1,060 & 1,480 \\
\hline FOS.4 0-5 rep. 2 & 3186 & ICP/MS & na & 48,200 & 83,900 & 27,300 & 6,490 & 4,630 & 1,060 & 1,450 \\
\hline $\begin{array}{l}\text { RPD for each compound: } \\
\text { Median RPD of sample }=2.0\end{array}$ & & & & 1.4 & 2.8 & 2.2 & 2.6 & 1.3 & 0.0 & 2.0 \\
\hline FOS. $455-60$ rep. 1 & 3188 & ICP/MS & na & 60,900 & 93,000 & 31,300 & 8,150 & 5,090 & 1,060 & 784 \\
\hline $\begin{array}{l}\text { RPD for each compound: } \\
\text { Median RPD of sample }=1.0\end{array}$ & & & & 1.2 & 0.4 & 1.0 & 0.0 & 1.0 & 0.9 & 0.4 \\
\hline FOS.4 55-60 & 3188 & ICP/MS & 2.97 & 60,200 & 93,400 & 31,000 & 8,150 & 5,140 & 1,070 & 781 \\
\hline FOS. $455-60$ rep. 2 & 3188 & $\mathrm{ICP} / \mathrm{MS}$ & na & 60,500 & 92,900 & 31,000 & 8,140 & 5,080 & 1,060 & 775 \\
\hline $\begin{array}{l}\text { RPD for each compound: } \\
\text { Median RPD of sample }=0.9\end{array}$ & & & & 0.5 & 0.5 & 0.0 & 0.1 & 1.2 & 0.9 & 0.8 \\
\hline FOS.4 55-60 rep. 1 & 3188 & ICP/MS & na & 60,900 & 93,000 & 31,300 & 8,150 & 5,090 & 1,060 & 784 \\
\hline FOS.4 55-60 rep. 2 & 3188 & ICP/MS & na & 60,500 & 92,900 & 31,000 & 8,140 & 5,080 & 1,060 & 775 \\
\hline $\begin{array}{l}\text { RPD for each compound: } \\
\text { Median RPD of sample }=0.8\end{array}$ & & & & 0.7 & 0.1 & 1.0 & 0.1 & 0.2 & 0.0 & 1.2 \\
\hline Site 1, Benbrook 03/08/01 @ 1300 & 3201 & ICP/MS & 4.97 & 34,300 & 143,000 & 15,900 & 6,450 & 5,660 & 1,270 & 610 \\
\hline
\end{tabular}


Sample ID

Job No. Method

$\begin{array}{lllllll}\text { O.C., } \% & \text { Al, } \mu \mathrm{g} / \mathrm{g} & \mathrm{Ca}, \mu \mathrm{g} / \mathrm{g} & \mathrm{Fe}, \mu \mathrm{g} / \mathrm{g} & \mathrm{K}, \mu \mathrm{g} / \mathrm{g} & \mathrm{Mg}, \mu \mathrm{g} / \mathrm{g} & \mathrm{Na}, \mu \mathrm{g} / \mathrm{g} \quad \mathrm{P}, \mu \mathrm{g} / \mathrm{g}\end{array}$

\begin{tabular}{|c|c|c|c|c|c|c|c|c|c|c|}
\hline Site 1, Benbrook 03/08/01 @ 1300 rep. 1 & 3201 & ICP/MS & na & 34,200 & 140,000 & 15,600 & 6,280 & 5,940 & 1,310 & 586 \\
\hline RPD for each compound: & & & & 0.3 & 2.1 & 1.9 & 2.7 & 4.8 & 3.1 & 4.0 \\
\hline \multicolumn{11}{|l|}{ Median RPD of sample $=1.9$} \\
\hline Site 1, Benbrook 03/08/01 @ 1300 & 3201 & ICP/MS & 4.97 & 34,300 & 143,000 & 15,900 & 6,450 & 5,660 & 1,270 & 610 \\
\hline Site 1, Benbrook 03/08/01 @ 1300 rep. 2 & 3201 & ICP/MS & na & 33,500 & 137,000 & 15,300 & 6,180 & 5,920 & 1,300 & 582 \\
\hline RPD for each compound: & & & & 2.4 & 4.3 & 3.8 & 4.3 & 4.5 & 2.3 & 4.7 \\
\hline \multicolumn{11}{|l|}{ Median RPD of sample = 3.8} \\
\hline Site 1, Benbrook 03/08/01 @ 1300 rep. 1 & 3201 & ICP/MS & na & 34,200 & 140,000 & 15,600 & 6,280 & 5,940 & 1,310 & 586 \\
\hline Site 1, Benbrook 03/08/01 @ 1300 rep. 2 & 3201 & $\mathrm{ICP} / \mathrm{MS}$ & na & 33,500 & 137,000 & 15,300 & 6,180 & 5,920 & 1,300 & 582 \\
\hline RPD for each compound: & & & & 2.1 & 2.2 & 1.9 & 1.6 & 0.3 & 0.8 & 0.7 \\
\hline \multicolumn{11}{|l|}{ Median RPD of sample $=2.1$} \\
\hline ECO.1 0-5 & 3202 & ICP/MS & 3.50 & 57,800 & 103,000 & 31,900 & 8,910 & 7,590 & 1,070 & 1,010 \\
\hline ECO. $10-5$ rep. 1 & 3202 & ICP/MS & na & 57,000 & 98,600 & 31,800 & 8,640 & 8,160 & 1,180 & 1,030 \\
\hline RPD for each compound: & & & & 1.4 & 4.4 & 0.3 & 3.1 & 7.2 & 9.8 & 2.0 \\
\hline \multicolumn{11}{|l|}{ Median RPD of sample $=2.9$} \\
\hline ECO.1 0-5 & 3202 & ICP/MS & 3.50 & 57,800 & 103,000 & 31,900 & 8,910 & 7,590 & 1,070 & 1,010 \\
\hline ECO. $10-5$ rep. 2 & 3202 & ICP/MS & na & 55,600 & 96,800 & 31,100 & 8,440 & 7,970 & 1,160 & 1,010 \\
\hline RPD for each compound: & & & & 3.9 & 6.2 & 2.5 & 5.4 & 4.9 & 8.1 & 0.0 \\
\hline \multicolumn{11}{|l|}{ Median RPD of sample $=3.0$} \\
\hline ECO.1 0-5 rep. 1 & 3202 & ICP/MS & na & 57,000 & 98,600 & 31,800 & 8,640 & 8,160 & 1,180 & 1,030 \\
\hline ECO.1 0-5 rep. 2 & 3202 & ICP/MS & na & 55,600 & 96,800 & 31,100 & 8,440 & 7,970 & 1,160 & 1,010 \\
\hline RPD for each compound: & & & & 2.5 & 1.8 & 2.2 & 2.3 & 2.4 & 1.7 & 2.0 \\
\hline \multicolumn{11}{|l|}{ Median RPD of sample $=\mathbf{2 . 0}$} \\
\hline Boggy Creek 05/25/01@ @ 0205 & 3209 & ICP/MS & 2.28 & 51,800 & 166,000 & 20,700 & 12,200 & 8,210 & 1,130 & 800 \\
\hline Boggy Creek 05/25/01 @ 0205 rep. 1 & 3209 & $\mathrm{ICP} / \mathrm{MS}$ & na & 51,500 & 164,000 & 20,600 & 12,200 & 7,610 & 1,190 & 796 \\
\hline RPD for each compound: & & & & 0.6 & 1.2 & 0.5 & 0.0 & 7.6 & 5.2 & 0.5 \\
\hline \multicolumn{11}{|l|}{ Median RPD of sample $=1.8$} \\
\hline Boggy Creek 05/25/01@ @ 0205 & 3209 & ICP/MS & 2.28 & 51,800 & 166,000 & 20,700 & 12,200 & 8,210 & 1,130 & 800 \\
\hline Boggy Creek 05/25/01 @ 0205 rep. 2 & 3209 & ICP/MS & na & 52,200 & 165,000 & 20,600 & 12,200 & 7,630 & 1,180 & 808 \\
\hline RPD for each compound: & & & & 0.8 & 0.6 & 0.5 & 0.0 & 7.3 & 4.3 & 1.0 \\
\hline \multicolumn{11}{|l|}{ Median RPD of sample $=1.3$} \\
\hline Boggy Creek 05/25/01@ @ 0205 rep. 1 & 3209 & ICP/MS & na & 51,500 & 164,000 & 20,600 & 12,200 & 7,610 & 1,190 & 796 \\
\hline Boggy Creek 05/25/01 @ 0205 rep. 2 & 3209 & $\mathrm{ICP} / \mathrm{MS}$ & na & 52,200 & 165,000 & 20,600 & 12,200 & 7,630 & 1,180 & 808 \\
\hline
\end{tabular}

Boggy Creek 05/25/01@ 0205 rep. 2 3209 
RPD for each compound:

Median RPD of sample $=0.9$

Barton Springs Sed. Trap 05/10-05/17/01

3210

ICP/MS

ICP/MS

1.22

$\begin{array}{lllllll}34,100 & 141,000 & 16,300 & 6,460 & 62,000 & 820 & 721\end{array}$

Barton Springs Sed. Trap 05/10-05/17/01 rep. 1

3210

na

$142,000 \quad 16,400$

RPD for each compound:

Median RPD of sample $=1.4$

Barton Springs Sed. Trap 05/10-05/17/01

$\begin{array}{llllllll}1.22 & 34,100 & 141,000 & 16,300 & 6,460 & 62,000 & 820 & 721\end{array}$

Barton Springs Sed. Trap

3210

D for each compound:

$\mathrm{ICP} / \mathrm{MS}$

na 33,30

\begin{tabular}{ccccccc}
$\mathbf{2 . 4}$ & 138,000 & 16,100 & 6,240 & 65,200 & 772 & 700 \\
\hline $\mathbf{2 . 2}$ & $\mathbf{1 . 2}$ & $\mathbf{3 . 5}$ & $\mathbf{5 . 0}$ & $\mathbf{6 . 0}$ & $\mathbf{3 . 0}$
\end{tabular}

Barton Springs Sed. Trap 05/10-05/17/01 rep. 1

3210

Barton Springs Sed. Trap 05/10-05/17/01 rep. 2

ICP/MS

$\begin{array}{llllll}\text { na } & 34,000 & 142,000 & 16,400 & 6,370 & 65,500\end{array}$

PD for each compound

Median RPD of sample $=\mathbf{2 . 4}$

Site 5, Downtown 05/04/01@ 2100

$\begin{array}{llllllllll}3342 & \text { ICP/MS } & \text { isa } & 27,900 & 125,000 & 17,000 & 6,270 & 3,880 & 1,150 & 1,650\end{array}$

Site 5, Downtown 05/04/01 @ 2100 dup.

ICP/MS

7.3

RPD for each compound:

Median RPD of sample $=16.4$

CMO.5 40-50

$3427 \quad$ ICP/MS

ICP/MS

0.98

CMO 5 40-50 rep. 1

3427

$\mathrm{n}$

RPD for each compound:

Median RPD of sample $=\mathbf{2} .0$

CMO.5 40-50

ICP/MS

ICP/MS

$32,900 \quad 144,000$

20,700

6,270
7,150

4,780

$1,390 \quad 1,890$

CMO. 5 40-50 rep. 2

34

RPD for each compound:

Median RPD of sample $=3.0$

CMO.5 40-50 rep. 1

$3427 \quad$ ICP/MS

CMO.5 40-50 rep. 2

RPD for each compound:

ICP/MS

$\begin{array}{llll}16.4 & 14.1 & 19.6 & 13.1\end{array}$

20.8

$18.9 \quad 13.6$

Median RPD of sample $=2.2$

Site 5, Downtown 08/16/01@ 0846

Site 5, Downtown 08/16/01@ 0846 dup.

3711

ICP/MS

$3711 \quad$ ICP/MS

na

39,600

$\begin{array}{lllllll}8,470 & 273,000 & 11,900 & 1,900 & 2,440 & 823 & 436\end{array}$

\begin{tabular}{lllllll}
8,420 & 268,000 & 11,800 & 1,790 & 2,390 & 807 & 437 \\
\hline 0.6 & 1.8 & 0.8 & 6.0 & 2.1 & 2.0 & 0.2
\end{tabular}

RPD for each compound: 
Sample ID

Job No.

Method

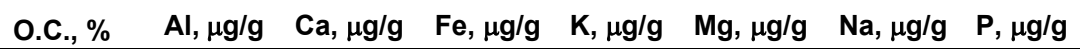
Median RPD of sample $=2.7$

Site 5, Downtown 08/16/01 @ 0846 Dup. $\begin{array}{llllllllll}3711 & \text { ICP/MS } & 7.99 & 39,600 & 150,000 & 26,500 & 7,970 & 5,940 & 1,190 & 1,900\end{array}$ $3711 \quad$ ICP/MS

na 41,000

\begin{tabular}{ccccccc}
41,000 & 151,000 & 26,800 & 7,990 & 6,200 & 1,260 & 1,900 \\
\hline $\mathbf{3 . 5}$ & $\mathbf{0 . 7}$ & $\mathbf{1 . 1}$ & $\mathbf{0 . 3}$ & $\mathbf{4 . 3}$ & $\mathbf{5 . 7}$ & $\mathbf{0 . 0}$
\end{tabular}

RPD for each compound:

Median RPD of sample $=1.8$

Site 5, Downtown 08/16/01 @ 0846 Dup.

$\begin{array}{llllllllll}3711 & \text { ICP/MS } & 7.99 & 39,600 & 150,000 & 26,500 & 7,970 & 5,940 & 1,190 & 1,900\end{array}$

Site 5, Downtown 08/16/01@ 0846 Dup. rep. $2 \quad 3711$ ICP/MS na

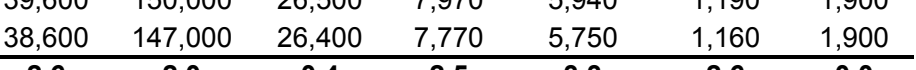

RPD for each compound:

Median RPD of sample $=\mathbf{2} .0$

Site 5, Downtown 08/16/01 @ 0846 Dup. rep. $1 \quad 3711 \quad$ ICP/MS $\quad$ na $\quad 41,000 \quad 151,000 \quad 26,800 \quad 7,990 \quad 6,200 \quad 1,260 \quad 1,900$

\begin{tabular}{|c|c|c|c|c|c|c|c|c|c|c|}
\hline Site 5, Downtown 08/16/01 & 3711 & ICP/MS & na & 38,600 & 147,000 & 26,400 & 7,770 & 5,750 & 1,160 & 1,900 \\
\hline RPD for each compound: & & & & 6.0 & 2.7 & 1.5 & 2.8 & 7.5 & 8.3 & 0.0 \\
\hline
\end{tabular}

Median RPD of sample $=3.8$

Sycamore Creek \#1 08/30/01 @ 0829

$\begin{array}{llllllllll}3744 & \text { ICP/MS } & 4.13 & 62,300 & 139,000 & 32,800 & 12,500 & 7,790 & 1,420 & 974\end{array}$

Sycamore Creek \#1 08/30/01 @ 0829 dup.

3744

ICP/MS

na

\begin{tabular}{ccccccc}
55,600 & 125,000 & 29,500 & 11,300 & 6,620 & 1,280 & 885 \\
\hline $\mathbf{1 1 . 4}$ & $\mathbf{1 0 . 6}$ & $\mathbf{1 0 . 6}$ & $\mathbf{1 0 . 1}$ & $\mathbf{1 6 . 2}$ & $\mathbf{1 0 . 4}$ & $\mathbf{9 . 6}$
\end{tabular}

Median RPD of sample $=11$.

Old Mill Springs 11/16/01 @ 1655

Old Mill Springs 11/16/01@ 1655 dup.

RPD for each compound:

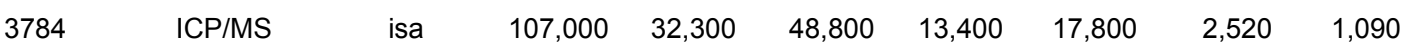

Median RPD of sample $=1.0$

Lake Como Inflow 01/23/02@ 1750

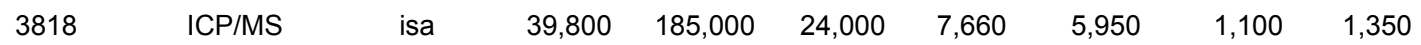

Lake Como Inflow 01/23/02 @ 1750 dup.

$3818 \quad$ ICP/MS

na $\quad 39,200 \quad 183,000$

$23,700 \quad 7,560$

\begin{tabular}{rrr}
5,890 & 1,080 & 1,370 \\
\hline
\end{tabular}

RPD for each compound:

Median RPD of sample $=1.3$

\begin{tabular}{|c|c|c|c|c|c|c|c|c|c|c|}
\hline Aberjona Composite 09/05/2002 & 4315 & ICP/MS & na & 48,500 & 15,200 & 80,200 & 11,800 & 9,860 & 12,100 & 4,160 \\
\hline Aberjona Composite 09/05/2002 dup. & 4315 & ICP/MS & na & 48,800 & 15,200 & 80,400 & 11,700 & 9,800 & 11,900 & 4,240 \\
\hline \multicolumn{3}{|l|}{$\begin{array}{l}\text { RPD for each compound: } \\
\text { Median RPD of sample }=0.5\end{array}$} & & 0.6 & 0.0 & 0.2 & 0.9 & 0.6 & 1.7 & 1.9 \\
\hline \multicolumn{3}{|c|}{ ICP/MS METHOD } & & & & & & & & \\
\hline $\begin{array}{r}\text { MEDIAN RPD } \\
\text { MEDIAN RPD FOR ALL JOBS }\end{array}$ & $\begin{array}{l}\text { MPOUI } \\
\text { INDS = }\end{array}$ & & & 1.5 & 2.0 & 1.4 & 2.4 & 3.2 & 2.2 & 1.5 \\
\hline
\end{tabular}




\begin{tabular}{|c|c|c|c|c|c|c|c|c|c|c|c|c|c|}
\hline Sample ID & $\mathrm{Ti}, \mu \mathrm{g} / \mathrm{g}$ & As, $\mu \mathbf{g} / \mathbf{g}$ & $\mathrm{Ba}, \mu \mathrm{g} / \mathrm{g}$ & $\mathrm{Be}, \mu \mathrm{g} / \mathrm{g}$ & $\mathrm{Cd}, \mu \mathrm{g} / \mathrm{g}$ & Co, $\mu \mathrm{g} / \mathbf{g}$ & $\mathrm{Cr}, \mu \mathrm{g} / \mathrm{g}$ & $\mathrm{Cu}, \mu \mathrm{g} / \mathrm{g}$ & $\mathrm{Hg}, \mu \mathrm{g} / \mathrm{g}$ & $\mathbf{L i}, \mu \mathbf{g} / \mathbf{g}$ & $\mathrm{Mn}, \mu \mathbf{g} / \mathbf{g}$ & $\mathrm{Ni}, \mu \mathrm{g} / \mathrm{g}$ & $\mathrm{Pb}, \mu \mathrm{g} / \mathrm{g}$ \\
\hline MYS.B2 3-4 & $4,4,588$ & 103 & 544 & & 7.9 & 29.5 & 372 & 2404 & 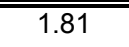 & 43 & 918 & 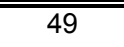 & 617 \\
\hline MYS.B2 3-4 Dup & 4,471 & 102 & 528 & & 7.8 & 27.6 & 376 & 398 & 1.83 & 43 & 921 & 50 & 622 \\
\hline $\begin{array}{l}\text { RPD for each compound: } \\
\text { Median RPD of sample }=1.1\end{array}$ & 2.6 & 0.3 & 3.0 & & 2.2 & 6.7 & 1.1 & 1.5 & 1.1 & 0.1 & 0.4 & 2.0 & 0.8 \\
\hline MYS.B2 12-13 & 4,471 & 140 & 383 & & 8.3 & 29.0 & 496 & 440 & 1.68 & 42 & 863 & 51 & 850 \\
\hline MYS.B2 12-13 Dup & 4,471 & 146 & 402 & & 8.3 & 29.9 & 504 & 444 & 1.76 & 43 & 850 & 53 & 848 \\
\hline $\begin{array}{l}\text { RPD for each compound: } \\
\text { Median RPD of sample }=1.5\end{array}$ & 0.0 & 4.1 & 4.8 & & 0.7 & 3.1 & 1.7 & 0.9 & 4.5 & 1.5 & 1.5 & 3.5 & 0.2 \\
\hline MYS.B2 28-30 & 4,778 & 119 & 365 & & 8.9 & 34.9 & 952 & 396 & 2.18 & 45 & 605 & 47 & 987 \\
\hline MYS.B2 28-30 Dup & 4,667 & 119 & 356 & & 9.1 & 35.3 & 950 & 391 & 2.11 & 44 & 600 & 46 & 997 \\
\hline $\begin{array}{l}\text { RPD for each compound: } \\
\text { Median RPD of sample }=\mathbf{2 . 1}\end{array}$ & 2.4 & 0.1 & 2.5 & & 2.2 & 1.1 & 0.2 & 1.3 & 3.2 & 1.2 & 0.9 & 2.8 & 1.0 \\
\hline HSP.1 18-21 & 3,647 & 13 & 543 & & 1.8 & 6.1 & 72 & 83 & 0.40 & 11 & 486 & 11 & 114 \\
\hline HSP.1 18-21 Dup & 3,647 & 14 & 548 & & 1.9 & 6.5 & 74 & 83 & 0.42 & 12 & 484 & 12 & 114 \\
\hline $\begin{array}{l}\text { RPD for each compound: } \\
\text { Median RPD of sample }=3.5\end{array}$ & 0.0 & 3.8 & 0.9 & & 3.8 & 6.3 & 2.2 & 0.3 & 4.9 & 10.5 & 0.5 & 10.7 & 0.0 \\
\hline HSP.1 30-33 & 3,059 & 0.9 & 569 & & 0.14 & 9.8 & 17 & 8 & 0.12 & 2.1 & 384 & 5.8 & 32 \\
\hline HSP.1 30-33 Dup & 2,941 & 0.8 & 549 & & 0.12 & 8.6 & 14 & 9 & 0.11 & 3.3 & 396 & 5.3 & 37 \\
\hline $\begin{array}{l}\text { RPD for each compound: } \\
\text { Median RPD of sample }=7.0\end{array}$ & 3.9 & 11.8 & 3.6 & & 15.4 & 13.0 & 23.4 & 5.3 & 8.7 & 42.9 & 3.1 & 9.0 & 14.5 \\
\hline HSP.B2 4-5 & 4,125 & 4.8 & 562 & & 0.52 & 14 & 41 & 55 & 0.21 & 21 & 739 & 12 & 55 \\
\hline HSP.B2 4-5 Dup & 3,875 & 4.0 & 542 & & 0.45 & 13 & 40 & 54 & 0.20 & 22 & 711 & 11 & 55 \\
\hline $\begin{array}{l}\text { RPD for each compound: } \\
\text { Median RPD of sample }=4.4\end{array}$ & 6.3 & 18.2 & 3.6 & & 14.4 & 3.7 & 2.1 & 2.9 & 4.9 & 3.0 & 3.9 & 8.1 & 0.0 \\
\hline HSP.B2 8-9 & 4,500 & 8.3 & 523 & & 0.18 & 14 & 50 & 62 & 0.22 & 19 & 692 & 22 & 58 \\
\hline HSP.B2 8-9 Dup & 4,000 & 9.0 & 516 & & 0.16 & 13 & 51 & 61 & 0.21 & 18 & 686 & 20 & 57 \\
\hline $\begin{array}{l}\text { RPD for each compound: } \\
\text { Median RPD of sample }=8.1\end{array}$ & 11.8 & 8.1 & 1.3 & & 11.8 & 10.4 & 3.2 & 1.8 & 4.7 & 9.2 & 0.9 & 9.6 & 1.7 \\
\hline HSP.B3 6-9 & 4,000 & 7.2 & 556 & & 0.39 & 11 & 34 & 47 & 0.16 & 16 & 549 & 14 & 62 \\
\hline HSP.B3 6-9 Dup & 3,882 & 6.8 & 544 & & 0.40 & 11 & 38 & 46 & 0.18 & 15 & 539 & 18 & 65 \\
\hline RPD for each compound: & 3.0 & 5.7 & 2.2 & & 2.5 & 4.6 & 11.8 & 0.9 & 11.8 & 1.7 & 1.8 & 25.2 & 4.7 \\
\hline
\end{tabular}

Median RPD of sample $=\mathbf{3 . 2}$ 


\begin{tabular}{|c|c|c|c|c|c|c|c|c|c|c|c|c|c|}
\hline HSP.B3 21-24 & 4,118 & 3.5 & 584 & & 0.32 & 9.3 & 41 & 52 & 0.17 & 17 & 499 & 15 & 62 \\
\hline HSP.B3 21-24 Dup & 4,235 & 3.2 & 599 & & 0.33 & 10 & 44 & 54 & 0.17 & 16 & 516 & 15 & 61 \\
\hline $\begin{array}{l}\text { RPD for each compound: } \\
\text { Median RPD of sample }=3.4\end{array}$ & 2.8 & 9.0 & 2.5 & & 3.1 & 7.3 & 7.4 & 4.0 & 0.0 & 1.2 & 3.4 & 1.3 & 1.6 \\
\hline & 2.8 & 5.7 & 2.5 & & 3.1 & 6.3 & 2.2 & 1.5 & 4.7 & 1.7 & 1.5 & 8.1 & 1.0 \\
\hline FOS.4 0-5 & 2,990 & 13.0 & 260 & 1.51 & 1.70 & 10.4 & 87.0 & 38.5 & 0.24 & 36.9 & 869 & 26.3 & 108 \\
\hline FOS.4 0-5 rep. 1 & 2,500 & 12.2 & 248 & 1.75 & 1.72 & 9.60 & 70.2 & 35.9 & na & 36.8 & 823 & 23.8 & 108 \\
\hline $\begin{array}{l}\text { RPD for each compound: } \\
\text { Median RPD of sample }=6.5\end{array}$ & 17.9 & 6.3 & 4.7 & 14.7 & 1.2 & 8.0 & 21.4 & 7.0 & & 0.3 & 5.4 & 10.0 & 0.0 \\
\hline FOS.4 0-5 & 2,990 & 13.0 & 260 & 1.51 & 1.70 & 10.4 & 87.0 & 38.5 & 0.24 & 36.9 & 869 & 26.3 & 108 \\
\hline FOS.4 0-5 rep. 2 & 2,900 & 12.1 & 246 & 1.70 & 1.68 & 9.30 & 71.2 & 35.2 & na & 37.0 & 799 & 23.2 & 111 \\
\hline $\begin{array}{l}\text { RPD for each compound: } \\
\text { Median RPD of sample }=8.4\end{array}$ & 3.1 & 7.2 & 5.5 & 11.8 & 1.2 & 11.2 & 20.0 & 9.0 & & 0.3 & 8.4 & 12.5 & 2.7 \\
\hline FOS.4 0-5 rep. 1 & 2,500 & 12.2 & 248 & 1.75 & 1.72 & 9.60 & 70.2 & 35.9 & na & 36.8 & 823 & 23.8 & 108 \\
\hline FOS.4 0-5 rep. 2 & 2,900 & 12.1 & 246 & 1.70 & 1.68 & 9.30 & 71.2 & 35.2 & na & 37.0 & 799 & 23.2 & 111 \\
\hline $\begin{array}{l}\text { RPD for each compound: } \\
\text { Median RPD of sample }=2.0\end{array}$ & 14.8 & 0.8 & 0.8 & 2.9 & 2.4 & 3.2 & 1.4 & 2.0 & & 0.5 & 3.0 & 2.6 & 2.7 \\
\hline FOS.4 55-60 & 3,060 & 220 & 253 & 2.16 & 0.902 & 10.6 & 66.4 & 21.8 & 0.16 & 48.3 & 572 & 25.9 & 243 \\
\hline FOS. 4 55-60 rep. 1 & 2,990 & 223 & 257 & 2.11 & 0.905 & 10.6 & 65.0 & 21.4 & na & 48.6 & 576 & 25.6 & 244 \\
\hline $\begin{array}{l}\text { RPD for each compound: } \\
\text { Median RPD of sample }=1.0\end{array}$ & 2.3 & 1.4 & 1.6 & 2.3 & 0.3 & 0.0 & 2.1 & 1.9 & & 0.6 & 0.7 & 1.2 & 0.4 \\
\hline FOS.4 55-60 & 3,060 & 220 & 253 & 2.16 & 0.902 & 10.6 & 66.4 & 21.8 & 0.16 & 48.3 & 572 & 25.9 & 243 \\
\hline FOS.4 55-60 rep. 2 & 3,010 & 218 & 255 & 2.07 & 0.932 & 10.6 & 65.4 & 21.1 & na & 48.5 & 571 & 25.4 & 240 \\
\hline $\begin{array}{l}\text { RPD for each compound: } \\
\text { Median RPD of sample }=0.9\end{array}$ & 1.6 & 0.9 & 0.8 & 4.3 & 3.3 & 0.0 & 1.5 & 3.3 & & 0.4 & 0.2 & 1.9 & 1.2 \\
\hline FOS. 4 55-60 rep. 1 & 2,990 & 223 & 257 & 2.11 & 0.905 & 10.6 & 65.0 & 21.4 & na & 48.6 & 576 & 25.6 & 244 \\
\hline FOS.4 55-60 rep. 2 & 3,010 & 218 & 255 & 2.07 & 0.932 & 10.6 & 65.4 & 21.1 & na & 48.5 & 571 & 25.4 & 240 \\
\hline $\begin{array}{l}\text { RPD for each compound: } \\
\text { Median RPD of sample }=0.8\end{array}$ & 0.7 & 2.3 & 0.8 & 1.9 & 2.9 & 0.0 & 0.6 & 1.4 & & 0.2 & 0.9 & 0.8 & 1.7 \\
\hline Site 1, Benbrook 03/08/01@ 1300 & 1,950 & 9.30 & 230 & 1.50 & 0.333 & 6.57 & 39.4 & 13.0 & 0.03 & 23.7 & 566 & 18.0 & 15.3 \\
\hline
\end{tabular}


Sample ID

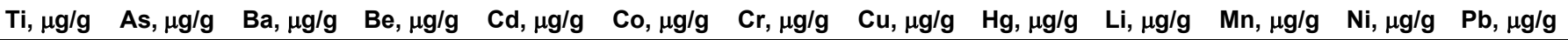

\begin{tabular}{|c|c|c|c|c|c|c|c|c|c|c|c|c|c|}
\hline Site 1, Benbrook 03/08/01 @ $1300 \mathrm{r} \epsilon$ & 2,060 & 8.93 & 232 & 1.50 & 0.365 & 6.39 & 39.8 & 12.8 & na & 24.8 & 556 & 17.3 & 15.1 \\
\hline $\begin{array}{l}\text { RPD for each compound: } \\
\text { Median RPD of sample }=1.9\end{array}$ & 5.5 & 4.1 & 0.9 & 0.0 & 9.2 & 2.8 & 1.0 & 1.6 & & 4.5 & 1.8 & 4.0 & 1.3 \\
\hline Site 1, Benbrook 03/08/01 @ 1300 & 1,950 & 9.30 & 230 & 1.50 & 0.333 & 6.57 & 39.4 & 13.0 & 0.03 & 23.7 & 566 & 18.0 & 15.3 \\
\hline Site 1, Benbrook 03/08/01@ $1300 \mathrm{r} \epsilon$ & 1,930 & 8.92 & 232 & 1.38 & 0.349 & 6.23 & 38.6 & 12.5 & na & 24.2 & 544 & 16.6 & 15.0 \\
\hline $\begin{array}{l}\text { RPD for each compound: } \\
\text { Median RPD of sample }=3.8\end{array}$ & 1.0 & 4.2 & 0.9 & 8.3 & 4.7 & 5.3 & 2.1 & 3.9 & & 2.1 & 4.0 & 8.1 & 2.0 \\
\hline Site 1, Benbrook 03/08/01@ 1300 rє & 2,060 & 8.93 & 232 & 1.50 & 0.365 & 6.39 & 39.8 & 12.8 & na & 24.8 & 556 & 17.3 & 15.1 \\
\hline Site 1, Benbrook 03/08/01@ $1300 \mathrm{r} \epsilon$ & 1,930 & 8.92 & 232 & 1.38 & 0.349 & 6.23 & 38.6 & 12.5 & na & 24.2 & 544 & 16.6 & 15.0 \\
\hline $\begin{array}{l}\text { RPD for each compound: } \\
\text { Median RPD of sample }=2.1\end{array}$ & 6.5 & 0.1 & 0.0 & 8.3 & 4.5 & 2.5 & 3.1 & 2.4 & & 2.4 & 2.2 & 4.1 & 0.7 \\
\hline ECO.1 0-5 & 3,220 & 15.9 & 413 & 2.42 & 3.18 & 10.6 & 72.1 & 39.9 & 0.11 & 42.5 & 731 & 30.7 & 124 \\
\hline ECO.1 0-5 rep. 1 & 3,380 & 16.5 & 434 & 2.24 & 3.30 & 10.3 & 71.7 & 39.8 & na & 43.1 & 734 & 29.6 & 126 \\
\hline $\begin{array}{l}\text { RPD for each compound: } \\
\text { Median RPD of sample }=\mathbf{2 . 9}\end{array}$ & 4.8 & 3.7 & 5.0 & 7.7 & 3.7 & 2.9 & 0.6 & 0.3 & & 1.4 & 0.4 & 3.6 & 1.6 \\
\hline ECO.1 0-5 & 3,220 & 15.9 & 413 & 2.42 & 3.18 & 10.6 & 72.1 & 39.9 & 0.11 & 42.5 & 731 & 30.7 & 124 \\
\hline ECO. $10-5$ rep. 2 & 3,220 & 16.1 & 422 & 2.20 & 3.27 & 10.1 & 70.5 & 38.6 & na & 42.3 & 720 & 28.6 & 124 \\
\hline $\begin{array}{l}\text { RPD for each compound: } \\
\text { Median RPD of sample }=3.0\end{array}$ & 0.0 & 1.3 & 2.2 & 9.5 & 2.8 & 4.8 & 2.2 & 3.3 & & 0.5 & 1.5 & 7.1 & 0.0 \\
\hline ECO. $10-5$ rep. 1 & 3,380 & 16.5 & 434 & 2.24 & 3.30 & 10.3 & 71.7 & 39.8 & na & 43.1 & 734 & 29.6 & 126 \\
\hline ECO. $10-5$ rep. 2 & 3,220 & 16.1 & 422 & 2.20 & 3.27 & 10.1 & 70.5 & 38.6 & na & 42.3 & 720 & 28.6 & 124 \\
\hline $\begin{array}{l}\text { RPD for each compound: } \\
\text { Median RPD of sample }=2.0\end{array}$ & 4.8 & 2.5 & 2.8 & 1.8 & 0.9 & 2.0 & 1.7 & 3.1 & & 1.9 & 1.9 & 3.4 & 1.6 \\
\hline Boggy Creek 05/25/01 @ 0205 & 3,070 & 11.4 & 239 & 1.21 & 0.526 & 8.43 & 54.8 & 20.5 & 0.04 & 28.9 & 540 & 22.7 & 35.5 \\
\hline Boggy Creek 05/25/01 @ 0205 rep. 1 & 2,860 & 11.2 & 244 & 1.26 & 0.548 & 8.20 & 54.7 & 19.6 & na & 29.8 & 541 & 22.3 & 35.4 \\
\hline $\begin{array}{l}\text { RPD for each compound: } \\
\text { Median RPD of sample }=1.8\end{array}$ & 7.1 & 1.8 & 2.1 & 4.0 & 4.1 & 2.8 & 0.2 & 4.5 & & 3.1 & 0.2 & 1.8 & 0.3 \\
\hline Boggy Creek 05/25/01 @ 0205 & 3,070 & 11.4 & 239 & 1.21 & 0.526 & 8.43 & 54.8 & 20.5 & 0.04 & 28.9 & 540 & 22.7 & 35.5 \\
\hline Boggy Creek 05/25/01 @ 0205 rep. \& & 3,110 & 11.5 & 246 & 1.30 & 0.549 & 8.10 & 56.3 & 20.5 & na & 29.2 & 546 & 22.1 & 35.4 \\
\hline $\begin{array}{l}\text { RPD for each compound: } \\
\text { Median RPD of sample }=1.3\end{array}$ & 1.3 & 0.9 & 2.9 & 7.2 & 4.3 & 4.0 & 2.7 & 0.0 & & 1.0 & 1.1 & 2.7 & 0.3 \\
\hline $\begin{array}{l}\text { Boggy Creek 05/25/01 @ } 0205 \text { rep. } 1 \\
\text { Boggy Creek 05/25/01@ @ } 0205 \text { rep. } 2\end{array}$ & $\begin{array}{l}2,860 \\
3,110 \\
\end{array}$ & $\begin{array}{l}11.2 \\
11.5\end{array}$ & $\begin{array}{l}244 \\
246 \\
\end{array}$ & $\begin{array}{l}1.26 \\
1.30 \\
\end{array}$ & $\begin{array}{l}0.548 \\
0.549 \\
\end{array}$ & $\begin{array}{l}8.20 \\
8.10\end{array}$ & $\begin{array}{l}54.7 \\
56.3\end{array}$ & $\begin{array}{l}19.6 \\
20.5 \\
\end{array}$ & $\begin{array}{l}\text { na } \\
\text { na }\end{array}$ & $\begin{array}{l}29.8 \\
29.2 \\
\end{array}$ & $\begin{array}{l}541 \\
546 \\
\end{array}$ & $\begin{array}{l}22.3 \\
22.1\end{array}$ & $\begin{array}{l}35.4 \\
35.4 \\
\end{array}$ \\
\hline
\end{tabular}


Sample ID

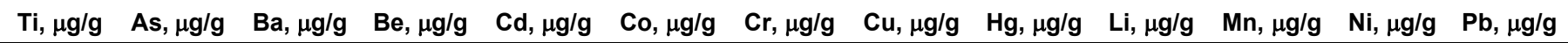
RPD for each compound:

Median RPD of sample $=0.9$

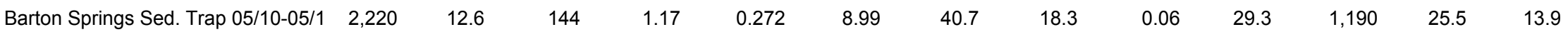

\begin{tabular}{|c|c|c|c|c|c|c|c|c|c|c|c|c|c|}
\hline Barton Springs Sed. Trap 05/10-05/1 & 2,580 & 12.9 & 146 & 1.20 & 0.296 & 8.85 & 43.0 & 18.4 & na & 29.4 & 1,200 & 25.2 & 14.1 \\
\hline RPD for each compound: & 15.0 & $\mathbf{2 . 4}$ & 1.4 & 2.5 & 8.5 & 1.6 & 5.5 & 0.5 & & 0.3 & $\begin{array}{llll}0.8 & & \\
\end{array}$ & 1.2 & 1.4 \\
\hline
\end{tabular}

Median RPD of sample $=1$.

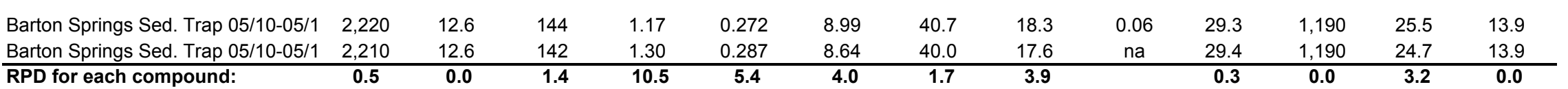

Median RPD of sample $=\mathbf{2} .2$

\begin{tabular}{|c|c|c|c|c|c|c|c|c|c|c|c|c|c|}
\hline Barton Springs Sed. Trap 05/10-05/1 & 2,580 & 12.9 & 146 & 1.20 & 0.296 & 8.85 & 43.0 & 18.4 & na & 29.4 & 1,200 & 25.2 & 14.1 \\
\hline Barton Springs Sed. Trap 05/10-05/1 & 2,210 & 12.6 & 142 & 1.30 & 0.287 & 8.64 & 40.0 & 17.6 & na & 29.4 & 1,190 & 24.7 & 13.9 \\
\hline PD for each compound: & 15.4 & 2.4 & 2.8 & 8.0 & 3.1 & 2.4 & 7.2 & 4.4 & & 0.0 & 0.8 & 2.0 & 1.4 \\
\hline
\end{tabular}

Median RPD of sample $=\mathbf{2 . 4}$

\begin{tabular}{|c|c|c|c|c|c|c|c|c|c|c|c|c|c|}
\hline Site 5, Downtown 05/04/01 @ 2100 & 1,890 & 7.01 & 240 & 0.911 & 1.33 & 6.12 & 46.6 & 61.3 & isa & 16.6 & 324 & 21.1 & 114 \\
\hline Site 5, Downtown 05/04/01 @ 2100 c & 1,830 & 8.18 & 279 & 1.17 & 1.68 & 7.41 & 55.1 & 72.1 & 0.22 & 19.7 & 386 & 26.5 & 134 \\
\hline RPD for each compound: & 3.2 & 15.4 & 15.0 & 24.9 & 23.3 & 19.1 & 16.7 & 16.2 & & 17.1 & 17.5 & 22.7 & 16.1 \\
\hline \multicolumn{14}{|l|}{ Median RPD of sample $=16.4$} \\
\hline CMO.5 40-50 & 472 & 5.80 & 97.7 & 0.401 & 0.455 & 3.75 & 28.7 & 9.27 & 0.05 & 5.67 & 367 & 18.0 & 112 \\
\hline CMO.5 40-50 rep. 1 & 484 & 6.61 & 99.7 & 0.456 & 0.472 & 3.66 & 27.0 & 9.08 & na & 5.50 & 362 & 17.9 & 108 \\
\hline $\begin{array}{l}\text { RPD for each compound: } \\
\text { Median RPD of sample }=\mathbf{2 . 0}\end{array}$ & 2.5 & 13.1 & 2.0 & 12.8 & 3.7 & 2.4 & 6.1 & 2.1 & & 3.0 & 1.4 & 0.6 & 3.6 \\
\hline CMO.5 40-50 & 472 & 5.80 & 97.7 & 0.401 & 0.455 & 3.75 & 28.7 & 9.27 & 0.05 & 5.67 & 367 & 18.0 & 112 \\
\hline CMO.5 40-50 rep. 2 & 460 & 6.50 & 102 & 0.394 & 0.466 & 3.62 & 27.8 & 9.00 & na & 5.38 & 354 & 17.9 & 111 \\
\hline $\begin{array}{l}\text { RPD for each compound: } \\
\text { Median RPD of sample }=3.0\end{array}$ & 2.6 & 11.4 & 4.3 & 1.8 & 2.4 & 3.5 & 3.2 & 3.0 & & 5.2 & 3.6 & 0.6 & 0.9 \\
\hline CMO.5 40-50 rep. 1 & 484 & 6.61 & 99.7 & 0.456 & 0.472 & 3.66 & 27.0 & 9.08 & na & 5.50 & 362 & 17.9 & 108 \\
\hline CMO.5 40-50 rep. 2 & 460 & 6.50 & 102 & 0.394 & 0.466 & 3.62 & 27.8 & 9.00 & na & 5.38 & 354 & 17.9 & 111 \\
\hline $\begin{array}{l}\text { RPD for each compound: } \\
\text { Median RPD of sample }=2.2\end{array}$ & 5.1 & 1.7 & 2.3 & 14.6 & 1.3 & 1.1 & 2.9 & 0.9 & & 2.2 & 2.2 & 0.0 & 2.7 \\
\hline Site 5, Downtown 08/16/01 @ 0846 & 2,390 & 11.7 & 383 & 1.52 & 2.73 & 11.4 & 77.0 & 281 & 0.31 & 27.3 & 588 & 32.2 & 237 \\
\hline Site 5, Downtown 08/16/01 @ 0846 c & 2,480 & 11.5 & 370 & 1.49 & 2.52 & 11.0 & 72.4 & 257 & 0.32 & 26.7 & 585 & 31.9 & 236 \\
\hline RPD for each compound: & 3.7 & 1.7 & 3.5 & 2.0 & $\mathbf{8 . 0}$ & 3.6 & 6.2 & 8.9 & 3.2 & 2.2 & 0.5 & 0.9 & 0.4 \\
\hline
\end{tabular}


Sample ID

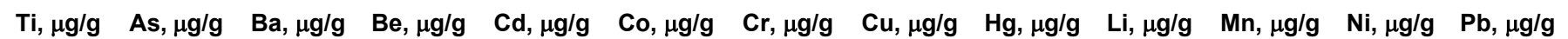
Median RPD of sample $=2.7$

Site 5, Downtown 08/16/01 @ 0846 [ 2,480

Site 5, Downtown 08/16/01 @ 0846 [ 2,520

\begin{tabular}{cccccccccccc}
11.5 & 370 & 1.49 & 2.52 & 11.0 & 72.4 & 257 & 0.32 & 26.7 & 585 & 31.9 & 236 \\
12.2 & 380 & 1.51 & 2.59 & 11.2 & 71.9 & 265 & na & 27.0 & 595 & 32.0 & 249 \\
\hline $\mathbf{5 . 9}$ & $\mathbf{2 . 7}$ & $\mathbf{1 . 3}$ & $\mathbf{2 . 7}$ & $\mathbf{1 . 8}$ & $\mathbf{0 . 7}$ & $\mathbf{3 . 1}$ & & $\mathbf{1 . 1}$ & $\mathbf{1 . 7}$ & $\mathbf{0 . 3}$ & $\mathbf{5 . 4}$
\end{tabular}

Median RPD of sample $=1.8$

\begin{tabular}{|c|c|c|c|c|c|c|c|c|c|c|c|c|c|}
\hline Downtown 0 & 2,480 & 11.5 & 370 & 1.49 & 2.52 & 11.0 & 72.4 & 257 & 0.32 & 26.7 & 585 & 31.9 & 236 \\
\hline Site 5, Downtown 08/16/01@0846 [ & 2,480 & 11.4 & 370 & 1.41 & 2.47 & 10.9 & 70.2 & 253 & na & 25.9 & 580 & 30.8 & 237 \\
\hline
\end{tabular}

Median RPD of sample $=2.0$

\begin{tabular}{|c|c|c|c|c|c|c|c|c|c|c|c|c|c|}
\hline Site 5, Downtown 08/16/01 @ 0846 [ & 2,520 & 12.2 & 380 & 1.51 & 2.59 & 11.2 & 71.9 & 265 & na & 27.0 & 595 & 32.0 & 249 \\
\hline Site 5, Downtown 08/16/01 @ 0846 [ & 2,480 & 11.4 & 370 & 1.41 & 2.47 & 10.9 & 70.2 & 253 & na & 25.9 & 580 & 30.8 & 237 \\
\hline RPD for each compound: & 1.6 & 6.8 & 2.7 & 6.8 & 4.7 & 2.7 & 2.4 & 4.6 & & 4.2 & 2.6 & 3.8 & 4.9 \\
\hline
\end{tabular}

Median RPD of sample $=\mathbf{3 . 8}$

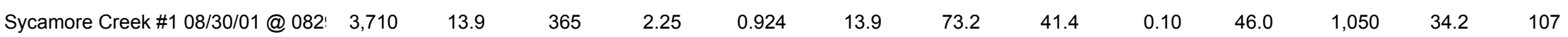

$\begin{array}{llllllllllllll}\text { Sycamore Creek \#1 08/30/01 @ 082: } & 3,360 & 12.0 & 324 & 1.96 & 0.811 & 12.1 & 66.4 & 36.6 & \text { na } & 39.1 & 945 & 30.4 & 93.7\end{array}$

RPD for each compound:

Median RPD of sample $=\mathbf{1 1 . 8}$

\begin{tabular}{|c|c|c|c|c|c|c|c|c|c|c|c|c|c|}
\hline Old Mill Springs 11/16/01@1655 & 6,620 & 24.2 & 257 & 3.30 & 0.619 & 17.2 & 94.8 & 45.0 & na & 71.2 & 1380 & 47.1 & 35.3 \\
\hline Old Mill Springs 11/16/01 @ 1655 du & 6,580 & 23.8 & 253 & 3.10 & 0.614 & 17.1 & 91.5 & 44.7 & na & 71.1 & 1360 & 46.2 & 34.9 \\
\hline RPD for each compound: & 0.6 & 1.7 & 1.6 & 6.2 & 0.8 & 0.6 & 3.5 & 0.7 & & 0.1 & 1.5 & 1.9 & 1.1 \\
\hline
\end{tabular}

RPD for each compound:

Median RPD of sample $=1.0$

\begin{tabular}{|c|c|c|c|c|c|c|c|c|c|c|c|c|c|}
\hline Lake Como Inflow 01/23/02 @ 1750 & 1,920 & 10.4 & 300 & 1.48 & 1.46 & 8.95 & 56.7 & 63.6 & 0.13 & 23.3 & 820 & 29.4 & 121 \\
\hline Lake Como Inflow 01/23/02 @ 1750 । & 1,960 & 10.6 & 296 & 1.54 & 1.42 & 8.88 & 55.7 & 64.1 & na & 22.7 & 816 & 28.9 & 121 \\
\hline RPD for each compound: & 2.1 & 1.9 & 1.3 & 4.0 & 2.8 & 0.8 & 1.8 & 0.8 & & 2.6 & 0.5 & 1.7 & 0.0 \\
\hline
\end{tabular}

Median RPD of sample $=1.3$

\begin{tabular}{|c|c|c|c|c|c|c|c|c|c|c|c|c|c|}
\hline Aberjona Composite 09/05/2002 & 3,780 & 270 & 449 & 2.74 & 7.79 & 35.1 & 420 & 379 & & 37.0 & 11,600 & 51.2 & 585 \\
\hline Aberjona Composite 09/05/2002 dup & 3,790 & 271 & 447 & 2.64 & 7.76 & 35.1 & 418 & 379 & & 36.9 & 11,700 & 51.6 & 590 \\
\hline $\begin{array}{l}\text { RPD for each compound: } \\
\text { Median RPD of sample }=0.5\end{array}$ & 0.3 & 0.4 & 0.4 & 3.7 & 0.4 & 0.0 & 0.5 & 0.0 & & 0.3 & 0.9 & 0.8 & 0.9 \\
\hline & 2.8 & 2.3 & 2.0 & 5.9 & 3.0 & 2.6 & 2.5 & 3.0 & 3.2 & 1.6 & 1.4 & 2.3 & 1.3 \\
\hline
\end{tabular}




\begin{tabular}{|c|c|c|c|c|}
\hline Sample ID & Sc, $\mu \mathbf{g} / \mathbf{g}$ & $\mathrm{Sr}, \mu \mathbf{g} / \mathbf{g}$ & $\mathbf{V}, \mu \mathbf{g} / \mathbf{g}$ & $\mathrm{Zn}, \mu \mathrm{g} / \mathrm{g}$ \\
\hline MYS.B2 3-4 & 17.3 & 156 & 107 & 1960 \\
\hline MYS.B2 3-4 Dup & 19.0 & 146 & 106 & 1960 \\
\hline RPD for each compound: & 9.2 & 6.6 & 0.9 & 0.0 \\
\hline \multicolumn{5}{|l|}{ Median RPD of sample $=1.1$} \\
\hline MYS.B2 12-13 & 20.4 & 134 & 115 & 2410 \\
\hline MYS.B2 12-13 Dup & 20.4 & 145 & 116 & 2430 \\
\hline RPD for each compound: & 0.0 & 8.1 & 0.9 & 0.8 \\
\hline \multicolumn{5}{|l|}{ Median RPD of sample $=1.5$} \\
\hline MYS.B2 28-30 & 18.6 & 159 & 154 & 3190 \\
\hline MYS.B2 28-30 Dup & 17.2 & 148 & 151 & 3170 \\
\hline RPD for each compound: & 7.8 & 7.0 & 2.0 & 0.6 \\
\hline \multicolumn{5}{|l|}{ Median RPD of sample $=2.1$} \\
\hline HSP.1 18-21 & 12 & 161 & 59 & 132 \\
\hline HSP.1 18-21 Dup & 12 & 166 & 63 & 128 \\
\hline RPD for each compound: & 3.3 & 2.9 & 7.3 & 2.7 \\
\hline \multicolumn{5}{|l|}{ Median RPD of sample $=3.5$} \\
\hline HSP.1 30-33 & 6.4 & 125 & 34 & 30 \\
\hline HSP.1 30-33 Dup & 5.6 & 129 & 34 & 29 \\
\hline RPD for each compound: & 13.3 & 3.0 & 0.0 & 2.6 \\
\hline \multicolumn{5}{|l|}{ Median RPD of sample $=7.0$} \\
\hline HSP.B2 4-5 & 19 & 159 & 60 & 201 \\
\hline HSP.B2 4-5 Dup & 19 & 151 & 56 & 201 \\
\hline RPD for each compound: & 1.7 & 5.0 & 6.5 & 0.0 \\
\hline \multicolumn{5}{|l|}{ Median RPD of sample $=4.4$} \\
\hline HSP.B2 8-9 & 17 & 153 & 68 & 218 \\
\hline HSP.B2 8-9 Dup & 18 & 148 & 60 & 199 \\
\hline RPD for each compound: & 8.1 & 3.3 & 11.8 & 9.1 \\
\hline \multicolumn{5}{|l|}{ Median RPD of sample $=8.1$} \\
\hline HSP.B3 6-9 & 11 & 150 & 63 & 191 \\
\hline HSP.B3 6-9 Dup & 10 & 145 & 57 & 201 \\
\hline RPD for each compound: & 3.8 & 3.4 & 8.9 & 5.1 \\
\hline
\end{tabular}




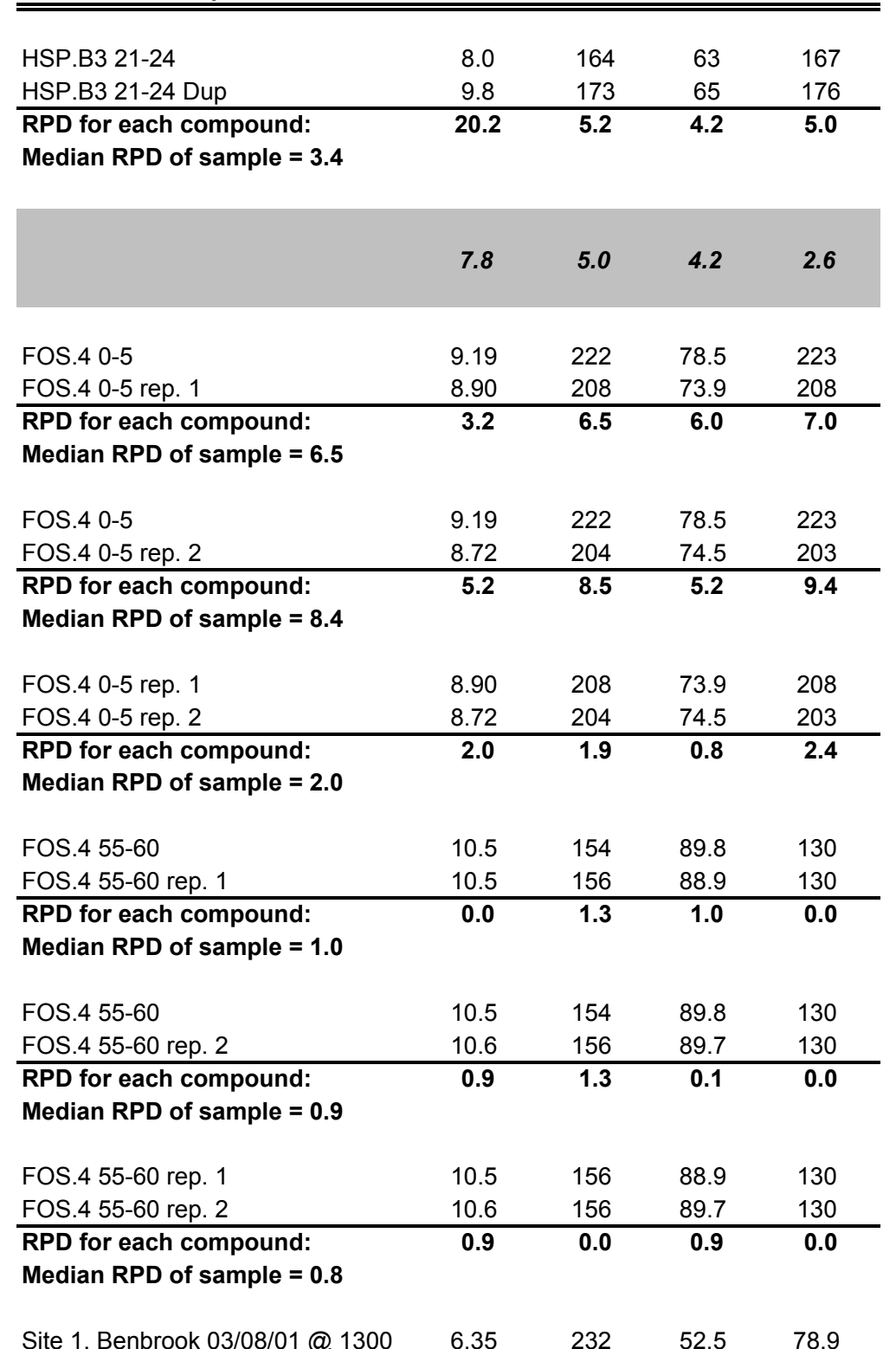




\begin{tabular}{|c|c|c|c|c|}
\hline Sample ID & Sc, $\mu \mathrm{g} / \mathrm{g}$ & $\mathrm{Sr}, \mu \mathrm{g} / \mathrm{g}$ & $\mathbf{V}, \mu \mathbf{g} / \mathbf{g}$ & $Z \mathbf{n}, \mu \mathbf{g} / \mathbf{g}$ \\
\hline Site 1, Benbrook 03/08/01 @ $1300 \mathrm{r} \epsilon$ & 6.27 & 228 & 52.9 & 78.5 \\
\hline $\begin{array}{l}\text { RPD for each compound: } \\
\text { Median RPD of sample }=1.9\end{array}$ & 1.3 & 1.7 & 0.8 & 0.5 \\
\hline Site 1, Benbrook 03/08/01 @ 1300 & 6.35 & 232 & 52.5 & 78.9 \\
\hline Site 1, Benbrook 03/08/01@ $1300 \mathrm{r} \epsilon$ & 6.19 & 224 & 52.1 & 76.4 \\
\hline $\begin{array}{l}\text { RPD for each compound: } \\
\text { Median RPD of sample }=3.8\end{array}$ & 2.6 & 3.5 & 0.8 & 3.2 \\
\hline Site 1, Benbrook 03/08/01@ 1300 r & 6.27 & 228 & 52.9 & 78.5 \\
\hline Site 1, Benbrook 03/08/01@ $1300 \mathrm{r} \epsilon$ & 6.19 & 224 & 52.1 & 76.4 \\
\hline $\begin{array}{l}\text { RPD for each compound: } \\
\text { Median RPD of sample }=\mathbf{2 . 1}\end{array}$ & 1.3 & 1.8 & 1.5 & 2.7 \\
\hline ECO.1 0-5 & 9.86 & 238 & 87.6 & 297 \\
\hline ECO.1 0-5 rep. 1 & 9.66 & 237 & 90.2 & 292 \\
\hline $\begin{array}{l}\text { RPD for each compound: } \\
\text { Median RPD of sample }=2.9\end{array}$ & 2.0 & 0.4 & 2.9 & 1.7 \\
\hline ECO.1 0-5 & 9.86 & 238 & 87.6 & 297 \\
\hline ECO. 1 0-5 rep. 2 & 9.47 & 231 & 88.0 & 287 \\
\hline $\begin{array}{l}\text { RPD for each compound: } \\
\text { Median RPD of sample }=3.0\end{array}$ & 4.0 & 3.0 & 0.5 & 3.4 \\
\hline ECO.1 0-5 rep. 1 & 9.66 & 237 & 90.2 & 292 \\
\hline ECO.1 0-5 rep. 2 & 9.47 & 231 & 88.0 & 287 \\
\hline $\begin{array}{l}\text { RPD for each compound: } \\
\text { Median RPD of sample }=\mathbf{2 . 0}\end{array}$ & 2.0 & 2.6 & 2.5 & 1.7 \\
\hline Boggy Creek 05/25/01 @ 0205 & 9.08 & 390 & 87.3 & 136 \\
\hline Boggy Creek 05/25/01 @ 0205 rep. 1 & 8.80 & 381 & 88.5 & 137 \\
\hline $\begin{array}{l}\text { RPD for each compound: } \\
\text { Median RPD of sample }=1.8\end{array}$ & 3.1 & 2.3 & 1.4 & 0.7 \\
\hline Boggy Creek 05/25/01 @ 0205 & 9.08 & 390 & 87.3 & 136 \\
\hline Boggy Creek 05/25/01 @ 0205 rep. z & 8.96 & 381 & 89.3 & 136 \\
\hline $\begin{array}{l}\text { RPD for each compound: } \\
\text { Median RPD of sample }=1.3\end{array}$ & 1.3 & 2.3 & 2.3 & 0.0 \\
\hline Boggy Creek 05/25/01 @ 0205 rep. 1 & 8.80 & 381 & 88.5 & 137 \\
\hline Boggy Creek 05/25/01 @ 0205 rep. z & 8.96 & 381 & 89.3 & 136 \\
\hline
\end{tabular}




\begin{tabular}{|c|c|c|c|c|}
\hline Sample ID & Sc, $\mu \mathrm{g} / \mathrm{g}$ & $\mathrm{Sr}, \mu \mathrm{g} / \mathrm{g}$ & $\mathbf{V}, \mu \mathbf{g} / \mathbf{g}$ & $\mathrm{Zn}, \mu \mathrm{g} / \mathbf{g}$ \\
\hline $\begin{array}{l}\text { RPD for each compound: } \\
\text { Median RPD of sample }=0.9\end{array}$ & 1.8 & 0.0 & 0.9 & 0.7 \\
\hline Barton Springs Sed. Trap 05/10-05/1 & 6.30 & 105 & 70.0 & 53.2 \\
\hline Barton Springs Sed. Trap 05/10-05/1 & 6.50 & 104 & 71.0 & 54.3 \\
\hline $\begin{array}{l}\text { RPD for each compound: } \\
\text { Median RPD of sample }=1.4\end{array}$ & 3.1 & 1.0 & 1.4 & 2.0 \\
\hline Barton Springs Sed. Trap 05/10-05/1 & 6.30 & 105 & 70.0 & 53.2 \\
\hline Barton Springs Sed. Trap 05/10-05/1 & 6.19 & 101 & 69.6 & 52.8 \\
\hline $\begin{array}{l}\text { RPD for each compound: } \\
\text { Median RPD of sample }=2.2\end{array}$ & 1.8 & 3.9 & 0.6 & 0.8 \\
\hline Barton Springs Sed. Trap 05/10-05/1 & 6.50 & 104 & 71.0 & 54.3 \\
\hline Barton Springs Sed. Trap 05/10-05/1 & 6.19 & 101 & 69.6 & 52.8 \\
\hline $\begin{array}{l}\text { RPD for each compound: } \\
\text { Median RPD of sample }=2.4\end{array}$ & 4.9 & 2.9 & 2.0 & 2.8 \\
\hline Site 5, Downtown 05/04/01 @ 2100 & 4.80 & 252 & 43.6 & 536 \\
\hline Site 5, Downtown 05/04/01@ 2100 c & 5.46 & 296 & 50.8 & 636 \\
\hline $\begin{array}{l}\text { RPD for each compound: } \\
\text { Median RPD of sample }=16.4\end{array}$ & 12.9 & 16.1 & 15.3 & 17.1 \\
\hline CMO.5 40-50 & 2.09 & 379 & 28.0 & 52.4 \\
\hline CMO.5 40-50 rep. 1 & 2.12 & 386 & 28.1 & 53.2 \\
\hline $\begin{array}{l}\text { RPD for each compound: } \\
\text { Median RPD of sample }=2.0\end{array}$ & 1.4 & 1.8 & 0.4 & 1.5 \\
\hline CMO.5 40-50 & 2.09 & 379 & 28.0 & 52.4 \\
\hline CMO.5 40-50 rep. 2 & 2.06 & 379 & 26.9 & 51.3 \\
\hline $\begin{array}{l}\text { RPD for each compound: } \\
\text { Median RPD of sample }=3.0\end{array}$ & 1.4 & 0.0 & 4.0 & 2.1 \\
\hline CMO.5 40-50 rep. 1 & 2.12 & 386 & 28.1 & 53.2 \\
\hline CMO.5 40-50 rep. 2 & 2.06 & 379 & 26.9 & 51.3 \\
\hline $\begin{array}{l}\text { RPD for each compound: } \\
\text { Median RPD of sample }=2.2\end{array}$ & 2.9 & 1.8 & 4.4 & 3.6 \\
\hline Site 5, Downtown 08/16/01 @ 0846 & 7.85 & 335 & 68.6 & 1,600 \\
\hline Site 5, Downtown 08/16/01@ @ 0846 c & 7.49 & 319 & 66.8 & 1,560 \\
\hline RPD for each compound: & 4.7 & 4.9 & 2.7 & 2.5 \\
\hline
\end{tabular}


Sample ID

Sc, $\mu \mathrm{g} / \mathbf{g} \quad \mathrm{Sr}, \mu \mathrm{g} / \mathrm{g} \quad \mathrm{V}, \mu \mathrm{g} / \mathrm{g} \quad \mathrm{Zn}, \mu \mathrm{g} / \mathrm{g}$

Median RPD of sample $=2.7$

Site 5, Downtown 08/16/01 @ 0846 [ $\quad 7.49 \quad 319 \quad 66.8 \quad 1,560$

\begin{tabular}{lccccc} 
Site 5, Downtown 08/16/01 @ 0846 [ & 7.71 & 330 & 67.2 & 1,600 \\
\hline RPD for each compound: & $\mathbf{2 . 9}$ & $\mathbf{3 . 4}$ & $\mathbf{0 . 6}$ & $\mathbf{2 . 5}$
\end{tabular}

Median RPD of sample $=1.8$

Site 5, Downtown 08/16/01 @ 0846 [ $7.49 \quad 319 \quad 66.8 \quad 1,560$

\begin{tabular}{lcccc} 
Site 5, Downtown 08/16/01 @ 0846 [ & 7.48 & 315 & 65.4 & 1,440 \\
\hline RPD for each compound: & $\mathbf{0 . 1}$ & $\mathbf{1 . 3}$ & $\mathbf{2 . 1}$ & $\mathbf{8 . 0}$
\end{tabular}

Median RPD of sample $=\mathbf{2} .0$

Site 5, Downtown 08/16/01 @ $0846\left[\begin{array}{lllll}7 & 7.71 & 330 & 67.2 & 1,600\end{array}\right.$

\begin{tabular}{lccccc} 
Site 5, Downtown 08/16/01 @ 0846 [ & 7.48 & 315 & 65.4 & 1,440 \\
\hline RPD for each compound: & $\mathbf{3 . 0}$ & $\mathbf{4 . 7}$ & $\mathbf{2 . 7}$ & $\mathbf{1 0 . 5}$
\end{tabular}

Median RPD of sample $=3.8$

Sycamore Creek \#1 08/30/01 @ 082 $\quad 11.6 \quad 311 \quad 92.6 \quad 258$

\begin{tabular}{lrrrrr} 
Sycamore Creek \#1 08/30/01 @ 082 & 10.6 & 276 & 84.2 & 223 \\
\hline RPD for each compound: & $\mathbf{9 . 0}$ & $\mathbf{1 1 . 9}$ & $\mathbf{9 . 5}$ & $\mathbf{1 4 . 6}$
\end{tabular}

Median RPD of sample $=\mathbf{1 1 . 8}$

Old Mill Springs 11/16/01 @ 1655

Old Mill Springs 11/16/01 @ 1655 du

RPD for each compound:

\begin{tabular}{rrrr}
16.8 & 201 & 144 & 160 \\
16.8 & 199 & 142 & 158 \\
\hline 0.0 & $\mathbf{1 . 0}$ & $\mathbf{1 . 4}$ & $\mathbf{1 . 3}$
\end{tabular}

Median RPD of sample $=1.0$

Lake Como Inflow 01/23/02 @ $1750 \quad 6.70 \quad 327 \quad 67.9 \quad 421$

\begin{tabular}{lrrrrr} 
Lake Como Inflow 01/23/02 @ 1750, & 6.62 & 326 & 68.4 & 412 \\
\hline RPD for each compound: & $\mathbf{1 . 2}$ & $\mathbf{0 . 3}$ & $\mathbf{0 . 7}$ & $\mathbf{2 . 2}$
\end{tabular}

Median RPD of sample $=1.3$

$\begin{array}{lllll}\text { Aberjona Composite 09/05/2002 } & 9.34 & 138 & 119 & 1,880\end{array}$

\begin{tabular}{lcccc} 
Aberjona Composite 09/05/2002 dup & 9.33 & 138 & 118 & 1,870 \\
\hline RPD for each compound: & $\mathbf{0 . 1}$ & $\mathbf{0 . 0}$ & $\mathbf{0 . 8}$ & $\mathbf{0 . 5}$
\end{tabular}

Median RPD of sample $=0.5$

$\begin{array}{llll}2.0 & 1.9 & 1.4 & 2.1\end{array}$


Table B9. Quality control sample results included with samples analyzed for ${ }^{137} \mathrm{Cs}$.

\begin{tabular}{|l|l|c|c|}
\hline \multicolumn{1}{|c|}{ Sample set } & $\begin{array}{c}\text { RPD of } \\
\text { duplicate sample }\end{array}$ & $\begin{array}{c}\text { Blank } \\
\text { Concentration (pCi/g) }\end{array}$ & $\begin{array}{c}\text { Laboratory Control } \\
\text { Sample Recovery }\end{array}$ \\
\hline Lake Como samples & CMO.1 60-65: 15.3\% & $-0.00274 \pm 0.0321$ & $101.79 \%$ \\
\hline Echo Lake samples & ECO.1 10-15: 28.0\% & $0.0157 \pm 0.0327$ & $98.71 \%$ \\
\hline Fosdic Lake samples & FOS.2 0-10: $64.6 \%$ & $-0.00184 \pm 0.0343$ & $102.89 \%$ \\
\hline
\end{tabular}

